\title{
A LONGITUDINAL STUDY OF DYNAMIC RISK, PROTECTIVE FACTORS, AND CRIMINAL RECIDIVISM: CHANGE OVER TIME AND THE IMPACT OF ASSESSMENT TIMING
}

\author{
by \\ Laura Hanby \\ A thesis submitted to \\ the Faculty of Graduate and Postdoctoral Affairs \\ in partial fulfillment of the requirements for the degree of \\ Doctor of Philosophy \\ in \\ Psychology \\ Carleton University \\ Ottawa, Canada \\ (C) 2013 Laura Hanby
}




\begin{abstract}
Risk assessment and risk management are central to most decisions made about offenders, particularly when considering community release after a period of incarceration. Although the field of risk assessment has progressed considerably, there remain limitations within current practices. The present research uses a variety of sophisticated statistical techniques to examine the systematic assessment and reassessments of risk in a large sample $(N=3498)$ of New Zealand parolees. The validity of the Dynamic Risk Assessment for Offender Re-entry (DRAOR), a measure of dynamic risk and protective factors, was assessed across time in all offenders released on parole. The measure demonstrated acceptable psychometric properties, although future research should seek to refine the subscales as suggested by its factor structure. Beyond validating the DRAOR, this study showed that reconvictions and criminal reconvictions during a two-year follow-up period can be accurately predicted from dynamic risk factors and protective factors (as measured by the DRAOR). Stable and acute dynamic risk scores decreased over time while protective factor scores increased, suggesting that the DRAOR is sensitive to change. Recidivists differed from non-recidivists in stable dynamic risk and protective factors in the month prior to follow-up end and in acute dynamic risk in the second month prior to follow-up end. Reconvictions were accurately predicted from monthly average Stable Risk beginning at parole start and continuing for 12 months of assessments, while Protective Factors were predictive for the first 4 months only. These results indicate that the DRAOR has promise as a valid tool for risk assessment and risk management. The findings of this study highlight the mechanisms
\end{abstract}


by which risk changes over time and provides support for a transitional model of offender re-entry focusing on dynamic risk and protective factors. 


\section{Acknowledgements}

With the deepest gratitude I thank my supervisor, Dr. Ralph Serin. I truly appreciate the endless opportunities, support, and advice he has given me as my mentor. I am also very grateful to my committee members, Dr. Shelley Brown and Dr. Craig Bennell, for their insights and constructive feedback as I developed my research. A special thank you to Dr. Bill Marshall, my external examiner, and Dr. Katharine Kelly, my internal examiner, for contributing their knowledge and enthusiasm.

This project would not have been possible without the cooperation of the New Zealand Department of Corrections, and the invaluable assistance of Dr. Nick Wilson in particular. A rich dataset with over 97,000 assessments on almost 3,500 offenders is a researcher's dream come true! A number of colleagues helped me out along the way, and without a doubt, raised the calibre of my work. Thank you to Caleb Lloyd and Leslie Helmus for their incredible expertise as I devised my statistical analyses, and to Rebecca Mugford, Kelly Babchishin, and Angela Smeth for taking the time out of their busy schedules to provide feedback on my dissertation. I am also thankful to Dr. Larry Motiuk and Ben Vuong for their support during my doctoral studies and to the Social Sciences and Humanities Research Council for providing financial assistance.

Writing a thesis while juggling life with a newborn baby is a challenge, but my beautiful son, Eli, provided me the inspiration to see this project through. May he learn from my example and see that anything is possible. A special thanks to my partner, Geoff, for supporting my dream and taking on extra diapers, bath times, and early mornings to make my dream possible. Last but not least, I appreciate the unwavering encouragement from my family, particularly Mam, Dad, Dean, Kristina, and Jack. 


\section{Table of Contents}

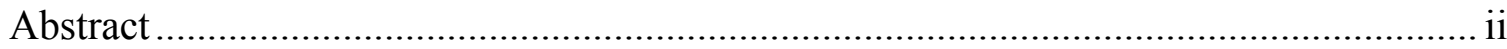

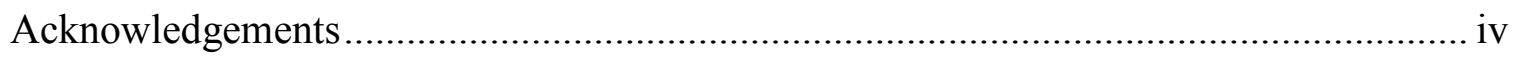

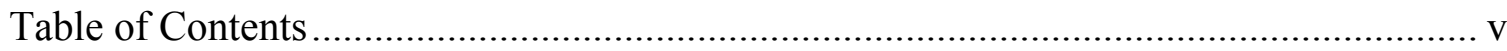

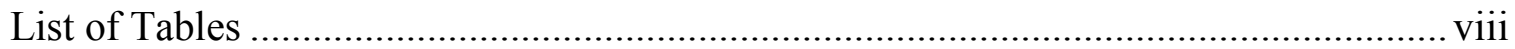

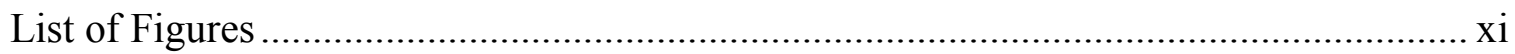

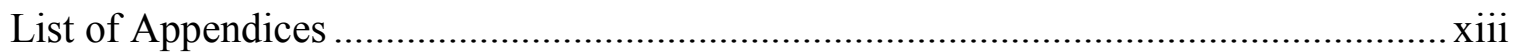

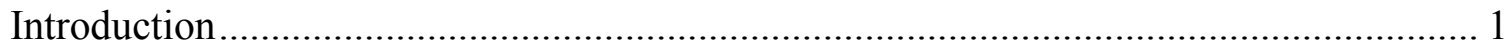

Evolution of Risk Assessment .......................................................................... 4

Limitations of Current Risk Assessment Measures .................................................. 7

Unique Contribution of the DRAOR .................................................................. 15

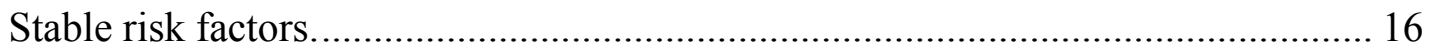

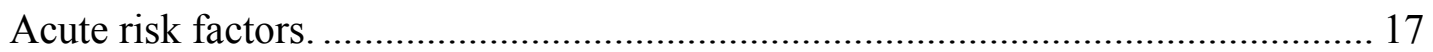

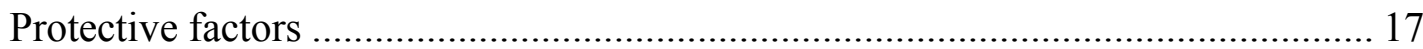

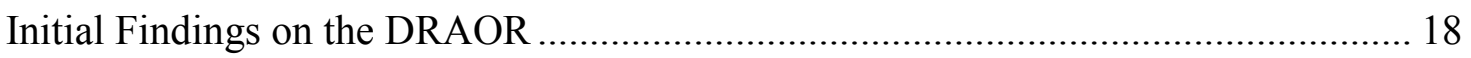

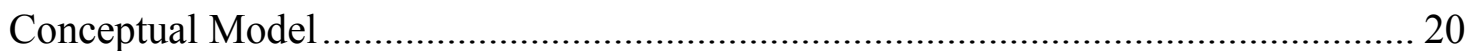

Improving risk management with dynamic factors .......................................... 25

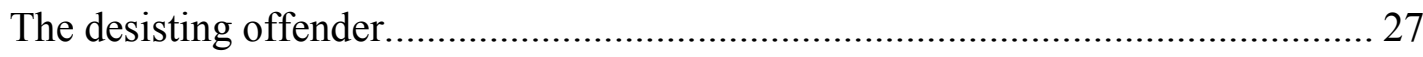

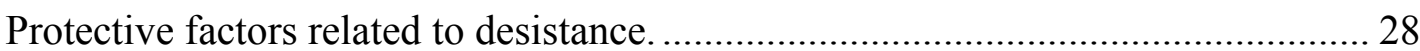

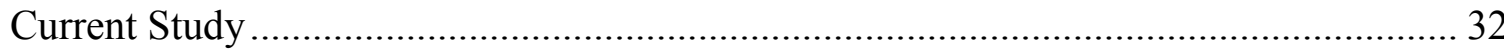

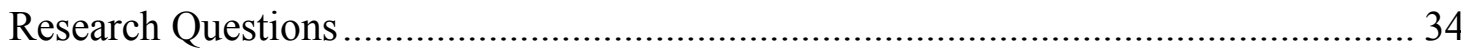

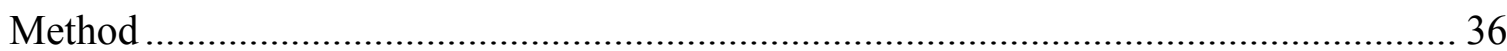

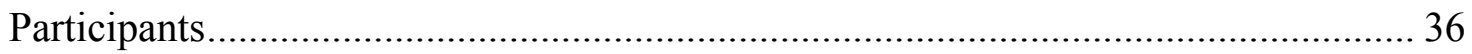

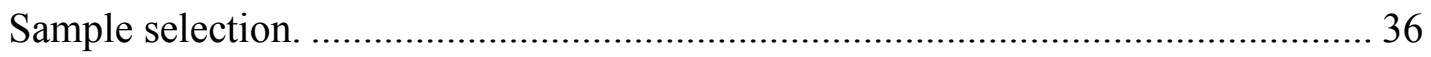

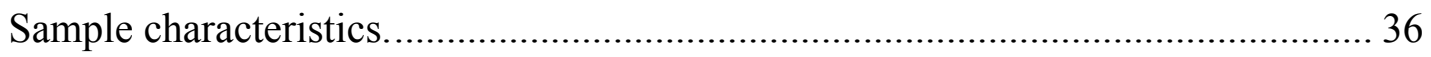

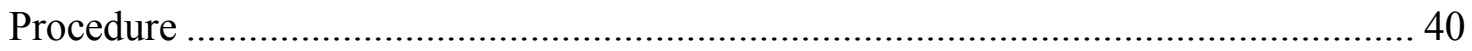

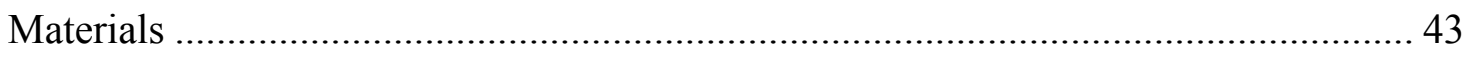

Dynamic Risk Assessment for Offender Re-entry.............................................. 43

Stable risk factors............................................................................. 44

Acute risk factors ............................................................................ 47

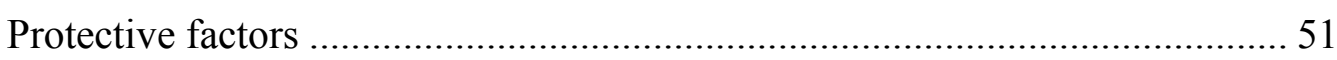

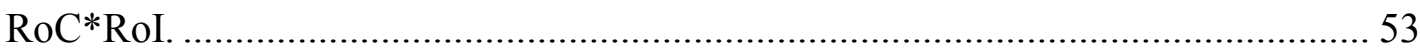

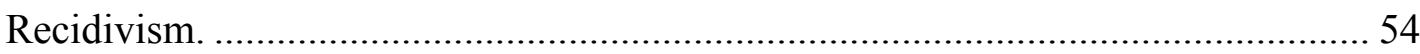

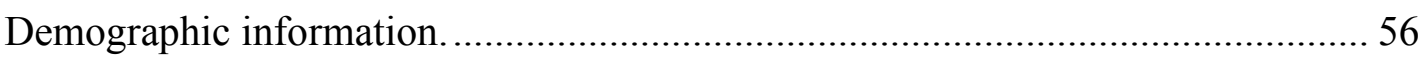




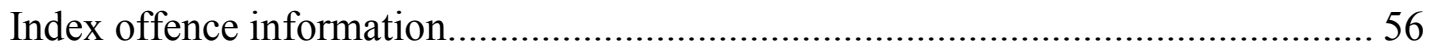

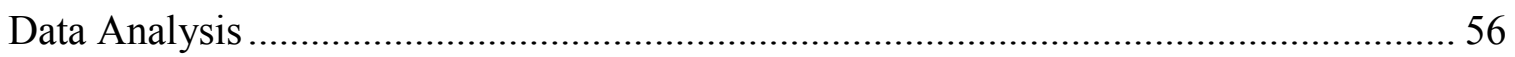

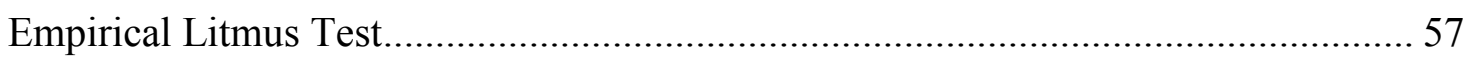

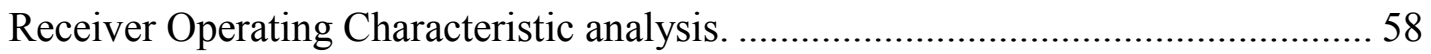

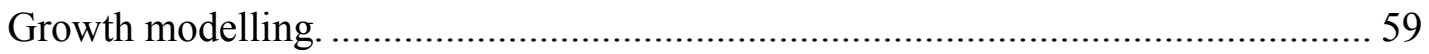

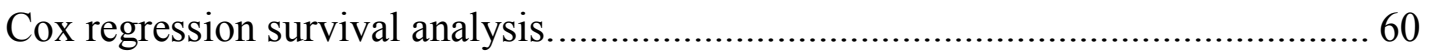

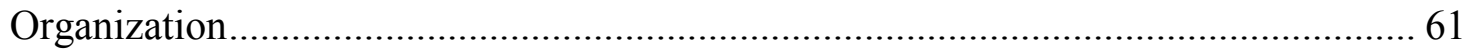

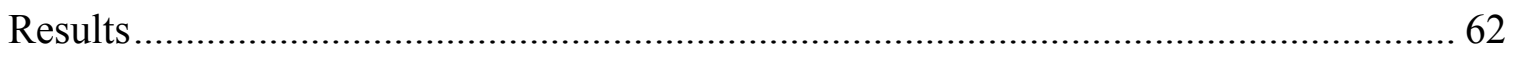

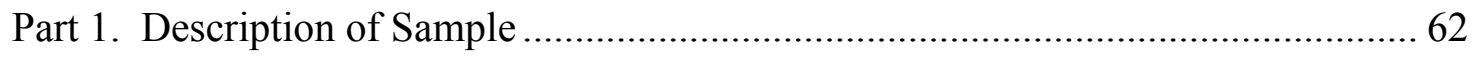

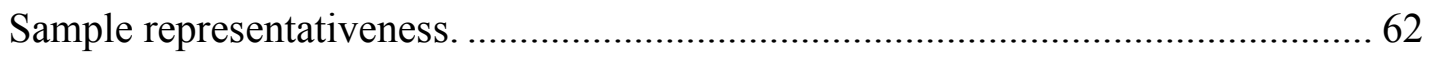

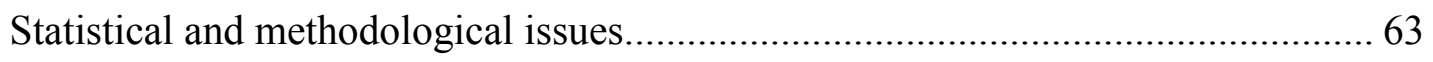

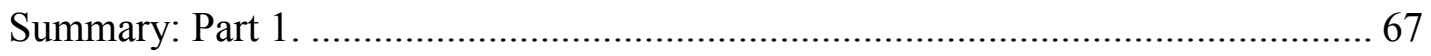

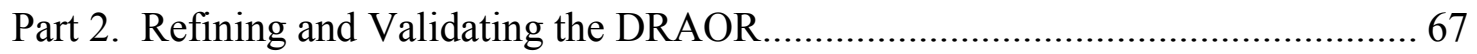

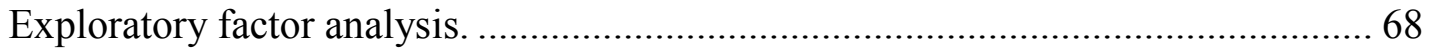

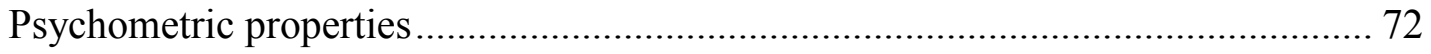

Confirmatory factor analysis........................................................................... 74

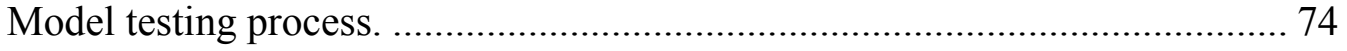

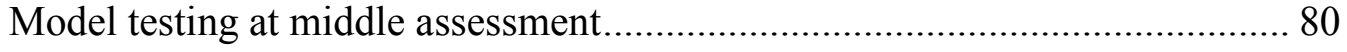

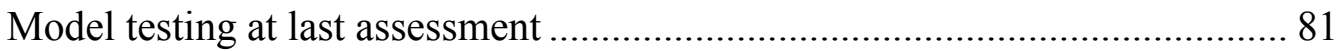

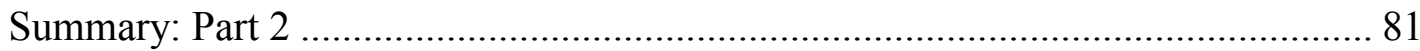

Part 3. Differentiation of Recidivists and Non-Recidivists ....................................... 83

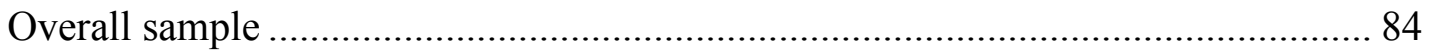

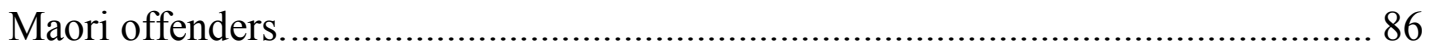

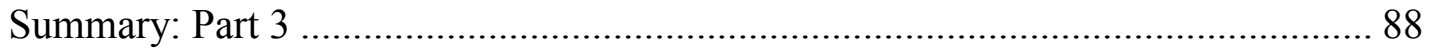

Part 4. Prediction of Recidivism from Dynamic and Static Measures ....................... 89

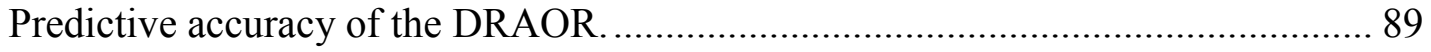

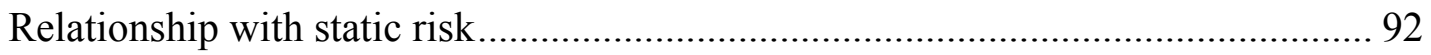

Summary: Part 4 .............................................................................................. 97

Part 5. Change in Dynamic Risk Over a Period of Assessment and Reassessment .... 98

Model building process ..................................................................................... 98

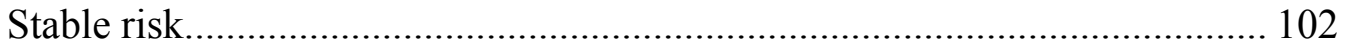

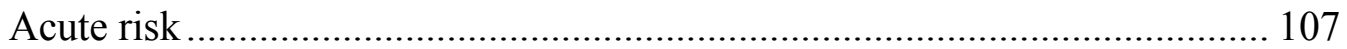

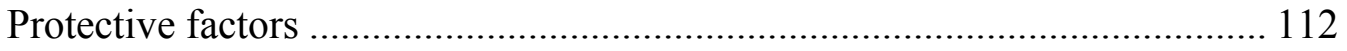

Recidivists versus non-recidivists hypothesis testing............................................ 117 


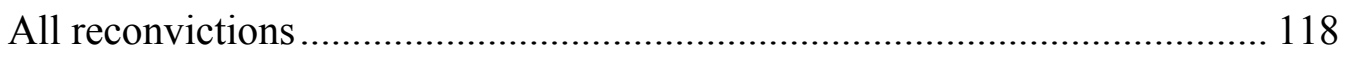

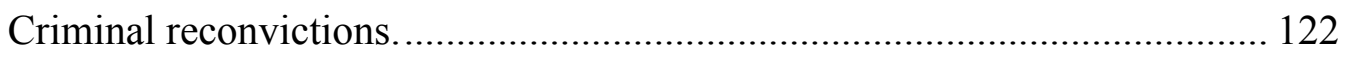

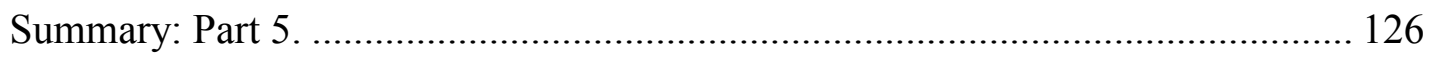

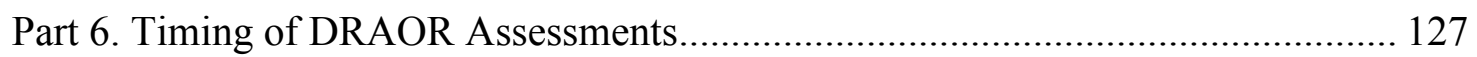

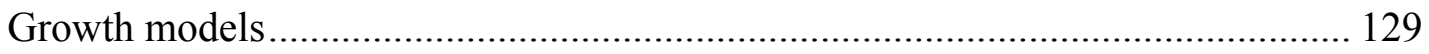

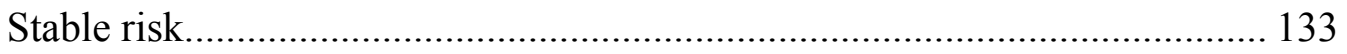

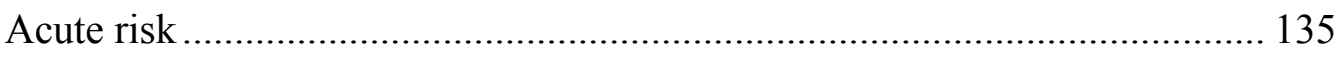

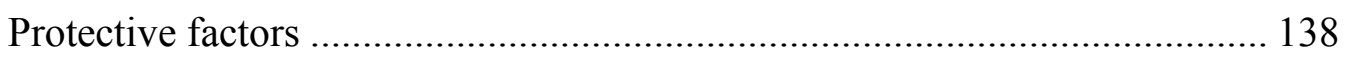

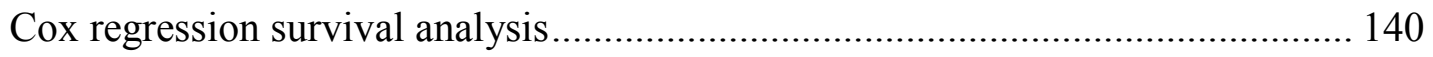

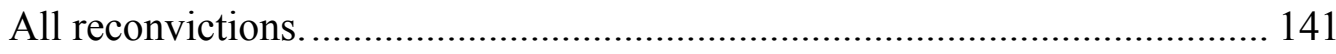

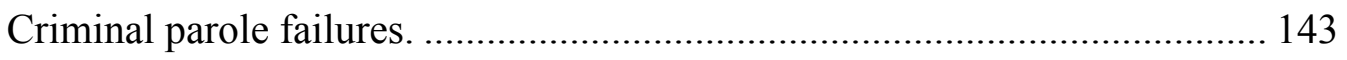

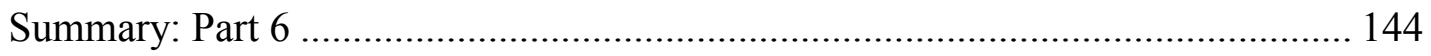

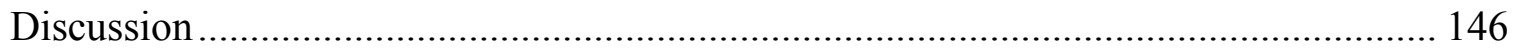

Summary of Findings.............................................................................. 146

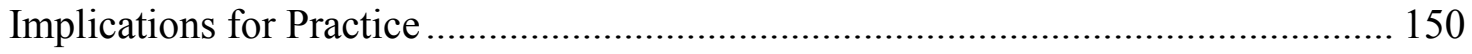

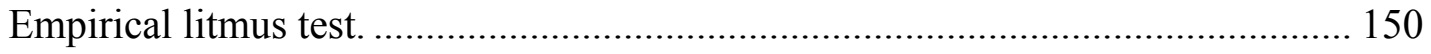

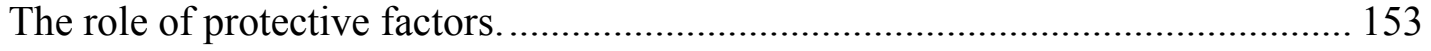

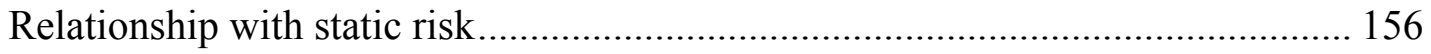

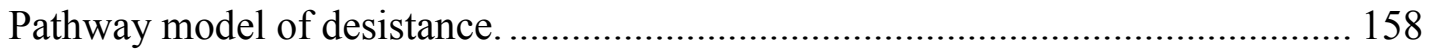

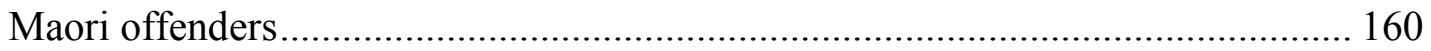

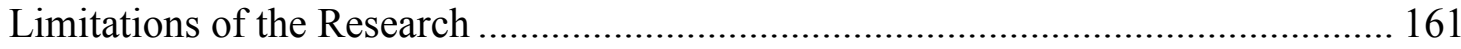

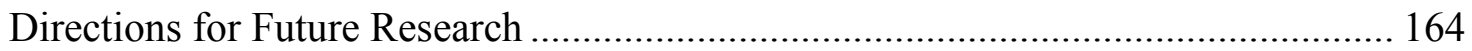

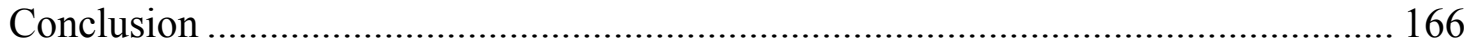

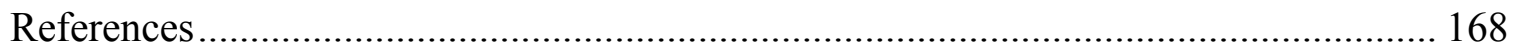




\section{List of Tables}

Table 1. Sample Demographics 37

Table 2. Most Serious Offence Characteristics Related to Conviction ............................ 38

Table 3. Most Serious Offence Characteristics Related to Reconviction ......................... 39

Table 4. Frequency of Contact between Probation Officers and Parolees $(N=3498) \ldots 41$

Table 5. Sample Representativeness: Study Sample $(N=3498)$ vs. 2008/2009 Offender Population $(N=4514)$

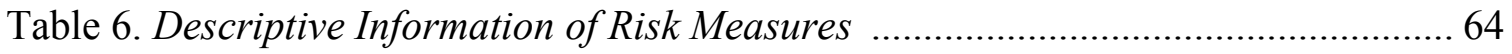

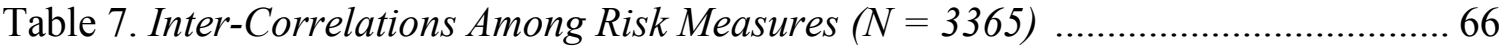

Table 8. Internal Consistency and Descriptive Statistics for Model \#1 DRAOR Subscales

Table 9. Internal Consistency and Descriptive Statistics for Model \#2 DRAOR Factors 73

Table 10. Internal Consistency and Descriptive Statistics for Model \#3 DRAOR Factors

Table 11. Goodness-of-Fit Indices for Three Models using the Middle DRAOR

Assessment

Table 12. Goodness-of-Fit Indices for Three Models Using the Last DRAOR Assessment

Table 13. Comparison of Recidivists versus Non-recidivists for Overall Sample 85

Table 14. Comparison of Criminal Recidivists versus Non-recidivists for Overall Sample 86

Table 15. Comparison of Recidivists versus Non-recidivists for Maori Offenders 87 
Table 16. Comparison of Criminal Recidivists versus Non-recidivists for Maori Offenders 88

Table 17. Predictive Accuracy of the DRAOR: Overall Sample $(N=3372)$................... 91

Table 18. Predictive Accuracy of the DRAOR: Maori Offenders $(N=1755)$................. 91

Table 19. Incremental Validity of the DRAOR and RoC*RoI for Predicting All

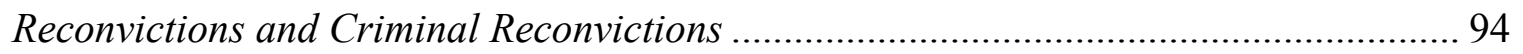

Table 20. Predictive Accuracy of the RoC*RoI $(N=3372)$ and Combined Static and

Dynamic Models $(N=3365):$ Overall Sample ........................................................ 95

Table 21. Comparison of Static and Dynamic Models: Overall Sample $(N=3365) \ldots . . . .96$

Table 22. Predictive Accuracy of the RoC*RoI $(N=1755)$ and Combined Static and

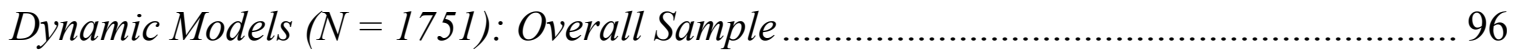

Table 23. Comparison of Static and Dynamic Models: Maori Offenders $(N=1751) \ldots . .97$

Table 24. Taxonomy of Multilevel Models for Change Fitted to Stable Risk Data ........ 105

Table 25. Results of Fitting a Taxonomy of Multilevel Models for Change for Stable Risk

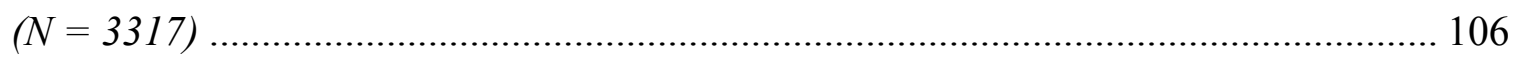

Table 26. Taxonomy of Multilevel Models for Change Fitted to Acute Risk Data ........ 110

Table 27. Results of Fitting a Taxonomy of Multilevel Models for Change for Acute Risk

$(N=3317)$

Table 28. Taxonomy of Multilevel Models for Change Fitted to Protective Factors Data 115

Table 29. Results of Fitting a Taxonomy of Multilevel Models for Change for Protective

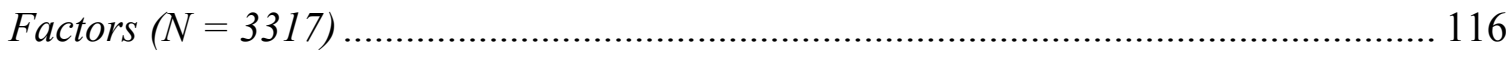

Table 30. Results of Hypothesis Testing for All Reconvictions $(N=3317)$.................. 119 
Table 31. Results of Hypothesis Testing for Criminal Reconvictions $(N=3317) \ldots \ldots . . . .123$

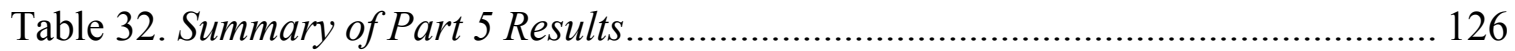

Table 33. Results of Multilevel Modelling of Stable Risk Using Monthly Time Variables

$(N=3240)$

Table 34. Results of Multilevel Modelling of Acute Risk Using Monthly Time Variables (N $=3240)$

Table 35. Results of Multilevel Modelling of Protective Factors Using Monthly Time Variables $(N=3240)$

Table 36. Cox Regression Analysis of DRAOR Variables on Survival Time of Offenders for All Reconvictions 142

Table 37. Cox Regression Analysis of DRAOR Variables on Survival Time of Offenders for Criminal Reconvictions 145 


\section{List of Figures}

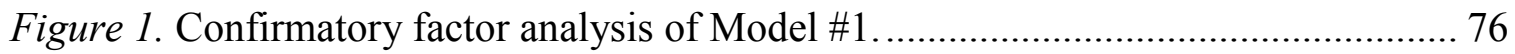

Figure 2. Confirmatory factor analysis of Model \#2 .............................................. 77

Figure 3. Confirmatory factor analysis of Model \#3 .............................................. 78

Figure 4. Graph of prototypical change trajectories using the Stable Risk models. ...... 107

Figure 5. Graph of prototypical change trajectories using the Acute Risk models....... 112

Figure 6. Graph of prototypical change trajectories using the Protective Factor models.

Figure 7. Graph of prototypical change trajectories in Stable Risk using all reconvictions.

Figure 8. Graph of prototypical change trajectories in Acute Risk using all reconvictions.

Figure 9. Graph of prototypical change trajectories in Protective Factors using all reconvictions

Figure 10. Graph of prototypical change trajectories in Stable Risk using criminal reconvictions

Figure 11. Graph of prototypical change trajectories in Acute Risk using criminal reconvictions 125

Figure 12. Graph of prototypical change trajectories in Protective Factors using criminal reconvictions. 125

Figure 13. Graph of Stable Risk Unconditional Growth Model using monthly variables.

Figure 14. Graph of Stable Risk All Reconvictions Model using monthly variables. ... 134 
Figure 15. Graph of Stable Risk Criminal Reconvictions Model using monthly variables.

Figure 16. Graph of Acute Risk Unconditional Growth Model using monthly variables.

Figure 17. Graph of Acute Risk All Reconvictions Model using monthly variables.... 137

Figure 18. Graph of Acute Risk Criminal Reconvictions Model using monthly variables.

Figure 19. Graph of Protective Factors Unconditional Growth Model using monthly variables

Figure 20. Graph of Protective Factors All Reconvictions Model using monthly variables.

Figure 21. Graph of Protective Factors Criminal Reconvictions Model using monthly variables.

Figure 22. Effects sizes for 12 models based on monthly average DRAOR subscales. 143 


\section{List of Appendices}

Appendix A. Risk of re-Conviction X Risk of re-Imprisonment Model (RoC*RoI) ..... 193

Appendix B. Inter-Correlations between DRAOR Items ................................................. 194

Appendix C. Exploratory Factor Analysis ................................................................. 195

Appendix D. Reliability Analyses of Model \#1 (Original DRAOR)............................. 199

Appendix E. Reliability Analyses of Model \#2 of the DRAOR ....................................... 200

Appendix F. Reliability Analyses of Model \#3 of the DRAOR ...................................... 202

Appendix G. Base Rates of Reconvictions by DRAOR Total Score ............................... 204

Appendix H. Multilevel Models for Change for Reconviction and Criminal Reconviction

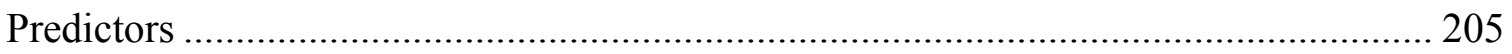

Appendix I. Multilevel Models for Change for Timing of DRAOR Assessments......... 207 
A Longitudinal Study of Dynamic Risk, Protective Factors, and Criminal Recidivism:

Change over Time and the Impact of Assessment Timing

Compared to two decades ago, more offenders are released from prison on some form of community supervision (Serin, Lloyd, \& Hanby, 2010). Risk assessment is central to most management decisions made about offenders, including who should be released, what restrictions are appropriate and what rehabilitative efforts would be effective (Andrews \& Bonta, 2010a, 2010b). Estimating an offender's likelihood of offending once released into the community is one of the most important functions of a correctional agency, and accordingly, has been extensively investigated (Hanson, 2009). In addition to risk assessment, it is important to be able to detect changes in an offender's level of risk such that potential failure can be prevented and managed through appropriate treatment and supervision. By carefully monitoring factors related to reoffending through risk management, it is possible to provide the necessary interventions before an offender reaches the point of no return (Hanson, 2009).

Risk assessment has evolved considerably, beginning with an approach of unstructured clinical judgment which offered much professional discretion and subjectivity. It then shifted to a purely actuarial approach with a focus on historical, static risk factors. Yet while on release, offenders are subject to events and experiences that may produce shifts in their likelihood of recidivating (Andrews, 1989). With this in mind, later methods of risk assessment have expanded beyond static factors to include changeable, dynamic risk factors while striving for a balance between professional discretion and these empirically informed risk factors. 
Despite the progression of the field of risk assessment, there are still limitations to current practice and research. Risk assessment has entered a fourth generation in which offender risk and needs are still the central focus, along with an attempt to explicitly link identified needs with supervision and treatment services (Andrews, Bonta, \& Wormith, 2006). Given risk changes, in order to accurately assess risk and manage it accordingly, instruments should incorporate both static and dynamic risk factors (Andrews \& Bonta, 2010a). Moreover, protective factors, or circumstances that insulate an offender from engaging in criminal behaviour when faced with an at-risk situation, would also seem relevant, at least at a conceptual level. Although both static and dynamic risk factors are statistically related to offending behaviour, dynamic factors can potentially change and result in increased or decreased recidivism risk. Risk assessment tools that measure dynamic factors are sensitive to change and are capable of measuring factors that are changeable and related to criminal behaviour (Brown, 2002). In addition, very few risk assessment measures take into account offender strengths, or protective factors. These internal or external variables may mitigate an offender's likelihood of engaging in crime, and accordingly are an important component of risk assessment.

The Dynamic Risk Assessment for Offender Re-entry (DRAOR) was developed as a user-friendly risk assessment instrument with the advantages to risk management associated with combining both dynamic risk factors and protective factors (Serin, 2007; Serin, Mailloux, \& Wilson, 2010). The DRAOR was developed to assist parole and probation officers to systematically review changes in an offender's community situation, thereby responding with appropriate supervision strategies based on a rationally-derived model of risk. It represents an integration of several theoretical works, reflected 
primarily in a hybrid model of offender re-entry (Serin, Lloyd et al., 2010). This model presents a life-course perspective that incorporates risk factors for crime acquisition and desistance correlates. Given that the DRAOR distinguishes between risk and protective factors, it may also improve our understanding of the process of and mechanisms underlying crime desistance. The transition to crime desistance is gradual and complex, believed to involve both internal and external change factors that will influence an offender's likelihood of successful re-entry (Serin \& Lloyd, 2009; Serin, Lloyd et al., 2010).

The DRAOR has been piloted in locations in New Zealand (Tamatea \& Wilson, 2009) and the United States (Serin \& Prell, 2012) with promising results. Based on the pilot work in the United States, the DRAOR is a moderate predictor of general recidivism $(\mathrm{AUC}=.66 ; 95 \% \mathrm{CI}=[.62, .69])$. In addition, the total Stable Risk scores $(\mathrm{AUC}=.60$ $95 \% \mathrm{CI}=[.56, .64])$, the total Acute Risk scores $(\mathrm{AUC}=.65 ; 95 \% \mathrm{CI}=[.61, .69])$, and the total Protective Factor scores $(\mathrm{AUC}=.67 ; 95 \% \mathrm{CI}=[.63, .70])$ have all demonstrated moderate predictive accuracy for technical violations and general recidivism. The very small pilot $(N=59)$ in New Zealand indicated that DRAOR scores changed across assessments, that Stable Risk, Acute Risk, and Protective Factor scores were moderately correlated $(r=.41-.52)$, that recidivists had significantly lower initial and final Protective scores, and that the Protective score added incremental validity to a static risk score. Feedback from probation officers was positive, regarding the DRAOR as an understandable assessment approach in a user-friendly format. This led to the expansion of the DRAOR in New Zealand, paving the way for the current study. 
The current research endeavour is a longitudinal study of a large sample of parolees $(N=3498)$ in New Zealand who were administered the DRAOR by trained probation officers. The purpose of this study was to validate and refine the DRAOR to determine if and how it can be improved. In addition, this study included an examination of whether recidivism can be predicted from dynamic within-offender variables. This line of questioning also addressed whether the assessment of dynamic risk improves predictive validity above and beyond static risk. Finally, a more theoretical examination of change in dynamic risk was investigated, specifically whether or not dynamic factors actually change over a period of systematic assessment and reassessment, and at what point the DRAOR becomes predictive of parole failures. These research goals will help advance our understanding of risk assessment and protective factors, as well as the crime desistance process.

\section{Evolution of Risk Assessment}

Offender risk assessment has evolved rapidly beginning with a subjective approach of unstructured clinical judgment. This first generation of risk assessment involved unstructured professional opinions and judgments based on intuition, wisdom, and experience (Bonta, 1996). The inherent weakness of such an unstructured approach is a lack of consistency and agreement between different clinicians, resulting in low interrater reliability (Webster, Douglas, Eaves, \& Hart, 1997a). It dominated corrections for several decades and despite its disadvantages, remains preferred by many correctional decision makers (Boothby \& Clements, 2000).

After affording much discretion to clinicians, the second generation was marked by a shift to a more objective approach to risk assessment with the use of actuarial tools 
(Bonta, 1996). These instruments involve statistically weighting and combining a set of factors that have been found to be correlated with the risk of recidivism. Actuarial methods mainly consist of static factors, which are historical and unchangeable. It is well accepted in the literature and confirmed in a number of meta-analyses that in most cases actuarial risk assessment tools outperform clinical judgment (Bonta, Law, \& Hanson, 1998; Grove \& Meehl, 1996; Mossman, 1994). However, actuarial tools have been criticized for their limited clinical utility and limited value for understanding the etiology of criminal behaviour and violence (Dvoskin \& Heilbrun, 2001; Grubin \& Wingate, 1996; Litwack, 2001). Actuarial tools based on static factors also cannot measure changes in risk and as a result, provide little guidance for risk management beyond a baseline decision (i.e., greater frequency of contact for higher risk cases). Static factors do not indicate when an offence is likely to occur or if interventions have produced enough change to decrease the probability of reoffending.

The third generation of risk assessment incorporates both static risk factors and dynamic risk factors, which are changeable, but durable (i.e., stable) or transient (i.e., acute; Bonta, 1996). The underlying assumption is that risk is not entirely stable and can change as a result of factors such as treatment quality and quantity, context, development factors, and protective factors (Vincent, Chapman, \& Cook, 2011). Often referred to as risk-needs instruments, these assessments provide information regarding the dynamic factors, or criminogenic needs, that should be treated to reduce the offender's risk. The actuarial nature of second and third generation tools in terms of attaching specific probabilities based on a risk score have also been questioned in the fields of forensic mental health (Grisso \& Applebaum, 1992) and violence (Hart \& Cooke, 2013). 
Nonetheless, risk-needs assessments continue to be the most widely accepted and commonly used method among forensic psychologists (Archer, Buffington-Vollum, Stredny, \& Handel, 2006).

A fourth generation of risk assessments have recently begun to make their way into practice (Andrews et al., 2006). Fourth generation tools consist of an integrated case plan system that is directly based on the results of the assessment and helps staff target criminogenic needs identified as moderate to high risk (Latessa \& Lovins, 2010). The Level of Service/Case Management Inventory (LS/CMI; Andrews, Bonta, \& Wormith, 2004) is an example of this approach, combining the assessment of risk and need factors with a case management function to aid in treatment planning and the management of offenders. Similar to the third generation, this approach to risk assessment includes riskneeds instruments but also goes beyond by assessing a broader range of risk factors as well as responsivity factors. Treatment responsivity refers to dynamic, client-based factors which influence the potential for offender change (Kennedy \& Serin, 1999). It indicates the extent to which offenders are able to absorb program content based on their cognitive ability, learning style, and values (Ward, Day, Howells, \& Birgden, 2004). This generation of risk assessment attempts to explicitly link identified needs with supervision and treatment services (Bonta \& Wormith, 2007). The current generation has been criticized by Baird (2009) as problematic, arguing that risk should be measured by static factors and criminogenic needs should be assessed separately. On the other hand, fourth generation tools have been described as practical and just as predictive as tools measuring only risk (Bonta \& Wormith, 2007). 
Although the generations of risk assessment categorized by Bonta (1996) and Andrews and colleagues (2006) are widely recognized, they do not accurately capture all risk tools (Hanson \& Morton-Bourgon, 2009). For instance, Structured Professional Judgment (SPJ) is an approach in which the clinician evaluates risk using a structured assessment of a set of empirically-based risk factors (Borum, 1996). Individual risk assessments of the specific probability of future offending are not made. Instead, the guidelines help evaluators determine potential types of reoffending and victims, under what circumstances reoffending is likely, and provide recommendations on how to prevent recidivism (Hart \& Cooke, 2013). There is less of a consensus on the accuracy of SPJ in relation to actuarial tools. Some research indicates that SPJ decisions about risk may have higher predictive validity over simple score-based decisions (Douglas, Yeoman, \& Boer, 2005), but the findings are inconsistent as to which is superior (Hanson, 2009). These tools do not fit into the actuarial approach of second and third generations, but they provide more structure and assess a broader range of factors than first generation assessments. Of note, Hanson (1998) developed a classification system for risk assessment instruments into SPJ, pure actuarial, and clinically-adjusted actuarial. This recognizes that the debate about unstructured and structured risk assessment goes beyond the predictive accuracy of these approaches to the purpose of risk assessment and the role of professional judgment (Hanson \& Morton-Bourgon, 2009).

\section{Limitations of Current Risk Assessment Measures}

The principal focus of risk assessment for the past three decades has been on static factors to determine inter-individual differences, although increasingly there is interest in the contribution of dynamic risk factors (Hanson \& Harris, 2001). Static 
factors are useful because they are predictive of future offending, but are limited in that they do not provide information on an offender's current functioning, nor situations that may increase their risk of offending. Static factors also cannot be used to assess changes in a person, limiting their utility to providers of treatment or management services (Wong, Olver, \& Stockdale, 2009). Although both types of risk factors are statistically related to offending behaviour, dynamic factors can potentially change and result in increased or decreased recidivism risk. In contrast, static risk factors are predictive based on their presence or absence, but cannot reduce risk over time. Therefore, in order to detect shifts in an offender's likelihood of recidivism, dynamic risk factors must be assessed (Andrews, 1989). Including dynamic factors in risk assessment helps guide the delivery of rehabilitation services and allows for the measurement of change (Simourd, 2004).

Douglas and Skeem's (2005) distinction between risk status and risk state serves as a useful framework to guide a discussion regarding the relative contribution of types of factors in the assessment of risk. They note that the majority of past research has focused on risk status, which is heavily reliant on static risk factors to assess an individual's level of risk relative to others. Risk assessment measures focusing on static risk are not designed to detect changes in risk necessary for managing ongoing offending. Focusing on risk status also fails to identify meaningful treatment targets to guide intervention. Douglas and Skeem recommend focusing on risk state, which acknowledges dynamic risk factors and measures the offender's propensity to commit an offence at a given time. This distinction between static and dynamic factors relates to whether or not a measurement of the variable is likely to fluctuate over time. Risk state is changeable over 
time and in response to intervention, and increases or decreases in risk state should be accompanied by changes in risk management. It helps determine not only who will recidivate (i.e., risk status), but also when an offender may recidivate (i.e., risk state). Having information on both the 'who' and the 'when' can therefore help determine how to reduce the likelihood of reoffending.

In addition to dynamic factors, evidence has been found for the moderating influence of strengths on risk factors. For example, in a sample of young offenders, high scores on a scale of strengths (e.g., positive relationships with staff, leisure activities) appeared to act as a protective factor even if an individual had a high score on a scale of concerns (e.g. impulsive behaviours, pro-criminal peers; Griffin, Beech, Print, Bradshaw, \& Quayle, 2008). However, most risk assessment and risk management practices fail to take into account offender strengths, or protective factors, the internal and external variables that may mitigate an individual's offending behaviour. Rutter (1985) describes protective factors as ones that modify, ameliorate, or alter a person's response to a hazard that predisposes a maladaptive outcome. Focusing solely on risk factors to the neglect of protective factors has been argued to promote a biased and one-sided examination of human behaviour (Rogers, 2000). Risk assessment tools that incorporate only dynamic and static risk factors provide a vital foundation for the evaluation of risk, but do not offer adequate guidance for treatment (Nicholls, Brink, Desmarais, Webster, \& Martin, 2006) or crime desistance. Dynamic risk factors form the focus of intervention efforts and can provide a measure of change in risk level by conducting reassessments (Andrews \& Bonta, 2010b). Protective factors can moderate the effects of risk factors on behaviour when risk is high and therefore reduce the likelihood of reoffending (Werner \& Smith, 
1992). Clinicians have reported that protective factors are as important to clinical practice as risk factors (Stübner, Groß, \& Nedopil, 2006).

Protective factors are highlighted in relation to a pathway model of offending and desistance from crime (Polaschek, 2003). Desistance from crime is recognized as a process in which internal and external variables align in such a way that an offender with a history of crime ceases all criminal activity (Serin \& Lloyd, 2009). A pathway model recognizes that there are variable models of offending, and therefore varying mechanisms and processes potentially responsible for offending and desistance (Tamatea \& Wilson, 2009). An improved understanding of protective factors and their contribution to offence and desistance pathways may enhance our understanding of offender management. This has the potential to advance the field beyond existing measures that only consider static and dynamic risk. The importance of the inclusion of protective factors and risk management strategies in risk assessment has been highlighted by others in the field (e.g., Hanson, 2009).

There are few risk assessment tools that include protective factors, but most of the existing tools were developed for specific populations. Protective factors have particularly been a focus in mental health populations as well as youth offenders, but not in adult criminal behaviour, with the exception of the Inventory of Offender Risk, Needs, and Strengths (IORNS; Miller, 2006). The Short Term Assessment of Risk and Treatability (START; Webster, Martin, Brink, Nicholls, \& Middleton, 2004), Historical, Clinical, Risk Management-20 (HCR-20; Webster, Douglas, Eaves, \& Hart, 1997b), and Structured Assessment of Protective Factors for Violence Risk (SAPROF; De Vogel, De Ruiter, De Bouman, \& de Vries Robbé, 2007) are all risk assessment tools that have been 
developed for mentally disordered offenders. Lastly, the Structured Assessment of Violence Risk in Youth (SAVRY; Borum, Bartel, \& Forth, 2006) represents an important step forward in risk assessment and management with young offender populations. Each of these tools will be briefly discussed in turn.

The START (Webster et al., 2004) is a structured instrument for judgment of dynamic risk and treatment factors in mentally and personality disordered clients. Its aim is to assess their short-term risk of violence to others as well as risk of suicide, self-harm, self-neglect, substance abuse, unauthorized leave, and victimization. This assessment scheme consists of 20 positive and negative items which are rated as vulnerability or strength factors, with the option of designating certain items as being "critical" risks or "key" strengths. In a study of psychiatric patients in a high secure unit in Norway over 20 months, Strength scores demonstrated good predictive accuracy $(\mathrm{AUC}=.75)$ in predicting severe violence against staff (Nonstad et al., 2010). Early findings also suggest the START is associated with aggressive behaviour against others (Nicholls et al., 2006). However, a recent study examining the predictive validity of the START found that a limited set of START items combined was significantly better at predicting a range of challenging behaviours (i.e., aggression, self-harm, and substance abuse) than the original Strength and Vulnerability scales (Braithwaite, Charette, Crocker, \& Reyes, 2010). Further prospective research is necessary to establish the reliability and validity of the START, given that much of the research to date has consisted of file reviews by research assistants.

The HCR-20 (Webster et al., 1997b) is a well known SPJ instrument consisting of 10 historical (static) variables, 5 clinical variables, and 5 risk management variables. The 
clinical items measure current symptomatology and psychosocial adjustment, while the risk management items address release and treatment plans, necessary services, and support. The risk management items contain elements of protective factors by focusing on factors that may aggravate or mitigate risk and influence how offenders will adjust to future circumstances and living contexts (e.g., exposure to destabilizers, lack of personal support). The HCR-20 has been shown to predict general and violence recidivism in forensic clients (Douglas et al., 2005). However, it measures few protective factors and does so less directly than other risk assessment tools discussed here.

The SAPROF (De Vogel et al., 2007) was developed to complement other SPJ tools (e.g., HCR-20) to create a more balanced assessment of risk for future violence. It is the only instrument focusing exclusively on protective factors, consisting of 17 items that are categorized as internal factors (e.g., self control), motivational factors (e.g., life goals), and external factors (e.g., social network). Findings suggest the SAPROF has good inter-rater reliability and predictive validity for violent recidivism after treatment, although it is still in its initial stages of development (De Vogel, De Ruiter, De Bouman, \& de Vries Robbé, 2009). These results hold true for both violent offenders and sex offenders (De Vries Robbé \& De Vogel, 2010). Overall the predictive accuracy of the SAPROF in combination with the HCR-20 $(\mathrm{AUC}=.72)$ has been found to be significantly higher than the HCR-20 alone $(\mathrm{AUC}=.68)$ in male violent and sexual forensic psychiatric patients during a three year follow-up (De Vries Robbé, De Vogel, \& De Spa, 2011; De Vries Robbé, De Vogel, Koster, \& Bogaerts, in preparation). The SAPROF appears to be the most tested and published of the risk assessment tools 
containing protective factors. Its practical utility, given that it is a separate measure to be used in conjunction with a risk assessment tool, remains to be determined.

The SAVRY (Borum et al., 2006) was developed to assess risk for violence in adolescents aged 12 to 18 years. It consists of protective factors as well as static and dynamic risk factors divided into historical, social/contextual, and individual/clinical items. The protective factors include community involvement, social support, and personality traits. The SAVRY has been demonstrated to predict disruptive behaviour during residential treatment including violence against persons and objects, verbal threats, and violations of rules (Lodewijks, Doreleijers, de Ruiter, \& Borum, 2008). In addition to institutional violence, it has been found to be predictive of subsequent general and violent offending in forensic and young offender populations (Borum, Lodewijks, Bartel, \& Forth, 2009; Vincent et al., 2011). The SAVRY represents the newest direction in terms of risk assessment with young offender populations, but further research is warranted to fully validate the instrument.

Lastly, the IORNS (Miller, 2006) is a self-report measure that assesses static, dynamic, and protective factors in adult offenders to predict aggression and recidivism. It consists of 130 true/false items related to recidivism, treatment need, and management. The protective factors include cognitive/behavioural regulation, anger regulation, and education and training. An overall risk is calculated by adding scores on the static and dynamic risk indices and subtracting the offender's score on the protective strength index. Higher overall risk scores correspond to higher treatment need and potential risk. Using a sample of 162 male offenders released from the New Jersey prison system, offenders who violated the halfway house rules scored significantly higher on overall risk 
and dynamic needs and lower on protective strengths than those who did not (Miller, 2006). However, these findings may be limited by the low base rate of violations and by the categorization of rule violators into an unbalanced design of two or more violations ( $n$ $=11)$ compared to offenders who did not violate or only violated once $(n=107)$. The IORNS demonstrated good convergent validity with other measures of static and dynamic risk such as the Level of Service Inventory-Revised (LSI-R; Andrews \& Bonta, 1995). The veracity of self-reports may limit the utility of the IORNS and only one published study could be located.

With the inclusion of protective factors, all of these risk assessment tools demonstrate advances in the field of risk assessment and risk management. The factors were selected for inclusion based on recommendations from clinical experts and/or reviews of the literature. Most of these instruments characterize the fourth generation of risk assessment in which responsivity factors are combined with risk-needs assessments in an attempt to create a more direct link to supervision and treatment services. Little research has been published on the predictive validity of these instruments. Pilot studies of these tools are beginning to be published, although more psychometric data is needed to fully validate these instruments. In addition, all of these risk assessment tools target specific populations, whether mentally disordered offenders or young offenders, with the exception of the IORNS. The DRAOR is intended for use with adult offenders in general and has the goal of predicting any type of recidivism, including violent offending. Lastly, a number of these tools lack a fully integrated theoretical model guiding the inclusion of risk and protective factors. 


\section{Unique Contribution of the DRAOR}

The Dynamic Risk Assessment for Offender Re-entry (DRAOR, Serin, Mailloux et al., 2010) is a SPJ instrument that considers Stable, Acute, and Protective factors. The three domains are empirically related to the risk of reoffending (Stable and Acute) and desistance from crime (Protective). The DRAOR was developed to assist parole and probation officers to systematically review changes in an offender's community situation and respond accordingly. An offender's circumstances may change after release, thus requiring both assessment and reassessment. The purpose of the DRAOR is both risk assessment and risk reduction. Therefore, the variables that comprise the DRAOR must not only demonstrate to be dynamic risk factors, but also that risk is reduced when the variables are successfully changed (Hanson \& Harris, 2000; Quinsey, Harris, Rice, \& Cormier, 2006; Skeem \& Monahan, 2011). These factors are expected to be variable in that they change within an offender, either spontaneously or by intervention. These variable factors can be considered causal if they can be shown to be manipulatable and when manipulated, change the risk of the outcome (Kraemer et al., 1997). Given that the DRAOR distinguishes between risk and protective factors, it may improve our understanding of the processes of and mechanisms underlying both recidivism and crime desistance.

The DRAOR is unique given a combination of elements, including its measurement of protective factors and a target population of all adult offenders as opposed to a specific population. It is distinct from other measures due to its systematic use by parole and probation officers with every contact with offenders, as well as its intended goal to predict technical violations, as well as general and violent recidivism. 
Using a structured approach to combine stable and acute risk factors with static risk has been found to improve predictive accuracy (Hanson, Harris, Scott, \& Helmus, 2007). The DRAOR is an empirically informed scale. Although there may not be specific correlational data for all items, their inclusion is suggested or supported by a review of the offender rehabilitation and risk assessment literature (Serin, 2007).

The DRAOR uses a 3-point scoring format for items ranging from 'not a problem' (0) to 'definite problem' (2) for Stable and Acute factors and from 'not an asset' (0) to 'definite asset' (2) for Protective factors. However, it does not yield a score that is statistically related to risk. Instead, the domain areas provide an outline for parole and probation officers to consistently follow during offender contacts. This encourages a focus on information related to changes in risk state, aiding parole and probation officers in responding with changes in their risk management strategies. The DRAOR also includes risk scenario planning, which aims to inform structured professional decisionmaking related to the level of parole supervision and appropriate types of intervention and management strategies. This is accomplished by identifying probable offending outcomes based on what is known about the offender and of their criminal history.

Stable risk factors. Stable dynamic risk factors are potentially changeable factors but are durable and tend to persist over time and across different settings (Serin, Mailloux et al., 2010). Change is clearly possible in these areas but will likely be somewhat gradual and may take months or years to change. There are seven variables considered under this domain related to peer associations, attitudes toward authority, impulse control, problem-solving, sense of entitlement, and attachment with others. These items reflect criminogenic needs, as described by Andrews and Bonta (2010a). 
Acute risk factors. Risk assessment tools that are optimal for the assessment of acute risk may not be the same as those for chronic risk (McNeil, Gregory, Lam, Binder, \& Sullivan, 2003); therefore both stable and acute factors should be considered for an accurate account of dynamic risk. Acute dynamic risk factors can change rapidly within days, hours, or minutes and help determine the imminence of individual reoffending (Hanson \& Harris, 2000). These factors include substance abuse, anger/hostility, opportunity/access to victims, negative mood, employment, interpersonal relationships, and living situation. Deterioration of these factors implies the offender is at a greater risk for imminent reoffending. This subscale reflects proximal indicators of risk state as described by Douglas and Skeem (2005) and answers the important question of when an offender might reoffend.

Protective factors. Protective factors are the characteristics and assets of an offender that may mitigate the probability of engaging in criminal behaviour (Serin, Mailloux et al., 2010). Variables of interest include responsiveness to advice, prosocial identity, high expectations, costs/benefits, social support, and social control. These are the internal assets and external strengths of an offender that may help reduce the risk of them committing another offence. These factors are not simply the inverse of the risk factor (e.g., antisocial attitudes) but rather positive attributes that shield or armour the individual from risky situations (e.g., prosocial attitudes; Farrington \& Loeber, 2000; Newcomb \& Felix-Ortiz, 1992). These protective factors are likely context-specific and quite dynamic in nature. It is believed that the more protective factors there are, the greater the likelihood that an offender will be resilient to the risks they experience. Although there is a paucity of literature in terms of our understanding of factors 
protective for risk of future offending in adults, these items were selected based on theory and a review of the literature several years ago (Serin, 2007). Of note, the development of the DRAOR was parallel to research by Ullrich and Coid (2011), yielding considerable item overlap.

\section{Initial Findings on the DRAOR}

The DRAOR is not a fully validated instrument, although initial findings provided evidence of its utility as a dynamic risk assessment instrument. Tamatea and Wilson (2009) conducted a pilot study in New Zealand with 7 senior probation officers and 59 offenders that they were tasked with managing. The probation officers were trained on the use of the DRAOR and supervised in its application. The offenders were released on probation between October 2008 and June 2009 and were administered at least one DRAOR during their probation contact.

Findings from this pilot study demonstrated the reliability and validity of the DRAOR. All three subscales were normally distributed and were found to change over the course of the study. Moderate positive correlations were found between the Stable and Acute subscales $(r=.52)$. In addition, the Protective subscale was negatively moderately correlated to a validated measure of static risk of reoffending, the Risk of reConviction X Risk of re-Imprisonment model (RoC*RoI; Bakker, O’Malley, \& Riley, 1998; $r=-.33)$ as well as the Stable $(r=-.46)$ and Acute $(r=-.41)$ subscales, suggesting that the protective variables may utilize different psychological processes than risk factors. This supports a pathway-type model of offending and desistance from crime. Predictive validity was established by comparing offenders who reoffended (i.e., either recalled or reconvicted) to those who did not reoffend (i.e., crime desistance group). 
Dynamic risk variables and the Protective subscale in particular were significant predictors of parole success and desistance from crime.

Supervision by the researchers revealed that probation officers had a good understanding of most of the DRAOR items and required little direction in scoring after the first month of follow-up. Feedback from the probation officers confirmed that the DRAOR was a brief but useful measure that helped both structure their interactions with offenders and simplify their role. Therefore, the DRAOR shows promise in helping probation officers focus their assessments on dynamic risk factors that are empirically related to offending and protective factors that are related to crime desistance. The measure is now fully implemented in New Zealand, but further research is needed to validate the instrument. Although the sample in the pilot study was mostly representative of the New Zealand offender population, the sample size was small.

The DRAOR was also piloted in the United States in Iowa State Probation and Parole following a one-day training on risk assessment and the use of the DRAOR (Serin \& Prell, 2012). A total of 563 offenders were assessed once on the DRAOR and 363 of those offenders were assessed a second time over a 6-month period. The mean number of days between Time 1 and Time 2 assessments was 65.6 days. Total DRAOR scores were found to increase for $42.7 \%$ of the sample (i.e., higher risk), decrease for $42.7 \%$ of the sample (i.e., lower risk), and remained the same for $14.6 \%$ of the sample. The offenders were followed up for three months following the six-month period over which assessments were made. Time 1 subscale scores were significantly correlated with any violation or return to prison (Total score $r=.28$; Stable Risk $r=.25$; Acute Risk $r=.23$; Protective Factors $r=-.28$ ). Correlations diminished but were also significant for serious 
violations and new crimes. Similar patterns of findings were revealed for Time 2 DRAOR scores. The DRAOR demonstrated moderate predictive accuracy for the total score $(\mathrm{AUC}=.66)$, Stable Risk $(\mathrm{AUC}=.60)$, Acute Risk $(\mathrm{AUC}=.65)$, and Protective Factors $(\mathrm{AUC}=.67)$, which were all slightly better than the LSI-R $(\mathrm{AUC}=.56)$. The predictive accuracy of the DRAOR was also tested by allocating cases to one of four risk bins using the Total DRAOR score ((Stable + Acute $)-$ Protective $)$. Offenders with scores in the high risk bin had failure rates twice that of the overall $16 \%$ base rate for the sample and offenders with scores in the low risk bin had failure rates one half of the base rate. Feedback from probation and parole officers regarding the DRAOR's ease of use and clinical utility was very encouraging and the Iowa Department of Corrections has now replaced the LSI-R (Andrews \& Bonta, 1995) with the DRAOR for communitybased risk reassessments.

In comparison to other dynamic risk measures, the DRAOR potentially provides a more proximal and sensitive index of change. The inclusion of protective factors that reflect contemporary desistance research is supported by initial findings that indicate that these protective factors are most predictive of re-entry success. These pilot studies provide a solid foundation to validate the DRAOR in a longitudinal study with a larger sample.

\section{Conceptual Model}

The development of the DRAOR reflects the conceptual work of a number of theories. The Personal, Interpersonal, Community - Reinforcement (PIC-R) perspective and the Risk, Needs, Responsivity (RNR) model proposed by Andrews and Bonta (2010a) provide a framework for understanding what leads offenders into offending and 
reoffending, as well as effective rehabilitation and community supervision strategies for those offenders. These theories provide an important background for the use of dynamic factors in risk assessment to improve the efficacy of offender re-entry and aftercare. Recently there has been a shift in focus to also disentangling why an offender ceases to engage in criminal behaviour. A pathway model of desistance provides a basis for justifying why the inclusion of protective factors to augment dynamic factors can aid in both risk assessment and risk management.

The PIC-R perspective, proposed by Andrews and Bonta (2010a), describes the factors that encourage or discourage criminal conduct. This model posits the occurrences of deviant and non-deviant behaviour are under the control of antecedent and consequent events. Both reinforcement (i.e., rewards) and punishment (i.e., costs) as a consequence of an act influence future behaviour. Further, rewards and costs can be additive or subtractive in that the event can be introduced or withdrawn. The strength of antecedents and consequences depend on the interaction of the individual with the environment, suggesting that there are many physical and cognitive characteristics that influence the capability to respond and learn. The probability of the occurrence of criminal conduct is therefore a positive function of the rewards associated with that behaviour and a negative function of the associated costs. Based on the PIC-R, the likelihood of offender change and desistance from crime increases as the rewards for prosocial behaviour are raised, the rewards for antisocial behaviour are diminished, and/or the costs for antisocial behaviour are raised. Components of the PIC-R are reflected in the conceptual model underlying this study, namely the role of contingencies and costs and benefits in offender behaviour. 
While the PIC-R perspective provides an understanding of what leads to criminal behaviour, the RNR model details how to effectively intervene with offenders through treatment or supervision. The importance of juxtaposing case management with the "What Works" literature has been recognized (Taxman, Shepardson, \& Bello, 2003 as cited in Bonta, Rugge, Scott, Bourgon, \& Yessine, 2008). That is, the principles of effective correctional programming are also germane for case management (what some now refer to as core correctional practice; Lowenkamp, Pealer, Smith, \& Latessa, 2006). The Risk principle of effective rehabilitation indicates that the intensity of intervention should be matched to the risk level of the offender, with intensive services reserved for high risk offenders and minimal services provided to low risk offenders (Andrews \& Bonta, 2010a). Utilizing risk/needs assessments aids parole and probation officers in determining an appropriate amount of supervision for each individual offender. Based on this principle, offenders with higher levels of risk warrant a greater frequency of contact with their probation officers. The Need principle dictates that criminogenic needs should be addressed in treatment in order to reduce the likelihood of future criminal conduct. Criminogenic needs are dynamic risk factors that should reduce risk when changed, such as antisocial attitudes, antisocial associates and substance abuse (Andrews \& Bonta, 2010a). Non-criminogenic needs, such as self-esteem, do not have a significant impact on recidivism when changed (Andrews \& Bonta, 2010a). Accordingly, dynamic risk factors are important from a risk assessment and risk management perspective given their relationship to recidivism and their potential to improve correctional outcome when reduced. These dynamic factors must be identified and targeted for change in an offender in order to reduce criminal conduct. Lastly, the Responsivity principle states that the 
mode and style of intervention must be matched to the learning style and abilities of the offender. From a community supervision perspective, this involves parole officers interacting with offenders in a therapeutic manner and employing skills and techniques supported by RNR (Bourgon \& Gutierrez, 2012; Bourgon, Gutierrez, \& Ashton, 2012).

Correctional programming adhering to the principles of RNR has been demonstrated to be successful in reducing recidivism (Andrews \& Dowden, 2005; Dowden \& Andrews, 2000; Latessa, 2004). For instance, treatment that adheres to the principles of risk, need, and responsivity has a mean effect size $(r)$ of .30 , while inappropriate treatment (i.e., treatment not adhering to the principles) has a mean effect size of -.06 (Andrews et al., 1990). Smith, Gendreau, and Swartz (2008) also found that recidivism was reduced by 28 percent (i.e., mean effect size $(r)$ of .28 ) when the principles of effective treatment are followed. However, the adherence to these principles may be lacking in the context of community supervision. For instance, in a study of probation officers and their clients, the frequency of contact was only mildly related to the offender's level of risk and officers rarely intervened to target well-known criminogenic needs such as antisocial attitudes and peers, when such an intervention may facilitate change (Bonta et al., 2008).

An offender's release from prison into the community represents a crucial phase in crime desistance. Approximately 50\% of offenders who fail parole do so in the first three months (Brown, St. Amand, \& Zamble, 2009). The predictors of recidivism also change with time (Ullrich \& Coid, 2011), with triggers (e.g., substance abuse) being more predictive immediately after release and appraisals (e.g., perceived problem level and global stress) more important a few months after release (Brown et al., 2009). The 
overarching goal of discharge planning is a coordinated and collaborative effort to ensure a continuum of care and treatment during the re-entry process (Mellow \& Christian, 2008). Effective case management requires an assessment of the needs of offenders followed by an incorporation of these needs to a service delivery plan (Healey, 1999). An understanding of "What Works" in offender rehabilitation has stimulated a shift in how offenders are supervised in the community from a traditional case management approach to that of "agents of change" (Bourgon, Gutierrez, \& Ashton, 2011; 2012). This shift comes in light of recent research that traditional supervision has little effect on recidivism (Bonta et al., 2008; Green \& Wink, 2010; Solomon, Kachnowski, \& Bhati, 2005). Probation officers have been found to focus their efforts less on the service delivery role of supervision and more on the enforcement aspect of supervision by ensuring offenders comply with their conditions of probation (Bonta et al., 2008). The role of community supervision officers has begun to be directly and actively involved in the therapeutic change process (Bourgon et al., 2011). In order to act as effective agents of change, probation officers must have the knowledge, skills, and abilities necessary to influence behavioural change in their clients.

Offenders are subject to situations while on release that may increase or decrease their likelihood of reoffending. Continually assessing and reassessing the factors which predict recidivism provides a more accurate foundation for determining ongoing intervention and supervision strategies. Potentially, improving the efficacy of re-entry requires more systematic assessment and more balance by expanding the focus to protective factors and not solely focusing on offender deficits. This will allow for a more 
refined allocation of resources through the identification of risk-dependent supervision strategies.

Improving risk management with dynamic factors. Evidence-based practice for community supervision highlights the need for a case plan that reflects an accurate assessment of risk and needs (Andrews \& Bonta, 2010b). Continuous reassessment of dynamic risk in particular allows for the identification of which offenders are at risk of reoffending and when reoffending is most likely to occur. As such, the consideration of dynamic risk factors helps detect fluctuations in intra-individual risk that may lead to an impending failure (Andrews \& Bonta, 2010a). A consideration of dynamic risk can help determine an offender's risk state - when the offender may reoffend (Douglas \& Skeem, 2005). Dynamic risk has been found to augment static risk estimates (Brown, 2002; Hanson et al., 2007), although the different aspects of risk (i.e., static, stable dynamic, and acute dynamic) are important at various points of contact (Hudson, Wales, Bakker, \& Ward, 2002).

Stable factors should be targeted in treatment or other interventions aimed at fostering enduring improvements given that they can remain unchanged for months or years (Hanson \& Harris, 2000). Stable factors have also been described as traits (Ward \& Beech, 2004), while acute factors can change more rapidly within days, hours, or even minutes. These factors show less of a relationship to longer-term risk potential, but are related to the timing of reoffending (Hanson \& Harris, 2000). Acute factors have also been described as triggering or contextual risk factors, or the state expression of traits prompted by these triggering or contextual factors (Ward \& Beech, 2004). As a result, some of the acute factors comprising the DRAOR represent the state expression of 
personality traits resulting from environmental circumstances. Given that they are more proximal, acute factors should demonstrate stronger relationships with recidivism when systematically assessing dynamic risk. However, in one of the key empirical tests of dynamic risk, The Dynamic Supervision Project, acute factors provided more information regarding relatively enduring characteristics, rather than the timing of reoffending (Hanson et al., 2007).

The designation of dynamic risk factors as either stable or acute is important, at least at a conceptual level, although there remains debate regarding appropriate terms and operationalization (see Mann, Hanson, \& Thornton, 2010). It may also be more effective for parole and probation officers to target interventions toward stable factors to create improvements in the long-term. Shifts in acute factors may act as warning signs for increased risk and should be monitored by parole and probation officers. However, the distinction between stable and acute risk factors is still in the theoretical stage and requires more refined longitudinal analyses (Jones, Brown, \& Zamble, 2010). The current study aimed to address this distinction with a number of measurement occasions in relatively close proximity, allowing for change to be more accurately depicted over time.

Focus has shifted in research and practice from risk assessment to risk management. Risk assessment requires not only an assessment of long-term risk but also sensitivity to changes in the likelihood of reoffending (Quinsey, Jones, Book, \& Barr, 2006). Unlike static factors which are not amenable to treatment, dynamic factors are changeable. Recent research on risk has attempted to identify factors that can lead to a decrease in risk if properly managed (Beech, Friendship, Erikson, \& Hanson, 2002; 
Douglas \& Skeem, 2005; Thornton, 2002). By carefully monitoring these risk indicators, parole staff may be able to provide more timely interventions, averting relapse (Hanson, 2009). On a related note, some studies indicate poor compliance between assessment and case management or action plans (Bonta et al., 2008; Harris, Gingerich, \& Whittaker, 2004). The DRAOR provides a within-session structure for systematically and constantly revising case planning and risk management. This approach may ensure a more consistent follow through between risk assessment and case management. In addition, it may be possible to motivate offenders by concentrating on modifying their dynamic risk factors (Andrews et al., 2011).

The desisting offender. Criminal careers can be viewed as a continuum from crime acquisition to desistance (Serin \& Lloyd, 2009; Serin, Lloyd et al., 2010). It is important to view the transition from being an offender to an ex-offender as a process, one that does not necessarily occur instantaneously and certainly not for all offenders. This draws parallels to the conceptualization of recidivism as a process, beginning with a precipitating environmental trigger followed by a cognitive and emotional appraisal of the situation and an attempt to deal with the situation (Zamble \& Quinsey, 1997). The complete cessation of criminal behaviour is unlikely to occur suddenly, especially among offenders who have been highly involved in criminal activities from a young age (Kazemian, 2007). Offenders with poor coping skills have trouble remedying these precipitating situations, which results in a worsening of the cycle of negative emotions, maladaptive cognitions, and ultimately reoffending (Zamble \& Quinsey, 1997). A significant amount of variability has been found in the factors examined to understand the offence process leading to recidivism (Zamble \& Quinsey, 1997). Similarly, desistance 
has been conceptualized as a change process involving multiple internal factors, tied to the psychological mechanisms that drive changes in behaviour (Maruna, 2001; Serin \& Lloyd, 2009). A pathway model of crime desistance provides the theoretical framework for this study.

The process of desistance can vary along a number of dimensions. For example, the change can be abrupt or gradual, begin early or late in the individual's criminal career, and begin at younger or older ages (Bushway, Thornberry, \& Krohn, 2003). Currently the specific factors that identify when offenders will and will not begin the transition to desistance are unclear (Serin \& Lloyd, 2009). Findings based on a transitional model of crime desistance suggest there are internal (e.g., identity/selfconcept, change beliefs) and external (e.g., correctional intervention, positive relationships) change factors which occur when an offender makes a commitment to change (Serin \& Lloyd, 2009). A transition is then possible between engaging in crime and exiting crime. Given the multidimensional nature of the process of change, it is unlikely that only one factor will account for a large amount of variance in offender change (Connors, Carroll, DiClemente, Longabaugh, \& Donovan, 1997). What is clear is that getting out of crime is a different process from getting involved in crime (Kazemian, 2007). Thus it is important to consider more than just risk factors for engaging in crime, but also factors that buffer an offender from reoffending.

Protective factors related to desistance. Determining why an individual identified at high risk for reoffending does not reoffend can help develop a better understanding of criminal behaviour. Further, assessments that measure only static and dynamic factors may produce biased predictions of reoffending (Rogers, 2000). The 
pathway of desistance requires protective factors and maintenance through risk management. It is expected that protective factors facilitate risk management by insulating an at-risk offender from re-involving himself or herself in criminal behaviour. However, protective factors are the most overlooked variable in risk assessment (Tamatea \& Wilson, 2009). Risk assessment must move beyond existing measures and consider those factors that are related to desistance. Given that social learning theory posits that offending is multiply determined (Bandura, 1969), it is important to intervene with multiple factors in order to reduce an offender's likelihood of reoffending.

Conceptually, there is a lack of agreement on the relationship between protective factors and risk. Protective factors may represent the absence of risk factors (Costa, Jessor, \& Turbin, 1999). For instance, given that substance abuse is a risk factor for reoffending, not having any alcohol or drug abuse problems would be considered a protective factor. However, this approach has been criticized for overlooking "potential differences between the risk and protective potency of specific factors" (p. 281) and has not been validated (Newcomb \& Felix-Ortiz, 1992). Protective factors may also represent the opposite end of a risk continuum with an offender potentially scoring at the high or low end of a factor but not both simultaneously (Brook, Whiteman, Gordon, \& Cohen, 1989; Hawkins, Catalano, \& Miller, 1992; Rutter, 1987; Webster et al., 2004). An example of this notion would be different ends of the spectrum of employment; a good job situation would be protective while employment difficulties would be a risk. The START (Webster et al., 2004) conceptualizes protective factors in this manner, with each item coded as a strength or vulnerability. Finally, protective factors may exist without a corresponding risk factor and an offender may score both high and low in 
different factors in the same area (Farrington \& Loeber, 2000; Newcomb \& Felix-Ortiz, 1992). An example of this conceptualization would be social support, which is qualitatively different from known risk factors such as substance abuse. Protective factors are reflected in the DRAOR and its underlying conceptual model as existing without a corresponding risk factor.

A related conceptual issue regarding protective factors is the nature of their relationship to risk. Ullrich and Coid (2011) found that protective factors did not have different effects on different levels of risk. The risk factors weakened protective mechanisms, but the independent effects of protective factors were found whether the offender was low, moderate, high, or very high risk for future violence. This is reflected in Fitzpatrick's (1997) mediation model in which the impact of risk factors on behaviour is mediated by protective factors. This suggests that the effect of risk factors on engaging in risky behaviour is not only direct, but also indirect (i.e., by reducing or weakening protective mechanisms). Fitzpatrick (1997) offered a competing buffer model in which risk factors only have an impact under certain conditions, such as when an offender lacks protective factors. According to the buffer model, recidivism is more likely to occur when risk factors are high and protective factors are low. Although these models are not directly tested in the current study, it is expected that the effects of protective factors will be independent of risk level. Furthermore, it is believed that the more protective factors that are present, the more likely the offender will be capable of dealing with risky situations regardless of their level of risk. Of note, there is little research on whether protective factors have stable effects over time or whether their influence is dependent on time. Ullrich and Coid (2011) addressed this issue and found that some protective factors 
had an influence on reduction of risk shortly after release in the community and some were not relevant until the long term. It is expected in this study that protective factors assessed at the initial assessment will be predictive of recidivism, but more proximal assessments will have the greatest accuracy.

Lastly, there is a lack of consensus on whether certain factors should be considered a protective or a risk factor. For example, the SAPROF (De Vogel et al., 2007) considers living circumstances, work, and intimate relationships as protective factors while these are categorized as dynamic risk factors in the DRAOR. The DRAOR was developed with dynamic risk factors considered as aggravating and protective factors considered as mitigating. On any given risk factor, an offender is evaluated as higher risk when it is present and lower risk when it is not, but protective factors can exist even when there are risk factors present. Therefore, an offender may have a number of aggravating circumstances by scoring high on most dynamic risk factors (e.g., antisocial peer associations), but there may also be mitigating circumstances that protect him from reoffending (e.g., availability of prosocial support system).

From this discussion, it is evident that the relationship between risk and protective factors is highly complex. Coupled with this, the inclusion of protective factors in the risk assessment and risk management process is novel and research is in its infancy. Hence, the present study will examine empirical and conceptual issues relating to protective factors, as well as dynamic risk and crime desistance. As well, this study will explore this issue by examining the underlying factor structure of the DRAOR. 


\section{Current Study}

The DRAOR is a dynamic risk assessment tool that provides a user-friendly framework. It measures both positive (i.e., protective) and negative (i.e., stable and acute dynamic risk) factors that can change quite rapidly at the individual level. Changes in these factors can help inform decisions regarding individual offenders and improve community supervision practices. As with most correctional research, the underlying goal of this study is to augment evidence-based practice by refining theory, assessment, and case supervision. The DRAOR is now fully implemented in New Zealand but has not been fully validated, although initial findings suggest it is a valid and reliable measure. Its natural implementation reflects its support by probation officers and the Department of Correction's interest in providing structure to community supervision.

The purpose of this study was to replicate early developmental research regarding this dynamic risk measure, specifically through the application of the DRAOR in the New Zealand Department of Corrections. The current research endeavour is a longitudinal study of an archival dataset from a large sample of parolees who were administered the DRAOR by trained probation officers. This study validated the DRAOR through an examination of its psychometric properties to determine if and how it can be improved (e.g., item reduction). This addressed whether the DRAOR can be reduced to a more parsimonious scale to yield greater accuracy.

The study tracks the sample's reconviction records across a two-year period to examine the DRAOR's accuracy in predicting recidivism. First, offenders with a reconviction were compared to those who succeeded over the two years on their dynamic risk and protective factor scores. Reconvicted offenders were hypothesized to have 
higher dynamic risk, lower protective factors, and higher overall DRAOR scores, in agreement with pilot research (Tamatea \& Wilson, 2009). Reconvicted offenders were examined to determine whether changes in their dynamic risk levels or protective factors in the time leading up to their failure were incrementally predictive. It was expected that the protective factors would be the most predictive, consistent with the transitional model. Acute dynamic factors were hypothesized to demonstrate greater accuracy than Stable dynamic factors given that they are more proximal. The predictive validity of the DRAOR was also tested compared to a static risk assessment scale (RoC*RoI). The $\mathrm{RoC}^{*} \mathrm{RoI}$ is predictive of recidivism (Bakker et al., 1998), therefore the DRAOR was expected to show evidence of good convergent validity with this tool and add incremental validity. Consistent with prior research (Brown, 2002), it was expected that a model combining the dynamic risk factors and protective factors of the DRAOR and the static risk factors of the RoC*RoI would demonstrate the greatest accuracy.

Finally, this study included a theoretical examination of change in dynamic risk factors modeled over time. This line of research investigated whether or not dynamic factors actually changed over a period of systematic assessment and reassessment. It evaluated the point at which the DRAOR becomes predictive of recidivism (i.e., from the first assessment or closer to the time of reoffending). The highest accuracy was hypothesized to be observed with the assessments just prior to reoffending. These research goals were developed to advance our understanding of risk assessment and protective factors, as well as the crime desistance process.

The New Zealand Department of Corrections (2007) reports that their Maori population is over-represented in the criminal justice system, including a disproportionate 
number of prosecutions, convictions, and reconvictions. Although some of the disproportionality can be attributed to bias operating within the criminal justice system, most is related to higher levels of known risk factors rather than ethnicity. Some of the early influences and socioeconomic factors that explain Maori involvement in criminal activity may also be associated with weakened protective mechanisms later in life. Given their higher levels of risk and need and potentially lower levels of protective factors, Maori offenders were examined separately. This approach was also taken to assess the validity of the DRAOR in Maori offenders in light of their unique historical and cultural factors.

\section{Research Questions}

This research includes the following research questions and hypotheses:

Question 1a. Is the DRAOR Version 1 valid and reliable?

Question 1b. Can the DRAOR Version 1 be improved to render it more reliable and/or valid?

Hypothesis 1. The DRAOR will demonstrate good psychometric properties (e.g., factor structure, Cronbach's $\alpha$, item-total correlations).

Question 2. Are there differences between reconvicted offenders and those who were not reconvicted?

Hypothesis 2. Offenders with a reconviction will have higher stable and acute dynamic risk, lower protective factors, and higher DRAOR total scores.

Question 3. Can recidivism be predicted from dynamic and protective withinoffender variables? 
Hypothesis 3a. Recidivism can be predicted from stable and acute dynamic risk factors.

Hypothesis 3 b. Desistance from crime (i.e., no reconvictions during follow-up) will be best predicted from protective factors.

Question 4. Does the DRAOR demonstrate convergent validity with static risk?

Hypothesis 4. The DRAOR will demonstrate good convergent validity with the RoC*RoI.

Question 5. Does the assessment of dynamic risk (using the DRAOR) improve predictive validity above and beyond static risk?

Hypothesis 5. The DRAOR will predict recidivism above and beyond the RoC*RoI, but the combined dynamic and static model will demonstrate the greatest accuracy.

Question 6. Do dynamic factors change over a period of systematic assessment and reassessment?

Hypothesis 6. Dynamic factors will change over a period of systematic assessment and reassessment within an individual offender.

Question 7. At what point does the DRAOR become predictive of recidivism?

Hypothesis 7. The DRAOR will significantly predict recidivism from the first assessment, with higher accuracy just prior to reoffending. 


\section{Method}

\section{Participants}

Sample selection. The sample consisted of all offenders released on parole following a term of incarceration in New Zealand from April 1, 2010 to March 31, 2012. To allow for comparison with international literature, parole refers to community supervision by a probation officer following a sentence of imprisonment. In New Zealand, all offenders sentenced to imprisonment for two years or more are released from prison into a period of supervision by a probation officer for a minimum of six months. Offenders are "released on conditions" if they are released at their sentence end date and standard conditions are imposed (e.g., report in person to a probation officer, reside at an approved address). Offenders granted "parole" before their sentence end date may have additional special conditions attached (e.g., attend a post-release Board hearing to monitor compliance, remain at a specified residence, submit to electronic monitoring, undertake specific treatment programs).

Participants were not pre-selected by any other criteria to allow for comparisons by type of recidivism and Maori versus non-Maori. Although information was provided on parolees, the research instrument does not include any information that is not routinely considered and/or available from interview and file information. Nonetheless, identifiers were stripped by the New Zealand Department of Corrections research personnel prior to providing the data to the investigator.

Sample characteristics. A total of 3498 parolees were included in the study, and most were male (92.9\%). The 246 female parolees were grouped into the analyses for all offenders, given that this is the first comprehensive study to examine the DRAOR in New 
Zealand, with the exception of the small pilot study. Participants ranged in age from 17 to 87 years, with a mean age of 35.5 years $(S D=11.6)$. As Table 1 illustrates, approximately half of the sample was Maori (52.1\%). At the time of release, most offenders had never been married (65.7\%). An additional 196 offenders were released on parole during the study period but no data was available regarding their static risk, DRAOR assessments, or recidivism. As a result, they were not included in any analyses. Table 1

\section{Sample Demographics}

\begin{tabular}{lcc}
\hline Variable & $\%$ & $n$ \\
\hline Most Recent Self-identified Ethnicity & 52.1 & 1824 \\
Maori & 37.3 & 1308 \\
European & 7.5 & 261 \\
Pacific Peoples & 2.0 & 71 \\
Asian & 0.8 & 29 \\
Other Ethnicity & 0.1 & 5 \\
Unknown & & \\
& & \\
Marital status at release & 65.7 & 2299 \\
Never been married & 16.4 & 574 \\
Married/common law relationship & 11.1 & 389 \\
Separated/divorced & 6.1 & 213 \\
Unknown & 0.7 & 23 \\
Widowed & & \\
\hline
\end{tabular}

Slightly less than half of the sample (44.3\%) was incarcerated for a non-violent offence, such as property abuse or damage, driving and traffic offences, drugs or alcohol related, or administrative. The remaining proportion of offenders was incarcerated for non-sexual violent offences (39.3\%) and sexual offences (16.4\%). A detailed breakdown of the characteristics of the Most Serious Offence is displayed in Table 2. In terms of the seriousness of the offences, the majority of the offences were deemed to be moderate 
$(56.3 \%)$ or high $(34.8 \%) .{ }^{1}$ For those with determinate parole sentences $(97.7 \%)$, the mean sentence length was 462 days $(S D=299)$, with a range of 1 to 2422 days. $^{2}$ A small proportion of the sample $(2.3 \%)$ was released on a life sentence of parole.

Table 2

Most Serious Offence Characteristics Related to Conviction

\begin{tabular}{lcc}
\hline Variable & $\%$ & $n$ \\
\hline Offence Category and Group & & \\
Non-violent offence & 44.3 & 1550 \\
Dishonesty & 25.0 & 873 \\
Drugs and antisocial offences $_{\text {Property abuses and damage }}$ & 14.4 & 502 \\
Driving offences & 2.1 & 75 \\
Administrative and miscellaneous justice & 1.2 & 40 \\
Alcohol related & 1.0 & 36 \\
Non-sexual violent offence & 0.7 & 24 \\
Sexual offence & 39.3 & 1375 \\
& 16.4 & 573 \\
Offence Seriousness Group & & \\
High & & 1217 \\
Moderate & 34.8 & 1970 \\
Low & 56.3 & 246 \\
Lowest & 7.0 & 65 \\
\hline
\end{tabular}

${ }^{1}$ Dishonesty offences consist of theft, fraud, and burglary.

\footnotetext{
${ }^{1}$ The most serious charge is selected according to the Ministry of Justice seriousness score, which is described as: "A seriousness of offence scale was originally developed by the Policy and Research Division of the Department of Justice in 1991, and has been updated about every five years since then. The most recent update of the scale occurred in February 2005 by the Ministry of Justice. The updated scale gives imprisonable offences a score according to how serious judges have deemed each offence in terms of the use of custodial sentences over a specific time period. The updated scale is based on court sentencing data for the period 2000 to 2004. The seriousness score assigned to each offence is the average number of days of imprisonment imposed on every offender convicted of that offence between 2000 and 2004, where the average is taken over both imprisoned and non-imprisoned offenders. Suppose, for example, that between 2000 and 2004 there were 100 cases of offenders convicted of a particular offence. Of these cases, 50 resulted in a custodial sentence, and the average length of the custodial sentences imposed on these offenders was 30 days. The seriousness score for this offence is $(30 \times 50 / 100)$, or 15 . Offences that became obsolete prior to 2000 were given the same score as any new similar offences, or a score was calculated based on sentencing data before 2000. Imprisonable offences for which there were convictions but no custodial sentences over the period 2000 to 2004 , were given a seriousness rating slightly lower than the least of the offences already assigned a seriousness score (i.e. a score of 0.2 ). Non-imprisonable offences were assigned a seriousness score of zero" (New Zealand Department of Corrections, 2011, para. 3).

${ }^{2}$ There were 14 cases with a sentence length less than six months (the minimum period of supervision).
} 
Based on a static risk assessment prior to release (i.e., RoC*RoI), ${ }^{3}$ a large percentage of the sample was rated a low risk to reoffend $(43.5 \%, n=1521)$, indicating a less than $50 \%$ probability of going to prison in the next five years. A greater-than-even probability of returning to prison was assigned to a considerable proportion of offenders in the sample considered medium risk $(22.3 \%, n=782)$. About a third of offenders were rated a high risk with likely imprisonment within 5 years $(33.0 \%, n=1153)$. Few offenders were rated a very high risk with a greater than $90 \%$ probability of returning to prison $(0.9 \%, n=32)$. During the two-year follow-up period, 1516 offenders $(43.3 \%$ of the sample) reoffended. The vast majority of the reconvictions were for non-violent offences and most of these convictions were rated lowest and low in terms of seriousness (see Table 3).

Table 3

Most Serious Offence Characteristics Related to Reconviction

\begin{tabular}{lcc}
\hline Variable & $\%$ & $n$ \\
\hline Offence Category and Group & & \\
Non-violent offence & 89.2 & 1353 \\
Administrative and miscellaneous justice & 21.5 & 754 \\
Dishonesty & 6.3 & 221 \\
Driving offences & 3.4 & 121 \\
Drugs and antisocial offences & 3.4 & 118 \\
Alcohol related & 2.2 & 77 \\
$\quad$ Property abuses and damage & 1.7 & 62 \\
Non-sexual violent offence & 10.4 & 158 \\
Sexual offence & .003 & 5 \\
Seriousness Group & & \\
Lowest & & 1189 \\
Low & 78.4 & 146 \\
Moderate & 9.6 & 132 \\
High & 8.7 & 12 \\
\hline
\end{tabular}

\footnotetext{
${ }^{3}$ RoC*RoI scores were missing for 10 offenders in the sample.
} 


\section{Procedure}

As of April 1, 2010, the DRAOR was fully implemented by the New Zealand Department of Corrections for all offenders released on parole. Each probation officer has a caseload of offenders who they see regularly as part of the conditions of supervision. For every "quality" (i.e., meaningful and sufficient time) contact between the probation officer and the offender, the DRAOR is administered. Interviews typically review offenders' progress in terms of employment, relationships, treatment programs, and living situations. Depending on the risk level of the offender and how long he or she has been released from prison, they could be interviewed in the range of twice weekly to every two weeks. The number of quality contacts between offenders and their parole officers ranged from 1 to $120(M=27.8, S D=16.7)$. Table 4 displays the frequency of contact for three-month increments beginning at parole start date.

Training was provided to probation officers regarding risk assessment in general, dynamic risk assessment and the use of the DRAOR as part of ongoing staff development. All probation staff attended a one-day workshop that included practice scoring of the DRAOR during implementation. The training was delivered by trained trainers using standardized workshop and training materials. Extra supervision was provided during the data collection process since the implementation of the DRAOR to ensure a consistent standard of administration. Probation staff had the availability of Subject Matter Experts (SMEs) to supervise application of the measure during the first 12 months of implementation and address integrity issues on a permanent bases. The SMEs received advanced training from one of the scale developers, as well as supervision monthly for the first two years. Probation staff also have access to a website that 
includes a practice centre for DRAOR issues and includes extra resources including relevant articles.

Although probation officers were supposed to apply the DRAOR beginning on April 1, 2010, implementation was sporadic for the first few months as they adjusted to its use. The total number of assessments for all offenders increased each month after the parole start date beginning with 591 assessments in April 2010 and ending with 5741 assessments in March 2012. The total assessments per month levelled off to around 5000 assessments per month from March 2011 onwards.

Table 4

Frequency of Contact between Probation Officers and Parolees $(N=3498)$

\begin{tabular}{lcccc}
\hline \multicolumn{1}{c}{ Time Period } & $n$ & Range & $M$ & $S D$ \\
\hline Parole start date -3 months & 3449 & $0-45$ & 11.25 & 5.00 \\
$3-6$ months & 2850 & $0-34$ & 6.30 & 4.57 \\
$6-9$ months & 1946 & $0-33$ & 3.64 & 4.14 \\
$9-12$ months & 1431 & $0-26$ & 2.58 & 3.76 \\
$12-15$ months & 985 & $0-24$ & 1.70 & 3.21 \\
$15-18$ months & 657 & $0-24$ & 1.05 & 2.62 \\
$18-21$ months & 356 & $0-23$ & .50 & 1.84 \\
$21-24$ months & 118 & $0-12$ & .12 & .80 \\
\hline
\end{tabular}

Most of the analyses make use of all available DRAOR scores that were collected for each quality contact between the probation officer and offender. It is possible that some offenders were already in the community at the study start date and began being assessed on the DRAOR once it was implemented. Some offenders were also recalled or reconvicted after release, served a short sentence and then were released on a further period of parole. In general, all assessments were utilized beginning from the April 1, 2010 DRAOR implementation date and study start date up until either the first reconviction for recidivists or the study end date of March 31, 2012 for non-recidivists. 
This approach took advantage of more assessments per offender to accurately capture changes in dynamic risk factors and protective factors over time. For analyses where the nuance of start and end dates were more important (e.g., Cox regression survival analysis), assessments were only included if they occurred after the start date of the first parole term during the study period.

In addition, scores for each inmate on the RoC*RoI (Bakker et al., 1998), a static risk assessment measure similar to Canada's Statistical Information on Recidivism Scale (SIR-R1; Nuffield, 1989), were collected to allow for comparisons of static and dynamic risk. Demographics (e.g., age, ethnicity, gender) and information related to the offender's criminal history and incarceration (e.g., index offence type, parole sentence length) were gathered. Lastly, follow-up outcome data was collected from April 1, 2010 to July 18,2012 . This outcome data is in the form of reconviction, whether a parole failure or new conviction. The mean follow-up period (from parole start to study end date) per offender was 481 days $(S D=207$ days $)$, with a range of 112 to 834 days. The mean time-at-risk (from parole start to recidivism date for recidivists, or study end date for non-recidivists) was 312 days $(S D=228$ days), with a range of 0 to 834 days.

All data collection, coding, and entry were completed by staff of the New Zealand Department of Corrections. No recruitment efforts were required given that the use of the DRAOR is now a routine duty of the probation officers. Informed consent and debriefing forms were not administered since the data was collected for offender management and program evaluation by the New Zealand Department of Corrections. Identifying information is necessary to the study in order to combine data collected at different time points (i.e., release, community supervision, recidivism). However, the 
data were stripped of identifiers and assigned a unique identification number to merge data regarding incarceration and parole to outcome (i.e., reconviction) data.

\section{Materials}

Dynamic Risk Assessment for Offender Re-entry. The DRAOR is a clinical rating scale that considers Stable (6), Acute (7), and Protective (6) factors. The domain areas provide an outline for parole and probation officers to consistently follow during offender contacts. The DRAOR uses a three-point scoring format for each of these factors. Stable factors primarily address criminal orientation and impulsivity, while Acute factors address disinhibitors and lifestyle stressors. Each Stable and Acute factor is rated as "no problem" (0), "slight/possible problem" (1), or "definite problem" (2). Possible values range from 0 to 12 for the Stable factor and 0 to 14 for the Acute factor. Protective Factors address social support and prosocial identity changes. Each Protective factor is rated as "item not protective" (0), "slight/possible asset" (1), or "definite asset" (2) and total Protective Factor scores can range from 0 to 12. Each item can also be omitted if the evidence is unreliable. For the purpose of examining the overall DRAOR, a total score was calculated by adding Stable and Acute subscale scores and subtracting the Protective subscale score. Total scores can range from -12 to 26, with lower scores indicating lower risk and greater Protective Factors and higher scores indicating higher risk and fewer Protective Factors.

The time taken to apply this scale is approximately 20 to 30 minutes, depending on the user's familiarity with the instrument, the complexity of the case, and whether it is a baseline or repeated assessment. The number of DRAOR assessments for each offender varies depending on their risk level and how long they have been in the 
community. The DRAOR is not a fully validated instrument, although initial findings provide evidence of its utility as a dynamic risk assessment instrument in New Zealand. In a pilot study using 7 senior probation officers and 59 offenders they were tasked with managing, Tamatea and Wilson (2009) found that each of the subscales were normally distributed and changed over the course of the study. Both initial ratings and the most recent ratings on Acute Risk and Protective Factors were significant predictors of parole success. The DRAOR demonstrated good convergent validity with the RoC*RoI, particularly the Protective subscale $(r=-.33)$.

\section{Stable risk factors.}

Peer associations. The nature and frequency of peer associations is an important variable that can be considered as positive or negative. Having only antisocial peers or being involved in a gang is problematic (score of 2), while having only prosocial peers is not a problem (score of 0 ). Antisocial associates have been demonstrated to have one of the strongest correlations to criminal behaviour $(r=.21)$, and are considered one of Andrews and Bonta's "Big Four" predictors of crime (Andrews \& Bonta, 2010a). It is included in a number of other risk assessment tools including the LSI-R (Andrews \& Bonta, 1995), which measures whether an offender has some criminal acquaintances or friends and few anti-criminal acquaintances or friends.

Attitudes towards authority. This item captures the offenders' view of others, particularly in receiving guidance or direction from them. Offenders with an antagonistic attitude towards others, especially those in positions of authority, are considered to have an increased risk of reoffending. A similar item is included in the LSI-R (Andrews \& Bonta, 1995) with authority interaction, but only in relation to school or employment. 
This is also reflected in the STABLE-2007 in terms of the offender's cooperation with supervision and specifically how the offender is working with the officer (Hanson et al., 2007). Antisocial attitudes in general appear to be malleable, with a lack of change in criminal attitudes related to disciplinary infractions while incarcerated (Walters, Trgovac, Rychlec, DiFazio, \& Olson, 2002).

Impulse control. Impulsive offenders with poor self-regulation are considered to have a higher risk of reoffending (Andrews \& Bonta, 2010a). Impulsivity acts as a disinhibitory factor and is marked by 'spur of the moment' actions without taking into consideration the consequences (Van Voorhis, 1997). On a larger scale, desistance from crime involves breaking a pattern of criminal behaviour and exercising control over impulses to commit further criminal acts (Serin \& Lloyd, 2009). Offenders who are able to be reflective, self-monitor, and make decisions independently are considered more stable. Impulsivity is captured in the "Big Four" risk factors of crime within antisocial personality as well as being adventurous, pleasure seeking, restlessly aggressive, and irritable $(r=.22$; Andrews \& Bonta, 2010a). Impulsivity has been found to predict future violent behaviour in psychiatric patients (Monahan et al., 2001), as well as distinguish correctional offenders from community controls and college students (Barratt, 1994) and recidivistic from non-recidivistic sexual offenders (Prentky, Knight, Lee, \& Cerce, 1995). The STABLE-2007 also measures impulsivity as a stable risk factor for sex offenders (Hanson et al., 2007).

Problem-solving. The ability to make good or effective decisions is rated based on whether an offender considers all the consequences or does not consider them at all. An offender should demonstrate logic in arriving at a decision, an ability to clarify a 
problem, consider a range of responses, weigh the opinion of others, and consider the consequences before implementing a solution. Effective problem solving is regarded as a skill, one that successfully moves an offender from encountering a problem to applying an appropriate solution, which is a situation-specific coping response or response pattern (McGuire, 2005). A review of the empirical evidence suggests a link between deficits in social problem solving and criminal activities or offending (Antonowicz \& Ross, 2005; Zamble \& Quinsey, 1997), and as such, a number of treatment programs have been developed to target this cognitive skill deficit. Poor problem solving skills are measured by the STABLE-2007 as a stable risk factor for sex offenders (Hanson et al., 2007).

Sense of entitlement. This item considers whether offenders have an inflated sense of self worth in comparison to a realistic recognition of their limitations. Entitlement is a criminal thinking style that is often associated with other criminal cognitions (Walters \& White, 1989). An elevated sense of entitlement is often accompanied by a belief that they are different from other offenders and that their rights are superior to others. As opposed to disregarding the rights and feelings of others in accordance with their own self-interests, an offender that has desisted from crime lives thoughtfully and with an awareness of the rights of others (Maruna, 2001). Personal accountability, a broader offender competency that reflects a sense of entitlement, has been found to predict correctional program performance (Hanby, Serin, \& Vuong, 2009) and differentiate violent offenders and other higher risk violent offenders (i.e., persistently violent offenders) from other offenders (Hanby \& Serin, 2010). A sense of entitlement has been observed to correlate to general and violent recidivism (Mills, Kroner, \& Hemmati, 2004). 
Attachment with others. The purpose of this item is to assess the extent to which an offender is connected to and concerned about others. Offenders who are callous and indifferent towards others are seen as problematic. The STABLE-2007 captures elements of this item with the measurement of a lack of concern for others and general social rejection (Hanson et al., 2007). The LSI-R considers whether the offender is a social isolate (Andrews \& Bonta, 1995), which is one aspect of this item. Other aspects include whether the offender finds it difficult to confide in others and is unable to attend to the emotional consequences of their actions. In a study of male adolescent offenders, desistors (participants who did not reoffend within a two-year follow-up) were more bonded to conventional society on measures of attachment and commitment to school and attachment to conventional peers than those who continued offending with varying levels of frequency (Ayers et al., 1999).

\section{Acute risk factors.}

Substance abuse. The purpose of this item is to measure current addictions and substance abuse, ranging from maintaining sobriety or social use to problematic abuse. Abuse of alcohol and drugs are related to crime $(r=.11)$, and are included in the "Central Eight” predictors of crime (Andrews \& Bonta, 2010a). Accordingly, alcohol and drug problems are assessed in a number of risk assessment measures including the LSI-R (Andrews and Bonta, 1995), the ACUTE-2007 (Hanson et al., 2007), and the Federal Post Conviction Risk Assessment (PCRA; Johnson, Lowenkamp, Vanbenschoten, \& Robinson, 2011). Intoxication and use of substances can change relatively rapidly, and affect other dynamic factors such as employment and interpersonal relationships. Maintenance of sobriety and the avoidance of drugs are vital to the desistance process 
(Hussong, Curran, Moffitt, Caspi, \& Carrig, 2004), with a greater chance of success the longer the recovery.

Anger/hostility. This item captures whether an offender displays hostility, in the form of antagonism towards others, or anger, in the form of emotional volatility. Hostility is a disinhibitory factor which reduces self-regulation and problem-solving skills, thereby predisposing an offender to an antisocial outcome. It is reflected in both the STABLE-2007 and ACUTE-2007 (Hanson et al., 2007). The relationship is complex, with some research identifying anger as an important influence on violent offending for some offenders (Howells, 2004). Other evidence asserts that violent offenders do not differ from non-violent offenders on measures of anger (Loza \& Loza-Fanous, 1999b). Moreover, no significant differences were found between offenders with different levels of risk scores on measures of anger (Loza \& Loza-Fanous, 1999a). Consistent with this study, anger scores were not significantly related to post-release performance (Mills \& Kroner, 2003). Although anger may be an insufficient explanation for violence on its own (Polashek \& Collie, 2004), it remains one of a number of personality attributes and emotions that may be related to reoffending; therefore it remains a legitimate item to include. Anger has been observed to be dynamic (Hanson \& Harris, 2000) and changeable as a result of treatment (Deffenbacher, Oetting, \& DiGiuseppe, 2002).

Opportunity/access to victims. This item considers whether an offender has opportunity or access to victims, for those offenders with a preferred victim or pattern of victim selection. Examples include a sex offender living near a school or a domestic abuser in a relationship. Victim access and crime opportunity can destabilize good intentions of offenders, and therefore increases the likelihood of recidivism. Access to 
victims is scored as a definite problem, while avoidance of preferred victims is scored as not a problem. Opportunity for victim access is recognized as a risk factor and included in other measures of dynamic risk, such as the ACUTE-2007 (Hanson et al., 2007) and the Dynamic Risk Assessment and Management System (DRAMS; Lindsay et al., 2004).

Negative mood. The purpose of this item is to determine whether an offender has either acute negative mood or a continued presence of negative mood (score of 2). Low negative affect or mood involves a state of calmness and serenity, while negative affect can include a variety of aversive mood states such as contempt, guilt, fear, and nervousness (Watson, Clark, \& Tellegen, 1988). Negative mood in the form of depression and anxiety has been found to differentiate recidivists and ex-offenders (Zamble \& Quinsey, 1997) and to be highly predictive of later violence in the community (Freese, Hiscoke, \& Hodgins, 2002). Strong emotional states including anxiety, depression, and anger have also been reported in sexual offenders prior to relapse (Pithers, Kashima, Cumming, Beal, \& Buell, 1988). An offender may experience negative mood as a result of heightened levels of stress. Accordingly, mood and affect appear to change over the short- and long-term (Douglas \& Skeem, 2005). Negative emotionality is represented as a stable risk factor in the STABLE-2007 (Hanson et al., 2007).

Employment. Employment stability and job satisfaction is important in sustaining crime desistance (Uggen, 1999, 2000). Maintaining a preferred job is not considered a problem (score of 0), while being unemployed is a risk factor (score of 2). Employment on its own does not manage risk, but instead there must be an appropriate match to offender skills and expectations (Andrews \& Bonta, 2010a). This item is reflected in the 
"Central Eight" risk factor of problems in school and/or work in terms of poor performance or low levels of satisfaction in either $(r=.15)$. Employment is included in a number of risk assessment tools including the PCRA in the form of being unemployed or a having poor work outlook (Johnson et al., 2011) and the LSI-R in the form of currently or frequently unemployed, ever fired, or never employed for more than a year (Andrews \& Bonta, 1995).

Interpersonal relationships. This item captures whether an offender is in a stable and healthy close or intimate relationship, a conflicted relationship, or does not have a current stable relationship. At the extreme, poor interpersonal relationships may be marked by violence and victimization. Marital engagement and satisfaction, on the other hand, are related to lower rates of offending (Maume, Ousey, \& Beaver, 2005; Sampson \& Laub, 1990). Interpersonal relationships are considered under the domain of family and marital relationships in the "Central Eight" risk factors for crime, in terms of poor family relationships ( $r=.18$ Andrews \& Bonta, 2010a). It is also reflected in the LSI-R in terms of dissatisfaction with marital or equivalent situation and a criminal family/spouse (Andrews \& Bonta, 1995).

Living situation. Given that lifestyle instability is related to reoffending and other negative outcomes (Andrews \& Bonta, 2010a), the purpose of this item is to assess whether the offender has an unstable living situation or lack of accommodation (score of 2). For example, lifestyle instability (i.e., moves, unemployment, and youthfulness) was found to be related to attrition from a male batterer treatment program (Cadsky, Hanson, Crawford, \& Lalonde, 1996). This item is scored as not a problem if the offender is living in a stable and positive living situation (score of 0 ). For instance, a positive living 
situation (i.e., living with parents) was significantly related to graduation from a treatment program for adolescent sex offenders (Seabloom, Seabloom, Seabloom, Barron, \& Hendrickson, 2003). Living situation is considered in the LSI-R in terms of unsatisfactory accommodation, frequent address changes, and living in a high crime neighbourhood (Andrews \& Bonta, 1995).

\section{Protective factors.}

Responsive to advice. Offenders who conscientiously follow direction from positive influences such as prosocial peers, probation officers, community services providers, and work supervisors, are considered to have a definite asset. Offenders who are resistant towards advice and direction may not be ready to change (Prochaska, DiClemente, \& Norcross, 1992). Evidence of behaviour change is important when assessing responsiveness, as opposed to just listening and acting in agreement with staff.

Prosocial identity. A legitimate shift in identity to being prosocial may mitigate the probability of engaging in offending. Evidence of this behaviour change might be seen in leaving a criminal gang, disengaging themselves from antisocial peers, and supporting their family (e.g., assisting with education or sporting activities of children) or community (e.g., volunteer activities). Prosocial behaviours are encouraged and developed through treatment programs; particularly those that attempt to improve cognitive skills (Young, 2010). Prosocial involvement is also recognized as a protective factor against violence for adolescents in the SAVRY (Borum et al., 2006). This item reflects Maruna's (2001) work on prosocial identity.

High expectations. High expectations regarding rehabilitation and reintegration held by both the offender and their support network can help the offender to reduce their 
risk of committing another offence. Social support has proven to be an important predictor in relation to incarcerated substance abusers' expectations of their success after release (Lemieux, 1998). As such, this item captures the level of encouragement, engagement and commitment by a support network, as well as the extent to which they provide the offender with hope. The expectations must be reasonable and a feasible plan should be in place to reach these expectations.

Costs/benefits. This item considers whether the offender demonstrates that prosocial behaviour is more important and rewarding than criminal behaviour. Examples include an offender who no longer socializes with criminal peers because he does not want to jeopardize his new job or relationship, or an offender who sets up a savings account towards the purchase of a valued item or goal. Aspects of this item are also included in the LSI-R with the measurement of attitudes or an orientation supportive of crime and unfavourable toward convention (Andrews \& Bonta, 1995). It is also captured in antisocial cognitions, one of the "Big Four" factors related to criminality, identified by Andrews and Bonta (2010a). Antisocial cognitions include attitudes, values, beliefs and rationalizations supportive of crime.

Social support. Another positive attribute that steels an offender from the risks they experience is meaningful and accessible prosocial supports. This includes partners, employment, standard leisure activities, and community programming. Social or personal support is an important protective factor and is considered in other risk assessment tools including the LSI-R (Andrews \& Bonta, 1995), the PCRA (Johnson et al., 2011), the START (Webster et al., 2004), SAVRY (Borum et al., 2006), and the SAPROF (De Vogel et al., 2007). Social support has demonstrated to have protective 
effects for violence after release, as well as emotional support and spending spare time with family or friends (Ullrich \& Coid, 2011). The LSI-R captures leisure activities by measuring the absence of recent participation in an organized activity and whether the offender could make better use of time (Andrews \& Bonta, 1995). Leisure activities alone have not been found to be predictive of recidivism (Austin, Coleman, Peyton, \& Johnson, 2003). However, this item considers the availability of a support system that can consist of a number of prosocial supports including standard leisure activities.

Social control. A strong attachment to an offender's prosocial supports is a measure of social control. An offender may have meaningful and accessible prosocial supports but without sufficient attachment, they may not be adequately protective. Offenders are considered to have a definite asset if they have strong internalized bonds with prosocial models and demonstrate compliance and cooperation with these models. Social network factors have been found to have protective effects in the long-term, but also closeness to others has demonstrated a protective effect for violence after release (Ullrich \& Coid, 2011). Strong attachments and bonds is a protective factor included in the SAVRY (Borum et al., 2006).

RoC*RoI. The RoC*RoI is a static measure of risk created by Bakker and colleagues (1998). It was developed for the New Zealand Department of Corrections to assist in the accurate prediction of an offender's risk of conviction and likelihood of reimprisonment over a five-year period post-release. The measure is based on static predictors from criminal history and demographic information. It expresses the likelihood that an offender will both be reconvicted in the future and be sentenced to a term of imprisonment for that offence. The RoC*RoI calculations produce scores that 
range from 0 (low) to 1.0 (very high). A score of .00 to .49 indicates a low level of risk and less than $50 \%$ probability of going to prison in the next five years. Scoring in between .50 and .64 suggests a medium risk level and greater-than-even probability of returning to prison. A high level of risk and likely imprisonment within five years is assigned to scores between .65 and .89 . Lastly, a score between .90 and 1.00 indicates a very high risk level and greater than $90 \%$ probability of returning to prison within five years. A listing of the items is presented in Appendix A. The $\mathrm{RoC}^{*} \mathrm{RoI}$ is routinely conducted on all individuals who engage with the New Zealand Department of Corrections and has demonstrated good predictive validity $(\mathrm{AUC}=.76$; Bakker et al., 1998).

Recidivism. The recidivism outcome data is in the form of reconvictions ${ }^{4}$ from April 1, 2010 to July 18, 2012. This follow-up period overlaps with the data collection period, but continues collecting recidivism data for almost four months after the last DRAOR assessment was collected. Information was collected on the type of reconviction (e.g., criminal or administrative), the date of failure, and the offence type. For offenders with more than one reconviction, only assessments leading up to the first reconviction were included. Although this resulted in the loss of data following the first recidivism event, the vast sample size precluded any concerns regarding power. In addition, this approach was chosen as subsequent assessments may have been contaminated, either due to intervention from the probation officer or due to skewed evaluations based on the knowledge the offender had incurred an earlier reconviction.

\footnotetext{
4 "Reconviction" was selected as the most appropriate term to describe the recidivism data. In some jurisdictions, this type of recidivism may be viewed as a "revocation" or "parole failure." Given the differences in community supervision in New Zealand, these terms do not capture recidivism events in which the parolee does not return to prison. In addition, this term was selected to account for recidivism events that occurred after an offender's parole end date but before the study end date.
} 
Analyses were performed on any reconviction type versus criminal reconviction only.

Criminal reconvictions consisted of violence, sexual offence, property offences (i.e., property abuse, property damage), substance offences (i.e., drug possession, produce/manufacture/distribute), dishonesty (e.g., theft, fraud), and driving offences (e.g., drive while disqualified, failure to obey officer). Any reconvictions includes these criminal offence groups as well as administrative offences resulting in a conviction (e.g., breach parole conditions).

Offenders may have failed parole by breaching release conditions (e.g., warning without conviction if probation officer uses discretion) or being recalled to prison during the period of study (e.g., if Board is concerned over undue risk without a breach conviction), but these instances were not counted as recidivism. ${ }^{5}$ Non-compliance and failure to meet a special condition were also not included as reconvictions given that they are considered less serious reconvictions. ${ }^{6}$ In other words, parole failures were only counted if there was a new conviction (for violating parole or for committing a new offence).

\footnotetext{
${ }^{5}$ Standard release conditions include reporting to the Community Probation Service, any restrictions on living and work arrangements, and any restrictions on associating with people. The offender may be convicted by the court for not meeting the conditions of release, resulting in a fine, a community-based sentence or another prison sentence. If the offender breaches their release conditions and was released before their statutory release date or released from an indefinite prison sentence, he or she can be recalled to prison. An offender may also be recalled because they have committed an offence punishable by imprisonment or represent a risk to the safety of the community, or any person, or class of persons. A recall to prison can also occur when the offender is subject to residential restrictions and either they are endangering the safety of anyone at their residence, the residence is no longer available, or they no longer wish to be subject to residential restrictions (Parole Act, 2002).

${ }^{6}$ Non-compliance is a failure to comply with a requirement of the sentence (e.g., failure to report as instructed, advise probation officer of a change in address), resulting in a sanction (e.g., verbal warning, written warning) rather than legal action. Special conditions may be imposed to reduce the risk of reoffending, to assist rehabilitation and reintegration into the community, or to provide for the reasonable concerns of victims. Examples of a failure to meet a special condition include refusing to participate in treatment, personal development or rehabilitative programs, spending time with persons that they have been prohibited from associating with, and contacting the victims of their offence (Parole Act, 2002).
} 
Demographic information. Demographic information such as the age, gender, and ethnicity of each offender was gathered from New Zealand's Integrated Offender Management System (IOMS). Given the Aboriginal population of Maori peoples, comparisons were made between Maori and non-Maori offenders.

Index offence information. Data were also obtained from the computerized IOMS on the offender's index offence(s), conviction type, and parole sentence length.

\section{Data Analysis}

Data were provided by the New Zealand Department of Corrections in the form of individual records for each assessment and for each offender. SAS 9.1 was used to collate the data into one record per offender, as well as to clean the dataset. Lisrel 8.0 (Jöreskog \& Sörbom, 2004) was used for confirmatory factor analyses, while HLM 7.01 (Raudenbush \& Bryk, 2011) was used for growth modelling. SPSS 17.0 was used for all remaining analyses.

For analyses using the first assessment per offender, the third assessment period was selected to represent this baseline. The third assessment was chosen because it is still an initial time period but far enough along to allow raters to grow accustomed to the scaling and have sufficient information about the client to adequately score the DRAOR. For analyses using the last assessment per offender, the last assessment prior to recidivism or study end date was selected for each offender. For recidivists, the last assessment prior to reconviction during the first parole term was used. For nonrecidivists, the last assessment prior to the study end date (i.e., censoring) was used. 


\section{Empirical Litmus Test}

There is no gold standard or empirical litmus test established for the statistical analysis of the relationship between change data and recidivism in correctional research (Brown et al., 2009). Two common approaches were taken in the past that were consistent with early definitions of dynamic risk factors and two-wave research designs. The first involved creating artificial change variables (e.g., $-1=$ negative, $0=$ no change, $1=$ positive change) coded from existing data (e.g., Bonta, 1996; Hanson \& Harris, 2000; Motiuk 1999), but this approach loses valuable information for offenders who reoffend prior to reassessment (Brown, 2002) and does not capture the magnitude of change. The second approach employed hierarchal regression to examine whether re-test scores explained more variance in outcome than pre-test scores alone (e.g., Andrews \& Wormith, 1984; Campbell \& Kenny, 1999), but this also excludes individuals who reoffend prior to reassessment (Brown, 2002). In one of the first multi-wave research designs, Brown (2002) utilized three statistical analyses for the empirical litmus test for determining whether or not a dynamic risk factor is truly dynamic. These included standard within-subject change analyses, Receiver Operating Characteristic (ROC) analysis, and Cox regression survival analysis with time-invariant and time-dependent covariates. Quinsey, Jones, and colleagues (2006) also used within-subject analyses to assess whether forensic patients with institutional incidents demonstrated changes in their dynamic risk in the time leading up to the incident.

Based on previous methods of analyzing change data in relation to recidivism and the unique research design of the current study, three types of analyses will be performed specific to dynamic risk factors and recidivism. Similar to Quinsey, Jones, and 
colleagues (2006), novel statistical analyses will be utilized that are specifically intended for the type of longitudinal data collected. These analyses include ROC analysis, multilevel growth modelling, and Cox regression survival analysis. Multilevel growth modelling is employed in lieu of standard within-subject analyses given the advantages of this approach, to be discussed below.

Receiver Operating Characteristic analysis. Receiver Operating Characteristic (ROC) analysis, and Area Under the Curve (AUC) statistics in particular, were used to assess the ability of the DRAOR and its subscales to accurately forecast reconvictions. AUCs provide an assessment of discrimination, the extent to which recidivists are different from non-recidivists (Gail \& Pfeiffer, 2005). The AUC is the probability that a score on a measure drawn at random from one sample or population (e.g., scores of reconvicted offenders) is higher than that drawn at random from another sample or population (e.g., scores of offenders who are not reconvicted). Values range from .00 to 1.00 , with .50 being equivalent to chance and 1.00 being associated with $100 \%$ predictive accuracy. The advantages of AUCs are that they are independent of both the base rate of the outcome measure (e.g., reconvictions) and of the cut-off score selected to identify cases as probable to fail parole (Rice \& Harris, 1995). The AUC provides a standard for comparing the predictive accuracy of measures across correctional research, and has been recommended as the preferred measure of predictive accuracy (Mossman, 1994; Rice \& Harris, 2005; Swets, Dawes \& Monahan, 2000). The index is also ideal for comparing the performance of different instruments for the prediction of recidivism (Rice \& Harris, 1995). 
To statistically compare the predictive accuracy of dynamic and static models, the AUCs were compared using the Delong method for computing the standard error of the difference (Delong, Delong, \& Clarke-Pearson, 1988). This precise method of computing variances is one of the most common methods to test the extent to which risk scales differ in their level of discrimination (Babchishin, Hanson, \& Helmus, 2012). Given that it is suitable for even highly correlated AUC areas, Babchishin and colleagues (2012) recently used this method in a similar manner to test differences between risk scales.

Growth modelling. Growth modelling, a type of multilevel modelling, was used to assess whether offenders who were reconvicted showed changes in their dynamic risk levels and protective factors in the time leading up to the reconviction. Many traditional longitudinal approaches (e.g., repeated measures MANOVA) are unable to easily handle unbalanced data, missing data, or uneven time points. Unlike structured equation modelling (SEM), growth modelling can handle time-unstructured longitudinal designs in which participants are sampled at different intervals of time (Singer \& Willett, 2003). Growth modelling is flexible and efficient and allows for an examination of changes within an offender (e.g., dynamic risk, protective factors), while taking into account differences between offenders (e.g., static risk). This approach provides an understanding of average growth trends as well as variables that explain variability in growth.

Growth models are ideal for this longitudinal research design given that the same participants are assessed multiple times. Growth models provide an understanding of average growth trends across time in the outcome (i.e., dynamic risk factors), as well as 
an understanding of person variables that explain variability in growth (e.g., age, static risk). This analysis examines a level-1 model which describes how each offender changes over time, and a level-2 model which describes how these changes differ across offenders. This approach permits the modelling of intra-individual change, rather than inter-individual change that can be masked at the group level.

Cox regression survival analysis. Cox regression survival analysis was used to test the incremental validity of the total scores for the static and dynamic scales. This analysis examines whether the DRAOR predicts recidivism above and beyond the RoC*RoI. The dependent variable was time-at-risk (i.e., number of days between parole start and either recidivism date for recidivists, or study end date for non-recidivists). Each case was identified as either a failure (i.e., recidivist) or censored (i.e., still at risk at the end of follow-up).

Cox regression survival analysis was also used to assess the importance of the timing of assessments. A set of 12 regressions were performed to test the impact of monthly covariates on recidivism in the 12 months prior to recidivism for recidivists or censoring for non-recidivists. The monthly covariates consisted of average Stable Risk, Acute Risk, and Protective Factors for the given month preceding follow-up end. This analysis tests whether these offender-level covariates impact the likelihood of recidivism and time until recidivism. A comparison of effect sizes for each analysis determines whether there is a trend for later assessments to better predict recidivism than earlier assessments.

Cox regression analysis results in a hazard ratio or odds ratio, which is the predicted change in the hazard (i.e., the event of recidivism occurring) for a unit increase 
in the predictor (e.g., Stable Risk, Acute Risk, and Protective factors). The hazard function is an equation representing the instantaneous probability of recidivism occurring at any point in time, plotted over time. This is a semi-parametric analysis in that no assumption is made about the shape of hazard function, but assumptions are made about how covariates affect the hazard function.

\section{Organization}

The data analysis was conducted in six parts. Part 1 describes the sample and reports on methodological and statistical issues such as sample representativeness, missing data, and data screening. Part 2 examines the psychometric properties of the DRAOR, resulting from a series of exploratory and confirmatory factor analyses to refine and validate the DRAOR. Part 3 examines differences between offenders with and without reconvictions using between-offender analyses. Using Receiver Operating Characteristic (ROC) analysis, Part 4 explores the predictive accuracy of the DRAOR and the $\mathrm{RoC}^{*} \mathrm{RoI}$ as a measure of static risk. Part 5 examines change in dynamic risk over a period of assessment and reassessment using multilevel growth modelling. Lastly, Part 6 expands this analysis and adds Cox regression survival analysis to address the timing of DRAOR assessments. 


\section{Results}

\section{Part 1. Description of Sample}

Sample representativeness. The characteristics of the sample were compared to recent New Zealand release population cohorts to assess its representativeness, as displayed in Table 5. The 2009 Offender Volumes Report presents information about the offender population and analyzes how patterns in age, gender, and ethnicity have changed over the past few decades (New Zealand Department of Corrections, 2009). The gender distribution of the current study is similar to the data from $2008 / 2009$, although the percentage of females is slightly lower than recent figures. The Maori population is marginally higher, and there are fewer European and Pacific peoples than the comparison population. The distribution by age group is markedly similar between the study sample and the $2008 / 2009$ offender population.

In terms of the recidivism rate of $43.3 \%$, this is slightly higher than that found in a study of the reconviction and reimprisonment patterns for 5000 New Zealand offenders released from prison during a 12-month period from 2002 to 2003 (Nadesu, 2009). During the 60 month follow-up period used in the comparison study, $26 \%$ of the sample had reoffended, been reconvicted and returned to prison in the first year and $37 \%$ of the sample met these criteria in the second year. This data is restricted to convictions for new offences resulting in imprisonment, whereas the recidivism data for the study sample also includes reconvictions resulting in community-based sentences. As such, the slightly higher recidivism rate in the current study was to be expected event though not all offenders were in the community for a full two-year period following release. 
Table 5

Sample Representativeness: Study Sample (N = 3498) vs. 2008/2009 Offender Population $(N=4514)$

\begin{tabular}{|c|c|c|}
\hline Variable & $\begin{array}{c}\text { Sample } \\
\%(n)\end{array}$ & $\begin{array}{c}\text { 2008/2009 Offender } \\
\text { Population } \\
\%(n)\end{array}$ \\
\hline \multicolumn{3}{|l|}{ Gender } \\
\hline Male & 92.9 (3249) & 90.9 (4103) \\
\hline Female & $7.0(246)$ & $9.1(410)$ \\
\hline Unknown & $0.1(3)$ & $0.04(2)$ \\
\hline \multicolumn{3}{|l|}{ Ethnicity } \\
\hline Maori & $52.1(1824)$ & $50.0(2258)$ \\
\hline European & 37.3 (1308) & 38.7 (1746) \\
\hline Pacific Peoples & $7.5(261)$ & $8.8(398)$ \\
\hline Asian & $2.0(71)$ & $1.4(65)$ \\
\hline Other & $0.8(29)$ & $0.6(26)$ \\
\hline Unknown & $0.1(5)$ & $0.5(21)$ \\
\hline \multicolumn{3}{|l|}{ Age } \\
\hline $15-19$ & $2.5(87)$ & $5.1(229)$ \\
\hline $20-29$ & 36.8 (1287) & 35.4 (1599) \\
\hline $30-39$ & $28.0(980)$ & $28.0(1266)$ \\
\hline $40-49$ & $21.3(747)$ & $20.4(922)$ \\
\hline $50-59$ & $7.8(273)$ & $7.4(333)$ \\
\hline $60+$ & $3.5(121)$ & $3.6(163)$ \\
\hline Unknown & $0.1(4)$ & $0.1(3)$ \\
\hline
\end{tabular}

Note. Data for the 2008/2009 Offender Population is based on averages calculated from 12 snapshots from July 2008 to June 2009.

Statistical and methodological issues. Descriptive information for DRAOR items, subscales, and the $\mathrm{RoC}^{*} \mathrm{RoI}$ is displayed in Table 6 . All variables were examined for data entry accuracy and the presence of missing values. There were five out-of range data entry errors in the dates of the DRAOR assessments for five different offenders. These assessments were excluded because there was not enough information to determine the correct date and their position in the chronology of DRAOR assessments for their respective offenders. There were 18 assessments completed prior to the April 1, 2010 
study start date before the DRAOR was implemented for all parole contacts. All of these were either the first or second assessment for each of the offenders. Given that the third DRAOR assessment was used as a baseline assessment, they were included in the sample as their baseline assessment occurred after April 1, 2010. With the exception of the 196 offenders released on parole for which no data was available regarding their static risk, DRAOR assessments or recidivism, there was no other missing data in the variables of interest.

Table 6

Descriptive Information of Risk Measures

\begin{tabular}{lccc}
\hline \multicolumn{1}{c}{ Variable } & Range & $M$ & $S D$ \\
\hline RoC*RoI & $.00-.97$ & .47 & .24 \\
Acute Total & $0-14$ & 4.62 & 2.45 \\
Substance abuse & $0-2$ & 0.47 & 0.62 \\
Anger/hostility & $0-2$ & 0.28 & 0.52 \\
Opportunity/Access to victims & $0-2$ & 0.82 & 0.60 \\
Negative mood & $0-2$ & 0.33 & 0.55 \\
Interpersonal relationships & $0-2$ & 1.35 & 0.81 \\
Employment & $0-2$ & 0.98 & 0.67 \\
Living situation & $0-2$ & 0.38 & 0.58 \\
& & & \\
Protective Total & $0-12$ & 7.24 & 2.66 \\
Responsive to advice & $0-2$ & 1.26 & 0.60 \\
Prosocial identity & $0-2$ & 1.12 & 0.60 \\
High expectations & $0-2$ & 1.31 & 0.62 \\
Costs/benefits & $0-2$ & 1.22 & 0.62 \\
Social support & $0-2$ & 1.33 & 0.61 \\
Social control & $0-2$ & 1.00 & 0.55 \\
Stable Total & & & \\
Peer associations & $0-12$ & 6.62 & 3.79 \\
Attitudes toward authority & $0-2$ & 0.96 & 0.66 \\
Impulse control & $0-2$ & 0.65 & 0.70 \\
Problem solving & $0-2$ & 1.03 & 0.67 \\
Sense of entitlement & $0-2$ & 0.98 & 0.65 \\
Attachment with others & $0-2$ & 0.95 & 0.71 \\
\hline
\end{tabular}


Variables were examined for linearity, homoscedasticity, normality, univariate and multivariate outliers, and multicollinearity. Assumptions were tested at the level of DRAOR subscales, as opposed to individual items, as these were included in the analyses. Pairwise plots for each of the variables suggested linear relationships between each combination of variables. The assumption of homoscedasticity also appears to be supported with roughly the same variability in scores for each variable at all values of the other variables.

Kolmogorov-Sminov's test of normality was performed on each of the variables to test the hypothesis that the distribution of each variable is not significantly different from a normal distribution. Not surprisingly given the large sample size, the test was significant $(p<.001)$ for all variables, suggesting that the distributions depart from normality. This is to be expected in large datasets even when departures from normality are slight; therefore it is more prudent to inspect the shape of the distribution instead of using formal inference tests. An examination of the data (i.e., histograms, probability plots, kurtosis and skewness values) suggests the departures from normality are not grave. Using a conventional but conservative alpha level of .001 , nearly all of the standardized scores for skewness and kurtosis were within the critical values of 3.29 (two-tailed test). The Acute Risk subscale had 208 assessments exceeding the critical value, with $.002 \%$ of the data having a standardized score of 3.42 and $.0004 \%$ of the data having a standardized score of 3.83. However, none of the cases appeared 'unattached' from the rest of the distribution and all of the values could have reasonably come from the population, given the individual differences in offenders. Given the proximity to the 
conservative critical point and in the interest of clear interpretation, no transformations were made to this variable. There were no other univariate outliers in the data.

Mahalanobis' distance was used to identify multivariate outliers. There were 214 cases which exceeded the critical value of $18.467(d f=4, \alpha=.001)$. A linear regression was performed to identify the variables causing the multivariate outliers. The outliers differed from the rest of the cases on all four variables entered into the regression. The outlying cases had higher scores on Acute Risk, Stable Risk, Protective Factors, and the RoC*RoI. Half of the outliers $(n=30)$ were found in multiple assessments within offenders, while the remaining 31 outliers occurred once per individual offender. One of the very purposes of this study is to locate peaks in DRAOR assessments which may indicate that recidivism is imminent. As such, to avoid limiting the generalizability of the study and to maintain assessment sequences, the cases were not deleted from the study.

Lastly, all variables were evaluated for multicollinearity and singularity using bivariate correlations and tolerance values. There were no perfect or near perfect correlations among the variables, the largest correlation being between Stable Risk and Protective Factors (Pearson $r=-.71, p<.001)$. Inter-correlations among subscales and the $\mathrm{RoC}^{*}$ RoI are displayed in Table 7 and inter-correlations among DRAOR items are displayed in Appendix B.

Table 7

Inter-Correlations Among Risk Measures $(N=3365)$

\begin{tabular}{lccc}
\hline & Stable Risk & Acute Risk & Protective Factor \\
\hline RoC*RoI & $.35 * *$ & $.30 * * *$ & $-.31 * * *$ \\
Stable Risk & & $.61 * * *$ & $-.71^{* * *}$ \\
Acute Risk & & & $-.59 * * *$ \\
\hline
\end{tabular}

Note. Correlations were calculated using the last DRAOR assessment prior to recidivism or study end. $* * * p<.001$. 
The final assumption tested is the proportionality of hazards and is specific to Cox regression survival analysis. This test assumes that the shape of the survival function is the same for all cases and all groups over time. Violation of this assumption indicates an interaction between groups and time in survival rates, or between covariates and time. This was tested by examining the log minus log plots of the hazard functions for each covariate median-split into a categorical variable. The assumption was not violated as all of the lines were parallel and did not cross one other.

Summary: Part 1. The study sample is comparable to recent offender populations in New Zealand. There are no detrimental statistical or methodological issues in the data that will affect the conclusions of the subsequent analyses.

\section{Part 2. Refining and Validating the DRAOR}

Hypothesis 1. The DRAOR will demonstrate good psychometric properties (e.g., factor structure, Cronbach's $\alpha$, item-total correlations).

Given the substantial sample size and the longitudinal collection of DRAOR assessments, the factor structure and psychometrics of the DRAOR were examined across time periods. An Exploratory Factor Analysis (EFA) was first performed on the initial DRAOR assessment to understand its underlying structure. EFA is used to describe and summarize data by grouping together correlated variables (Tabachnick \& Fidell, 2007). It is typically used in the early stages of research, and the variables entered may or may not have been chosen with potential underlying processes in mind (i.e., a priori notions regarding the nature and number of factors being sought; Tabachnick \& Fidell, 2007). ${ }^{7}$ It is especially reassuring, however, when items group together as suspected because the

\footnotetext{
${ }^{7}$ EFA is employed in the present research rather than principal components analysis (PCA) since PCA is a purely empirical test wherein theory does not drive the decision making process. EFA is more appropriate than PCA when there are a priori notions regarding the nature and number of factors being sought.
} 
analysis has not been instructed to identify a specific pattern (DeVellis, 2003). The EFA resulted in two possible models in addition to the original DRAOR, whose psychometric properties were explored. The three models were tested at the middle and final assessment periods using Confirmatory Factor Analysis (CFA). CFA is typically used in the advanced stages of the research process when testing a theory about latent processes (Tabachnick \& Fidell, 2007). This analysis answers a deeper question than just the factor structure and psychometrics of the scale, but also confirms the structure's stability across time. The elegance of this approach is that the same sample but new data is used across time periods, so the CFAs address the issue of factor structure of the data without differences in participants from sample to sample interfering with the ability to make conclusions about data structure. The CFAs were tested using Lisrel 8.0 (Jöreskog \& Sörbom, 2004), which is appropriate for ordinal data.

Exploratory factor analysis. An exploratory factor analysis was performed using the baseline assessment to determine if the 19 DRAOR item scores clustered as predicted on the three subscales of Stable Risk, Acute Risk, and Protective Factors. ${ }^{8}$ Participants required a minimum of three assessments to be included in the analysis as the third assessment was used as the baseline. The sample size of 3427 was ideal for generating reliable and replicable factors. Despite most communalities being lower than .5 , the present sample size is able to produce reliable factors (MacCallum, Widaman, Zhang, \& Hong, 1999). Kaiser-Meyer Olkin's measure of sampling adequacy (KMO =

\footnotetext{
${ }^{8}$ Ideally, factor analysis should be conducted on continuous variables as opposed to dichotomous or categorical variables as is the case in the current study. Categorical variables result in relatively little variability and the correlations between items may be small and unreliable. However, these limitations are minimized by using a large sample size and a large subject-to-variable ratio (Kline, 1994).
} 
.91) suggests the present data are adequate for conducting factor analysis. The ratio of subjects to variables is highly adequate at a ratio of 180:1.

A Pearson correlation matrix was examined to determine the factorability of the data and to check for singularity and multicollinearity. The matrix consisting of 19 crosscorrelated items yielded 342 correlations. Of these, 66 were .30 or greater. The highest correlation was .63. The determinant of the $\mathbf{R}$ matrix (.003) does not suggest issues of multicollinearity in the data. Bartlett's test of sphericity was significant $\left(\chi^{2}(d f=171)=\right.$ 19811.29, $p<.001$ ) which indicates that the $\mathbf{R}$ matrix is factorable. Given the results of this test and the number of correlations greater than .30 , factor analysis is justifiable.

An initial solution was created using principal factors analysis with direct quartimin rotation $(\delta=0)$. The principal factors extraction method was used to analyze common variance while eliminating unique and error variance from variables. The direct quartimin rotation method was chosen to allow factors to correlate while maximizing the simplicity of the factors (Tabachnick \& Fidell, 2007). ${ }^{9}$ The solution identified four factors with eigenvalues greater than 1.0. Kaiser's criterion (i.e., retaining eigenvalues greater than 1.0) is generally accurate when there are less than 30 variables and communalities after extraction are greater than .7 or when the sample size exceeds 250 and the average communality is greater than .6 (Field, 2005). Although there are 19 variables and a large sample size, there are no communalities greater than .7 and the average communality is only .40. Based on these grounds, Kaiser's criterion may not be accurate. An examination of the scree plot's point of inflexion also suggests three to four components should be retained. Lastly, when eigenvalues derived from a randomly

\footnotetext{
${ }^{9}$ Orthogonal rotation methods (e.g., varimax) should only be used when there is a strong theoretical reason for assuming factors to be orthogonal (Kline, 1993). Oblique rotation methods (e.g., direct quartimin) are ideal as they do not exclude the possibility of an orthogonal structure being detected (Kline, 1994).
} 
generated dataset with equivalent features (i.e., parallel analysis; number of cases $=3427$; number of variables $=19 ; 100$ replicated datasets were employed) were compared to observed eigenvalues, the results suggested the first three factors should be retained. Parallel analysis has been shown to be one of the most reliable strategies for component retention (Zwick \& Velicer, 1986), therefore the first three factors were retained. The three identified factors explain $38.2 \%$ of the variance. Within the three factors, 17 of the 19 items were represented using a loading criterion of .32 (Tabachnick \& Fidell, 2007). Six of these were complex variables that loaded on more than one factor. Cross-loaded items were assigned to multiple factors if they exceed the loading criterion of .32. As can be seen in the structure and pattern matrices in Tables C.1 and C.2 in Appendix C, Factor 1 represents Mostly Acute items. All of the DRAOR Acute subscale items loaded onto this factor, with the exception of 'Interpersonal Relationships' and 'Employment' which did not load onto any factor. These two items were removed to create a more parsimonious model. In addition, the following Stable subscale items loaded onto the Mostly Acute factor: 'Attitudes toward Authority,' 'Sense of Entitlement,' and 'Attachment with Others.' Factor 2 was identified as Mostly Stable given that all of the DRAOR Stable subscale items loaded onto this factor, as well as the following Acute items: 'Opportunity/Access to Victims,' 'Anger/hostility,' and 'Substance Abuse.' Lastly, the six DRAOR Protective subscale items loaded exclusively onto Factor 3, Protective. The structure matrix does not ignore shared variance, while the pattern matrix contains information about the unique contribution of a variable to a factor. An examination of the structure matrix suggests most of the stable and acute items load highly onto more than one factor. This is not surprising given the strong inter- 
correlations among the factors. The Mostly Stable and Mostly Acute factors have a medium correlation of .49. The Protective Factor has medium to large negative correlations with Mostly Stable $(r=-.39)$ and Mostly Acute $(r=-.66)$. The total variance explained by this model using principal factors analysis was $38.2 \%$, with most of the variance accounted for by Mostly Acute (29.9\%).

The stability of the present solution was examined using the generalized least squares extraction method. This method was chosen because it weights items that share substantial variance with other variables. As displayed in the structure and pattern matrices in Tables C.3 and C.4 in Appendix C, there were some differences to the factor structure derived from principal factors analysis in terms of the factor loadings. First, a similar amount of variance was explained by this model (38.6\%), but most of the variance was accounted for by the Stable Factor 1 (30.0\%). The factor loadings were identical to those of the Mostly Stable factor from the principal factors analysis, with the exception of the 'Substance Abuse' Acute item which did not load. The factor loadings of Factor 2 were identical to the Protective from the first model. Factor 3 was identified as Mixed Stable/Acute as five items from each of the DRAOR Stable and Acute subscales loaded. As with the first model, 'Interpersonal Relationships' and 'Employment' did not load onto any factor. The Stable and Mixed Stable/Acute factors have a medium correlation of .53, which is unsurprising given the overlap in items. The Protective Factor has medium to large negative correlations with Stable $(r=-.64)$ and Mixed Stable/Acute $(r=-.45)$. 
Psychometric properties. Given the instability of the factor structure as evidenced by differences in the factor loadings depending on the method of extraction used, the psychometric properties of each were examined. Model \#1 refers to the original DRAOR subscales. Model \#2 refers to the factor structure generated using principal factors analysis, while Model \#3 is based on the results of the generalized least squares extraction method. The internal consistency of the identified factors using Cronbach's alpha is displayed in Table 8 for Model \#1, Table 9 for Model \#2, and Table 10 for Model \#3. Each of the factors representing the three models demonstrated acceptable internal consistency; with the exception of the original Acute DRAOR subscale (Model \#1) which has poor internal consistency. The highest internal consistency is demonstrated by Model \#3 which has pure Protective and Stable factors, as well as a Mixed Stable/Acute factor.

Item-total correlations and Cronbach's alpha if items are deleted are displayed in Appendix D for Model \#1, Appendix E for Model \#2, and Appendix F for Model \#3. For the original DRAOR subscales (Model \#1), internal consistency for the Acute subscale would increase from .62 to .63 if 'Interpersonal Relationships' was removed. Corrected item-total correlations are also low for some of the items on the Acute subscale, indicating that not all items correlate with the overall score from the scale. Cronbach's alpha would not be increased by deleting any of the other items on the remaining factors. For Models \#2 and \#3, internal consistency would not be improved by deleting any of the items of any of the factors. However, internal consistency would remain the same by deleting Acute items from the Stable subscale in Model \#3. Cronbach's alpha for the Stable subscale would remain the same if the Acute items 'Anger/hostility' and 
'Opportunity/access to victims' were deleted. In the interest of parsimony and conceptual clarity, the decision was made to delete these items and reduce Model \#3 to factors consisting of purely Protective, purely Stable, and a Mixed Stable/Acute factor.

Table 8

Internal Consistency and Descriptive Statistics for Model \#1 DRAOR Subscales

\begin{tabular}{lccccc}
\hline DRAOR Subscale & $\alpha$ & $M$ & $S D$ & Range & $\begin{array}{c}\text { \# of } \\
\text { items }\end{array}$ \\
\hline Stable & .81 & 6.08 & 2.72 & $0-12$ & 6 \\
Acute & .62 & 5.45 & 2.40 & $0-12$ & 7 \\
Protective & .84 & 6.40 & 2.52 & $0-12$ & 6 \\
\hline
\end{tabular}

Table 9

Internal Consistency and Descriptive Statistics for Model \#2 DRAOR Factors

\begin{tabular}{lccccc}
\hline DRAOR Factor & $\alpha$ & $M$ & $S D$ & Range & $\begin{array}{c}\text { \# of } \\
\text { items }\end{array}$ \\
\hline Mostly Stable & .81 & 8.00 & 3.56 & $0-18$ & 9 \\
Mostly Acute & .75 & 5.47 & 3.02 & $0-16$ & 8 \\
Protective & .84 & 6.40 & 2.52 & $0-12$ & 6 \\
\hline
\end{tabular}

Table 10

Internal Consistency and Descriptive Statistics for Model \#3 DRAOR Factors

\begin{tabular}{lccccc}
\hline DRAOR Factor & $\alpha$ & $M$ & $S D$ & Range & $\begin{array}{c}\text { \# of } \\
\text { items }\end{array}$ \\
\hline Stable & .81 & 7.35 & 3.26 & $0-16$ & 8 \\
Mixed Stable/Acute & .80 & 7.77 & 3.72 & $0-20$ & 10 \\
Protective & .84 & 6.40 & 2.52 & $0-12$ & 6 \\
\hline
\end{tabular}


Confirmatory factor analysis. Confirmatory factor analysis was used to examine whether the factors found during the exploration stage were confirmed with the same sample of data using different time periods, as well to compare the fit of competing hypothesized models. Measurements were collected on the middle $(N=3331)$ and last DRAOR assessment $(N=3372)$, which varied by participant. Participants required a minimum of five assessments to be included in the middle assessment sample (i.e., baseline third assessment plus two additional assessments). Likewise, participants required four assessments to be included in the last assessment sample (i.e., baseline third assessment plus one additional assessment). The last assessment refers to the last assessment prior to the first recidivism date during the study period for recidivists or the study end date for non-recidivists.

Model testing process. Weston and Gore (2006) outline five steps in structural equation modelling that are necessary for complete model testing: model specification, identification, estimation, evaluation, and modification.

Model specification. The relationships between the latent variables and the corresponding observed variables are displayed in Figures 1, 2, and 3 for Models \#1 (Original DRAOR subscales), \#2 (Principal Factors Analysis), and \#3 (Generalized Least Squares), respectively. Model \#1 consisted of 19 observed variables (i.e., each item on the DRAOR) and three latent variables (i.e., factors) relating to dynamic risk and protective factors. These factors are Stable Risk, Acute Risk, and Protective Factors. Models \#2 and \#3 consisted of 17 observed variables and three latent variables. For Model \#2, these factors are Mostly Stable Risk, Mostly Acute Risk, and Protective Factors. For Model \#3, these factors are Stable Risk, Mixed Stable/Acute Risk, and 
Protective Factors. For each of the models, the factors were specified to be intercorrelated with each other, with three inter-correlations total. The path between the observed variables and the associated latent variables were estimated, while all other paths were fixed. In other words, all observed variables had paths estimated to the a priori hypothesized latent variable. There are 19 error variances for Model \#1 and 17 error variances for Models \#2 and \#3, one for each observed variable.

Model identification. To test the model, there must be more values in the sample covariance matrix than the number of free parameters being estimated. For Model \#1, the DRAOR has 19 observed variables, indicating that the model has 190 known elements in the sample covariance matrix. ${ }^{10}$ There are 41 unknown parameters that must be estimated (i.e., 19 error variances, 3 inter-correlations among factors, and 19 factor loadings to be estimated). Subtracting 41 unknown parameters from 190 elements leaves 149 degrees of freedom, indicating the model is over-identified (i.e., a value for each path may be found in many ways). For Models \#2 and \#3, there are 153 known elements (i.e., 17 observed variables) and 37 free parameters being estimated (i.e., 17 error variances, 3 inter-correlations among factors, and 17 factor loadings to be estimated). Both of these models are also over-identified with 116 degrees of freedom, meeting the order condition required to test the model.

Model estimation. Weighted Least Squares estimation was used since the data was ordinal. The specified confirmatory factor analyses were conducted to determine whether the observed variables loaded onto latent variables, as hypothesized.

\footnotetext{
${ }^{10}$ Determining the identification measurement model requires determining the number of degrees of freedom by calculating the number of known elements in the model using the equation $p(p+1) / 2$, where $p$ is the number of observed variables.
} 
Figure 1. Confirmatory factor analysis of Model \#1.

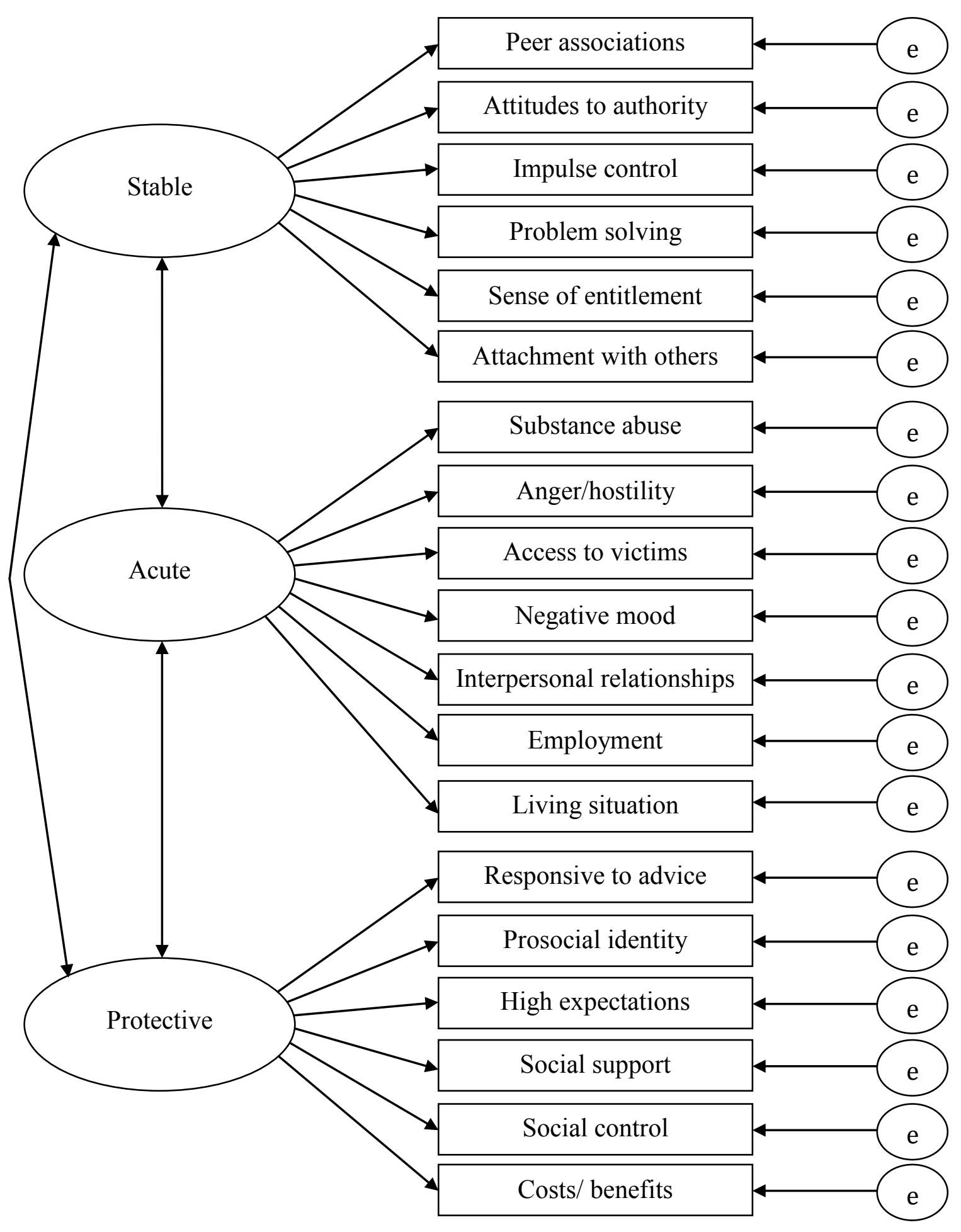


Figure 2. Confirmatory factor analysis of Model \#2.

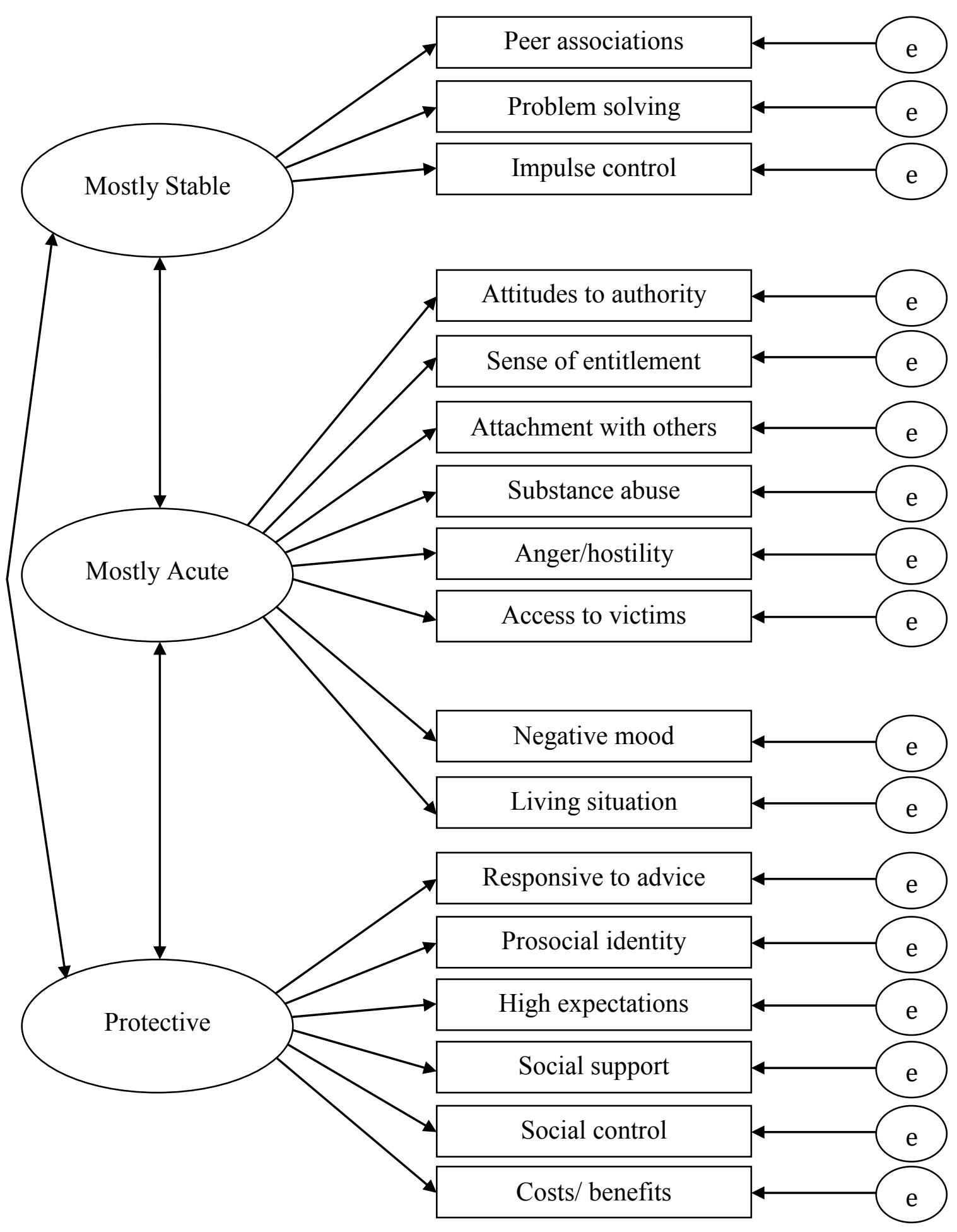


Figure 3. Confirmatory factor analysis of Model \#3.

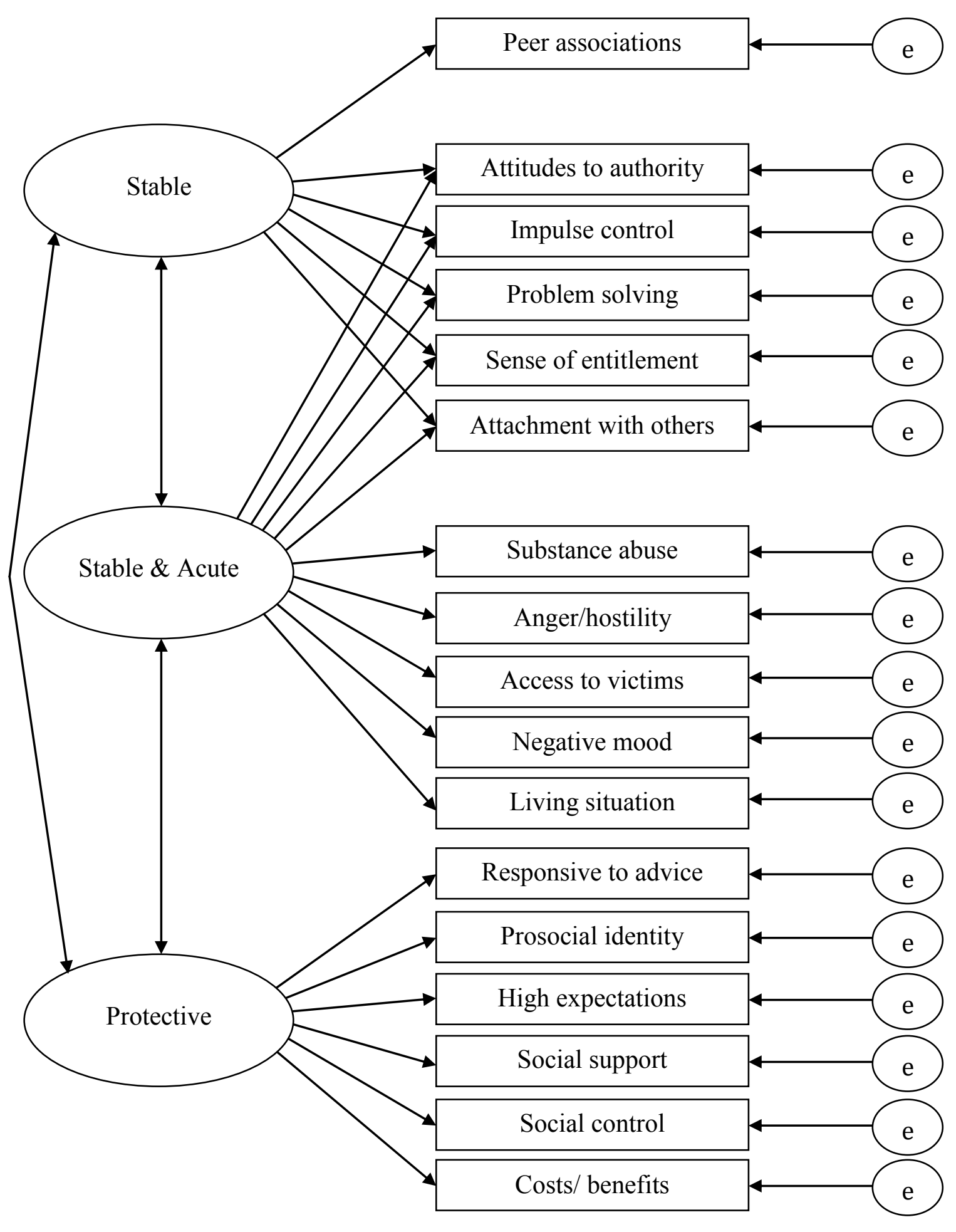


Model evaluation. A combination of fit indices was used to determine the adequacy of the model fit. Although a non-significant Chi-square goodness of fit test indicates that the model adequately accounts for the data, the test is highly sensitive to the size of the sample. This means that tests involving large samples will generally lead to a rejection of the null hypothesis, even when the factor model is appropriate. This value is reported along with the Chi-square/degrees of freedom ratio, but other tests of model adequacy that are less sensitive to sample size were used to measure goodness of fit. Based on the type of data (i.e., ordinal), the following criteria were used in the study to determine the adequacy of model fit: (a) Goodness of Fit Index (GFI) greater than .90, (b) Comparative Fit Index (CFI) greater than .90, and (c) Root Mean Square Error of Approximation (RMSEA) less than .06. The GFI measures the amount of variance and covariance accounted for by the model while the CFI compares the existing model fit with a null uncorrelated model. The RMSEA measures the size of the residuals in the model and the discrepancy between observed and estimated covariance per degrees of freedom. Lastly, the Akaike Information Criterion (AIC) fit index will be used to compare the three non-nested models to determine which best fit the data. A smaller AIC is indicative of a model that is more likely to replicate, has fewer parameters, and fits better.

Model modification. If the initial model did not have an adequate fit based on the criteria selected, modification indices were used to determine changes needed to improve the model fit. These changes are based on how the data fit the model, but it is important to only make changes based on whether those changes would be supported by theory. If any changes are made, the model must be re-evaluated. 
Model testing at middle assessment. As can be seen in Table 11, the Chi-square is rather large for all three models suggesting that the models do not produce a small difference between observed and expected matrices. In addition, the Chi-square value for each model is more than double its degrees of freedom (i.e., the $\chi^{2} / d f$ ratio exceeds the recommended 2; Byrne, 1989). These findings were expected given the sensitivity of these tests to large sample sizes. However, the GFI for all three models indicates good fit, supported by the CFI values. The unexplained variance or residual is acceptable for all three models, as evidenced by the RMSEA values. Given that all of the criteria used to determine model fit were met or exceeded, no modifications were necessary. While all three hypothesized models were a good fit to the observed data, the AIC value for Model \#3 was lower than Models \#1 and \#2, indicating that the model with Stable, mixed Stable and Acute, and Protective Factors best fit the data.

Table 11

Goodness-of-Fit Indices for Three Models using the Middle DRAOR Assessment

\begin{tabular}{lccc}
\hline & \multicolumn{3}{c}{ Model } \\
\cline { 2 - 4 }$\chi^{2}$ & 1 & 2 & 3 \\
$d f$ & 1471.8 & 1334.7 & 1301.2 \\
$p$ & 149 & 110 & 111 \\
$\chi^{2} / d f$ & $<.0001$ & $<.0001$ & $<.0001$ \\
GFI & 9.88 & 12.13 & 11.72 \\
CFI & .98 & .98 & .98 \\
RMSEA & .93 & .91 & .92 \\
AIC & .05 & .06 & .06 \\
\hline
\end{tabular}


Model testing at last assessment. The fit indices displayed in Table 12 suggest similar results for the three models using the last DRAOR assessment. The Chi-square values are large and the probabilities are unacceptable, due to the sensitivity of this test. Based on the goodness-of-fit criteria selected, all three models demonstrate good fit and acceptable unexplained variance. No post-hoc modifications were indicated from the analysis to any of the models based on the good-fit indices. However, the AIC value for Model \#2 was lower than Models \#1 and \#3, indicating that the model with Mostly Stable, Mostly Acute, and Protective Factors best fit the data for the last DRAOR assessment.

Table 12

Goodness-of-Fit Indices for Three Models using the Last DRAOR Assessment

\begin{tabular}{lccc}
\hline & \multicolumn{3}{c}{ Model } \\
\cline { 2 - 4 }$\chi^{2}$ & 1 & 2 & 3 \\
$d f$ & 1367.1 & 970.2 & 1214.7 \\
$p$ & 149 & 110 & 111 \\
$\chi^{2} / d f$ & $<.0001$ & $<.0001$ & $<.0001$ \\
GFI & 9.18 & 8.82 & 10.94 \\
CFI & .98 & .99 & .98 \\
RMSEA & .94 & .95 & .93 \\
AIC & .05 & .05 & .05 \\
\hline
\end{tabular}

Summary: Part 2. Refining and validating the DRAOR was a multi-stage process spanning across time periods of assessment. In addition to the original DRAOR subscales (Model \#1), two prospective models resulted from an Exploratory Factor 
Analysis using the initial DRAOR assessment scores. Model \#2 was the result of principal factors analysis and consisted of three factors: Mostly Stable, Mostly Acute, and Protective Factors. Model \#3 was the result of generalized least squares method and consisted of three factors: Stable, Mixed Stable/Acute, and Protective Factors. The stability of these models was tested using Confirmatory Factor Analysis of the middle and final DRAOR assessment scores. While all three models demonstrated acceptable fit across time periods, Model \#3 best fit the data using the middle assessment and Model \#2 demonstrated a better fit using the final assessment. Model \#3 was used in the subsequent analyses as it is both theoretically and practically more appealing than Model \#2. As a comparison, the original DRAOR subscales (Model \#1) was also tested in the remaining analyses. The purpose of this comparison is to determine whether the factor structure obtained using factor analysis methods makes a conceptual contribution and improves the practical utility of the DRAOR above its original subscales.

In addition to the original DRAOR subscale scores, factor scores were computed based on Model \#3. The factor scores provide a single indicator on each of the three factors for every participant. Factor scores were estimated by summing scores on variables that loaded highly on each factor, taking into account decisions that were made to drop variables based on an examination of their psychometric properties. All of the items have roughly equal standard deviations; therefore variables with differing standard deviations will not have markedly different contributions to the factor scores. This simple technique was deemed to be adequate in the interest of practical utility. One of the goals of this study is to refine and validate the DRAOR, and potentially create a second version of the assessment tool. Accordingly, probation officers require a simple 
method of summing items that is easy to use and understand to assist in their case management and supervision.

Given that the factor analyses were calculated using only the initial, middle, and last DRAOR assessments, the factor scores were calculated manually for the entire sample of DRAOR assessments. Only the Mixed Stable/Acute factor score was calculated as the Stable and Protective factor scores are identical to the original Stable and Protective subscale scores. For the Stable and Mixed Stable/Acute factors, higher factor scores correspond to a greater risk. For the Protective Factor, higher scores correspond to a greater protective buffer against reoffending. A Revised Total DRAOR score was calculated by summing all of the Stable and Acute items that loaded onto the factor solution and subtracting the Protective items (i.e., essentially removing Interpersonal Relationships and Employment from the original total score).

\section{Part 3. Differentiation of Recidivists and Non-Recidivists}

Hypothesis 2. Offenders with a reconviction will have higher stable and acute dynamic risk, lower protective factors, and higher DRAOR total scores.

Between-offender analyses were conducted to examine differences between offenders who had reconvictions and those who did not. ${ }^{11}$ The first stage of the analyses included all offenders, followed by a focus only on Maori offenders. Comparisons were first performed using all reconvictions, and then focusing on criminal reconvictions only. The two groups (recidivists versus non-recidivists) were compared using independent samples $t$-tests on static risk (i.e., RoC*RoI), overall dynamic risk (i.e., DRAOR total

\footnotetext{
${ }^{11}$ While this analysis provided some of the same information as the AUCs in Part 4, it does so in a manner that some readers may find more accessible. The between-offender analyses also set the stage for Part 4 by providing the rationale for using the last DRAOR assessment to establish predictive accuracy.
} 
score), as well as subscale and factor scores. These comparisons were made at the initial and final DRAOR assessment.

Due to the influence of a large sample size in finding significant results, a decision rule was established in the calculation of effect sizes. In the analyses of group differences, only small or larger effect sizes (Cohen's $\mathbf{d} \geq 0.20$ ) were considered indicative of a meaningful difference. Similarly sized effects are commonly found and considered meaningful in Forensic Psychology and offender populations in particular (Nunes \& Cortoni, 2006). For instance, Gendreau, Little, and Goggin (1996) found small to medium effect sizes in their meta-analysis for many predictors of criminal recidivism.

Overall sample. As demonstrated in Table 13, offenders who incurred a reconviction during the study period had higher levels of static risk (i.e., RoC*RoI scores). The differences between groups in terms of overall DRAOR scores, subscale scores, and factor scores were as expected at each of the time periods. Offenders with a reconviction had significantly higher levels of stable and acute dynamic risk, lower levels of Protective Factors, and higher scores on the DRAOR overall in comparison to offenders who were not reconvicted. The effect sizes were comparable for DRAOR subscale scores and factor scores. The differences between groups became even more apparent when comparing scores at the initial assessment to the last assessment either prior to recidivism or the study end period. The difference between means increased from medium effect sizes at the initial assessment to large effect sizes at the last assessment. When examining the means from the initial to last assessment for both groups, Stable and Acute Risk appears to decrease and Protective Factors increase. 
Table 13

Comparison of Recidivists versus Non-recidivists for Overall Sample

\begin{tabular}{|c|c|c|c|c|c|}
\hline Variable & $\begin{array}{l}\text { Recidivist } \\
M(S D)\end{array}$ & $\begin{array}{c}\text { Non-recidivist } \\
M(S D)\end{array}$ & $t$ & $d f$ & Cohen's d \\
\hline RoC*RoI & $.61(.19)$ & $.41(.25)$ & $-25.79 * * *$ & 3418 & .90 \\
\hline \multicolumn{6}{|c|}{ DRAOR Subscales } \\
\hline \multicolumn{6}{|l|}{ Original Total } \\
\hline Initial & $7.00(6.56)$ & $3.69(5.94)$ & $-15.46 * * *$ & 3425 & .53 \\
\hline Last & $5.52(7.34)$ & $.02(6.56)$ & $-22.63 * * *$ & 3370 & .79 \\
\hline \multicolumn{6}{|l|}{ Stable } \\
\hline Initial & $6.72(2.71)$ & $5.58(2.62)$ & $-12.46 * * *$ & 3425 & .43 \\
\hline Last & $6.25(2.94)$ & $4.48(2.84)$ & $-17.58 * * *$ & 3370 & .61 \\
\hline \multicolumn{6}{|l|}{ Acute } \\
\hline Initial & $6.15(2.47)$ & $4.92(2.20)$ & $-15.38 * * *$ & 3425 & .53 \\
\hline Last & $5.62(2.72)$ & $3.58(2.23)$ & $-23.91 * * *$ & 3370 & .82 \\
\hline \multicolumn{6}{|l|}{ Protective } \\
\hline Initial & $5.87(2.60)$ & $6.81(2.38)$ & $11.03 * * *$ & 3425 & -.38 \\
\hline Last & $6.35(2.86)$ & $8.04(2.61)$ & $17.94 * * *$ & 3370 & -.62 \\
\hline
\end{tabular}

DRAOR Factors

Revised Total $\begin{array}{llllll}\text { Initial } & 4.10(6.16) & 1.18(5.51) & -14.61 * * * & 3425 & .50\end{array}$ $\begin{array}{llllll}\text { Last } & 2.89(6.78) & -1.87(5.96) & -20.54 * * * & 3370 & .75\end{array}$

Stable/Acute

$\begin{array}{llllll}\text { Initial } & 8.73(3.84) & 7.02(3.44) & -13.71 * * * & 3425 & .47\end{array}$

$\begin{array}{llllll}\text { Last } & 8.06(4.14) & 5.36(3.47) & -20.54 * * * & 3370 & .71\end{array}$

Note. At the initial assessment, the sample size was 1497 for recidivists and 1930 for non-recidivists. At the last assessment, the sample size was 1483 for recidivists and 1889 for non-recidivists. The RoC*RoI analyses were based on 1493 recidivists and 1927 non-recidivists.

$* * * p<.001$.

These patterns were also observed when examining criminal reconvictions only (see Table 14). While impressive in their own regard, the effect sizes were smaller for criminal reconvictions than any reconvictions. This suggests that the DRAOR may be better at differentiating between recidivists and desisters for technical violations and general recidivism, rather than the more serious criminal convictions. 
Table 14

Comparison of Criminal Recidivists versus Non-recidivists for Overall Sample

\begin{tabular}{|c|c|c|c|c|c|}
\hline Variable & $\begin{array}{l}\text { Recidivist } \\
M(S D)\end{array}$ & $\begin{array}{c}\text { Non-recidivist } \\
M(S D)\end{array}$ & $t$ & $d f$ & Cohen's d \\
\hline RoC*RoI & $.64(.17)$ & $.44(.25)$ & $-22.72 * * *$ & 3418 & .94 \\
\hline \multicolumn{6}{|c|}{ DRAOR Subscales } \\
\hline \multicolumn{6}{|c|}{ Original Total } \\
\hline Initial & $7.01(6.74)$ & $4.36(6.13)$ & $-11.18^{* * *}$ & 3425 & .41 \\
\hline Last & $5.26(7.44)$ & $1.24(7.10)$ & $-14.79 * * *$ & 3370 & .55 \\
\hline \multicolumn{6}{|l|}{ Stable } \\
\hline Initial & $6.68(2.74)$ & $5.83(2.67)$ & $-8.44 * * *$ & 3425 & .31 \\
\hline Last & $6.13(2.96)$ & $4.89(2.97)$ & $-11.07 * * *$ & 3370 & .42 \\
\hline \multicolumn{6}{|l|}{ Acute } \\
\hline Initial & $6.23(2.53)$ & $5.13(2.27)$ & $-12.39 * * *$ & 3425 & .46 \\
\hline Last & $5.60(2.79)$ & $4.01(2.45)$ & $-16.47 * * *$ & 3370 & .61 \\
\hline \multicolumn{6}{|l|}{ Protective } \\
\hline Initial & $5.90(2.62)$ & $6.60(2.45)$ & $7.53 * * *$ & 3425 & -.28 \\
\hline Last & $6.46(2.86)$ & $7.65(2.77)$ & $11.30 * * *$ & 3370 & -.42 \\
\hline \multicolumn{6}{|l|}{ DRAOR Factors } \\
\hline \multicolumn{6}{|l|}{ Revised Total } \\
\hline Initial & $4.08(6.31)$ & $1.78(5.71)$ & $-10.41 * * *$ & 3425 & .38 \\
\hline Last & $2.65(6.86)$ & $-.80(6.45)$ & $-13.90 * * *$ & 3370 & .52 \\
\hline \multicolumn{6}{|l|}{ Stable/Acute } \\
\hline Initial & $8.74(3.93)$ & $7.37(3.55)$ & $-9.96 * * *$ & 3425 & .37 \\
\hline Last & $7.92(4.20)$ & $5.97(3.79)$ & $-13.25 * * *$ & 3370 & .49 \\
\hline
\end{tabular}

Note. At the initial assessment, the sample size was 1005 for recidivists and 2422 for non-recidivists. At the last assessment, the sample size was 999 for recidivists and 2373 for non-recidivists. The RoC*RoI analyses were based on 1002 recidivists and 2418 non-recidivists.

$* p<.05 ; * * * p<.001$.

Maori offenders. As demonstrated in Table 15, the findings for Maori offenders were markedly similar to the full offender sample. Maori offenders with a reconviction had higher levels of static and dynamic risk and lower levels of protective factors compared to those without a reconviction during the study period. Comparable effect sizes were observed for DRAOR subscale scores and factor scores. Effect sizes increased from the initial to last assessment and these differences appear to derive from a larger 
decrease in Stable and Acute Risk and a larger increase in Protective Factors for

offenders who did not incur a reconviction. Lastly, the findings for criminal

reconvictions were similar to the overall sample in that although the effect sizes were

medium to large in magnitude, they were smaller than those for any reconvictions (see

Table 16).

Table 15

Comparison of Recidivists versus Non-recidivists for Maori Offenders

\begin{tabular}{|c|c|c|c|c|c|}
\hline Variable & $\begin{array}{l}\text { Recidivist } \\
M(S D)\end{array}$ & $\begin{array}{c}\text { Non-recidivist } \\
M(S D)\end{array}$ & $t$ & $d f$ & Cohen's d \\
\hline RoC*RoI & $.64(.17)$ & $.48(.23)$ & $-16.49 * * *$ & 1777 & .79 \\
\hline \multicolumn{6}{|c|}{ DRAOR Subscales } \\
\hline \multicolumn{6}{|l|}{ Original Total } \\
\hline Initial & $7.70(6.50)$ & $4.63(5.76)$ & $-10.54 * * *$ & 1780 & .50 \\
\hline Last & $6.04(7.28)$ & $.96(6.57)$ & $-15.30 * * *$ & 1753 & .73 \\
\hline \multicolumn{6}{|l|}{ Stable } \\
\hline Initial & $7.06(2.69)$ & $5.96(2.56)$ & $-8.83 * * *$ & 1780 & .42 \\
\hline Last & $6.47(2.93)$ & $4.83(2.92)$ & $-11.72 * * *$ & 1753 & .56 \\
\hline \multicolumn{6}{|l|}{ Acute } \\
\hline Initial & $6.32(2.48)$ & $5.18(2.20)$ & $-10.26 * * *$ & 1780 & .49 \\
\hline Last & $5.75(2.71)$ & $3.85(2.22)$ & $-15.96 * * *$ & 1753 & .77 \\
\hline \multicolumn{6}{|l|}{ Protective } \\
\hline Initial & $5.69(2.61)$ & $6.52(2.31)$ & $7.11 * * *$ & 1780 & -.34 \\
\hline Last & $6.18(2.88)$ & $7.73(2.61)$ & $11.76 * * *$ & 1753 & -.56 \\
\hline \multicolumn{6}{|l|}{ DRAOR Factors } \\
\hline \multicolumn{6}{|l|}{ Revised Total } \\
\hline Initial & $4.75(6.10)$ & $2.06(5.33)$ & $-9.91 * * *$ & 1780 & .47 \\
\hline Last & $3.36(6.75)$ & $-1.06(6.01)$ & $-14.44 * * *$ & 1753 & .69 \\
\hline \multicolumn{6}{|l|}{ Stable/Acute } \\
\hline Initial & $9.13(3.83)$ & $7.51(3.40)$ & $-9.45 * * *$ & 1780 & .45 \\
\hline Last & $8.30(4.12)$ & $5.74(3.56)$ & $-13.87 * * *$ & 1753 & .66 \\
\hline
\end{tabular}

Note. At the initial assessment, the sample size was 913 for recidivists and 869 for non-recidivists. At the last assessment, the sample size was 907 for recidivists and 848 for non-recidivists. The RoC*RoI analyses were based on 911 recidivists and 868 non-recidivists.

$* p<.05 ; * * * p<.001$. 
Table 16

Comparison of Criminal Recidivists versus Non-recidivists for Maori Offenders

\begin{tabular}{|c|c|c|c|c|c|}
\hline Variable & $\begin{array}{l}\text { Recidivist } \\
M(S D)\end{array}$ & $\begin{array}{c}\text { Non-recidivist } \\
M(S D)\end{array}$ & $t$ & $d f$ & Cohen's d \\
\hline RoC*RoI & $.66(.16)$ & $.51(.22)$ & $-14.62 * * *$ & 1777 & .78 \\
\hline \multicolumn{6}{|c|}{ DRAOR Subscales } \\
\hline \multicolumn{6}{|c|}{ Original Total } \\
\hline Initial & $7.87(6.67)$ & $5.33(5.97)$ & $-8.15 * * *$ & 1780 & .40 \\
\hline Last & $6.07(7.40)$ & $2.27(7.05)$ & $-10.55 * * *$ & 1753 & .53 \\
\hline \multicolumn{6}{|l|}{ Stable } \\
\hline Initial & $7.06(2.72)$ & $6.25(2.62)$ & $-6.11 * * *$ & 1780 & .30 \\
\hline Last & $6.46(2.95)$ & $5.77(3.00)$ & $-8.00 * * *$ & 1753 & .40 \\
\hline \multicolumn{6}{|l|}{ Acute } \\
\hline Initial & $6.47(2.53)$ & $5.40(2.27)$ & $-9.11 * * *$ & 1780 & .45 \\
\hline Last & $5.80(2.76)$ & $4.32(2.46)$ & $-11.50 * * *$ & 1753 & .57 \\
\hline \multicolumn{6}{|l|}{ Protective } \\
\hline Initial & $5.67(2.66)$ & $6.31(2.38)$ & $-5.22 * * *$ & 1780 & -.25 \\
\hline Last & $6.20(2.93)$ & $7.32(2.74)$ & $7.95 * * *$ & 1753 & -.40 \\
\hline \multicolumn{6}{|l|}{ DRAOR Factors } \\
\hline \multicolumn{6}{|l|}{ Revised Total } \\
\hline Initial & $4.88(6.24)$ & $2.70(5.56)$ & $-7.52 * * *$ & 1780 & .37 \\
\hline Last & $3.37(6.86)$ & $.09(6.45)$ & $-9.90 * * *$ & 1753 & .49 \\
\hline \multicolumn{6}{|l|}{ Stable/Acute } \\
\hline Initial & $9.22(3.89)$ & $7.88(3.53)$ & $-7.36 * * *$ & 1780 & .36 \\
\hline Last & $8.30(4.17)$ & $6.41(3.85)$ & $-9.50 * * *$ & 1753 & .47 \\
\hline
\end{tabular}

Note. At the initial assessment, the sample size was 609 for recidivists and 1173 for non-recidivists. At the last assessment, the sample size was 607 for recidivists and 1148 for non-recidivists. The RoC*RoI analyses were based on 608 recidivists and 1171 non-recidivists.

$* * * p<.001$.

Summary: Part 3. In support of Hypothesis 2, reconvicted offenders had higher Stable Risk, Acute Risk, and DRAOR Total scores, and lower Protective Factors. These findings were consistent across a number of different comparisons by assessment sequence (initial, last), sample (overall, Maori), and criteria to categorize groups (all reconvictions, criminal reconvictions). 


\section{Part 4. Prediction of Recidivism from Dynamic and Static Measures Predictive accuracy of the DRAOR.}

Hypothesis 3a. Recidivism can be predicted from stable and acute dynamic risk factors.

Hypothesis 3b. Desistance from crime (i.e., no reconvictions during follow-up) will be best predicted from protective factors.

The first part of the empirical litmus test to demonstrate that the DRAOR is in fact dynamic involves establishing the predictive accuracy of the subscale scores in relation to recidivism. ROC analysis was used to examine the predictive accuracy of the DRAOR and its subscales in regards to reconvictions and criminal reconvictions in particular. These analyses focused on the last DRAOR assessment for each offender prior to reconviction date. This assessment was selected as it was expected to demonstrate the highest predictive accuracy, being most proximal to outcome. This decision was reinforced by the previous between-offender findings in which the last DRAOR assessment differentiated recidivists and non-recidivists better than their first assessment. The first reconviction occurring after the DRAOR assessment was used as the outcome variable. Rice and Harris (2005) suggest that AUCs of .55 to .63 are indicative of small effect sizes, .64 to .70 are medium, and .71 and higher are large effect sizes. Meanwhile, Gendreau and Goggin (1996) advise that good risk measures can predict recidivism in the $r=.30-.45$ range. $^{12}$

During the follow up period, $42.6 \%$ of the sample had a reconviction and $28.9 \%$ had a criminal reconviction after being assessed on the DRAOR. Base rates of

\footnotetext{
${ }^{12}$ Other commonly used guidelines do not consider an AUC of .71 to be high (e.g., Swets, 1986; Hosmer \& Lemeshow, 2000). However, the guidelines of Rice and Harris (2005) and Gendreau and Goggin (1996) were selected as they are specific to the field of Forensic Psychology.
} 
recidivism were higher for Maori offenders than for the overall sample, with $50.2 \%$ of offenders being reconvicted and $33.8 \%$ being reconvicted for a criminal offence. The mean time from first assessment to first reconviction was 143.78 days $(S D=135.35)$ days for the overall sample and $136.93(S D=128.47)$ days for Maori offenders. The mean time to first criminal reconviction was $173.96(S D=149.37)$ days for the overall sample and $168.48(S D=143.63)$ days for Maori offenders. Appendix G displays the base rates for all reconvictions and criminal reconvictions by DRAOR total score.

The predictive accuracy results are presented in Table 17 for the overall sample and in Table 18 for Maori offenders. The DRAOR and its subscales consistently predicted the reconviction outcomes to varying degrees, with small to medium effect sizes. Larger effect sizes were observed for reconvictions than criminal reconvictions, with AUC values ranging from .66 to .72 for reconvictions and .62 to .67 for criminal reconvictions in the overall sample. The effect sizes for the Maori offender subgroup were slightly lower, with AUC values ranging from .65 to .70 for reconvictions and .61 to .66 for criminal reconvictions. The DRAOR total score and the Acute Risk subscale in particular demonstrated the best predictive accuracy for both the overall sample and Maori offenders in particular. The predictive accuracy of the original DRAOR total score was slightly higher than the revised DRAOR total score for each of the samples and outcome measures. Likewise, the Acute Risk subscale was markedly more accurate than the mixed Stable/Acute factor. 
Table 17

Predictive Accuracy of the DRAOR: Overall Sample $(N=3372)$

\begin{tabular}{|c|c|c|c|c|c|}
\hline \multicolumn{6}{|l|}{ Reconviction Type } \\
\hline DRAOR Measure & Base rate & $r$ & AUC & SE & $95 \% \mathrm{CI}$ \\
\hline Any Reconviction $(n=1436)$ & $42.6 \%$ & & & & \\
\hline DRAOR Total & & .36 & .71 & .01 & {$[.69, .73]$} \\
\hline Stable & & .29 & .66 & .01 & {$[.65, .68]$} \\
\hline Acute & & .38 & .72 & .01 & {$[.70, .74]$} \\
\hline Protective & & .29 & .67 & .01 & {$[.65, .68]$} \\
\hline Revised Total & & .35 & .70 & .01 & {$[.68, .72]$} \\
\hline Stable/Acute & & .32 & .69 & .01 & {$[.67, .70]$} \\
\hline Criminal Reconviction $(n=976)$ & $28.9 \%$ & & & & \\
\hline DRAOR Total & & .27 & .66 & .01 & {$[.64, .68]$} \\
\hline Stable & & .21 & .62 & .01 & {$[.60, .64]$} \\
\hline Acute & & .29 & .67 & .01 & {$[.65, .69]$} \\
\hline Protective & & .21 & .62 & .01 & {$[.60, .64]$} \\
\hline Revised Total & & .26 & .65 & .01 & {$[.63, .67]$} \\
\hline Stable/Acute & & .24 & .64 & .01 & {$[.62, .66]$} \\
\hline
\end{tabular}

Table 18

Predictive Accuracy of the DRAOR: Maori Offenders $(N=1755)$

\begin{tabular}{lccccc}
\hline $\begin{array}{l}\text { Reconviction Type } \\
\text { DRAOR Measure }\end{array}$ & Base rate & $r$ & AUC & SE & $95 \%$ CI \\
\hline Any Reconviction $(n=881)$ & $50.2 \%$ & & & & \\
$\quad$ DRAOR Total & & .33 & .69 & .01 & {$[.67, .72]$} \\
Stable & & .26 & .65 & .01 & {$[.62, .67]$} \\
Acute & & .35 & .70 & .01 & {$[.68, .72]$} \\
Protective & .26 & .65 & .01 & {$[.62, .67]$} \\
Revised Total & & .32 & .68 & .01 & {$[.66, .71]$} \\
Stable/Acute & & .30 & .67 & .01 & {$[.65, .70]$} \\
& & & & & \\
Criminal Reconviction $(n=594)$ & $33.8 \%$ & & & & \\
DRAOR Total & & .26 & .65 & .01 & {$[.62, .68]$} \\
Stable & & .21 & .61 & .01 & {$[.59, .64]$} \\
Acute & & .27 & .66 & .01 & {$[.63, .68]$} \\
Protective & & .20 & .61 & .01 & {$[.58, .64]$} \\
Revised Total & & .25 & .64 & .01 & {$[.61, .67]$} \\
Stable/Acute & & .23 & .63 & .01 & {$[.61, .66]$} \\
\hline
\end{tabular}

Note. All predictive accuracy statistics significant at $p<.001 ; \mathrm{AUC}=$ area under the curve; $\mathrm{SE}=$ standard error; $\mathrm{CI}=$ confidence interval. 
Relationship with static risk. A between-subjects ANCOVA was first performed to test whether the number of DRAOR assessments differed by RoC*RoI static risk group, controlling for time in the community. After adjusting for the number of days between parole start and follow-up end (either recidivism or censoring), there were no significant differences between static risk groups in the number of assessments, $F(3,3483)=1.83, p=.14$. This analysis was repeated by grouping the number of assessments into three-month increments following parole start date. From parole start to three months following, there was a significant difference between static risk groups in the number of assessments, $F(3,3488)=7.138, p<.001$. Pairwise comparisons revealed that low risk offenders $(M=11.03, S D=4.57)$ had fewer assessments than medium $(M=$ $11.44, S D=4.80)$ and high $(M=11.41, S D=5.55)$ risk offenders, consistent with parole supervision guidelines. Significant differences were also observed in the three to six months following parole $\operatorname{start}(F(3,3488)=3.809, p=.01)$, again with low risk $(M=$ $6.48, S D=4.11)$ having fewer assessments than medium $(M=6.32, S D=4.68)$ and high $(M=6.09, S D=4.97)$ risk offenders. Following six months, there were no differences between static risk groups.

Hypothesis 4. The DRAOR will demonstrate good convergent validity with the $R o C^{*} R o I$.

Convergent validity was assessed using correlational data between the DRAOR and $\mathrm{RoC}^{*} \mathrm{RoI}$ as a measure of static risk. All of the correlations reported are significant at the $p<.001$ level. As expected, the DRAOR total score and the RoC*RoI were significantly correlated at the initial $(r=.32)$ and last $(r=.37)$ DRAOR assessments prior to recidivism or the study end date. For all of the DRAOR subscales, the correlations 
with static risk increased from the initial to the last assessment. Stable Risk demonstrated the largest correlation with Static Risk (from $r=.31$ at initial to $r=.35$ at last assessment), followed by Protective Factors (from $r=-.25$ at initial to $r=-.31$ at last assessment), and Acute Risk (from .24 at initial to .30 at last assessment). The DRAOR Revised Total score showed smaller correlations with the Roc*RoI than the original Total score at the initial $(r=.28)$ and last $(r=.33)$ assessments. The combined Stable/Acute factor had a correlation of .32 at initial and .33 at last assessment.

Hypothesis 5. The DRAOR will predict recidivism above and beyond the RoC*RoI, but the combined dynamic and static model will demonstrate the greatest accuracy.

Cox regression was used to assess the incremental validity of the DRAOR Total score compared to the RoC*RoI. Table 19 presents the results for all reconvictions and criminal reconvictions separately for the overall sample and Maori offenders. Only the results for the original DRAOR total score are presented as the pattern of findings based on the revised DRAOR total score were similar. For the prediction of both all reconvictions and criminal reconvictions, the DRAOR was found to add incrementally to the RoC*RoI. The incremental contributions were consistently positive. For instance, the odds ratio of the DRAOR for the model predicting all reconvictions in the overall sample was 1.04. This indicates that each one-score increase on the DRAOR increases the hazard (i.e., the occurrence of reconvictions) by $4 \%$, after controlling for the RoC*RoI. 
Table 19

Incremental Validity of the DRAOR and RoC*RoI for Predicting All Reconvictions and Criminal Reconvictions

\begin{tabular}{|c|c|c|c|c|}
\hline $\begin{array}{l}\text { Reconviction Type } \\
\text { Sample }\end{array}$ & $N$ & Comparison & $\mathrm{B}$ & $\begin{array}{c}\text { Hazard } \\
\text { Ratio }\end{array}$ \\
\hline \multicolumn{5}{|l|}{ All Reconvictions } \\
\hline \multirow[t]{2}{*}{ Overall sample } & 3355 & DRAOR & $.04 * * *$ & 1.04 \\
\hline & & RoC*RoI & $1.58 * * *$ & 4.86 \\
\hline \multirow[t]{2}{*}{ Maori offenders } & 1746 & DRAOR & $.03 * * *$ & 1.03 \\
\hline & & RoC*RoI & $1.58 * * *$ & 4.84 \\
\hline \multicolumn{5}{|c|}{ Criminal Reconvictions } \\
\hline \multirow[t]{2}{*}{ Overall sample } & 3339 & DRAOR & $.03 * * *$ & 1.03 \\
\hline & & RoC*RoI & $2.33 * * *$ & 10.27 \\
\hline \multirow[t]{2}{*}{ Maori offenders } & 1707 & DRAOR & $.03 * * *$ & 1.03 \\
\hline & & RoC*RoI & $2.29 * * *$ & 9.86 \\
\hline
\end{tabular}

Note. Analyses conducted separately for each comparison, with both risk instruments entered simultaneously in the model. Sample size fluctuates because of the amount of cases censored before earliest event.

$* * * p<.001$.

The predictive accuracy of the RoC*RoI was compared to two combined dynamic and static models, which were expected to demonstrate the highest predictive accuracy. The first combined dynamic and static model was calculated by equally weighting the RoC*RoI and the DRAOR Total Score. A second combined dynamic and static model was calculated by equally weighting the RoC*RoI and the DRAOR Revised Total Score. $^{13}$

As demonstrated in Table 20, the AUCs were higher for static risk than those of dynamic risk both in terms of all reconvictions and criminal reconvictions. Table 21

\footnotetext{
${ }^{13}$ The scales were also combined by entering them in a logistic regression analysis, producing predicted recidivism estimates and performing ROC analyses on the predicted values. Although this is a more precise method of combining scales to enhance predictive validity, the resulting AUCs were the same as the more simplistic method of equally weighting the scales. The results for this more simplistic and practically appealing method are presented.
} 
presents the test of differences in AUCs between scales using the Delong method. The Delong test found that the combined dynamic and static models had significantly greater discrimination than the $\mathrm{RoC} * \mathrm{RoI}$ in predicting all reconvictions. However, there was no significant difference in the predictive accuracy of the $\mathrm{RoC}^{*} \mathrm{RoI}$ compared to the combined dynamic and static models when predicting criminal convictions. For both all reconvictions and criminal reconvictions, the combined dynamic and static model using the original DRAOR total score was superior in predictive accuracy to that using the revised DRAOR total score.

Table 20

Predictive Accuracy of the RoC*RoI $(N=3372)$ and Combined Static and Dynamic Models $(N=3365):$ Overall Sample

\begin{tabular}{|c|c|c|c|c|c|c|}
\hline \multirow{2}{*}{$\begin{array}{l}\text { Reconviction Type } \\
\text { Model }\end{array}$} & \multicolumn{2}{|c|}{ Base rate } & \multirow[b]{2}{*}{$r$} & \multirow[b]{2}{*}{ AUC } & \multirow[b]{2}{*}{ SE } & \multirow[b]{2}{*}{$95 \% \mathrm{CI}$} \\
\hline & $n$ & $\%$ & & & & \\
\hline \multicolumn{7}{|l|}{ All Reconvictions } \\
\hline RoC*RoI & 1436 & 42.6 & .40 & .73 & .01 & {$[.71, .75]$} \\
\hline Original dynamic static model & 1432 & 42.6 & .46 & .77 & .01 & {$[.75, .78]$} \\
\hline Revised dynamic static model & 1432 & 42.6 & .45 & .76 & .01 & {$[.74, .78]$} \\
\hline \multicolumn{7}{|l|}{ Criminal Reconvictions } \\
\hline RoC*RoI & 976 & 28.9 & .40 & .73 & .01 & {$[.71, .75]$} \\
\hline Original dynamic static model & 976 & 29.0 & .41 & .74 & .01 & {$[.72, .75]$} \\
\hline Revised dynamic static model & 976 & 29.0 & .40 & .73 & .01 & {$[.71, .75]$} \\
\hline
\end{tabular}

Note. All predictive accuracy statistics significant at $p<.001 ; \mathrm{AUC}=$ area under the curve; $\mathrm{SE}=$ standard error; $\mathrm{CI}=$ confidence interval. 
Table 21

Comparison of Static and Dynamic Models: Overall Sample $(N=3365)$

\begin{tabular}{|c|c|c|c|}
\hline $\begin{array}{l}\text { Reconviction Type } \\
\text { Scale Comparison }\end{array}$ & AUC & $95 \% \mathrm{CI}$ & $\chi^{2}$ \\
\hline \multicolumn{4}{|l|}{ All Reconvictions } \\
\hline Original dynamic static model vs. RoC*RoI & .0390 & {$[.0269, .0511]$} & $39.96 * * *$ \\
\hline Revised dynamic static model vs. RoC*RoI & .0315 & {$[.0190, .0441]$} & $24.23 * * *$ \\
\hline Original vs. revised dynamic static model & .0075 & {$[.0057, .0093]$} & $68.02 * * *$ \\
\hline \multicolumn{4}{|l|}{ Criminal Reconvictions } \\
\hline Original dynamic static model vs. RoC*RoI & .0033 & {$[-.0103, .0168]$} & .22 \\
\hline Revised dynamic static model vs. RoC*RoI & -.0044 & {$[-.0185, .0096]$} & .38 \\
\hline Original vs. revised dynamic static model & .0077 & {$[.0058, .0096]$} & $60.05 * * *$ \\
\hline
\end{tabular}

Note. $\mathrm{AUC}=$ area under the curve; $\mathrm{CI}=$ confidence interval. A positive AUC difference score indicates greater discrimination by the first scale compared to the second scale in the comparison. $p<.001$.

The same pattern of results was found for Maori offenders. The RoC*RoI showed higher AUCs than the DRAOR for Maori offenders for both reconvictions and criminal reconvictions (see Table 22). The Delong tests presented in Table 23 indicate that the combined dynamic and static models predicted all reconvictions more accurately than the RoC*RoI, but not criminal reconvictions. Lastly, the combined dynamic and static model using the original DRAOR total score predicted both reconvictions and criminal reconvictions with greater discrimination than the model using the revised DRAOR total score. 
Table 22

Predictive Accuracy of the RoC ${ }^{*} \operatorname{RoI}(N=1755)$ and Combined Static and Dynamic Models $(N=1751)$ : Maori Offenders

\begin{tabular}{|c|c|c|c|c|c|c|}
\hline \multirow{2}{*}{$\begin{array}{l}\text { Reconviction Type } \\
\text { Model }\end{array}$} & \multicolumn{2}{|c|}{ Base rate } & \multirow[b]{2}{*}{$r$} & \multirow[b]{2}{*}{ AUC } & \multirow[b]{2}{*}{ SE } & \multirow[b]{2}{*}{$95 \% \mathrm{CI}$} \\
\hline & $n$ & $\%$ & & & & \\
\hline \multicolumn{7}{|l|}{ Any Reconvictions } \\
\hline RoC*RoI & 881 & 50.2 & .35 & .70 & .01 & {$[.67, .72]$} \\
\hline Original dynamic static model & 878 & 50.1 & .41 & .74 & .01 & {$[.71, .76]$} \\
\hline Revised dynamic static model & 878 & 50.1 & .40 & .73 & .01 & {$[.71, .75]$} \\
\hline \multicolumn{7}{|l|}{ Criminal Reconvictions } \\
\hline RoC*RoI & 594 & 33.8 & .35 & .70 & .01 & {$[.67, .72]$} \\
\hline Original dynamic static model & 594 & 34.0 & .36 & .71 & .01 & {$[.69, .73]$} \\
\hline Revised dynamic static model & 594 & 34.0 & .35 & .70 & .01 & {$[.68, .73]$} \\
\hline
\end{tabular}

Note. All predictive accuracy statistics significant at $p<.001 ; \mathrm{AUC}=$ area under the curve; $\mathrm{SE}=$ standard error; $\mathrm{CI}=$ confidence interval.

Table 23

Comparison of Static and Dynamic Models: Maori Offenders $(N=1751)$

\begin{tabular}{|c|c|c|c|}
\hline $\begin{array}{l}\text { Reconviction Type } \\
\text { Scale Comparison }\end{array}$ & AUC & $95 \% \mathrm{CI}$ & $\chi^{2}$ \\
\hline \multicolumn{4}{|l|}{ All Reconvictions } \\
\hline Original dynamic static model vs. RoC*RoI & .0412 & {$[.0228, .0596]$} & $19.26 * * *$ \\
\hline Revised dynamic static model vs. RoC*RoI & .0336 & {$[.0146, .0526]$} & $12.01 * * *$ \\
\hline Original vs. revised dynamic static model & .0076 & {$[.0049, .0102]$} & $31.77 * * *$ \\
\hline \multicolumn{4}{|l|}{ Criminal Reconvictions } \\
\hline Original dynamic static model vs. RoC*RoI & .0013 & {$[-.0070, .0329]$} & 1.62 \\
\hline Revised dynamic static model vs. RoC*RoI & .0052 & {$[-.0154, .0259]$} & .25 \\
\hline Original vs. revised dynamic static model & .0077 & {$[.0050, .0105]$} & $30.56 * * *$ \\
\hline
\end{tabular}

Note. AUC $=$ area under the curve; $\mathrm{CI}=$ confidence interval. A positive AUC difference score indicates greater discrimination by the first scale compared to the second scale in the comparison. $p<.001$.

Summary: Part 4. The DRAOR subscales and total score consistently predicted reconvictions and criminal reconvictions, with the Acute Risk subscale demonstrating the highest predictive accuracy. This provides partial support for Hypothesis 3 in that 
recidivism was predicted from stable and acute dynamic risk factors, but protective factors were not the best predictors of desistance from crime. Consistent with Hypothesis 4, the DRAOR demonstrated good convergent validity with the RoC*RoI. Lastly, the DRAOR predicted reconvictions and criminal reconvictions above and beyond the RoC*RoI, but the combined dynamic and static model demonstrated the greatest accuracy for reconvictions only. This provides partial support for Hypothesis 5.

The findings suggest that in terms of psychometric properties and predictive accuracy, the original DRAOR and its subscales appear to be superior to the revised subscales developed through factor analysis. In light of this, the remaining analyses will be performed on the original DRAOR subscales only.

\section{Part 5. Change in Dynamic Risk Over a Period of Assessment and Reassessment}

Hypothesis 6. Dynamic factors will change over a period of systematic assessment and reassessment within an individual offender.

Model building process. ROC analyses showed that DRAOR scores are significantly related to recidivism. The second part of the empirical litmus test requires evidence that the DRAOR subscale scores change over time. Multilevel growth modelling was used to examine the dynamic nature of the DRAOR over time to determine whether scores changed over time. Time was used as a level-1 covariate, allowing for an examination of change across time. This analysis provides an integrated approach for studying the structure and indicators of individual growth. Growth models provide an understanding of average growth trends across time in the outcome variables (e.g., offenders' average score at the starting point and how much they change), as well as an understanding of person variables that explain variability in growth (e.g., static risk). 
In order to be included in the analysis, offenders were required to have a minimum of two assessments following their baseline third assessment. Any assessments following first reconviction were excluded from the analysis in the interest of conceptual clarity. The mean number of DRAOR assessments per offender was $29.06(S D=16.13)$, with a range from 5 to 120 . The number of days between the first assessment and last assessment ranged from 8 to 782 , with recidivists assessed over a longer period than nonrecidivists $(M=325.63, S D=186.69$ and $M=279.81, S D=177.78$, respectively).

Missing data was deleted when creating the models. This resulted in 138 assessments deleted. This allowed for full maximum likelihood estimation to be used and important goodness of fit statistics (i.e., deviance statistic, AIC and Bayesian Information Criterion (BIC)) to be calculated and compared. Full maximum likelihood (FML) estimation assesses the probability of simultaneously observing the entire sample data actually obtained (Singer \& Willett, 2003). The estimates of all the population parameters are jointly maximized using FML estimation. The disadvantage of this method of estimation is that it overstates the degrees of freedom left for the variance components and thus underestimates the variance components, but this is more of an issue in small samples.

Three growth models were created using each of the original DRAOR subscales (Stable Risk, Acute Risk, and Protective Factors) as outcome variables. First, an unconditional means model (i.e., null model with no time variables or predictors entered) was created for each of the outcome variables. These models establish whether there is systematic variation in Stable Risk, Acute Risk, and Protective Factors worth investigating further, and provides an important baseline to which subsequent models are 
compared (Singer \& Willett, 2003). Intra-class correlations will be presented as a measure of the proportion of total variation in outcome that occurs between offenders.

Next, an unconditional growth model was developed for each of the outcome variables by adding a time predictor. These models partition and quantify outcome variation across people and time to determine the extent to which it resides within and between offenders (Singer \& Willett, 2003). These models answer the level-1 question regarding how dynamic factors change over time. The time variable used was the number of days since the first assessment, centered at the first assessment (i.e., coded zero). This was to allow for an examination of the initial status in dynamic risk and protective factors, as well as the rate of change over the course of assessments. Thus, intercepts represent the DRAOR subscale scores at first assessment and the slopes represent the relationship between DRAOR subscale scores and time. Although other forms of time were explored (e.g., squared, cubed), a linear model best fit the data for each of the growth models. In addition, there was variability around time in that there were differences between offenders for the intercepts (inclusion of $\sigma^{2}{ }_{0}$ in the models) and slopes (inclusion of $\sigma^{2}{ }_{1}$ in the models).

Lastly, level-2 variables were explored to determine whether offenders' dynamic factors could be explained by between-offender variables. A number of level-2 betweenoffender variables were explored including the RoC*RoI, Maori ethnicity, gender, and age. In addition, potential control variables included the number of days between the first and last assessment, the number of days between the first assessment and follow-up date, as well as the total number of assessments. Dichotomous variables (i.e., Maori ethnicity and gender) were entered into the model uncentered such that the level-2 fitted intercepts 
represent the value of level-1 individual growth parameters when all predictors in the associated level-2 model are zero. The remaining variables were grand mean centered. As recommended by Singer and Willett (2003), decisions to enter, retain, and remove predictors were based on logic, theory, and prior research, supplemented by rigorous hypothesis testing and comparisons of model fit. For each of the outcome variables, a taxonomy of statistical models will be presented. As a set, this systematic sequence of models will demonstrate the model building process with the best predictors of the outcome variables. For each of the models, other level-2 variables were found to better account for variance in the dynamic risk and protective factor outcomes than Maori ethnicity. As such, the analyses were not repeated for Maori offenders after building models for all offenders.

To assess goodness of fit and compare models, deviance statistics, AIC, and BIC were used. The deviance statistic compares the log-likelihood statistics for the current model fit and a saturated model that fits the data perfectly (Singer \& Willett, 2003). Deviance statistics can only be used to compare models that are nested within each other. On the other hand, AIC and BIC are used to compare the goodness of fit for non-nested models (i.e., with different sets of predictors).

Pseudo $\mathrm{R}^{2}$ statistics are presented which quantify how much outcome variation is explained by each of the growth model's predictors (Singer \& Willett, 2003). Pseudo $\mathrm{R}_{\mathrm{y}, \mathrm{y}}^{2}$ provides the proportion of total outcome variation in Stable Risk, Acute Risk, and Protective Factors explained by their multilevel models' combination of predictors. Pseudo $\mathrm{R}^{2} \varepsilon$ assesses the proportion of within-offender variation explained by time. Given that there are no level-1 time-varying predictors, this statistic remains constant across 
models for each of the outcomes. Pseudo $\mathrm{R}_{0}^{2}$ and $\mathrm{R}^{2}{ }_{1}$ quantify the proportional reduction in level-2 residual variance with the addition of predictors at the intercepts and slopes.

Lastly, the tenability of the model's assumptions was evaluated for each of the models presented. Most of the assumptions had previously been tested at level-1 in the Statistical and Methodological Issues section. The assumptions (e.g., normality, linearity, homoscedasticity) were also evaluated at level-2 with no violations present.

Stable risk. The unconditional means model (Model A), unconditional growth model (Model B), and potential level-2 between-offender models (Models C and D) are presented in Table 24 and their results presented in Table 25. In the unconditional means model, the grand mean of Stable Risk across all assessment occasions and offenders is 5.46. The variance components are significantly different from zero, therefore the average parolee's Stable Risk varies over time $\left(\sigma^{2}{ }_{\varepsilon}=2.07, p<.001\right)$ and parolees differ from each other in Stable Risk $\left(\sigma^{2}{ }_{0}=6.86, p<.001\right)$. Therefore, there is variation at both level-1 and level-2 that can potentially be explained. The intra-class correlation is .77, indicating that $77 \%$ of variance in Stable Risk occurs between offenders at level-2.

The unconditional growth model (Model B) demonstrates how offenders change over time. The average Stable Risk score across all offenders at first assessment was 5.72. The average slope across the population was -.003 , indicating that on average, the Stable Risk score decreased by .003 per day after first assessment. The level-1 residual variance shows a scatter of 1.21 of each offender's data around their own linear change trajectory. This residual variance is smaller than that of the unconditional means model, which indicates that the unconditional growth model better predicts observed Stable Risk scores. A decline of .86 in level-1 residual variance from Model A to Model B indicates 
that $86 \%$ of within-offender variation in Stable Risk is systematically associated with linear time. The level-2 residual variances summarize between-offender variability of 7.23 in initial status at first assessment and .0001 in rate of change across the course of assessments. Both of these variance components are significant, indicating that interindividual differences in change are due to differences in both true initial status and true rate of change. Pseudo $\mathrm{R}^{2} \varepsilon$ indicates the proportion of within-offender variation explained by time is $42 \%$.

Two level-2 models are presented which explore several variables that might explain different initial values at first assessment. There were no level-2 variables that were found to significantly explain different rates of change in Stable Risk between offenders. Model C includes RoC*RoI as a predictor of initial status only. The fixed effects for initial status and rate of change intercepts do not change drastically with the addition of this between-offender predictor. As static risk increases, the change in the estimated mean of Stable Risk score of the entire parolee population increases by 1.37. Model D evaluates the effects of RoC*RoI on initial status of Stable Risk, controlling for the effects of Age. Once removing the effects of Age, the intercept for initial status does not change but the fixed effect for RoC*RoI increases by $44 \%$. The fixed effect for Age describes a differential of .03 in Stable Risk score for a one-unit difference in Age, controlling for the effect of Static Risk. Both Models C and D indicate that parolees with higher static risk at release initially have higher Stable Risk scores at first assessment. The magnitude of the early differential in Stable Risk is higher after Age is controlled. 
Figure 4 displays a set of prototypical change trajectories for an offender using each of the models. ${ }^{14}$ Deviance-based hypothesis tests indicate an improvement from Model B to Model C $\left(\chi^{2}(4)=35852.4, p<.001\right)$ and from Model C to Model D $\left(\chi^{2}(1)=\right.$ $30.75, p<.001)$. AIC and BIC statistics show that Model D demonstrates the best fit. The decline in residual variation from Model B to Models C and D is small, suggesting that although there is an improvement in fit, the additional predictors do not make a large contribution. The statistically significant level-1 within-person variance components for Models C and D are identical to Model B, reinforcing the need to explore the effects of within-offender time-varying predictors. The level-2 variance in initial status only decreased by $1.5 \%$ from Model B to Model C and .8\% from Model C to Model D. Both of these variance components are still statistically significant, meaning that there is remaining potentially explainable residual variation in initial status. This is also true for the level-2 variance in rate of change as no predictors were added to the slopes. Finally, the Pseudo $\mathrm{R}_{\mathrm{y}, \mathrm{y}}^{2}$ statistics indicate that the amount of variation in Stable Risk accounted for by the growth models' predictors' is only $1 \%$ and does not change from Models $\mathrm{C}$ to $\mathrm{D}$ at $8 \%$. Model $\mathrm{D}$ is thus selected as a tentative final model, with the caveat that further model building is required, particularly focused on level-2 predictors to account for the $77 \%$ of variance occurring between offenders.

\footnotetext{
${ }^{14}$ Prototypical changes trajectories were developed based on the mean number of assessments $(M=37.32)$ and the mean number of days since the first assessments $(M=161.73)$. These were divided to obtain a mean number of days between assessments $(M=4.33)$. Stable risk scores were then calculated for 37 assessments for an offender with 4.33 days between each assessment. The mean $\operatorname{RoC} * \operatorname{RoI}$ score $(M=.50)$ and mean Age $(M=35.52)$ were imputed into Models C and D.
} 
Table 24

Taxonomy of Multilevel Models for Change Fitted to Stable Risk Data

Level-1/Level-2 Specification

\begin{tabular}{|c|c|c|c|}
\hline Model & Level-1 Model & Level-2 Model & Composite Model \\
\hline A & STABLE $_{\mathrm{ti}}=\pi_{0 \mathrm{i}}+\mathrm{e}_{\mathrm{ti}}$ & $\pi_{0 \mathrm{i}}=\beta_{00}+\mathrm{r}_{0 \mathrm{i}}$ & STABLE $_{t i}=\beta_{00}+r_{0 i}+e_{t i}$ \\
\hline B & $\operatorname{STABLE}_{\mathrm{ti}}=\pi_{0 \mathrm{i}}+\pi_{1 \mathrm{i}} *\left(\mathrm{DAYS}_{\mathrm{ti}}\right)+\mathrm{e}_{\mathrm{ti}}$ & $\begin{array}{l}\pi_{0 \mathrm{i}}=\beta_{00}+\mathrm{r}_{0 \mathrm{i}} \\
\pi_{1 \mathrm{i}}=\beta_{10}+\mathrm{r}_{1 \mathrm{i}}\end{array}$ & $\operatorname{STABLE}_{\mathrm{ti}}=\beta_{00}+\beta_{10} * \mathrm{DAYS}_{\mathrm{ti}}+\mathrm{r}_{0 \mathrm{i}}+\mathrm{r}_{1 \mathrm{i}} * \mathrm{DAYS}_{\mathrm{ti}}+\mathrm{e}_{\mathrm{ti}}$ \\
\hline $\mathrm{C}$ & $\mathrm{STABLE}_{\mathrm{ti}}=\pi_{0 \mathrm{i}}+\pi_{1 \mathrm{i}} *\left(\mathrm{DAYS}_{\mathrm{ti}}\right)+\mathrm{e}_{\mathrm{ti}}$ & $\begin{array}{c}\pi_{0 \mathrm{i}}=\beta_{00}+\beta_{01} *\left(\mathrm{ROC}_{\mathrm{i}}\right)+\mathrm{r}_{0 \mathrm{i}} \\
\pi_{1 \mathrm{i}}=\beta_{10}+\mathrm{r}_{1 \mathrm{i}}\end{array}$ & $\operatorname{STABLE}_{\mathrm{ti}}=\beta_{00}+\beta_{01} * \mathrm{ROC}_{\mathrm{i}}+\beta_{10} * \mathrm{DAYS}_{\mathrm{ti}}+\mathrm{r}_{0 \mathrm{i}}+\mathrm{r}_{1 \mathrm{i}} * \mathrm{DAYS}_{\mathrm{ti}}+\mathrm{e}_{\mathrm{t}}$ \\
\hline $\mathrm{D}$ & $\operatorname{STABLE}_{\mathrm{ti}}=\pi_{0 \mathrm{i}}+\pi_{1 \mathrm{i}} *\left(\mathrm{DAYS}_{\mathrm{ti}}\right)+\mathrm{e}_{\mathrm{ti}}$ & $\begin{array}{c}\pi_{0 \mathrm{i}}=\beta_{00}+\beta_{01} *\left(\mathrm{ROC}_{\mathrm{i}}\right)+\beta_{02} *\left(\mathrm{AGE}_{\mathrm{i}}\right)+\mathrm{r}_{0 \mathrm{i}} \\
\pi_{1 \mathrm{i}}=\beta_{10}+\mathrm{r}_{1 \mathrm{i}}\end{array}$ & $\begin{array}{l}\mathrm{STABLE}_{\mathrm{ti}}=\beta_{00}+\beta_{01} * \mathrm{ROC}_{\mathrm{i}}+\beta_{02} * \mathrm{AGE}_{\mathrm{i}}+\beta_{10} * \mathrm{DAYS}_{\mathrm{ti}}+\mathrm{r}_{0 \mathrm{i}}+ \\
\mathrm{r}_{1 \mathrm{i}} * \mathrm{DAYS}_{\mathrm{ti}}+\mathrm{e}_{\mathrm{ti}}\end{array}$ \\
\hline
\end{tabular}

Note. These models predict Stable Risk in all New Zealand parolees as a function of days since first assessment (at level-1) and various combinations of RoC*RoI and Age (at level-2). Models C and D enter the level-2 predictors in grand mean centred form. 
Table 25

Results of Fitting a Taxonomy of Multilevel Models for Change for Stable Risk $(N=$ 3317)

\begin{tabular}{|c|c|c|c|c|c|c|}
\hline \multirow{2}{*}{\multicolumn{2}{|c|}{ Fixed Effects }} & Parameter & Model A & Model B & Model C & Model D \\
\hline & & & \multicolumn{4}{|c|}{ Estimate (Standard Error) } \\
\hline \multirow{6}{*}{$\begin{array}{l}\text { Initial } \\
\text { status, } \\
\pi_{0 \mathrm{i}}\end{array}$} & Intercept & $\gamma_{00}$ & $5.46 * * *$ & $5.72 * * *$ & $5.72 * * *$ & $5.72 * * *$ \\
\hline & & & $(.05)$ & $(.05)$ & $(.05)$ & $(.05)$ \\
\hline & Roc*RoI & $\gamma_{01}$ & & & $1.37 * * *$ & $1.97 * * *$ \\
\hline & & & & & $(.19)$ & $(.21)$ \\
\hline & Age & $\gamma_{02}$ & & & & $.03 * * *$ \\
\hline & & & & & & $(.004)$ \\
\hline \multirow{3}{*}{$\begin{array}{l}\text { Rate of } \\
\text { change, } \\
\pi_{1 \mathrm{i}}\end{array}$} & Intercept & $\gamma_{10}$ & & $-.003 * * *$ & $-.003 * * *$ & $-.003 * * *$ \\
\hline & & & & $(.0002)$ & $(.0002)$ & $(.0002)$ \\
\hline & & & & & & \\
\hline \multicolumn{3}{|c|}{ Variance Components } & \multicolumn{4}{|c|}{ Estimate (Standard Error) } \\
\hline \multirow[t]{2}{*}{ Level 1} & Within- & $\sigma^{2} \varepsilon$ & $2.07 * * *$ & $1.21 * * *$ & $1.21 * * *$ & $1.21 * * *$ \\
\hline & person & & $(1.44)$ & $(1.10)$ & $(1.10)$ & $(1.10)$ \\
\hline \multirow[t]{4}{*}{ Level 2} & In initial & $\sigma_{0}^{2}$ & $6.86^{* * *}$ & $7.23 * * *$ & $7.12 * * *$ & $7.07 * * *$ \\
\hline & status & & $(2.62)$ & $(2.69)$ & $(2.67)$ & $(2.66)$ \\
\hline & In rate of & $\sigma_{1}^{2}$ & & $.0001 * * *$ & $.0001 * * *$ & $0001 * * *$ \\
\hline & change & & & $(.01)$ & $(.01)$ & $(.01)$ \\
\hline \multicolumn{7}{|c|}{ Pseudo $\mathrm{R}^{2}$ Statistics and Goodness-of-fit } \\
\hline & $\mathrm{R}_{\mathrm{y}, \mathrm{y}}^{2}$ & & - & .01 & .08 & .08 \\
\hline & $\mathrm{R}_{\varepsilon}^{2}$ & & - & .42 & .42 & .42 \\
\hline & $\mathrm{R}_{0}^{2}$ & & - & - & .02 & .02 \\
\hline & Deviance & & 318449.9 & 282651.8 & 282597.7 & 282566.9 \\
\hline & $\mathrm{AIC}$ & & 318455.9 & 282663.8 & 282597.7 & 282582.9 \\
\hline & $\mathrm{BIC}$ & & 318483.9 & 282719.9 & 282677.1 & 282657.6 \\
\hline
\end{tabular}

Note. Model A is the unconditional means model and Model B is the unconditional growth model. Model $\mathrm{C}$ includes $\mathrm{RoC} * \mathrm{RoI}$ as a predictor of initial status only. Model $\mathrm{D}$ evaluates the effects of RoC*RoI on initial status, controlling for the effects of Age on initial status. These models predict Stable Risk in New Zealand parolees as a function of days since first DRAOR assessment (at level-1) and various combinations of RoC*RoI and Age (at level-2). All level-2 predictors are grand mean centered.

$* * * p<.001$. 
Figure 4. Graph of prototypical change trajectories using the Stable Risk models.

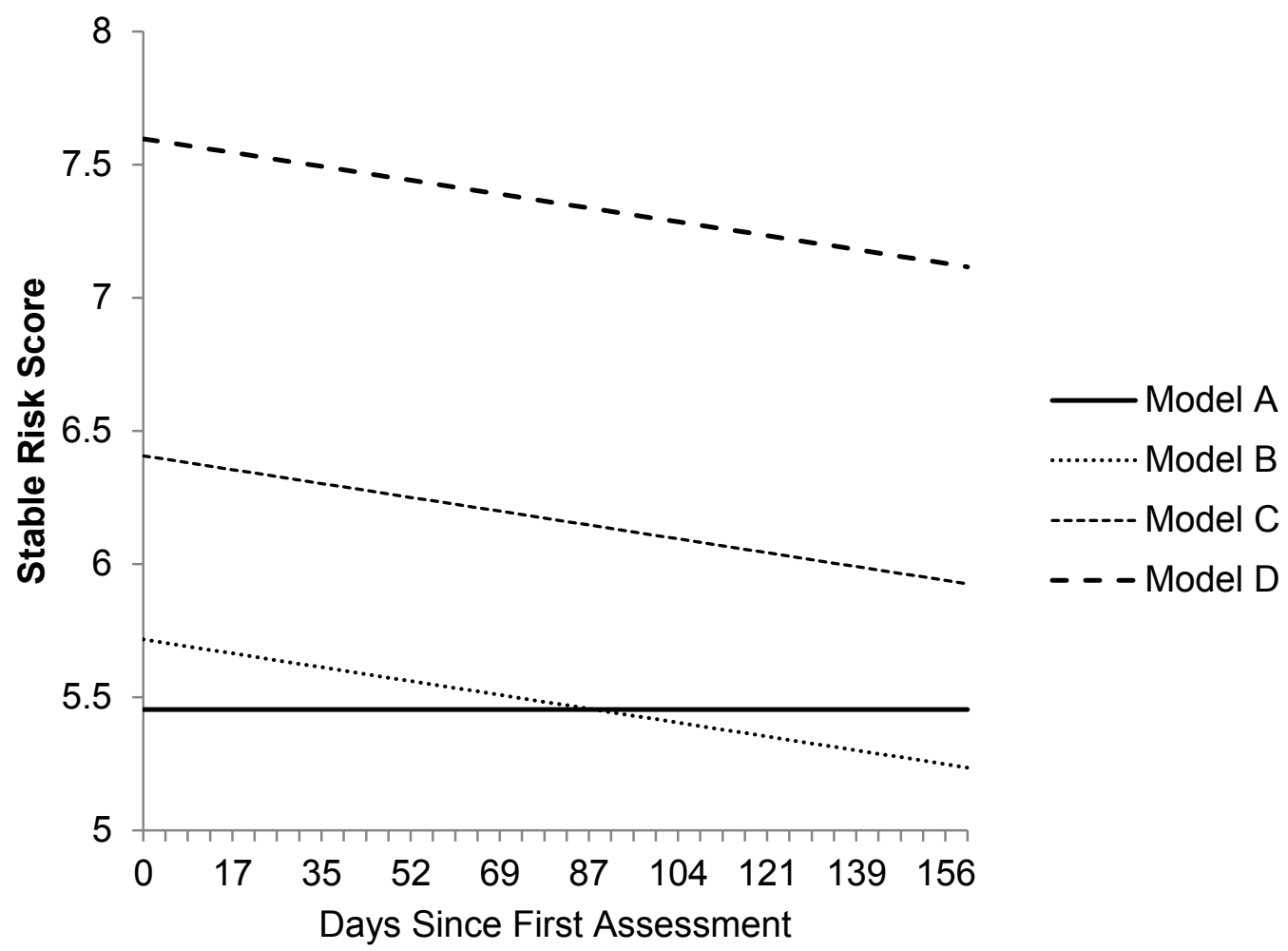

Acute risk. Table 26 presents the unconditional means model (Model A), unconditional growth model (Model B), and potential level-2 between-offender models (Models C and D), while the results of model fitting are displayed in Table 27. The unconditional means model produces an average score of 4.84 in Acute Risk across all assessments and offenders. The variance components are significantly different from zero, meaning there is some variation at both level-1 $\left(\sigma^{2} \varepsilon=2.40, p<.001\right)$ and level-2 $\left(\sigma^{2}{ }_{0}=3.87, p<.001\right)$ to be explained. The intra-class correlation coefficient of .77 indicates that $77 \%$ of the total variation in Acute Risk lies between offenders.

The unconditional growth model shows how Acute Risk score differs between parolees and changes over time. Pseudo $\mathrm{R}^{2} \varepsilon$ indicates the proportion of within-offender variation explained by time is $34 \%$. The grand mean of Acute Risk score across all 
occasions and offenders is 5.31. The change coefficient of -..01 indicates that Acute Risk score decreases by .01 on average per day after first assessment. The level-1 residual variance indicates an average scatter of 1.59 of offender's observed outcome values around their own true change trajectory. The unpredicted variability is 4.43 in true initial status and .0001 in true rate of change. As such, level-2 predictors should be added to potentially explain this variation in individual initial status and rate of change.

Model $\mathrm{C}$ adds $\mathrm{RoC}^{*} \mathrm{RoI}$ to both the intercept and slope to explain different initial values at first assessment and different rates of change across assessments in Acute Risk. The fixed effects for initial status and rate of change intercepts do not change with the addition of this level-2 variable. As static risk increases, the estimated mean of Acute Risk score for the parolee population increases by .73. The cross-level interaction $\left(\gamma_{11}\right)$ is indicative of a moderation effect in which there are different slopes of Acute Risk for different levels of static risk. As static risk increases, the rate of change of Acute Risk decreases by .002. Model D includes Age as a control predictor of initial status only. Once the effects of Age are removed, the intercept for initial status remains the same while the fixed effect for RoC*RoI increases by $57 \%$. The fixed effect for Age indicates a differential of .02 in Acute Risk score for a one-unit difference in Age controlling for the effect of Static Risk. Both of these models suggest that parolees with higher static risk at release initially have higher Acute Risk scores at first assessment and a faster decline in scores across assessments. The magnitude of the early differential in Acute Risk scores is higher after Age is controlled.

A graph of fitted trajectories for a prototypical offender using Models A through $\mathrm{D}$ is presented in Figure 5. Deviance-based hypothesis tests indicate that Model C is a 
better fit than Model B $\left(\chi^{2}(2)=22.95, p<.001\right)$, and Model D is a better fit than Model C $\left(\chi^{2}(1)=25.74, p<.001\right)$. Model $\mathrm{D}$ demonstrates the best fit overall, as evidenced by the lowest AIC and BIC statistics, therefore it is selected as a tentative final model.

However, the Pseudo $\mathrm{R}_{\mathrm{y}, \mathrm{y}}^{2}$ statistics indicate that the amount of variation in Protective Factors accounted for by the predictors in each of these models is only $2 \%$ to $6 \%$. The residual variances in Models $\mathrm{C}$ and $\mathrm{D}$ indicate that there are considerable inter-individual differences which remain unexplained by the models' predictors. The level-2 variance in initial status only decreased by .01\% from Model B to Model C and .01\% from Model C to Model D. The statistical significance of these variance components highlights the potentially explainable residual variation remaining in initial status of Acute Risk. The level-2 variance in rate of change did not change despite the addition of Static Risk to the slope. Thus, Model D is tentative until further model building can be explored with additional within- and between-offender predictors. 
Table 26

Taxonomy of Multilevel Models for Change Fitted to Acute Risk Data

Level-1/Level-2 Specification

Model Level-1 Model Level-2 Model Composite Model

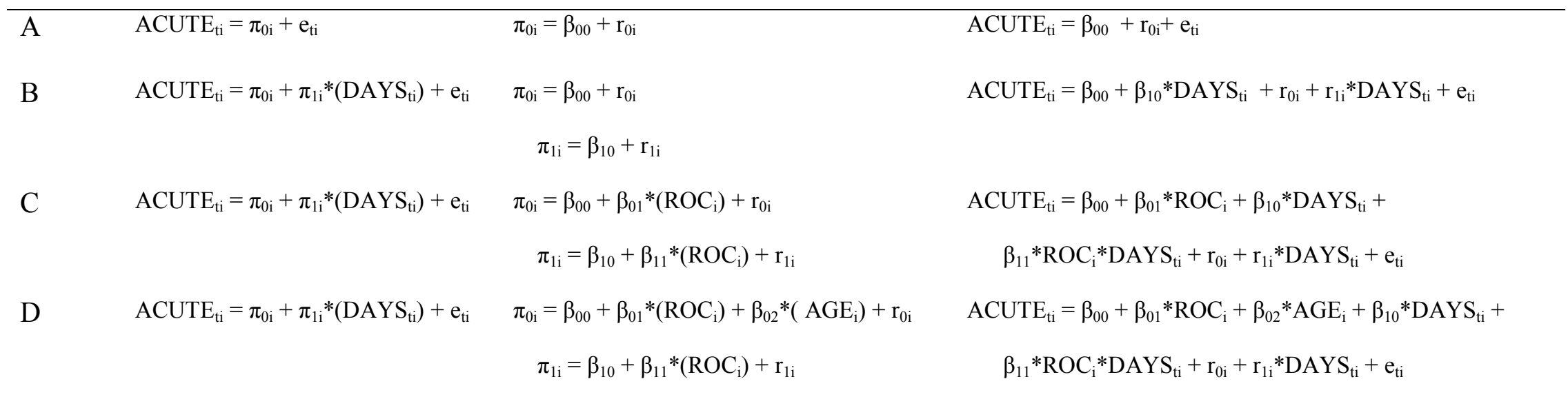

Note. These models predict Acute Risk in all New Zealand parolees as a function of days since first assessment (at level-1) and various combinations of RoC*RoI and Age (at level-2). Models C and D enter the level-2 predictors in grand mean centered form. 
Table 27

Results of Fitting a Taxonomy of Multilevel Models for Change for Acute Risk $(N=$ 3317)

\begin{tabular}{|c|c|c|c|c|c|c|}
\hline & & Parameter & Model A & Model B & Model C & Model D \\
\hline Fixed E & fects & & & Estimate & tandard Err & \\
\hline Initial & Intercept & $\gamma_{00}$ & $4.84 * * *$ & $5.31 * * *$ & $5.31 * * *$ & $5.31 * * *$ \\
\hline status, & & 800 & $(.04)$ & $(.04)$ & $(.04)$ & $(.04)$ \\
\hline$\pi_{0 \mathrm{i}}$ & RoC*RoI & $\gamma_{01}$ & & & $.73 * * *$ & $1.15 * * *$ \\
\hline & & 101 & & & $(.15)$ & $(.18)$ \\
\hline & Age & $\gamma_{02}$ & & & & $.02 * *$ \\
\hline & & & & & & $(.003)$ \\
\hline Rate of & Intercept & $\gamma_{10}$ & & $-.01 * * *$ & $-.01 * * *$ & $-.001 * * *$ \\
\hline change, & & & & $(.0002)$ & $(.0002)$ & $(.0002)$ \\
\hline$\pi_{1 \mathrm{i}}$ & RoC*RoI & $\gamma_{11}$ & & & $-.002 *$ & $-.002 * * *$ \\
\hline & & & & & $(.001)$ & $(.001)$ \\
\hline
\end{tabular}

\begin{tabular}{lllllll}
\hline Variance Components & & \multicolumn{4}{l}{ Estimate (Standard Error) } \\
\hline Level 1 & Within- & $\sigma^{2}{ }_{\varepsilon}$ & $2.40^{* * *}$ & $1.59^{* * *}$ & $1.21 * * *$ & $1.59 * * *$ \\
& person & & $(1.55)$ & $(1.26)$ & $(1.10)$ & $(1.26)$ \\
Level 2 & In initial & $\sigma^{2}{ }_{0}$ & $3.87^{* * *}$ & $4.43^{* * *}$ & $4.39^{* * *}$ & $4.37^{* * *}$ \\
& status & & $(1.97)$ & $(2.10)$ & $(2.10)$ & $(2.09)$ \\
& $\begin{array}{l}\text { In rate of } \\
\text { change }\end{array}$ & $\sigma^{2}{ }_{1}$ & & $.0001^{* * *}$ & $.0001^{* * *}$ & $.0001^{* * *}$ \\
& & & $(.01)$ & $(.01)$ & $(.01)$
\end{tabular}

\begin{tabular}{cllll}
\hline \multicolumn{5}{l}{ Pseudo $\mathrm{R}^{2}$ Statistics and Goodness-of-fit } \\
\hline $\mathrm{R}^{2}{ }_{\mathrm{y}, \mathrm{y}}$ & - & .02 & .05 & .06 \\
$\mathrm{R}^{2} \varepsilon$ & - & .34 & .34 & .34 \\
$\mathrm{R}^{2}{ }_{0}$ & - & - & .01 & .01 \\
$\mathrm{R}^{2}$ & - & - & .00 & .00 \\
Deviance & 328537.6 & 302234.2 & 302211.2 & 302185.5 \\
AIC & 328543.6 & 302240.1 & 302227.2 & 302203.5 \\
BIC & 328571.6 & 302302.2 & 302301.9 & 302287.6 \\
\hline
\end{tabular}

Note. Model A is the unconditional means model and Model B is the unconditional growth model. Model $\mathrm{C}$ includes $\mathrm{RoC} * \mathrm{RoI}$ as a predictor of both initial status and change. Model D evaluates the effects of RoC*RoI on initial status and rate of change, controlling for the effects of Age on initial status only. These models predict Acute Risk in New Zealand parolees as a function of days since first DRAOR assessment (at level-1) and various combinations of $\mathrm{RoC}^{*} \mathrm{RoI}$ and Age (at level-2). All level-2 predictors are grand mean centered.

$* p<.05,{ }^{* *}, p<.01 * * * p<.001$. 
Figure 5. Graph of prototypical change trajectories using the Acute Risk models.

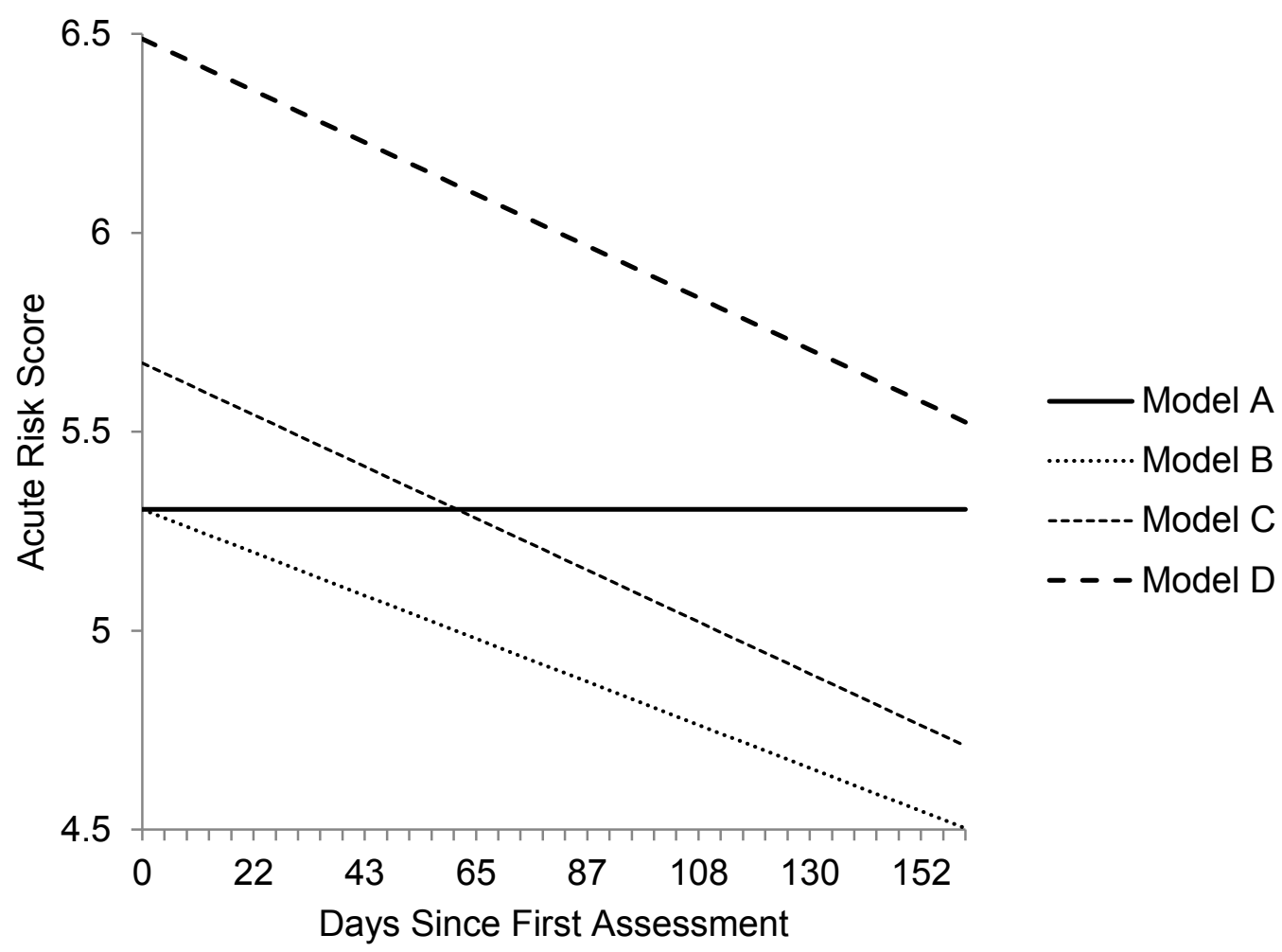

Protective factors. The unconditional means model (Model A), unconditional growth model (Model B), and potential level-2 between-offender models (Models C and D) are displayed in Table 28 and results displayed in Table 29. Across all assessments and offenders, the grand mean of Protective Factors is 7.08 in the unconditional means model. Both the within-offender $\left(\sigma^{2} \varepsilon=1.95, p<.001\right)$ and between-offender $\left(\sigma^{2}{ }_{0}=5.98\right.$, $p<.001)$ variances are significantly different from zero. Thus there is some variation in parolees' Protective Factors at first assessment and over the course of assessments that could potentially be explained by adding predictors at level- 1 and level-2. The majority of the variance in Protective Factors occurs between offenders at level-2 $(\mathrm{ICC}=.75)$.

The unconditional growth model provides an estimated starting point of 6.77 in Protective Factor score and a slope of .004 of the population average change trajectory. 
The proportion of within-offender variation explained by time based on Pseudo $\mathrm{R}^{2} \varepsilon$ is $38 \%$. Each offender's data has a scatter of 1.20 around his or her own linear change trajectory in Protective Factor scores. There is also between-offender variability in initial status at first assessment $\left(\sigma^{2}{ }_{0}=6.13\right)$ and rate of change across assessments $\left(\sigma^{2}{ }_{1}=.0001\right)$. From Models A and B it can be concluded that there is potentially predictable variation in Protective Factors. The unconditional means model suggests more between-offender variation than within-offender variation. The unconditional growth model suggests that some of the within-offender variation is attributable to linear time and that there is between-offender variation in both true initial status and true rate of change that level-2 predictors might explain.

Model $\mathrm{C}$ adds $\mathrm{RoC} * \mathrm{RoI}$ as a level-2 predictor of initial status of Protective Factor scores. The fixed effects for initial status and rate of change intercepts remain the same with the addition of this between-offender predictor. As static risk increases, the estimated mean of Protective Factor score for the parolee population decreases by 1.38. Model D adds the number of days between the first assessment and follow-up end date as a control predictor of initial status only. Once controlling for the effects of the number of days between first assessment and follow-up end, the intercept for initial status remains the same while the fixed effect for $\mathrm{RoC} * \mathrm{RoI}$ increases by $12 \%$. The fixed effect for days between first assessment and follow-up describes a differential of .001 in Protective Factor score for a one-unit difference in the number of days, controlling for the effect of Static Risk. Both Models C and D indicate that parolees with higher static risk at release initially have lower Protective Factor scores at first assessment. The magnitude of the 
early differential in Protective Factor scores is lower after the number of days between first assessment and follow-up is controlled.

Figure 6 displays fitted trajectories of the Protective Factor scores for a prototypical offender using Models A through D. ${ }^{15}$ The deviance-based hypothesis test for Model C indicates improved fit over Model B $\left(\chi^{2}(1)=64.25, p<.001\right)$. Likewise, Model D demonstrates better fit than Model C $\left(\chi^{2}(1)=8.30, p=.004\right)$. The AIC statistic suggests Model D has the best fit, while the BIC statistic suggests that Model C demonstrates a better fit. The Pseudo $\mathrm{R}_{\mathrm{y}, \mathrm{y}}^{2}$ statistics indicate that the amount of variation in Protective Factors accounted for by the predictors in these models is only $1 \%$ to $6 \%$. The residual variation indicates there is variation in Protective Factors left unexplained by both of these models' predictors. The level-2 variance in initial status only decreased by $2 \%$ from Model B to Model C and .2\% from Model C to Model D. Both of these variance components are still statistically significant, therefore there remains potentially explainable residual variation in initial status of Protective Factors. No predictors were added to the slopes, therefore the level-2 variance in rate of change did not change. Due to the minimal change in residual variation, the BIC statistics, and in the interest of parsimony, Model C is selected as a tentative final model over Model D until additional level-1 and level-2 predictors can be explored. Within-offender time-varying predictors are needed, as evidenced by the statistically significant level-1 within-person variance components for Models C and D being identical to Model B.

\footnotetext{
${ }^{15}$ Prototypical changes trajectories were developed based on the mean number of assessments $(M=37.32)$ and the mean number of days since the first assessments $(M=161.73)$. These were divided to obtain a mean number of days between assessments $(M=4.33)$. Protective factor scores were then calculated for 37 assessments for an offender with 4.33 days between each assessment. The mean RoC*RoI score $(M=$ .503 ) and mean number of days between first assessment and follow-up end were imputed into Models $\mathrm{C}$ and D.
} 
Table 28

Taxonomy of Multilevel Models for Change Fitted to Protective Factors Data

\section{Level-1/Level-2 Specification}

\begin{tabular}{|c|c|c|c|}
\hline Model & Level-1 Model & Level-2 Model & Composite Model \\
\hline $\mathrm{A}$ & PROTECT $_{\mathrm{ti}}=\pi_{0 \mathrm{i}}+\mathrm{e}_{\mathrm{ti}}$ & $\pi_{0 \mathrm{i}}=\beta_{00}+\mathrm{r}_{0 \mathrm{i}}$ & PROTECT $_{\mathrm{ti}}=\beta_{00}+\mathrm{r}_{0 \mathrm{i}}+\mathrm{e}_{\mathrm{ti}}$ \\
\hline B & PROTECT $_{\mathrm{ti}}=\pi_{0 \mathrm{i}}+\pi_{1 \mathrm{i}} *\left(\mathrm{DAYS}_{\mathrm{ti}}\right)+\mathrm{e}_{\mathrm{ti}}$ & $\begin{array}{l}\pi_{0 \mathrm{i}}=\beta_{00}+\mathrm{r}_{0 \mathrm{i}} \\
\pi_{1 \mathrm{i}}=\beta_{10}+\mathrm{r}_{1 \mathrm{i}}\end{array}$ & PROTECT $_{\mathrm{ti}}=\beta_{00}+\beta_{10} * \mathrm{DAYS}_{\mathrm{ti}}+\mathrm{r}_{0 \mathrm{i}}+\mathrm{r}_{1 \mathrm{i}} * \mathrm{DAYS}_{\mathrm{ti}}+\mathrm{e}_{\mathrm{ti}}$ \\
\hline $\mathrm{C}$ & PROTECT $_{t i}=\pi_{0 \mathrm{i}}+\pi_{1 \mathrm{i}} *\left(\right.$ DAYS $\left._{\mathrm{ti}}\right)+\mathrm{e}_{\mathrm{ti}}$ & $\begin{array}{l}\pi_{0 \mathrm{i}}=\beta_{00}+\beta_{01} *\left(\mathrm{ROC}_{\mathrm{i}}\right)+\mathrm{r}_{0 \mathrm{i}} \\
\pi_{1 \mathrm{i}}=\beta_{10}+\mathrm{r}_{1 \mathrm{i}}\end{array}$ & $\begin{array}{l}\text { PROTECT }_{\mathrm{ti}}=\beta_{00}+\beta_{01} * \text { ROC }_{\mathrm{i}}+\beta_{10} * \text { DAYS }_{\mathrm{ti}}+\mathrm{r}_{0 \mathrm{i}}+ \\
\mathrm{r}_{1 \mathrm{i}} * \mathrm{DAYS}_{\mathrm{ti}}+\mathrm{e}_{\mathrm{ti}}\end{array}$ \\
\hline $\mathrm{D}$ & PROTECT $_{\mathrm{ti}}=\pi_{0 \mathrm{i}}+\pi_{1 \mathrm{i}} *\left(\right.$ DAYS $\left._{\mathrm{ti}}\right)+\mathrm{e}_{\mathrm{ti}}$ & $\begin{array}{l}\pi_{0 \mathrm{i}}=\beta_{00}+\beta_{01} *\left(\mathrm{ROC}_{\mathrm{i}}\right)+\beta_{02} *\left(\mathrm{FAFO}_{\mathrm{i}}\right)+\mathrm{r}_{0 \mathrm{i}} \\
\pi_{1 \mathrm{i}}=\beta_{10}+\mathrm{r}_{1 \mathrm{i}}\end{array}$ & $\begin{array}{l}\text { PROTECT }_{\mathrm{ti}}=\beta_{00}+\beta_{01} * \text { ROC }_{\mathrm{i}}+\beta_{02} * \mathrm{FAFO}_{\mathrm{i}}+\beta_{10} * \mathrm{DAYS}_{\mathrm{ti}} \\
+\mathrm{r}_{0 \mathrm{i}}+\mathrm{r}_{1 \mathrm{i}} * \mathrm{DAYS}_{\mathrm{ti}}+\mathrm{e}_{\mathrm{ti}}\end{array}$ \\
\hline
\end{tabular}

Note. These models predict Protective Factor in all New Zealand parolees as a function of days since first assessment (at level-1) and various combinations of RoC*RoI and number of days between first assessment and follow-up end date (at level-2). Models C and D enter the level-2 predictors in grand mean centred form. 
Table 29

Results of Fitting a Taxonomy of Multilevel Models for Change for Protective Factors (N

$=3317)$

\begin{tabular}{|c|c|c|c|c|c|c|}
\hline \multirow{2}{*}{\multicolumn{2}{|c|}{ Fixed Effects }} & Parameter & Model A & Model B & Model C & Model D \\
\hline & & & & \multicolumn{3}{|c|}{ Estimate (Standard Error) } \\
\hline \multirow{4}{*}{$\begin{array}{l}\text { Initial } \\
\text { status, } \\
\pi_{0 \mathrm{i}}\end{array}$} & Intercept & $\gamma_{00}$ & $7.08 * * *$ & $6.77 * * *$ & $6.77 * * *$ & $6.77 * * *$ \\
\hline & & & $(.04)$ & $(.04)$ & $(.04)$ & $(.04)$ \\
\hline & RoC*RoI & $\gamma_{01}$ & & & $\begin{array}{l}-1.38 * * * \\
(.17)\end{array}$ & $\begin{array}{l}-1.53 * * * \\
(.18)\end{array}$ \\
\hline & $\begin{array}{l}\text { First } \\
\text { assessment } \\
\text { to follow-up }\end{array}$ & $\gamma_{02}$ & & & & $\begin{array}{l}-.001 * * \\
(.0002)\end{array}$ \\
\hline \multirow{3}{*}{$\begin{array}{l}\text { Rate of } \\
\text { change, } \\
\pi_{1 \mathrm{i}}\end{array}$} & Intercept & $\gamma_{10}$ & & $.004 * * *$ & $.004 * * *$ & $.004 * * *$ \\
\hline & & & & $(.0002)$ & $(.0002)$ & $(.0002)$ \\
\hline & & & & & & \\
\hline \multicolumn{3}{|c|}{ Variance Components } & \multicolumn{4}{|c|}{ Estimate (Standard Error) } \\
\hline \multirow[t]{2}{*}{ Level 1} & Within- & $\sigma^{2} \varepsilon$ & $1.95 * * *$ & $1.20 * * *$ & $1.20 * * *$ & $1.20 * * *$ \\
\hline & person & & $(1.40)$ & $(1.10)$ & $(1.10)$ & $(1.10)$ \\
\hline \multirow[t]{4}{*}{ Level 2} & In initial & $\sigma_{0}^{2}$ & $5.98 * * *$ & $6.13 * * *$ & $6.00 * * *$ & $5.99 * * *$ \\
\hline & status & & $(2.45)$ & $(2.48)$ & $(2.45)$ & $(2.45)$ \\
\hline & In rate of & $\sigma_{1}^{2}$ & & $.0001 * * *$ & $.0001 * * *$ & $.0001 * * *$ \\
\hline & change & & & $(.01)$ & $(.01)$ & $(.01)$ \\
\hline \multicolumn{7}{|c|}{ Pseudo $\mathrm{R}^{2}$ Statistics and Goodness-of-fit } \\
\hline & $\mathrm{R}_{\mathrm{y}, \mathrm{y}}^{2}$ & & - & .01 & .06 & .04 \\
\hline & $\mathrm{R}^{2} \varepsilon$ & & - & .38 & .38 & .38 \\
\hline & $\mathrm{R}^{2}{ }_{0}$ & & - & - & .02 & .02 \\
\hline & Deviance & & 313163.7 & 281295.1 & 281230.8 & 281222.5 \\
\hline & AIC & & 313167.7 & 281301.1 & 281244.8 & 281238.5 \\
\hline & $\mathrm{BIC}$ & & 313197.7 & 281363.1 & 281310.2 & 281313.2 \\
\hline
\end{tabular}

Note. Model A is the unconditional means model and Model B is the unconditional growth model. Model $\mathrm{C}$ includes RoC*RoI as a predictor of initial status. Model D evaluates the effects of RoC*RoI on initial status, controlling for the effects of the number of days between first assessment and follow-up date on initial status. These models predict Protective Factor in New Zealand parolees as a function of days since first DRAOR assessment (at level-1) and various combinations of RoC*RoI and number of days between first assessment and follow-up date (at level-2). All level-2 predictors are grand mean centered.

$* p<.05, * * p<.01, * * * p<.001$. 
Figure 6. Graph of prototypical change trajectories using the Protective Factor models.

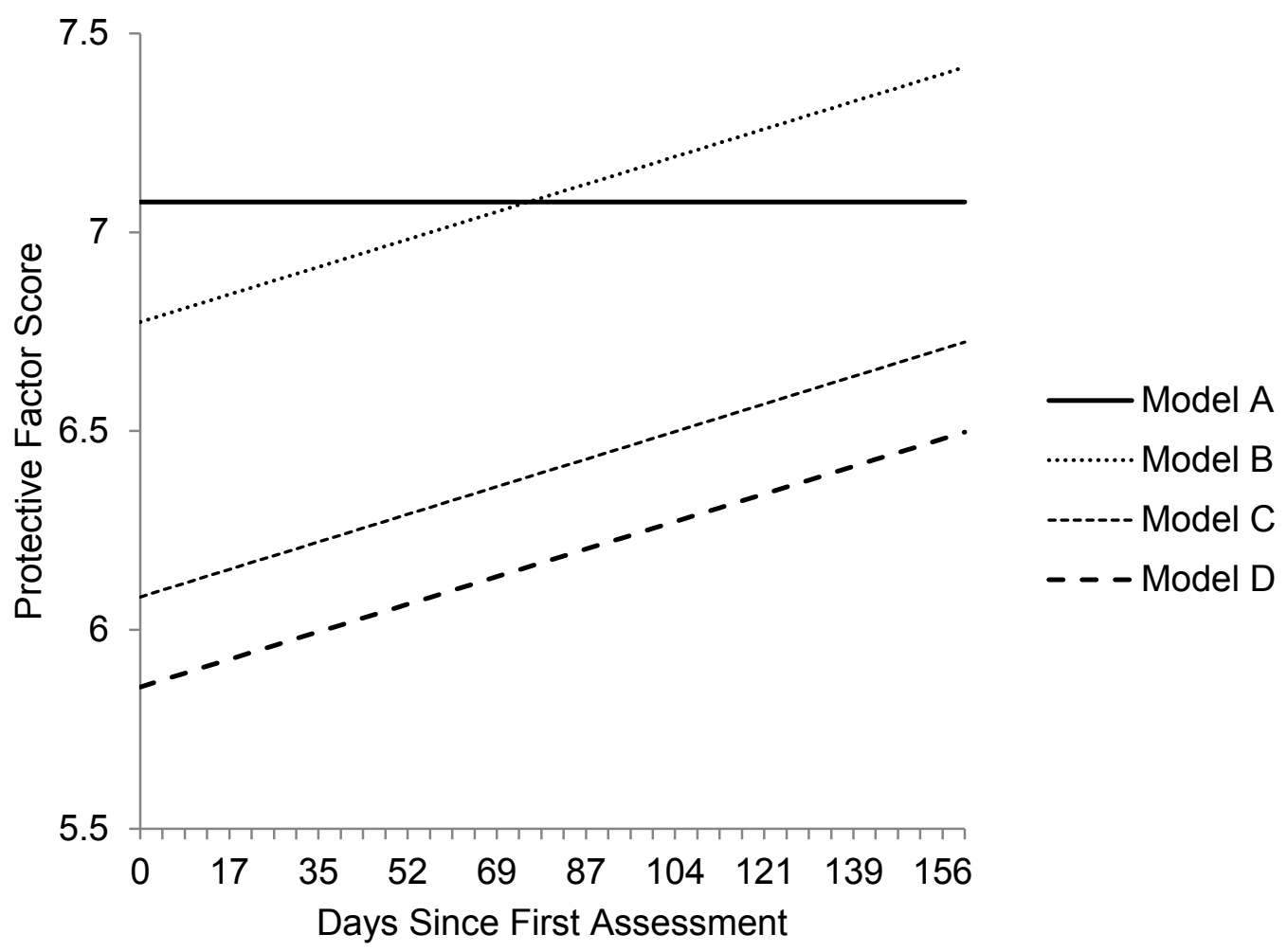

Recidivists versus non-recidivists hypothesis testing. The goal of the previous section was model building in the interest of examining how Stable Risk, Acute Risk, and Protective Factors differ both within- and between-offenders at first assessment and over the course of assessments. This section focuses on hypothesis testing. Three additional level-2 models were created to examine whether dynamic risk and protective factors changed over time between offenders who were reconvicted versus those who were not. This analysis focuses on inter-individual change as opposed to intra-individual change. Offenders were assigned a level-2 variable categorizing them as recidivists or nonrecidivists depending on whether they incurred a reconviction during the study period. As with the previous growth models, offenders were only included if they had a minimum of 
two assessments following their baseline third assessment. Assessments following the first reconviction were excluded from the analysis.

This analysis tests if the average growth trends over time differ for offenders who are reconvicted versus those who are not. Different scores are expected to be observed at the starting period and/or in changes in score over time between groups. This approach explains variance across offenders in change over time, and determines whether changes in scores yield different outcomes. This analysis was first performed on any reconvictions, then repeated for offenders with criminal reconvictions versus those with no criminal reconvictions. The multilevel models are displayed in Appendix H. No additional predictors were entered into the models to provide uncontrolled answers to the research questions. Only deviance statistics are presented for each of the models, as model building was not the goal of this analysis.

All reconvictions. Given that the level-2 variable was simultaneously added to the intercept and slope, multiparameter hypothesis testing was required. The results of this hypothesis testing demonstrate differences between groups overall for Stable Risk $\left(\chi^{2}(2)=18.69, p<.001\right)$, Acute Risk $\left(\chi^{2}(2)=8.03, p=.02\right)$, and Protective Factors $\left(\chi^{2}(2)\right.$ $=12.82, p<.001)$. This overall significance permits the examination of the slopes and intercepts individually. Table 30 presents the results of hypothesis testing for each outcome variable. 
Table 30

Results of Hypothesis Testing for All Reconvictions $(N=3317)$

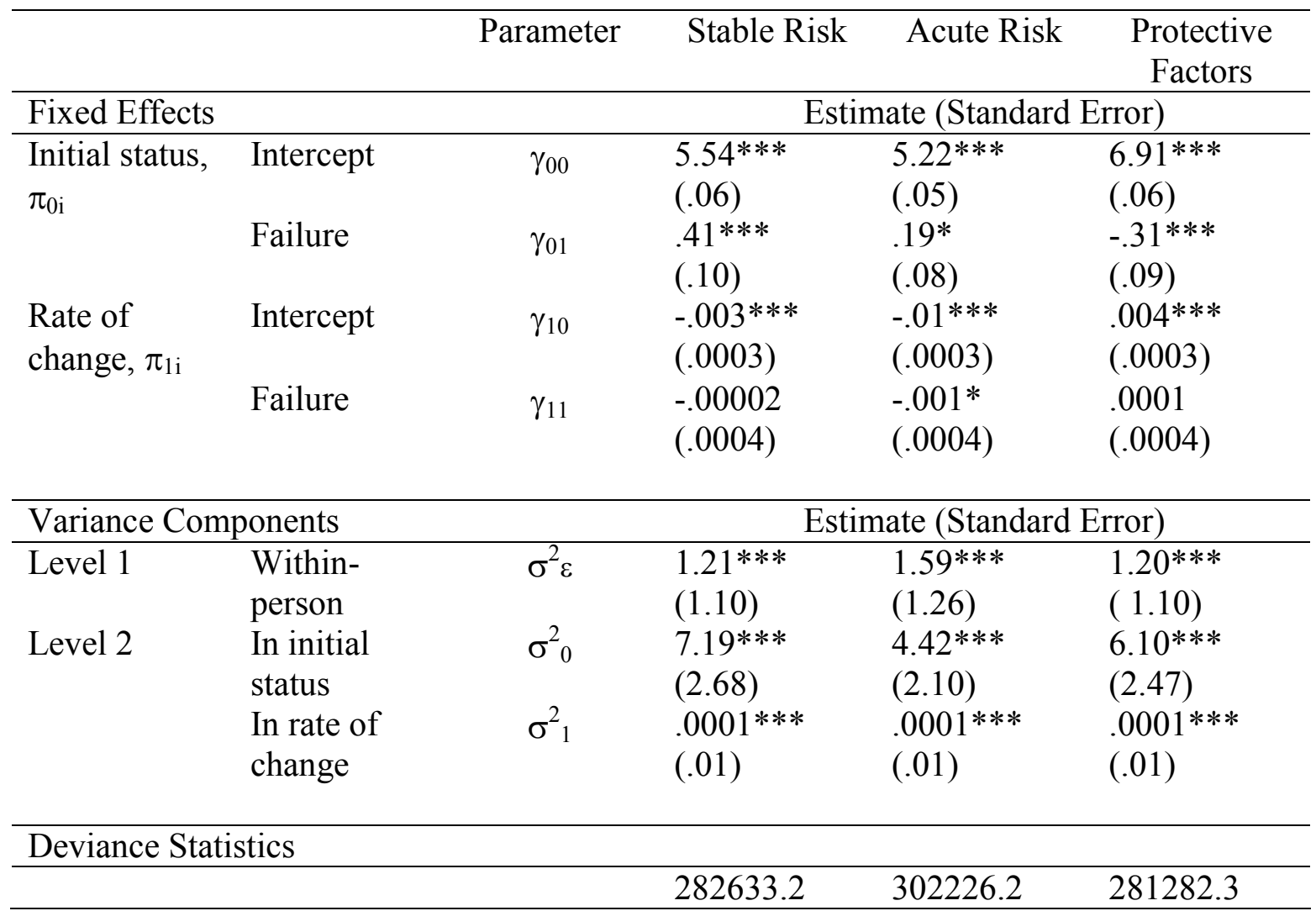

Note. These models predict Stable Risk, Acute Risk and Protective Factors in New Zealand parolees as a function of days since first DRAOR assessment (at level-1) and a reconviction variable representing recidivists and non-recidivists (at level-2). The level-2 variable is uncentered.

$* p<.05, * * p<.01, * * * p<.001$.

Beginning with Stable Risk, the level-2 model provides an estimated starting point of 5.54 in Stable Risk Score and a decline of .003 in score per day after first assessment. Recidivists and non-recidivists were significantly different from one another in first assessment scores, with recidivists initially having a score .41 higher than nonrecidivists. The rate of change in Stable Risk score did not differ significantly between recidivists and non-recidivists. The results from this model indicate that although recidivists and non-recidivists differed in Stable Risk score at first assessment, their rate 
of change in Stable Risk score did not differ across assessments. Figure 7 displays fitted trajectories for two prototypical offenders - one recidivist and one non-recidivist. ${ }^{16}$

Figure 7. Graph of prototypical change trajectories in Stable Risk using all reconvictions.

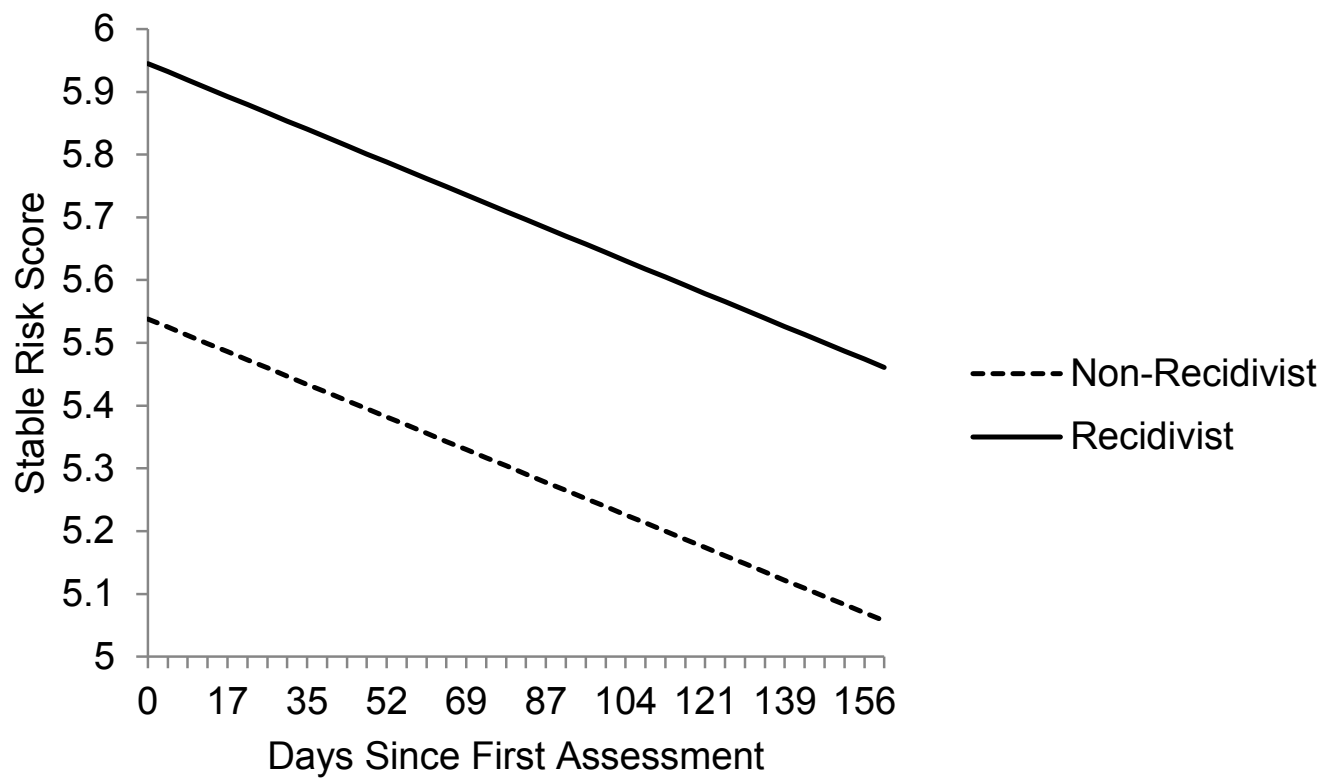

The level-2 model for Acute Risk provides an estimated starting point of 5.22 in Acute Risk score at first assessment and a decline of .01 in scores across assessments. Recidivists had significantly higher Acute Risk than non-recidivists with an initial score .19 higher at first assessment. The rate of change across assessments differed depending on whether the offender was reconvicted. The rate of change for recidivists was .001 lower than non-recidivists. The results of this model indicate that not only do recidivists and non-recidivists have different initial Acute Risk scores, but also their rate of change in these scores differs between groups over time. Prototypical change trajectories in Acute Risk for recidivists and non-recidivists are presented in Figure 8.

\footnotetext{
${ }^{16}$ Prototypical changes trajectories were developed based on the mean number of assessments $(M=37.32)$ and the mean number of days since the first assessments $(M=161.73)$. These were divided to obtain a mean number of days between assessments $(M=4.33)$. Stable risk scores were then calculated for 37 assessments for a recidivist and 37 assessments for a non-recidivists with 4.33 days between each assessment.
} 
Figure 8. Graph of prototypical change trajectories in Acute Risk using all reconvictions.

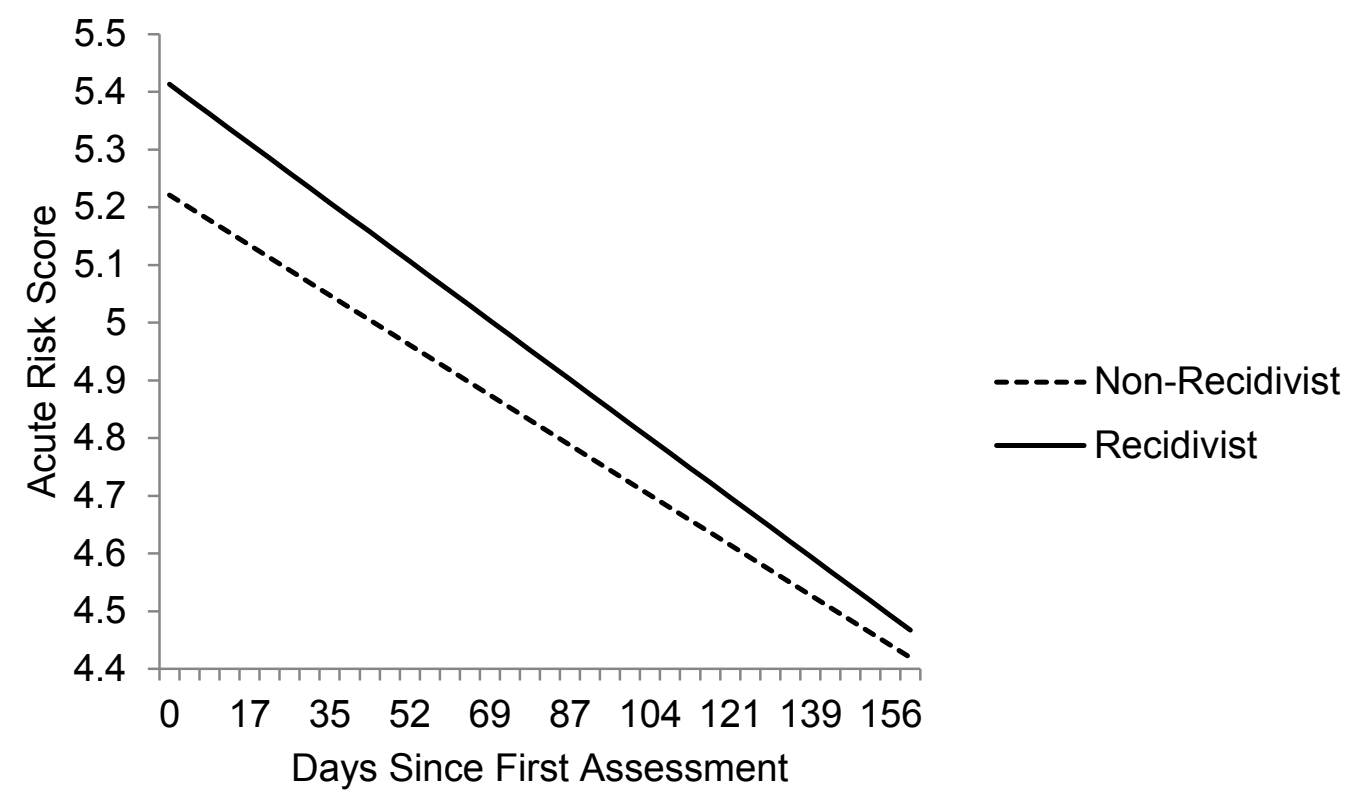

Lastly, the estimated starting score of Protective Factors at first assessment is 6.91 with an increase of .004 in score per day across the assessment period. Recidivists had a significantly lower Protective Factor score than non-recidivists by .31 at first assessment. As can be seen in the prototypical change trajectories displayed in Figure 9, the rate of change in Protective Factor scores did not differ across assessments between those offenders who were reconvicted compared to those who were not. In sum, recidivists and non-recidivists differed in their initial Protective Factor scores at first assessment, but their rate of change in these scores did not differ across assessments. 
Figure 9. Graph of prototypical change trajectories in Protective Factors using all reconvictions.

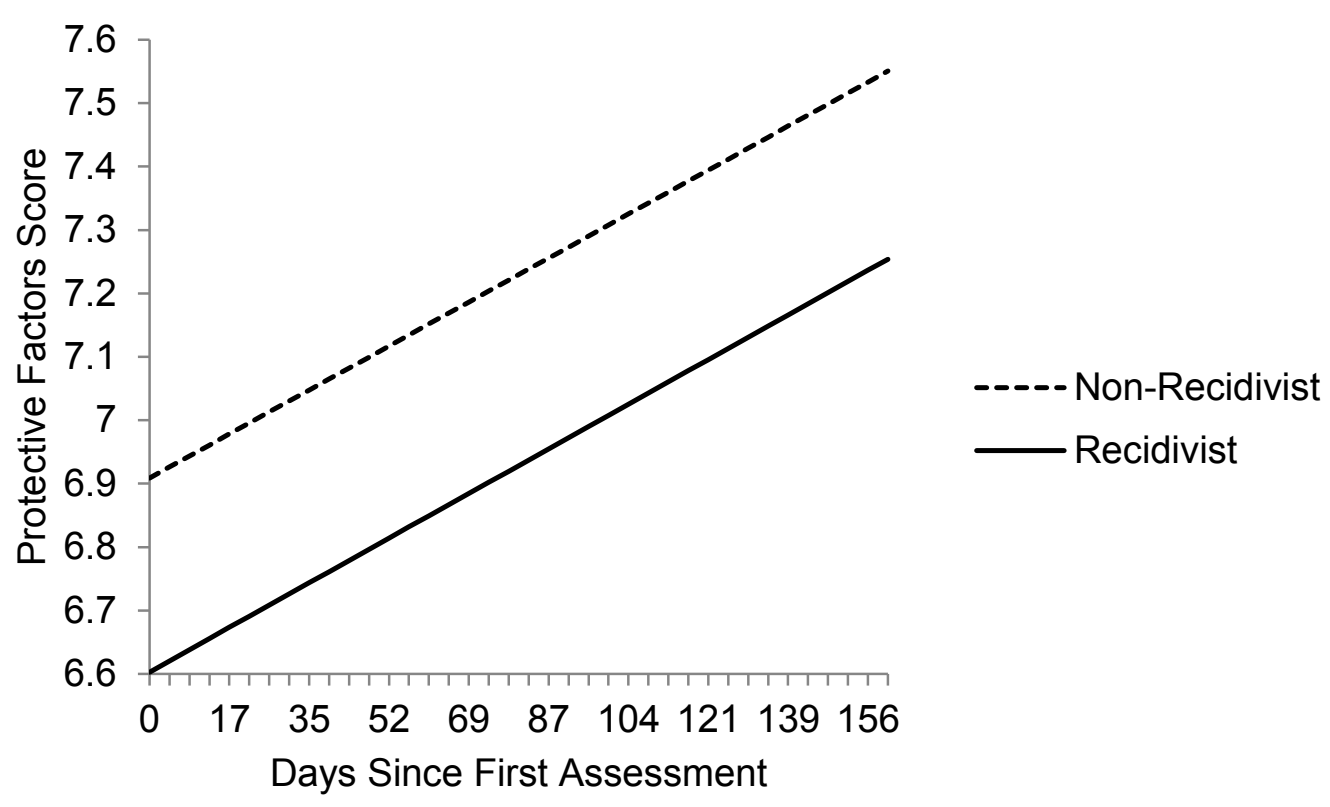

Criminal reconvictions. The results from the models with reconviction level-2 variables entered suggest that recidivists differ from non-recidivists at first assessment in their Stable Risk, Acute Risk, and Protective Factor scores. While recidivists also show a faster decline in Acute Risk than non-recidivists, they do not differ in rate of change of Stable Risk or Protective Factors. The preceding analysis was repeated for criminal reconvictions and the same pattern of findings emerged. Multiparameter hypothesis testing confirmed an overall significant effect for Stable Risk $\left(\chi^{2}(2)=12.46, p<.01\right)$, Acute Risk $\left(\chi^{2}(2)=9.50, p<.01\right)$, and Protective Factors $\left(\chi^{2}(2)=7.82, p=.02\right)$. Differences between groups were then examined at initial status and rate of change. Results are presented in Table 31. 
Table 31

Results of Hypothesis Testing for Criminal Reconvictions $(N=3317)$

\begin{tabular}{|c|c|c|c|c|c|}
\hline & & Parameter & Stable Risk & Acute Risk & $\begin{array}{c}\text { Protective } \\
\text { Factors }\end{array}$ \\
\hline Fixed Effects & & & \multicolumn{3}{|c|}{ Estimate (Standard Error) } \\
\hline \multirow[t]{2}{*}{$\begin{array}{l}\text { Initial status, } \\
\pi_{0 \mathrm{i}}\end{array}$} & Intercept & $\gamma_{00}$ & $\begin{array}{l}5.61 * * * \\
(.06)\end{array}$ & $\begin{array}{l}5.24 * * * \\
(.05)\end{array}$ & $\begin{array}{l}6.85 * * * \\
(.05)\end{array}$ \\
\hline & $\begin{array}{l}\text { Criminal } \\
\text { reconviction }\end{array}$ & $\gamma_{01}$ & $\begin{array}{l}.36^{* * * *} \\
(.10)\end{array}$ & $\begin{array}{l}.20^{*} \\
(.08)\end{array}$ & $\begin{array}{l}-.25 * * * \\
(.10)\end{array}$ \\
\hline \multirow[t]{2}{*}{$\begin{array}{l}\text { Rate of } \\
\text { change, } \pi_{1 \mathrm{i}}\end{array}$} & Intercept & $\gamma_{10}$ & $\begin{array}{l}-.003 * * * \\
(.0003)\end{array}$ & $\begin{array}{l}-.01 * * * \\
(.0003)\end{array}$ & $\begin{array}{l}.004 * * * \\
(.0003)\end{array}$ \\
\hline & $\begin{array}{l}\text { Criminal } \\
\text { reconviction }\end{array}$ & $\gamma_{11}$ & $\begin{array}{l}-.0003 \\
(.0004)\end{array}$ & $\begin{array}{l}-.001 * \\
(.001)\end{array}$ & $\begin{array}{l}-.00001 \\
(.001)\end{array}$ \\
\hline \multicolumn{3}{|c|}{ Variance Components } & \multicolumn{3}{|c|}{ Estimate (Standard Error) } \\
\hline Level 1 & $\begin{array}{l}\text { Within- } \\
\text { person }\end{array}$ & $\sigma^{2} \varepsilon$ & $\begin{array}{l}1.21^{* * *} \\
(1.10)\end{array}$ & $\begin{array}{l}1.59 * * * \\
(1.26)\end{array}$ & $\begin{array}{l}1.20 * * * \\
(1.10)\end{array}$ \\
\hline \multirow[t]{2}{*}{ Level 2} & $\begin{array}{l}\text { In initial } \\
\text { status }\end{array}$ & $\sigma_{0}^{2}$ & $\begin{array}{l}7.20 * * * \\
(2.68)\end{array}$ & $\begin{array}{l}4.42 * * * \\
(2.10)\end{array}$ & $\begin{array}{l}6.11 * * * \\
(2.47)\end{array}$ \\
\hline & $\begin{array}{l}\text { In rate of } \\
\text { change }\end{array}$ & $\sigma_{1}^{2}$ & $\begin{array}{l}.0001^{* * * *} \\
(.01)\end{array}$ & $\begin{array}{l}.0001^{* * * *} \\
(.01)\end{array}$ & $\begin{array}{l}.0001 * * * \\
(.01)\end{array}$ \\
\hline \multicolumn{6}{|c|}{ Deviance Statistics } \\
\hline & & & 282639.4 & 302224.7 & 281287.2 \\
\hline
\end{tabular}

Note. These models predict Stable Risk, Acute Risk and Protective Factors in New Zealand parolees as a function of days since first DRAOR assessment (at level-1) and a criminal reconviction variable representing recidivists and non-recidivists (at level-2). The level-2 variable is uncentered. $* p<.05, * * p<.01, * * * p<.001$.

The level-2 model for Stable Risk provides an estimated starting point of 5.61 at first assessment and a decline of .003 in score per day thereafter. As with all reconvictions, criminal recidivists initially had a score higher than non-recidivists (by .36) but did not differ in their rate of change. The estimated score at first assessment for Acute Risk was 5.24, with a decline of .01 per day over the course of assessments. Mirroring the findings for all reconvictions as well, criminal recidivists had a significantly higher score by .20 at first assessment, and showed a faster rate of change $\left(\gamma_{11}=-.001\right)$ than non-recidivists. Lastly, the estimated starting score of Protective 
Factors at first assessment was 6.85 with an increase of .004 in score per day across the assessment period. The rate of change in Protective Factor scores did not differ between recidivists and non-recidivists, but offenders with a criminal reconviction had a significantly lower score by .25 at first assessment than those without. Prototypical change trajectories are displayed graphically in Figure 10 for Stable Risk, Figure 11 for Acute Risk, and Figure 12 for Protective Factors.

Figure 10. Graph of prototypical change trajectories in Stable Risk using criminal reconvictions.

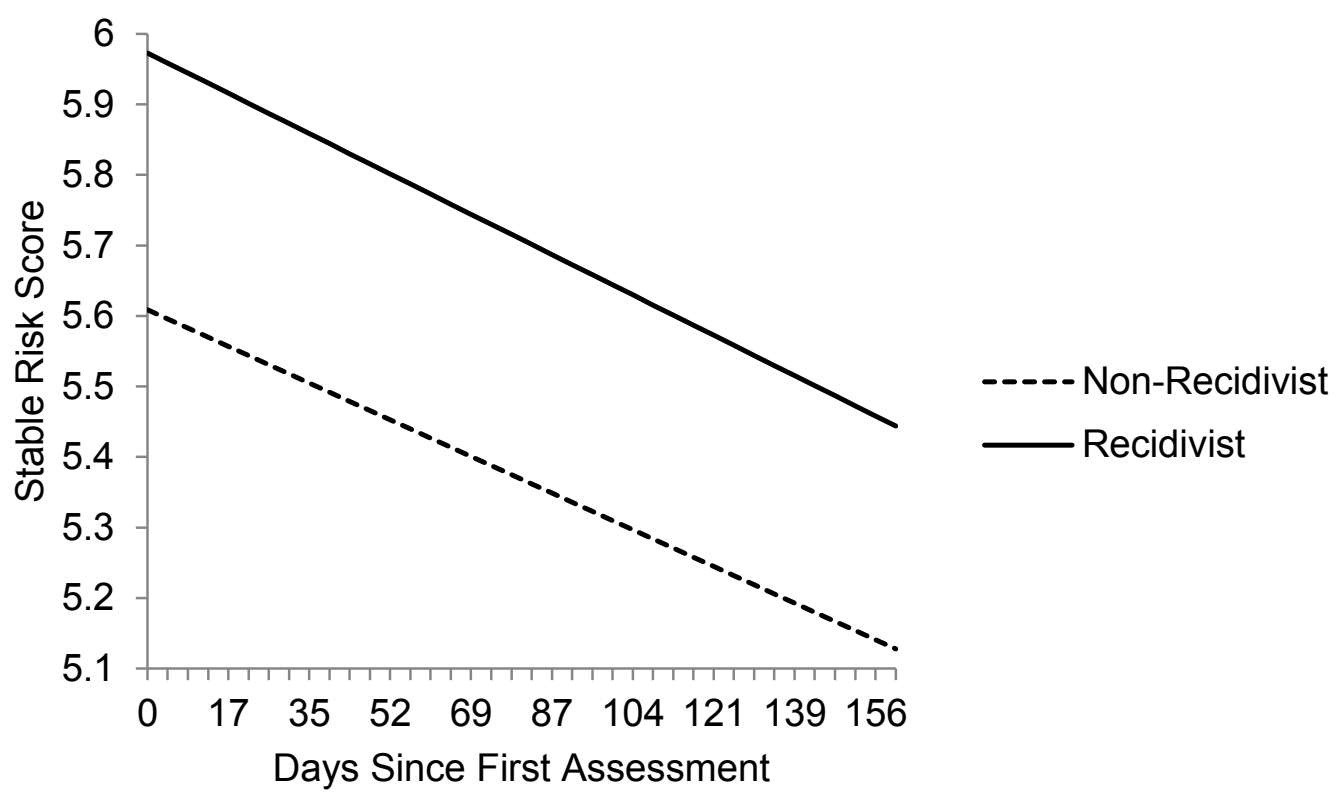


Figure 11. Graph of prototypical change trajectories in Acute Risk using criminal reconvictions.

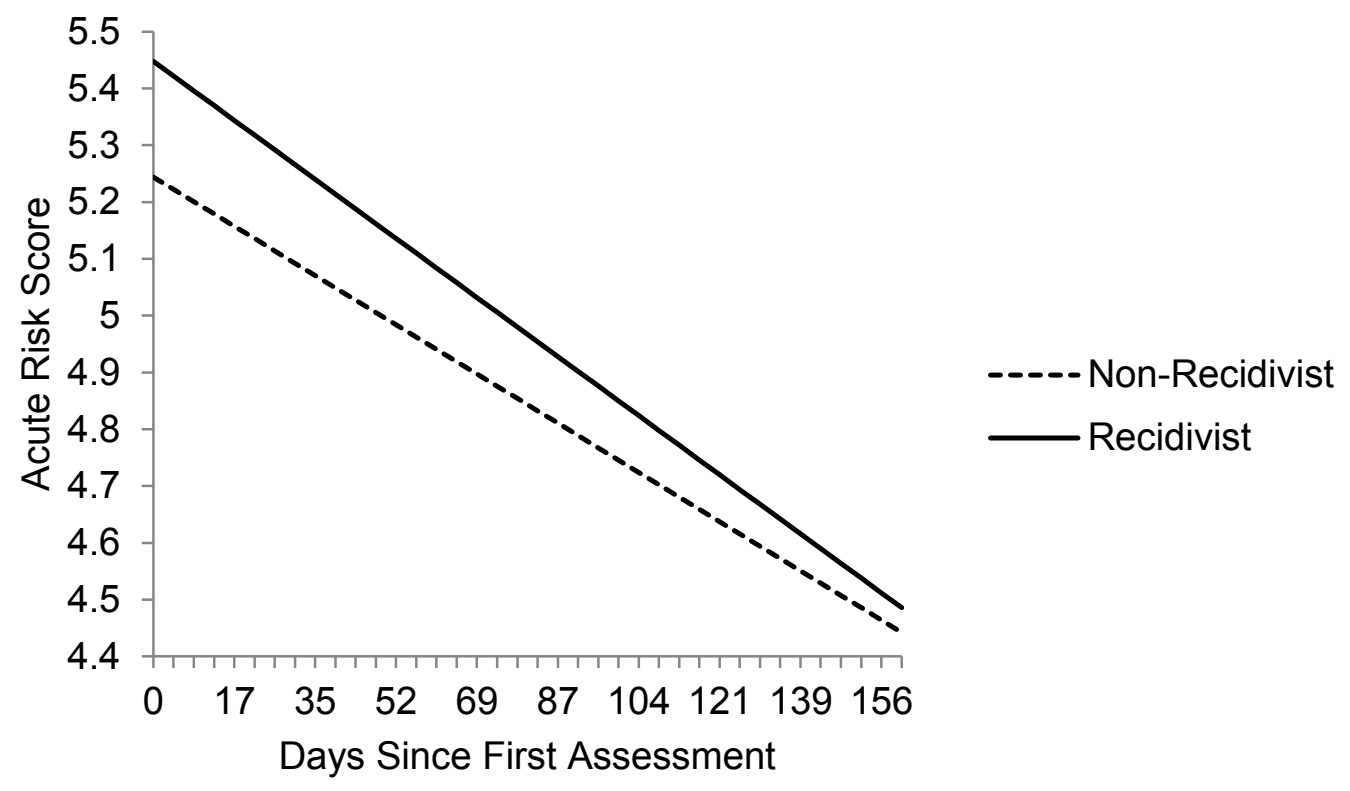

Figure 12. Graph of prototypical change trajectories in Protective Factors using criminal reconvictions.

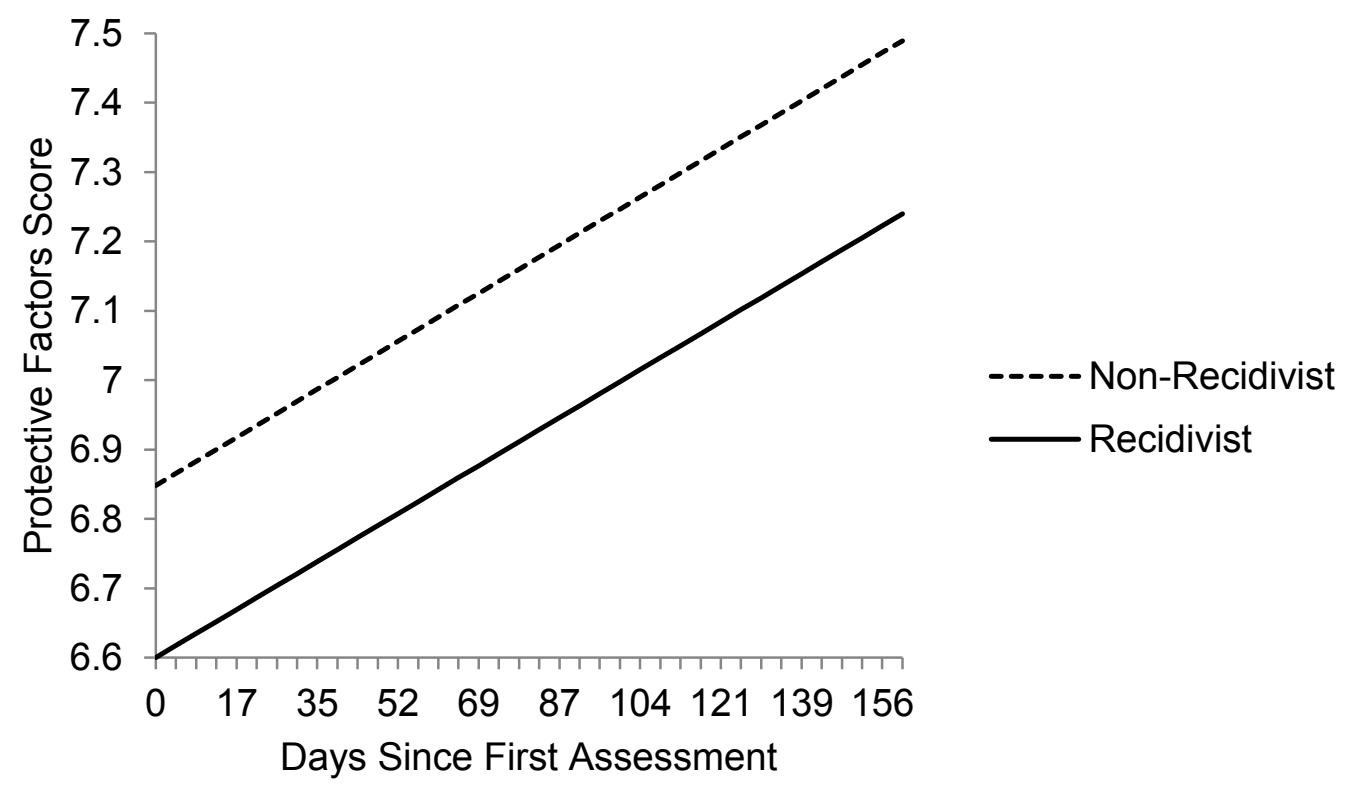


Summary: Part 5. Table 32 presents a narrative summary of Part 5 findings

relating to change in dynamic risk over a period of assessment and reassessment.

Table 32

Summary of Part 5 Results

\begin{tabular}{|c|c|c|}
\hline Analysis & $\begin{array}{l}\text { Outcome } \\
\text { Variable }\end{array}$ & Main Findings \\
\hline \multirow[t]{3}{*}{$\begin{array}{l}\text { Model } \\
\text { Building }\end{array}$} & Stable Risk & $\begin{array}{l}\text { The Stable Risk score across all occasions and offenders } \\
\text { was } 5.72 \text { at first assessment and decreased over time. } \\
\text { Controlling for age, static risk predicted between-offender } \\
\text { variability in scores at first assessment. }\end{array}$ \\
\hline & Acute Risk & $\begin{array}{l}\text { The average Acute Risk score was } 5.31 \text { at first assessment } \\
\text { and decreased over time. Controlling for age, static risk } \\
\text { predicted between-offender variability in scores at first } \\
\text { assessment. Static risk was also a between-offender } \\
\text { predictor of rate of change in Acute Risk. }\end{array}$ \\
\hline & $\begin{array}{l}\text { Protective } \\
\text { Factors }\end{array}$ & $\begin{array}{l}\text { The average Protective Factor score was } 6.77 \text { at first } \\
\text { assessment and increased over time. Static risk predicted } \\
\text { between-offender variability in scores at first assessment. }\end{array}$ \\
\hline \multirow[t]{3}{*}{$\begin{array}{l}\text { Hypothesis } \\
\text { Testing }\end{array}$} & Stable Risk & $\begin{array}{l}\text { Recidivists showed Stable Risk scores .41 higher than } \\
\text { non-recidivists at first assessment. Recidivists did not } \\
\text { show a different pattern of change in Stable Risk across } \\
\text { time compared to non-recidivists. }\end{array}$ \\
\hline & Acute Risk & $\begin{array}{l}\text { Recidivists had Acute Risk scores } .19 \text { higher than non- } \\
\text { recidivists at first assessment. The Acute Risk scores of } \\
\text { recidivists decreased at a faster rate over time than non- } \\
\text { recidivists. }\end{array}$ \\
\hline & $\begin{array}{l}\text { Protective } \\
\text { Factors }\end{array}$ & $\begin{array}{l}\text { The Protective Factors score of recidivists was } .31 \text { lower } \\
\text { than non-recidivists at first assessment. Their rates of } \\
\text { change in Protective Factors did not differ over time. }\end{array}$ \\
\hline
\end{tabular}

\footnotetext{
${ }^{1}$ These results were consistent with the categorization of recidivists and non-recidivists based on both all reconvictions and criminal reconvictions. The differences in scores at first assessment presented are based on all reconvictions.
} 


\section{Part 6. Timing of DRAOR Assessments}

Hypothesis 7. The DRAOR will significantly predict reconvictions from the first assessment, with higher accuracy just prior to reoffending.

A two-part approach was employed to predict when the DRAOR is predictive of recidivism, and whether the DRAOR is useful at every time point. The first approach is descriptive. Additional multilevel models were developed for Stable Risk, Acute Risk, and Protective Factors, replacing TIME with dummy codes at level-1 that flag when the assessments took place. Working backwards, assessments in the last month before recidivism or censoring were identified as 0 , the month prior were coded 1 , the month before that were coded 2 and so forth. The intercept indicates the average dynamic risk scores just prior to recidivism or censoring, to determine whether they increase or decrease going backwards in time. The dummy codes express the difference in intercept compared to the baseline final month before recidivism or censoring. Essentially, instead of one time variable entered at level-1 and represented as a slope in the model, categorical codes are entered at each month to represent a series of averages across months. These analyses describe the general trend over time (i.e., the slope), and the dummy codes indicate where the peaks and plateaus are. Adding the reconvictions variable at level-2 determines if these peaks and plateaus are different for recidivists compared to non-recidivists. Appendix I displays the multilevel models tested for each of the outcome variables.

Treating time in this manner is described in Singer and Willett (2003) of dividing continuous time into contiguous epochs, in the context of Cox regression. Examining reverse causation like this approach was also used with regression analysis in a recent 
study of therapist-patient alliances by Crits-Christoph and colleagues (2011). Given that one of the advantages of multilevel modelling is that time can be treated flexibly, this approach can be extended to the current analysis. Recentering time in this manner will produce an interpretive advantage to address different questions: Are recidivists peaking in dynamic risk scores a few months prior to recidivism, or immediately before? At what point do they plateau, remaining non-significantly different from the last assessments before recidivism? Locating the trajectories' anchor at the endpoint means the intercept parameters assess effects in the month prior to recidivism or censoring. Singer and Willett (2003) suggest endpoint centering when final status is of special concern. Looking at monthly scores is a coarser temporal predictor but the benefit is that it does not place any constraints on the functional form of the time-varying effect.

The second approach to answering at what point the DRAOR becomes predictive of recidivism involves regular Cox regression survival analysis. This procedure is useful for modelling the specified time to a specified event, based upon the values of given covariates. In this case, Stable Risk, Acute Risk, and Protective Factors are used as covariates to predict the event of reconvictions or criminal reconvictions. A total of 12 models were computed with Cox regression survival analysis using assessments taken month by month, each time replacing the predictor from a different month. The first analysis used the first month following parole start date of assessments of dynamic risk and Protective Factors to predict recidivism. The second analysis used the assessments of the second month to predict recidivism, and so forth. Effect sizes are computed for each analysis and compared to determine if later assessments have larger effect sizes than earlier assessments. The follow-up length is controlled for in the analysis. Grouping 
assessments into these larger groups of time makes the metric more meaningful, aiding in modelling over time and making practical conclusions. The analysis "pretends" that there are assessments earlier in time than there really are, but this allows for a direct comparison of whether later month assessments are predicting recidivism more strongly than earlier assessments. Models for each monthly scores will be compared using a measure of effect size $\left(\mathrm{R}^{2}\right)$ recommended by Allison $(1995$, as cited in Tabachnick \& Fidell, 2007). This measure represents the relative association between survival time and the covariates tested, compared to the baseline model with no covariates entered.

Growth models. The 12 months prior to follow-up end were included in the growth models as the number of assessments per month began to taper off at 13 months. In addition, it was important to model a substantial time period (i.e., 12 months instead of 6 months) to accurately capture changes in dynamic risk over time. This resulted in a sample size of 58306 DRAOR assessments at level-1 and 3240 offenders at level-2. Dummy codes were created to identify Months 2 through 12 prior to recidivism or censoring, while the intercept represents Month 1. Each monthly coefficient represents the magnitude difference between that month and the intercept (i.e., average score in the month prior to follow-up end). The results of multilevel modelling using monthly variables are displayed in Table 33 for Stable Risk, Table 34 for Acute Risk, and Table 35 for Protective Factors. For each outcome variable, an unconditional growth model is first presented which includes only the monthly variables at level-1. The second model adds all reconvictions to each of the level-2 intercepts and slopes to compare recidivists to non-recidivists. The final model replaces all reconvictions with criminal reconvictions at each month. 
Table 33

Results of Multilevel Modelling of Stable Risk Using Monthly Time Variables $(N=3240)$

\begin{tabular}{|c|c|c|c|c|c|c|c|}
\hline \multirow{2}{*}{\multicolumn{2}{|c|}{ Fixed Effects }} & \multicolumn{2}{|c|}{$\begin{array}{l}\text { Unconditional } \\
\text { Growth }\end{array}$} & \multicolumn{2}{|c|}{ All Reconvictions } & \multicolumn{2}{|c|}{$\begin{array}{c}\text { Criminal } \\
\text { reconvictions }\end{array}$} \\
\hline & & Estimate & $\mathrm{SE}$ & Estimate & $\mathrm{SE}$ & Estimate & $\mathrm{SE}$ \\
\hline \multirow{2}{*}{$\begin{array}{l}\text { Initial } \\
\text { status, } \pi_{0 \mathrm{i}}\end{array}$} & Intercept $\left(\gamma_{00}\right)$ & $5.08 * * *$ & .05 & $4.90 * * *$ & .07 & $4.98 * * *$ & .06 \\
\hline & Failure $\left(\gamma_{01}\right)$ & & & $.42 * * *$ & .10 & $.37 * * *$ & .11 \\
\hline \multirow{22}{*}{$\begin{array}{l}\text { Rate of } \\
\text { change, } \\
\pi_{1 \mathrm{i}}\end{array}$} & Mo. $2\left(\gamma_{10}\right)$ & -.001 & .02 & -.02 & .03 & -.003 & .02 \\
\hline & Failure $\left(\gamma_{11}\right)$ & & & .05 & .04 & .01 & .04 \\
\hline & Mo. $3\left(\gamma_{20}\right)$ & $.10 * * *$ & .02 & $.10 * * *$ & .03 & $.13 * * *$ & .03 \\
\hline & Failure $\left(\gamma_{21}\right)$ & & & .01 & .04 & -.08 & .05 \\
\hline & Mo. $4\left(\gamma_{30}\right)$ & $.20 * * *$ & .02 & $.20 * * *$ & .03 & $.24 * * *$ & .03 \\
\hline & Failure $\left(\gamma_{31}\right)$ & & & .01 & .05 & $-.11 *$ & .05 \\
\hline & Mo. $5\left(\gamma_{40}\right)$ & $.23 * * *$ & .02 & $.23 * * *$ & .03 & $.28 * * *$ & .03 \\
\hline & Failure $\left(\gamma_{41}\right)$ & & & .01 & .05 & $-.14 * *$ & .05 \\
\hline & Mo. $6\left(\gamma_{50}\right)$ & $.38 * * *$ & .03 & $.36 * * *$ & .03 & $.43 * * *$ & .03 \\
\hline & Failure $\left(\gamma_{51}\right)$ & & & .05 & .05 & $-.18 * *$ & .05 \\
\hline & Mo. $7\left(\gamma_{60}\right)$ & $.42 * * *$ & .03 & $.40 * * *$ & .04 & $.48 * * *$ & .03 \\
\hline & Failure $\left(\gamma_{61}\right)$ & & & .04 & .05 & $-.20 * * *$ & .06 \\
\hline & Mo. $8\left(\gamma_{70}\right)$ & $.48 * * *$ & .03 & $.45 * * *$ & .04 & $.55 * * *$ & .03 \\
\hline & Failure $\left(\gamma_{71}\right)$ & & & .06 & .05 & $-.24 * * *$ & .06 \\
\hline & Mo. $9\left(\gamma_{80}\right)$ & $.55 * * *$ & .03 & $.52 * * *$ & .04 & $.62 * * *$ & .03 \\
\hline & Failure (81) & & & .07 & .06 & $-.25 * * *$ & .06 \\
\hline & Mo. $10\left(\gamma_{90}\right)$ & $.60 * * *$ & .03 & $.62 * * *$ & .04 & $.67 * * *$ & .03 \\
\hline & Failure $\left(\gamma_{91}\right)$ & & & -.04 & .06 & $-.26 * * *$ & .06 \\
\hline & Mo. $11\left(\gamma_{100}\right)$ & $.75^{* * *}$ & .03 & $.81 * * *$ & .04 & $.84 * * *$ & .04 \\
\hline & Failure $\left(\gamma_{101}\right)$ & & & $-.13 *$ & .06 & $-.30 * * *$ & .0 \\
\hline & Mo. $12\left(\gamma_{110}\right)$ & $.87 * * *$ & .03 & $.89 * * *$ & .04 & $.94 * * *$ & .04 \\
\hline & Failure $\left(\gamma_{111}\right)$ & & & -.05 & .06 & $-.26 * * *$ & .07 \\
\hline \multicolumn{8}{|c|}{ Variance Components } \\
\hline Level 1 & $\begin{array}{l}\text { Within } \\
\text { person }\left(\sigma_{\varepsilon}^{2}\right)\end{array}$ & $1.51 * * *$ & 1.23 & $1.51 * * *$ & 1.23 & $1.51 * * *$ & 1.23 \\
\hline Level 2 & $\begin{array}{l}\text { In initial } \\
\text { status }\left(\sigma_{0}^{2}\right)\end{array}$ & $7.53 * * *$ & 2.75 & $7.49 * * *$ & 2.74 & $7.51 * * *$ & 2.74 \\
\hline \multicolumn{8}{|c|}{ Deviance Statistics } \\
\hline & & \multicolumn{2}{|c|}{206527.6} & \multicolumn{2}{|c|}{206490.2} & \multicolumn{2}{|c|}{206473.5} \\
\hline
\end{tabular}

Note. These models predict Stable Risk in New Zealand parolees as a function of months prior to recidivism or censoring (at level-1) and a reconviction variable representing recidivists and non-recidivists (at level-2). Column 2 displays results for all reconvictions while Column 3 displays results for criminal reconvictions only. The level-2 variable is uncentered.

$* p<.05, * * p<.01, * * * p<.001$. 
Table 34

Results of Multilevel Modelling of Acute Risk Using Monthly Time Variables $(N=3240)$

\begin{tabular}{|c|c|c|c|c|c|c|c|}
\hline \multirow{2}{*}{ Fixed Effect } & & \multicolumn{2}{|c|}{$\begin{array}{l}\text { Unconditional } \\
\text { Growth }\end{array}$} & \multicolumn{2}{|c|}{ All Reconvictions } & \multicolumn{2}{|c|}{$\begin{array}{l}\text { Criminal } \\
\text { reconvictions }\end{array}$} \\
\hline & & Estimate & $\mathrm{SE}$ & Estimate & $\mathrm{SE}$ & Estimate & $\mathrm{SE}$ \\
\hline \multirow{2}{*}{$\begin{array}{l}\text { Initial } \\
\text { status, } \pi_{0 \mathrm{i}}\end{array}$} & Intercept $\left(\gamma_{00}\right)$ & $4.48 * * *$ & .04 & $4.46 * * *$ & .06 & $4.48 * * *$ & .05 \\
\hline & Failure $\left(\gamma_{01}\right)$ & & & .04 & .08 & .02 & .09 \\
\hline \multirow{22}{*}{$\begin{array}{l}\text { Rate of } \\
\text { change, } \\
\pi_{1 \mathrm{i}}\end{array}$} & Mo. $2\left(\gamma_{10}\right)$ & $-.03 * * *$ & .02 & $-.10 * * *$ & .03 & $-.07 * *$ & .03 \\
\hline & Failure $\left(\gamma_{11}\right)$ & & & $.16^{* * *}$ & .04 & $.16^{* * *}$ & .05 \\
\hline & Mo. $3\left(\gamma_{20}\right)$ & $.12 * * *$ & .02 & $.10^{* *}$ & .03 & $.11 * * *$ & .03 \\
\hline & Failure $\left(\gamma_{21}\right)$ & & & .06 & .05 & .05 & .05 \\
\hline & Mo. $4\left(\gamma_{30}\right)$ & $.20 * * *$ & .03 & $.19 * * *$ & .03 & $.20 * * *$ & .03 \\
\hline & Failure $\left(\gamma_{31}\right)$ & & & .02 & .05 & .01 & .06 \\
\hline & Mo. $5\left(\gamma_{40}\right)$ & $.23 * * *$ & .03 & $.22 * * *$ & .03 & $.25 * * *$ & .03 \\
\hline & Failure $\left(\gamma_{41}\right)$ & & & .02 & .05 & -.05 & .06 \\
\hline & Mo. $6\left(\gamma_{50}\right)$ & $.34 * * *$ & .03 & $.39 * * *$ & .04 & $.40 * * *$ & .03 \\
\hline & Failure $\left(\gamma_{51}\right)$ & & & -.10 & .05 & $-.21 * * *$ & .06 \\
\hline & Mo. $7\left(\gamma_{60}\right)$ & $.35 * * *$ & .03 & $.38 * * *$ & .04 & $.40 * * *$ & .03 \\
\hline & Failure $\left(\gamma_{61}\right)$ & & & -.05 & .06 & $-.14 *$ & .06 \\
\hline & Mo. $8\left(\gamma_{70}\right)$ & $.47 * * *$ & .03 & $.44 * * *$ & .04 & $.50 * * *$ & .04 \\
\hline & Failure $\left(\gamma_{71}\right)$ & & & .08 & .06 & -.10 & .06 \\
\hline & Mo. $9\left(\gamma_{80}\right)$ & $.68 * * *$ & .03 & $.62 * * *$ & .04 & $.71 * * *$ & .04 \\
\hline & Failure (81) & & & $.13^{*}$ & .06 & -.08 & .07 \\
\hline & Mo. $10\left(\gamma_{90}\right)$ & $.79 * * *$ & .03 & $.82 * * *$ & .04 & $.83 * * *$ & .04 \\
\hline & Failure $\left(\gamma_{91}\right)$ & & & -.05 & .06 & -.13 & .07 \\
\hline & Mo. $11\left(\gamma_{100}\right)$ & $.97 * * *$ & .03 & $1.00 * * *$ & .04 & $1.04 * * *$ & .04 \\
\hline & Failure $\left(\gamma_{101}\right)$ & & & -.05 & .07 & $-.21 * *$ & .07 \\
\hline & Mo. $12\left(\gamma_{110}\right)$ & $1.09 * * *$ & .03 & $1.10 * * *$ & .05 & $1.13 * * *$ & .04 \\
\hline & Failure $\left(\gamma_{111}\right)$ & & & .06 & .07 & -.13 & .08 \\
\hline \multicolumn{8}{|c|}{ Variance Components } \\
\hline Level 1 & $\begin{array}{l}\text { Within } \\
\text { person }\left(\sigma_{\varepsilon}^{2}\right)\end{array}$ & $1.81 * * *$ & 1.35 & $1.82 * * *$ & 1.35 & $1.81 * * *$ & 1.35 \\
\hline Level 2 & $\begin{array}{l}\text { In initial } \\
\text { status }\left(\sigma_{0}^{2}\right)\end{array}$ & $4.75 * * *$ & 2.18 & $4.75^{* * *}$ & 2.18 & $4.75 * * *$ & 2.18 \\
\hline \multicolumn{8}{|c|}{ Deviance Statistics } \\
\hline & & \multicolumn{2}{|c|}{215423.4} & \multicolumn{2}{|c|}{215380.9} & \multicolumn{2}{|c|}{215365.1} \\
\hline
\end{tabular}

Note. These models predict Acute Risk in New Zealand parolees as a function of months prior to recidivism or censoring (at level-1) and a reconviction variable representing recidivists and non-recidivists (at level-2). Column 2 displays results for all reconvictions while Column 3 displays results for criminal reconvictions only. The level-2 variable is uncentered.

$* p<.05, * * p<.01, * * * p<.001$. 
Table 35

Results of Multilevel Modelling of Protective Factors Using Monthly Time Variables ( $N$ $=3240$ )

\begin{tabular}{|c|c|c|c|c|c|c|c|}
\hline \multirow{2}{*}{ Fixed Effec } & & \multicolumn{2}{|c|}{$\begin{array}{l}\text { Unconditional } \\
\text { Growth }\end{array}$} & \multicolumn{2}{|c|}{ All Reconvictions } & \multicolumn{2}{|c|}{$\begin{array}{c}\text { Criminal } \\
\text { reconvictions }\end{array}$} \\
\hline & & Estimate & $\mathrm{SE}$ & Estimate & $\mathrm{SE}$ & Estimate & $\mathrm{SE}$ \\
\hline \multirow{2}{*}{$\begin{array}{l}\text { Initial } \\
\text { status, } \pi_{0 \mathrm{i}}\end{array}$} & Intercept $\left(\gamma_{00}\right)$ & $7.50 * * *$ & .05 & $7.64 * * *$ & .06 & $7.58 * * *$ & .06 \\
\hline & Failure $\left(\gamma_{01}\right)$ & & & $-.33 * * *$ & .10 & $-.27 * *$ & .10 \\
\hline \multirow{22}{*}{$\begin{array}{l}\text { Rate of } \\
\text { change, } \\
\pi_{1 \mathrm{i}}\end{array}$} & Mo. $2\left(\gamma_{10}\right)$ & $-.04 *$ & .02 & -.02 & .03 & -.04 & .02 \\
\hline & Failure $\left(\gamma_{11}\right)$ & & & -0.05 & .04 & .02 & .04 \\
\hline & Mo. $3\left(\gamma_{20}\right)$ & $-.16 * * *$ & .02 & $-.15 * * *$ & .03 & $-.18 * * *$ & .03 \\
\hline & Failure $\left(\gamma_{21}\right)$ & & & -.01 & .04 & .06 & .05 \\
\hline & Mo. $4\left(\gamma_{30}\right)$ & $-.27 * * *$ & .02 & $-.28 * * *$ & .03 & $-.30 * * *$ & .03 \\
\hline & Failure $\left(\gamma_{31}\right)$ & & & .03 & .04 & $.11^{*}$ & .05 \\
\hline & Mo. $5\left(\gamma_{40}\right)$ & $-.28 * * *$ & .02 & $-.33 * * *$ & .03 & $-.34 * * *$ & .03 \\
\hline & Failure $\left(\gamma_{41}\right)$ & 1. & 2. & $.11 *$ & .05 & $.19 * * *$ & .05 \\
\hline & Mo. $6\left(\gamma_{50}\right)$ & $-.41 * * *$ & .02 & $-.42 * * *$ & .03 & $-.46 * * *$ & .03 \\
\hline & Failure $\left(\gamma_{51}\right)$ & 3. & 4. & .02 & .05 & $.18 * * *$ & .05 \\
\hline & Mo. $7\left(\gamma_{60}\right)$ & $-.47 * * *$ & .03 & $-.48 * * *$ & .03 & $-.55 * * *$ & .03 \\
\hline & Failure $\left(\gamma_{61}\right)$ & & & .02 & .05 & $.25 * * *$ & .05 \\
\hline & Mo. $8\left(\gamma_{70}\right)$ & $-.54 * * *$ & .03 & $-.55 * * *$ & .04 & $-.61 * * *$ & .03 \\
\hline & Failure $\left(\gamma_{71}\right)$ & & & .02 & .05 & $.22 * * *$ & .06 \\
\hline & Mo. $9\left(\gamma_{80}\right)$ & $-.65 * * *$ & .03 & $-.69 * * *$ & .04 & $-.73 * * *$ & .03 \\
\hline & Failure (81) & & & .09 & .06 & $.30 * * *$ & .06 \\
\hline & Mo. $10\left(\gamma_{90}\right)$ & $-.69 * * *$ & .03 & $-.76 * * *$ & .04 & $-.78 * * *$ & .03 \\
\hline & Failure $\left(\gamma_{91}\right)$ & & & $.16^{* *}$ & .06 & $.32 * * *$ & .06 \\
\hline & Mo. $11\left(\gamma_{100}\right)$ & $-.80 * * *$ & .03 & $-.84 * * *$ & .04 & $-.85 * * *$ & .03 \\
\hline & Failure $\left(\gamma_{101}\right)$ & & & .10 & .06 & $.19 * *$ & .06 \\
\hline & Mo. $12\left(\gamma_{110}\right)$ & $-.92 * * *$ & .03 & $-.94 * * *$ & .04 & $-.98 * * *$ & .04 \\
\hline & Failure $\left(\gamma_{111}\right)$ & & & .05 & .06 & $.22 * * *$ & .07 \\
\hline \multicolumn{8}{|c|}{ Variance Components } \\
\hline Level 1 & $\begin{array}{l}\text { Within } \\
\text { person }\left(\sigma_{\varepsilon}^{2}\right)\end{array}$ & $1.41 * * *$ & 1.19 & $1.41 * * *$ & 1.19 & $1.41 * * *$ & 1.19 \\
\hline Level 2 & $\begin{array}{l}\text { In initial } \\
\text { status }\left(\sigma_{0}^{2}\right)\end{array}$ & $6.67 * * *$ & 2.58 & $6.64 * * *$ & 2.58 & $6.65 * * *$ & 2.58 \\
\hline \multicolumn{8}{|c|}{ Deviance Statistics } \\
\hline & & \multicolumn{2}{|c|}{202326.2} & \multicolumn{2}{|c|}{202288.8} & \multicolumn{2}{|c|}{202267.5} \\
\hline
\end{tabular}

Note. These models predict Protective Factors in New Zealand parolees as a function of months prior to recidivism or censoring (at level-1) and a reconviction variable representing recidivists and non-recidivists (at level-2). Column 2 displays results for all reconvictions while Column 3 displays results for criminal reconvictions only. The level-2 variable is uncentered.

$* p<.05, * * p<.01, * * * p<.001$. 
Stable risk. The unconditional growth model for Stable Risk shows an average score across all offenders of 5.08 in the final month prior to recidivism or censoring. With the exception of the non-significant second month prior to follow-up end, all of the months demonstrate an increase in Stable Risk score going backwards in time. This is displayed in Figure 13, which in the interest of clarity reverses the months on the x-axis. For instance, the Month 3 coefficient represents an increase in Stable Risk score of .10 going backwards in time from the third to first month before recidivism or censoring. In other words, the Stable Risk score decreases by .10 from the third month prior to recidivism or censoring to the month prior. Stable Risk appears to plateau in the two months prior to recidivism or censoring, as indicated by very little change in score between Months 2 and 1. The level-1 residual variance shows a scatter of 1.51 of each offender's data around their own linear change trajectory. The level-2 residual variance summarizes between-offender variability of 7.53 in initial status in the baseline month.

Figure 13. Graph of Stable Risk Unconditional Growth Model using monthly variables.

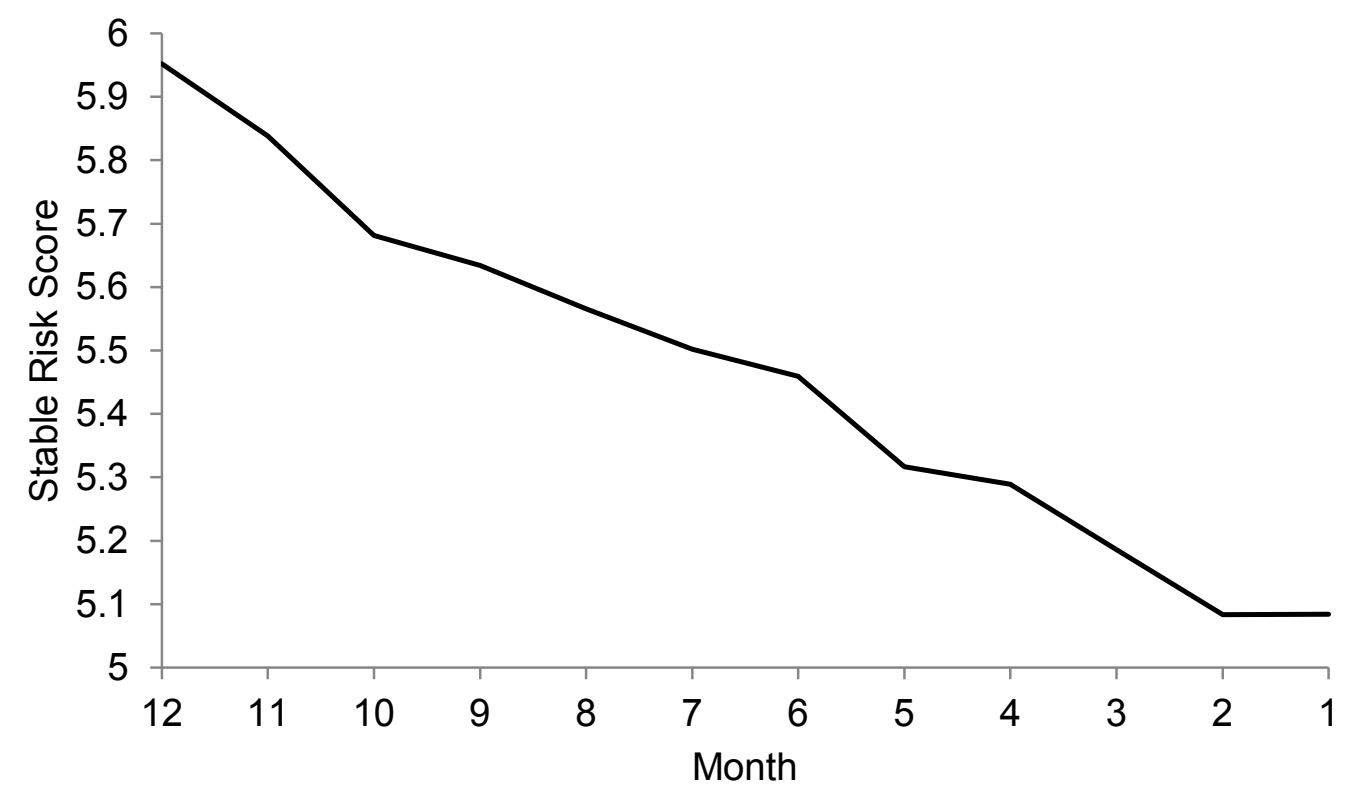


The second model in Table 30 tests whether recidivists with any reconvictions differ from non-recidivists in their initial baseline score or in any of the other months leading up to either recidivism or censoring. As demonstrated in Figure 14, the Stable Risk scores of both recidivists and non-recidivists show a decrease in the 12 months prior to follow-up end. In the earlier months (Months 10-12), non-recidivists actually have slightly higher scores than recidivists, although the only significant difference between groups is found at Month 11. The scores of the groups are almost overlapping in the months leading up to follow-up end. Finally, in the month prior to recidivism, recidivists show a peak in their Stable Risk scores, which is significantly higher than that of their non-recidivist counterparts in the month prior to censoring.

Figure 14. Graph of Stable Risk All Reconvictions Model using monthly variables.

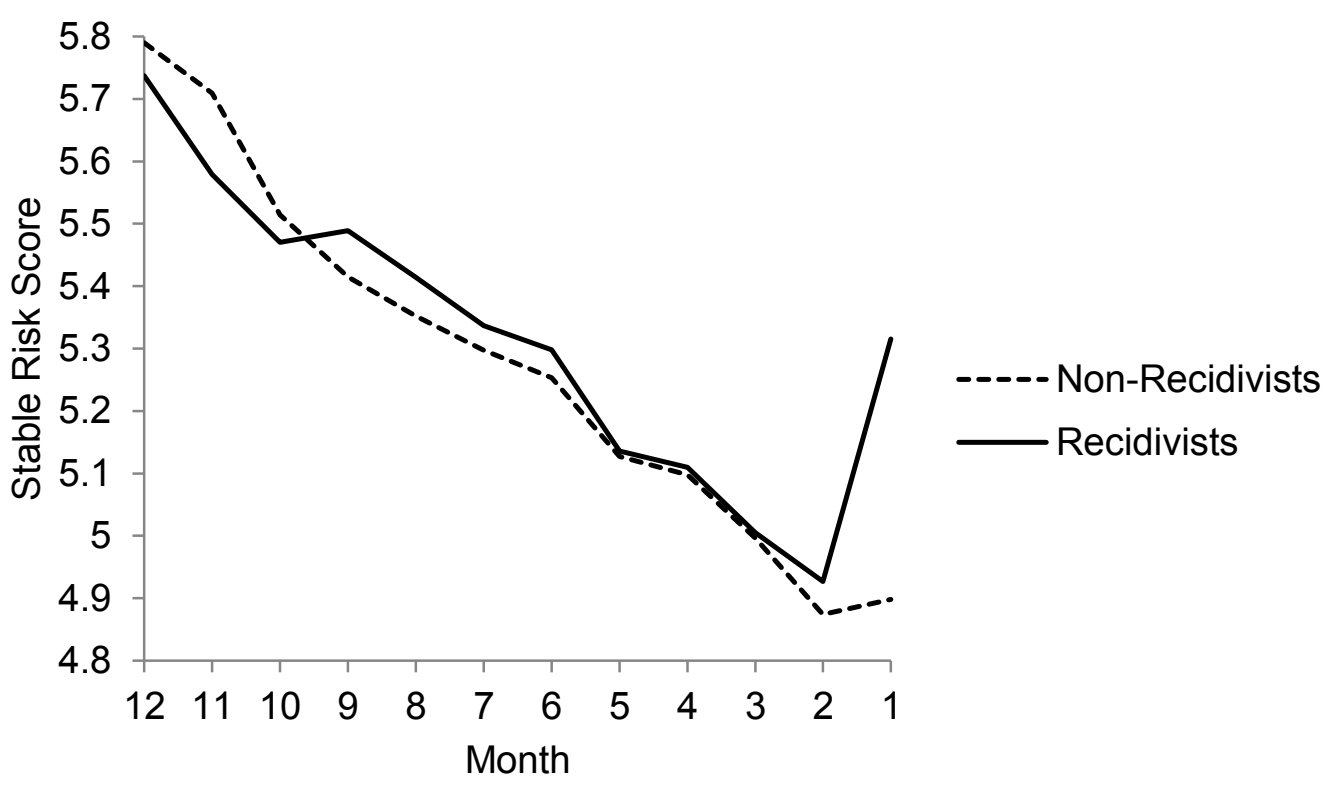

The final model in Table 31 replaces the reconvictions predictor at the intercept and slopes with criminal reconvictions only. Similar to all reconvictions, the Stable Risk scores of recidivists with criminal reconvictions peak in the month prior to recidivism 
relative to non-recidivists in the month prior to censoring (refer to Figure 15). However, there are larger differences between recidivists and non-recidivists in the 12 months prior to follow-up end, with non-recidivists actually scoring significantly higher than recidivists. Their Stable Risk scores eventually converge over time, with non-significant differences between the two groups in Months 2 and 3.

Figure 15. Graph of Stable Risk Criminal Reconvictions Model using monthly variables.

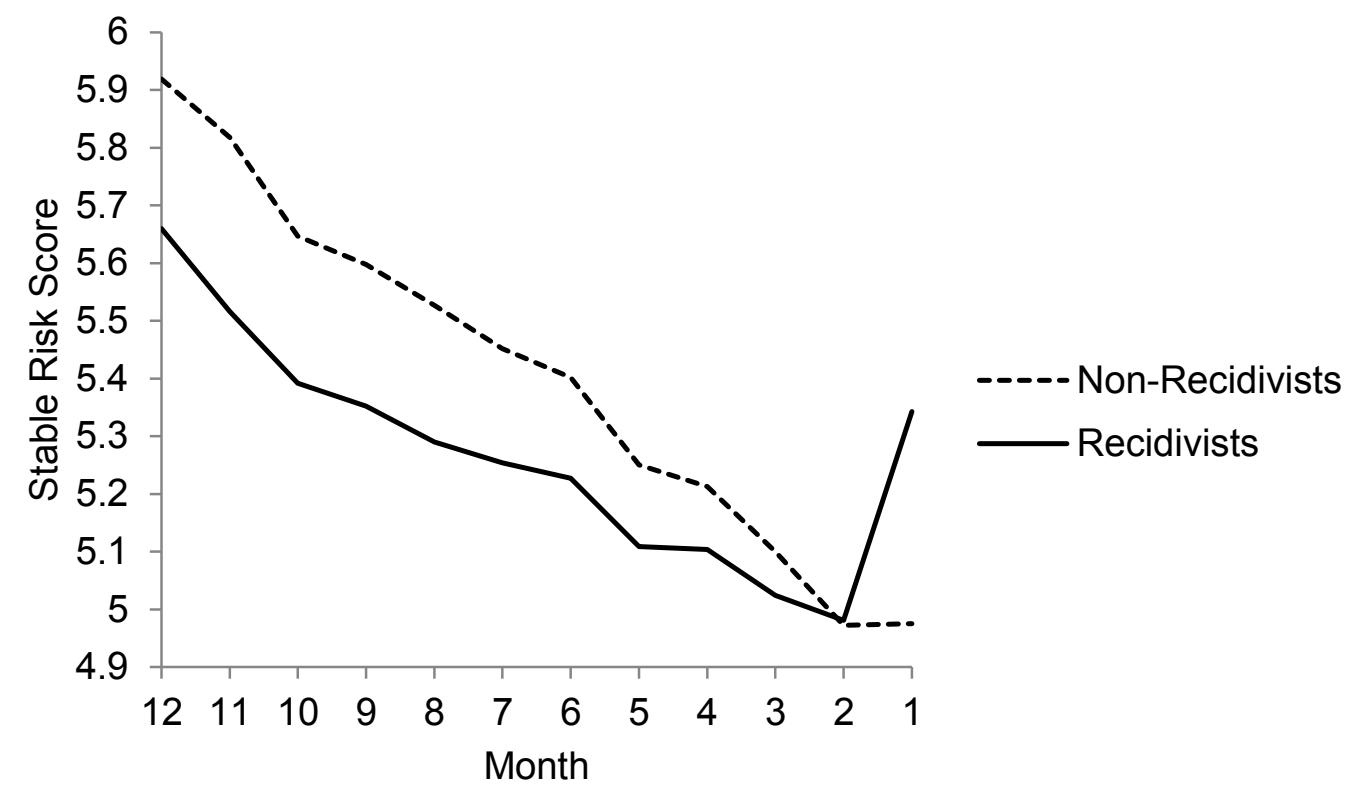

Acute risk. The unconditional growth model for Acute Risk in Table 34 has a grand mean score of 4.48 across all offenders and assessments in the month prior to follow-up end. Figure 16 demonstrates that Acute Risk scores decrease over time in the 12 months leading up to recidivism or censoring, with the exception of the second month prior to follow-up end which shows a slight increase. Differences between recidivists and non-recidivists were examined to explain the slight plateau in scores from Months 2 to 1 . The level-1 residual variance indicates an average scatter of 1.81 of offender's observed outcome values around their own true change trajectory. The level-2 residual 
variance of 4.75 summarizes the between-offender variability in initial status in the baseline month.

Figure 16. Graph of Acute Risk Unconditional Growth Model using monthly variables.

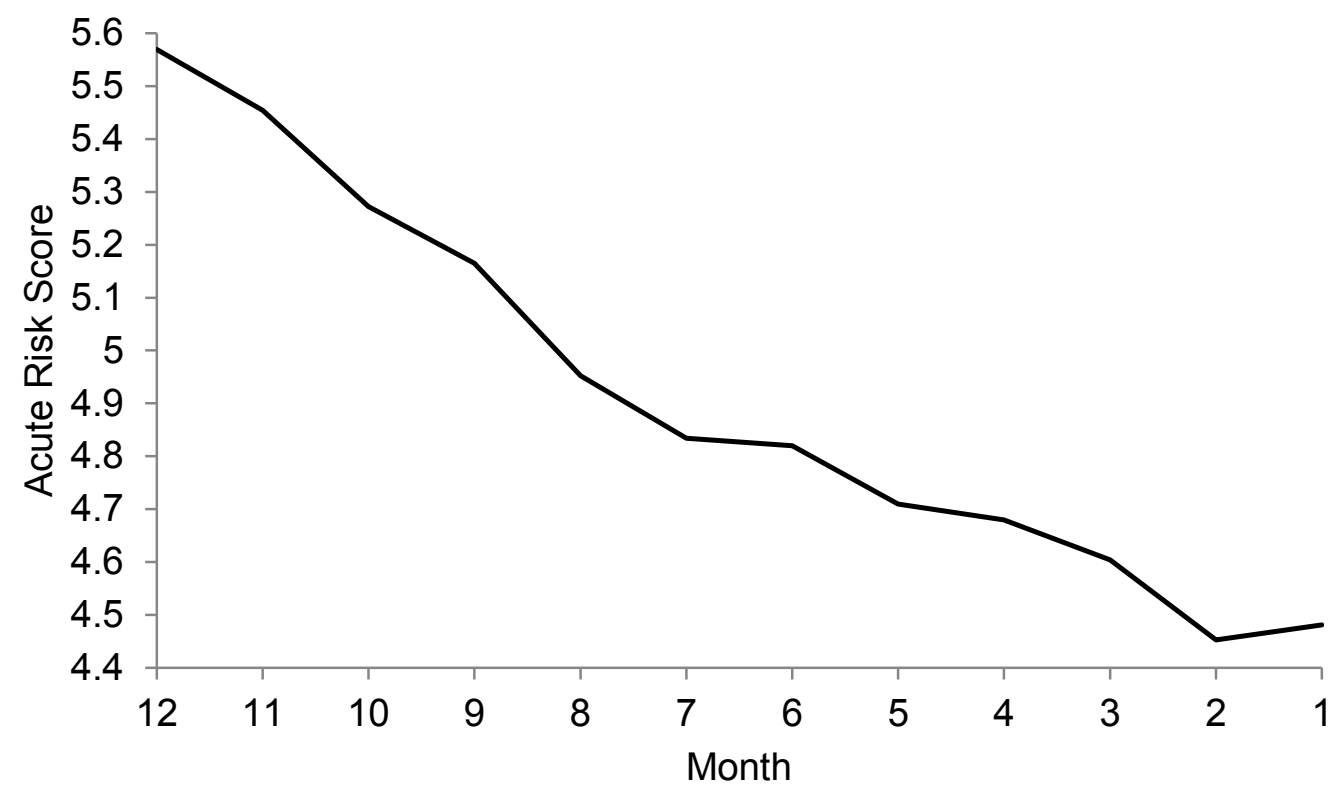

Recidivists and non-recidivists demonstrate a similar rate of change in the 12 months prior to recidivism or censoring. Figure 17 shows a steady decline over time in both groups, with some months where recidivists score higher in Acute Risk and some months where non-recidivists score higher. Recidivists have significantly higher scores than non-recidivists in the ninth and second month prior follow-up end. However in the month prior to recidivism, recidivists do not differ significantly from non-recidivists in the month prior to censoring. 
Figure 17. Graph of Acute Risk All Reconvictions Model using monthly variables.

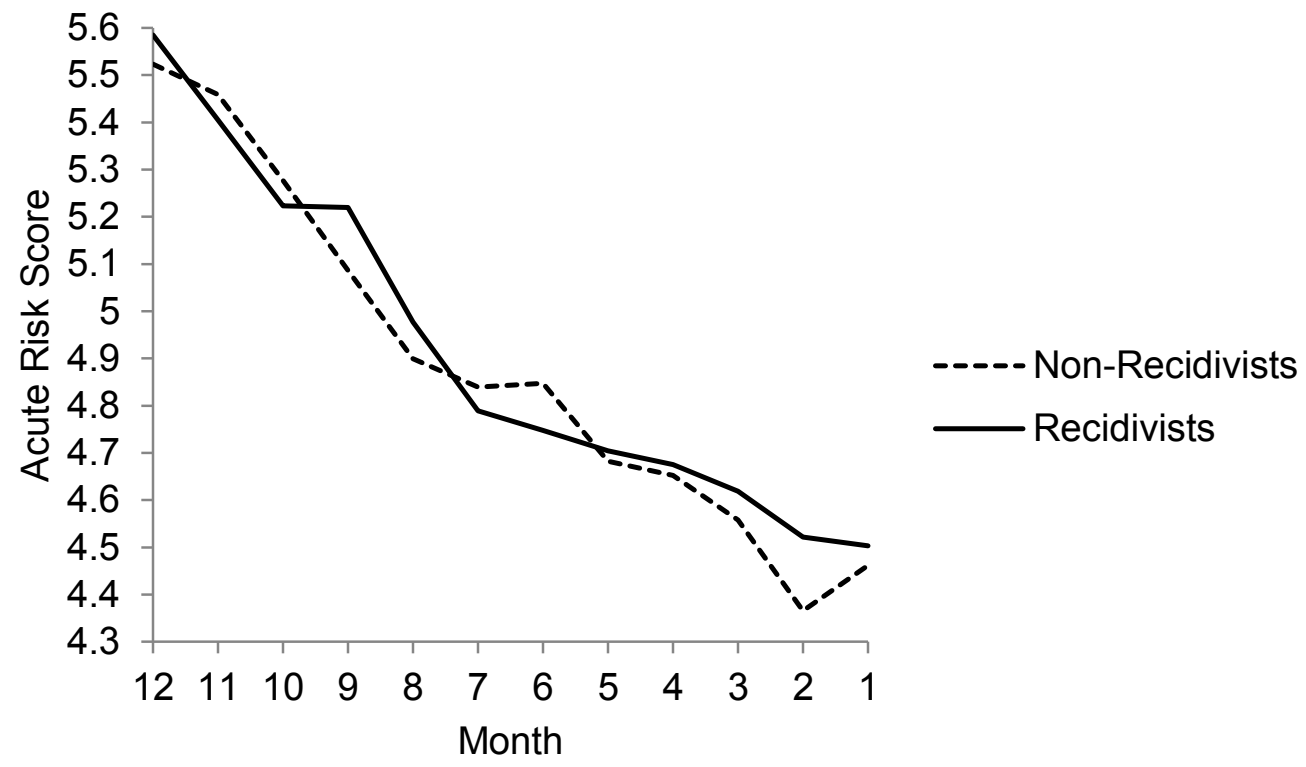

Figure 18 shows a similar pattern of change between recidivists with criminal reconvictions and non-recidivists. Recidivists score significantly higher than nonrecidivists in Acute Risk in Month 2, while non-recidivists score higher in Months 6 and 11. The groups do not differ in the month prior to recidivism or censoring, nor at the beginning of the 12 months.

Figure 18. Graph of Acute Risk Criminal Reconvictions Model using monthly variables.

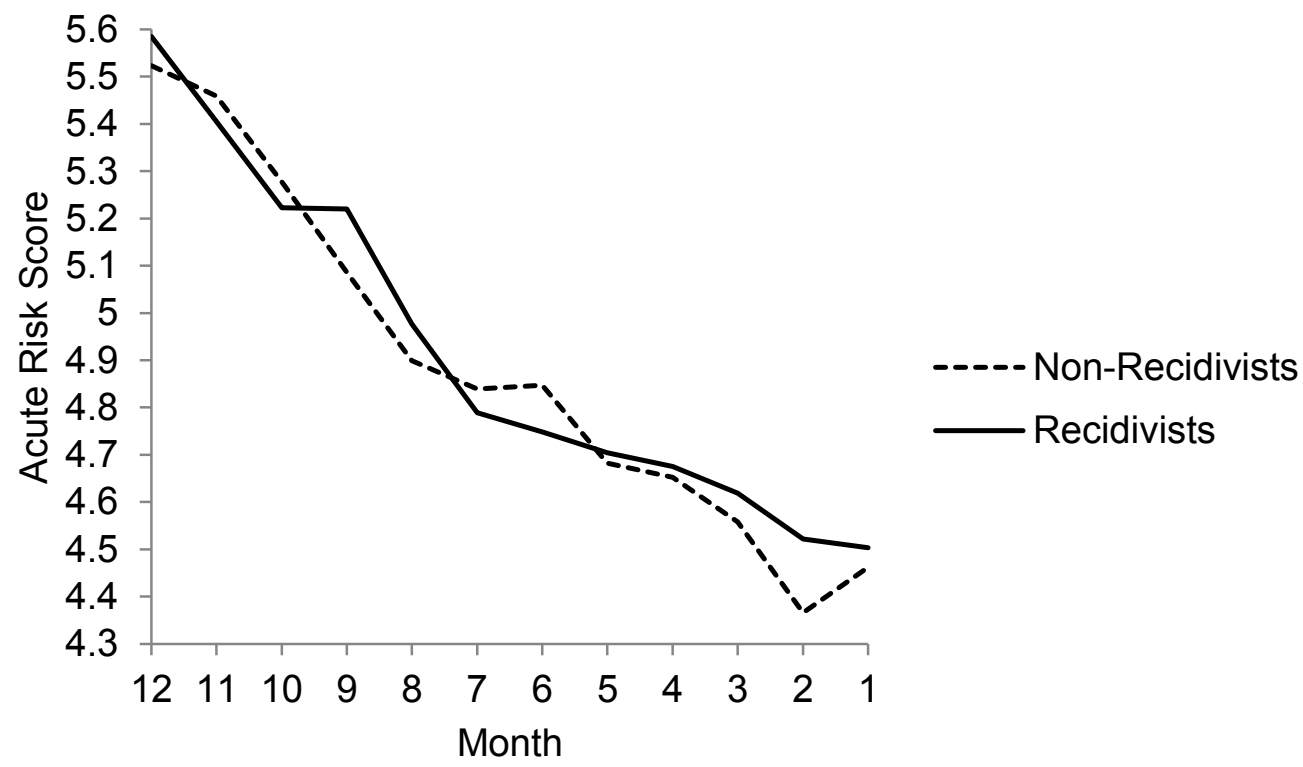


Protective factors. The unconditional growth model displayed in Table 35 provides an estimated average score of 7.50 in Protective Factor scores in the month prior to follow-up end. The between-offender variability in initial status is 6.67. Each offender's data has a scatter of 1.41 around his or her own change trajectory in Protective Factor scores. Unlike Stable Risk and Acute Risk, Protective Factors increase significantly each month over the 12 months prior to recidivism or censoring (see Figure 19). There are no apparent peaks or plateaus when examining all offenders.

Figure 19. Graph of Protective Factors Unconditional Growth Model using monthly variables.

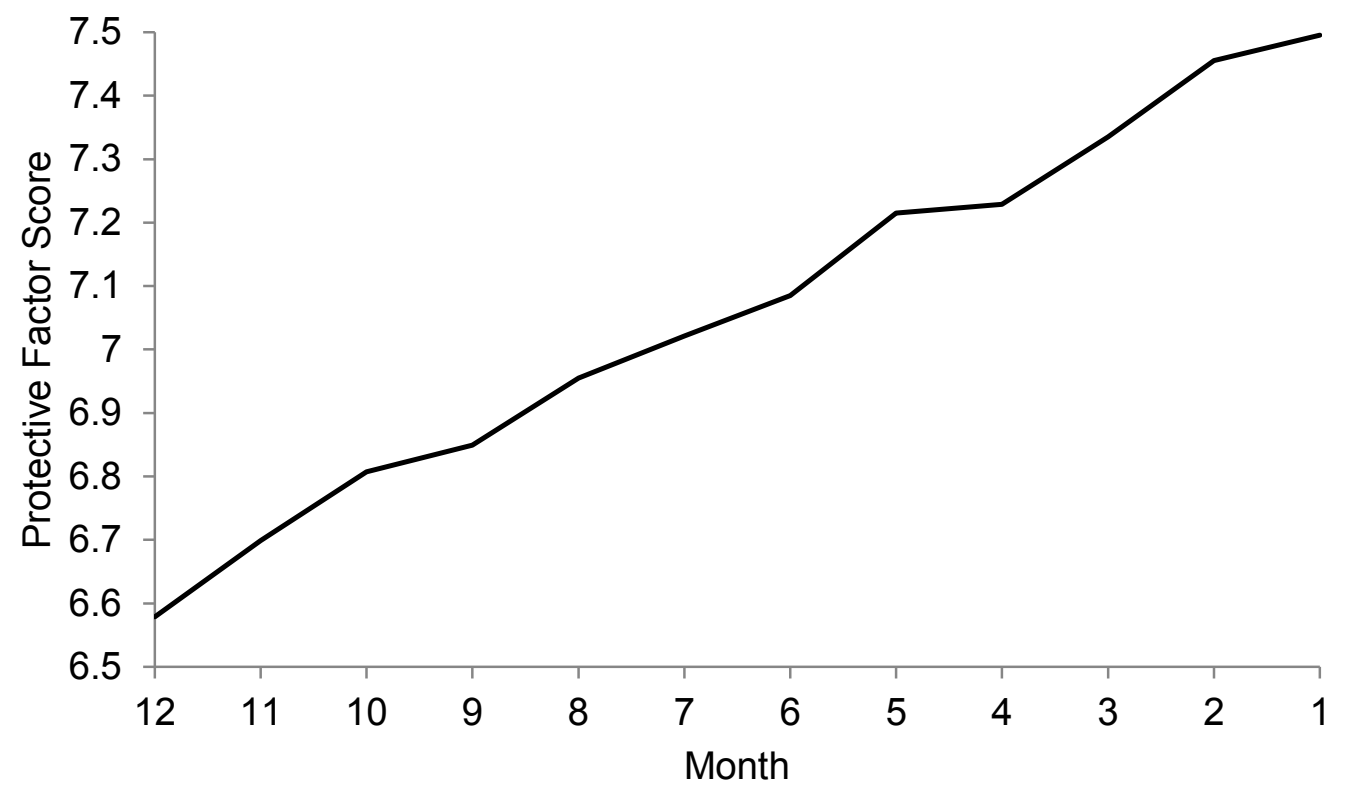

When examining all reconvictions in Figure 20, it appears that recidivists generally score higher than non-recidivists in Protective Factors in the 12 months prior to reconviction. Their scores are significantly higher at Months 5 and 10. In the third month prior to follow-up end, recidivists begin to demonstrate lower Protective Factor 
scores than non-recidivists. This difference reaches significance in the month prior to recidivism for recidivists or censoring for non-recidivists.

Figure 20. Graph of Protective Factors All Reconvictions Model using monthly variables.

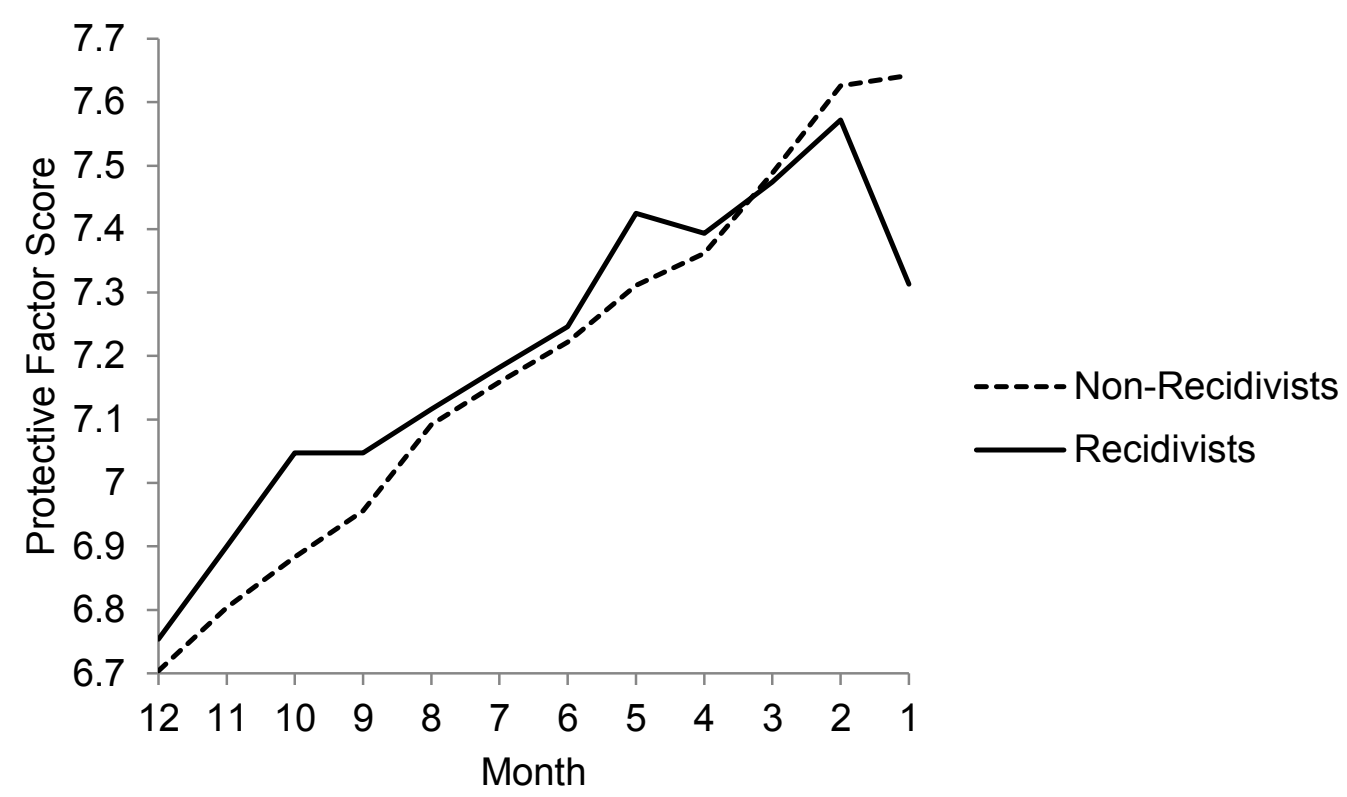

The differences between recidivists and non-recidivists are even more apparent using criminal reconvictions to categorize groups. Figure 21 demonstrates that from Month 12 to Month 4, recidivists score significantly higher than non-recidivists in Protective Factors. Their scores converge in the second and third months prior to recidivism or censoring. Then in the final month prior to follow-up end, the Protective Factor scores of recidivists suddenly dropped to a level significantly lower than nonrecidivists. 
Figure 21. Graph of Protective Factors Criminal Reconvictions Model using monthly variables.

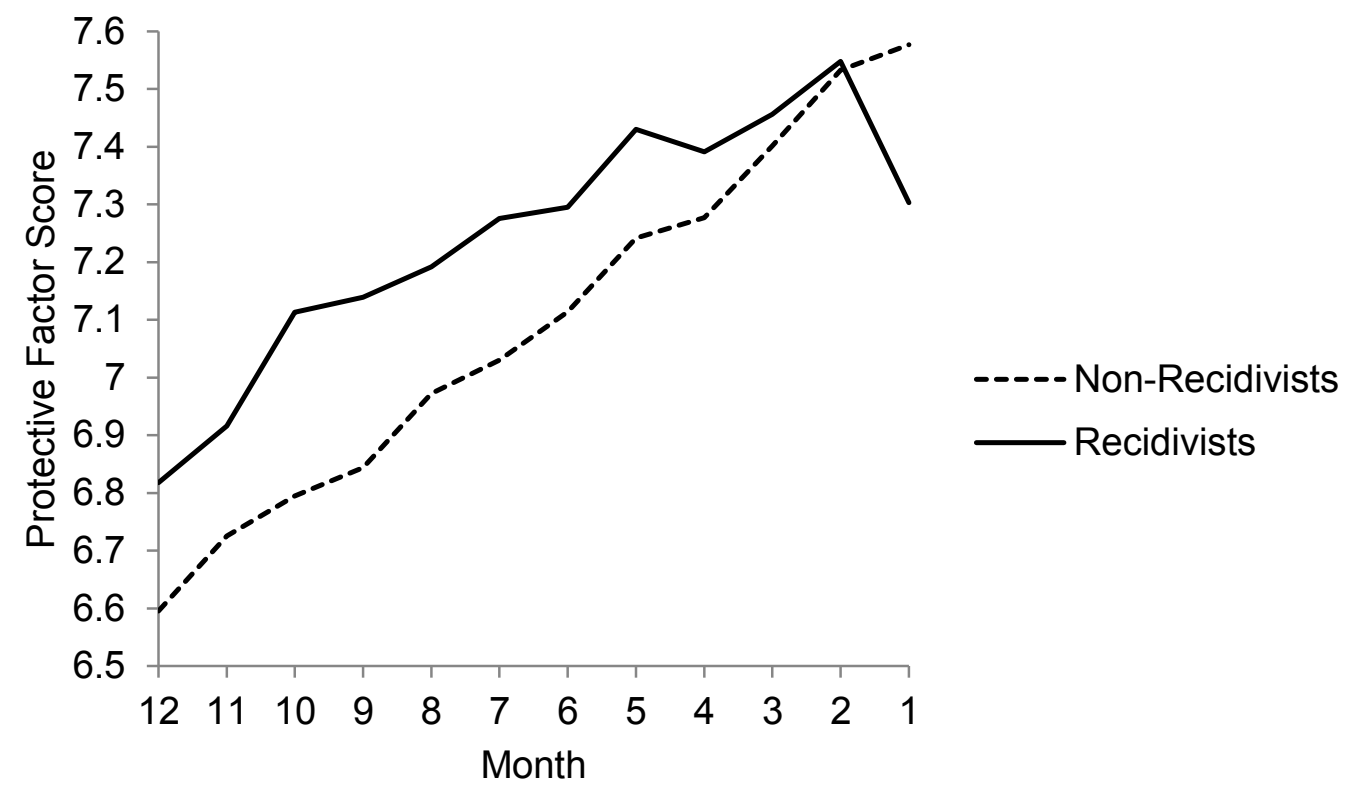

Cox regression survival analysis. Cox regression survival analysis was used to examine whether variation in the risk of recidivism occurrence varies systematically with dynamic risk and protective factor covariates. The event under study was the discrete occurrence of recidivism, first using all reconvictions, then criminal reconvictions only. The time variable used in the analysis was the number of days between parole start date and the follow-up end date of July 18, 2012. Essentially, this controls for the follow-up length for each offender. Covariates consisted of monthly averages of Stable Risk, Acute Risk, and Protective Factors, beginning with the month following parole start date. Covariates changed for each of the 12 models representing the monthly averages for 12 months, while the time variable and event variable remained the same from model to model. Censored cases are data that occur before or after the period of observation. In this case, data that occurred prior to parole start date or after study end date are censored. 
All reconvictions. Table 36 shows regression coefficients, degrees of freedom, $p$ values, and odds ratios for each covariate in each of the 12 models. The sample size available for analyses was $N=3481$, therefore the sample size displayed for each model indicates how many cases were excluded from each analysis (e.g., due to no assessments during the given month, or due to recidivism). In each of the models, monthly average scores of Stable Risk were significantly related to survival from recidivism, while Acute Risk was not. Monthly average Protective Factor scores were significantly related to survival from recidivism in the first four months following parole start date. Positive regression coefficients are associated with shorter survival time, while negative coefficients are associated with longer survival. In all of the models, Stable Risk provided the greatest contribution. The odds ratio (i.e., hazard ratio) indicates how the covariates change the odds of surviving. For all models, the odds of surviving are reduced with an increase in Stable Risk scores. For instance in Month 1, the odds ratio of 1.11 for Stable Risk indicates that the hazard of recidivism increases by $11 \%$ for each unit increase in Stable Risk score. ${ }^{17}$ The models for Months 1 to 4 show that the odds of surviving are raised with an increase in Protective Factor scores. For example, the hazard ratio of .91 for Protective Factors indicates that the hazard of recidivism decreases by $9 \%$ for each unit increase in Protective Factor score.

${ }^{17}$ This is calculated by $100 \%$ - $(100 \% *$ Hazard ratio $)$. For example, $100 \%-(100 \% * 1.11)$. 
Table 36

Cox Regression Analysis of DRAOR Variables on Survival Time of Offenders for All Reconvictions

\begin{tabular}{|c|c|c|c|c|c|c|}
\hline Month & $N$ & Covariate & B & $d f$ & $p$ & $\begin{array}{l}\text { Odds } \\
\text { Ratio } \\
\end{array}$ \\
\hline \multirow[t]{3}{*}{1} & 3394 & Stable Risk & .10 & 1 & $<.001$ & 1.11 \\
\hline & & Acute Risk & -.03 & 1 & .05 & .97 \\
\hline & & Protective Factors & -.09 & 1 & $<.001$ & .91 \\
\hline \multirow[t]{3}{*}{2} & 2958 & Stable Risk & .09 & 1 & $<.001$ & 1.09 \\
\hline & & Acute Risk & -.01 & 1 & .57 & .99 \\
\hline & & Protective Factors & -.09 & 1 & $<.001$ & .92 \\
\hline \multirow[t]{3}{*}{3} & 2610 & Stable Risk & .09 & 1 & $<.001$ & 1.09 \\
\hline & & Acute Risk & -.01 & 1 & .74 & .99 \\
\hline & & Protective Factors & -.07 & 1 & $<.001$ & .93 \\
\hline \multirow[t]{3}{*}{4} & 2357 & Stable Risk & .08 & 1 & $<.001$ & 1.09 \\
\hline & & Acute Risk & .02 & 1 & .30 & 1.02 \\
\hline & & Protective Factors & -.6 & 1 & $<.01$ & .95 \\
\hline \multirow[t]{3}{*}{5} & 2038 & Stable Risk & .10 & 1 & $<.001$ & 1.10 \\
\hline & & Acute Risk & .02 & 1 & .34 & 1.02 \\
\hline & & Protective Factors & -.03 & 1 & .15 & .94 \\
\hline \multirow[t]{3}{*}{6} & 1843 & Stable Risk & .11 & 1 & $<.001$ & 1.12 \\
\hline & & Acute Risk & .02 & 1 & .43 & 1.02 \\
\hline & & Protective Factors & -.02 & 1 & .50 & .98 \\
\hline \multirow[t]{3}{*}{7} & 1447 & Stable Risk & .12 & 1 & $<.001$ & 1.12 \\
\hline & & Acute Risk & .02 & 1 & .50 & 1.02 \\
\hline & & Protective Factors & -.04 & 1 & .22 & .96 \\
\hline \multirow[t]{3}{*}{8} & 1245 & Stable Risk & .13 & 1 & $<.001$ & 1.14 \\
\hline & & Acute Risk & .03 & 1 & .39 & 1.03 \\
\hline & & Protective Factors & -.003 & 1 & .92 & 1.00 \\
\hline \multirow[t]{3}{*}{9} & 1104 & Stable Risk & .12 & 1 & $<.001$ & 1.13 \\
\hline & & Acute Risk & .03 & 1 & .39 & 1.03 \\
\hline & & Protective Factors & .01 & 1 & .001 & 1.01 \\
\hline \multirow[t]{3}{*}{10} & 973 & Stable Risk & .12 & 1 & $<.01$ & 1.12 \\
\hline & & Acute Risk & .06 & 1 & .14 & 1.07 \\
\hline & & Protective Factors & .003 & 1 & .94 & 1.00 \\
\hline \multirow[t]{3}{*}{11} & 827 & Stable Risk & .14 & 1 & $<.01$ & 1.15 \\
\hline & & Acute Risk & .03 & 1 & .51 & 1.03 \\
\hline & & Protective Factors & -.02 & 1 & .77 & .99 \\
\hline \multirow[t]{3}{*}{12} & 736 & Stable Risk & .16 & 1 & $<.01$ & 1.17 \\
\hline & & Acute Risk & .06 & 1 & .32 & 1.06 \\
\hline & & Protective Factors & -.03 & 1 & .56 & .97 \\
\hline
\end{tabular}


A comparison of effect sizes for each model indicates whether there is a trend for later assessments to demonstrate higher predictive validity than earlier assessments. The effect size indicates the relative association between survival and the monthly scores tested. Contrary to the hypothesis, assessments taken more proximally to the end of the follow-up period have lower predictive validity for all reconvictions compared to assessments at the beginning of the follow-up period. As can be seen in Figure 22, the effect sizes for all reconvictions are highest in the month following parole start date and show a decline until Month 12 in which the effect size increases.

Figure 22. Effects sizes for 12 models based on monthly average DRAOR subscales.

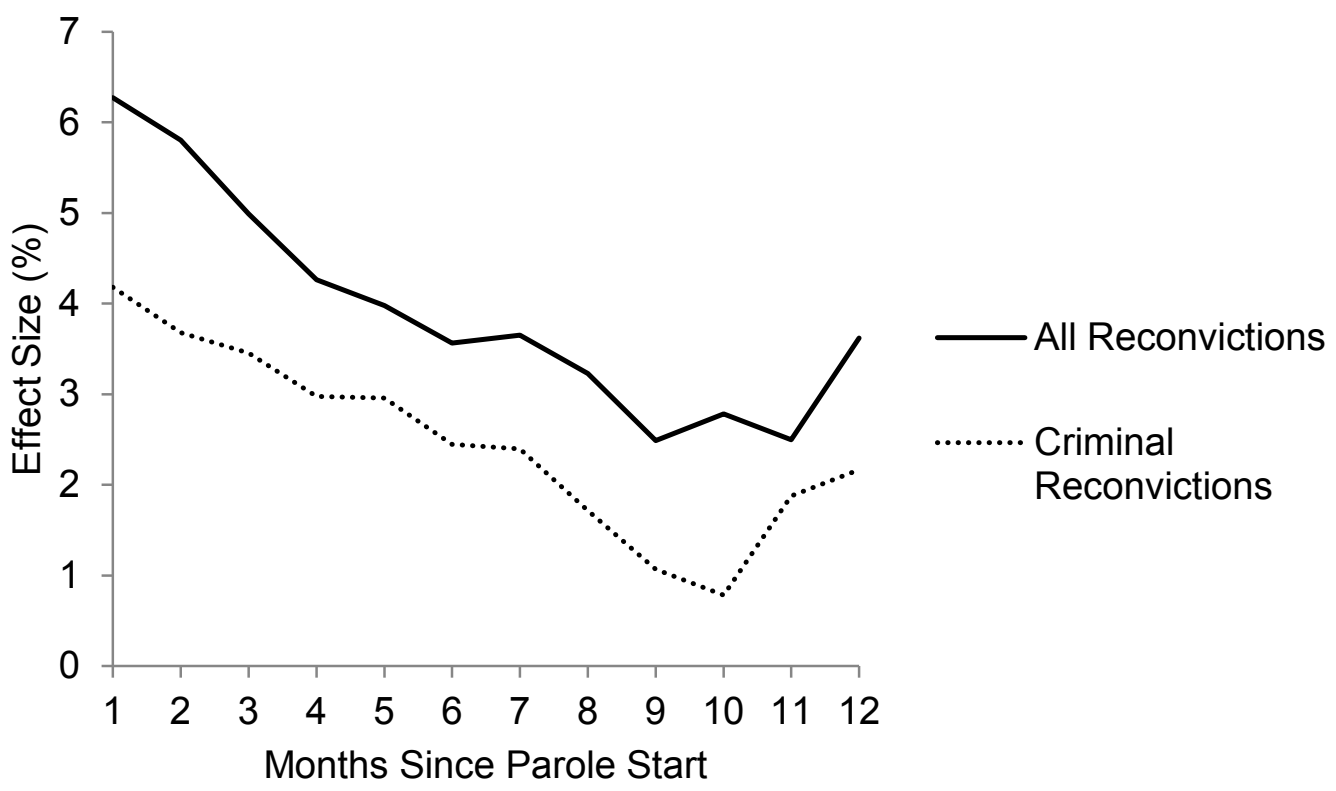

Criminal parole failures. The preceding analysis was repeated using criminal reconvictions to dichotomize recidivists and non-recidivists. As displayed in Figure 22, the relative association between dynamic risk and protective factor covariates and failure/survival is less pronounced when examining criminal reconvictions only. Effect 
sizes decreased in the first 10 months following parole start date, and begin to increase in Month 11. Findings for the 12 models are displayed in Table 37. Similar to all reconvictions, monthly average Protective Factor scores were significantly related to survival from criminal reconvictions only in the first four months following parole start. The greatest contribution was provided by Stable Risk; monthly average scores were significantly related to survival using all 12 months except for Month 10 (which approached significance). Acute Risk scores were not related to survival time. Therefore, the odds of surviving are reduced with an increase in Stable Risk score in all months (with the exception of Month 10), while they are raised with an increase in Protective Factor score in the first four months only.

Summary: Part 6. Differences were observed between recidivists and nonrecidivists in many of the 12 months prior to recidivism or censoring for each of the subscales. A sharp increase in Stable Risk and decrease in Protective Factors occurred in the month prior to recidivism for recidivists. However, support was not found for Hypothesis 7 as the highest predictive accuracy was observed in monthly average scores in the month after parole start, with lower predictive accuracy with more proximal assessments. 
Table 37

Cox Regression Analysis of DRAOR Variables on Survival Time of Offenders for Criminal Reconvictions

\begin{tabular}{|c|c|c|c|c|c|c|}
\hline Month & $N$ & Covariate & B & $d f$ & $p$ & $\begin{array}{l}\text { Odds } \\
\text { Ratio }\end{array}$ \\
\hline \multirow[t]{3}{*}{1} & 3366 & Stable Risk & .08 & 1 & $<.001$ & 1.09 \\
\hline & & Acute Risk & -.003 & 1 & .85 & 1.00 \\
\hline & & Protective Factors & -.09 & 1 & $<.001$ & .92 \\
\hline \multirow[t]{3}{*}{2} & 2947 & Stable Risk & .08 & 1 & $<.001$ & 1.08 \\
\hline & & Acute Risk & .004 & 1 & .86 & 1.00 \\
\hline & & Protective Factors & -.08 & 1 & $<.001$ & .93 \\
\hline \multirow[t]{3}{*}{3} & 2596 & Stable Risk & .09 & 1 & $<.001$ & 1.09 \\
\hline & & Acute Risk & .004 & 1 & .83 & 1.00 \\
\hline & & Protective Factors & -.06 & 1 & $<.01$ & .94 \\
\hline \multirow[t]{3}{*}{4} & 2357 & Stable Risk & .08 & 1 & $<.001$ & 1.09 \\
\hline & & Acute Risk & .01 & 1 & .60 & 1.01 \\
\hline & & Protective Factors & -.06 & 1 & $<.05$ & .95 \\
\hline \multirow[t]{3}{*}{5} & 2023 & Stable Risk & .09 & 1 & $<.001$ & 1.09 \\
\hline & & Acute Risk & .03 & 1 & .30 & 1.03 \\
\hline & & Protective Factors & -.04 & 1 & .14 & .96 \\
\hline \multirow[t]{3}{*}{6} & 1843 & Stable Risk & .11 & 1 & $<.001$ & 1.11 \\
\hline & & Acute Risk & .03 & 1 & .34 & 1.03 \\
\hline & & Protective Factors & -.004 & 1 & .89 & 1.00 \\
\hline \multirow[t]{3}{*}{7} & 1447 & Stable Risk & .13 & 1 & $<.001$ & 1.14 \\
\hline & & Acute Risk & .03 & 1 & .46 & 1.03 \\
\hline & & Protective Factors & -.003 & 1 & .94 & 1.00 \\
\hline \multirow[t]{3}{*}{8} & 1245 & Stable Risk & .14 & 1 & $<.001$ & 1.15 \\
\hline & & Acute Risk & .01 & 1 & .85 & 1.01 \\
\hline & & Protective Factors & .02 & 1 & .58 & 1.02 \\
\hline \multirow[t]{3}{*}{9} & 1104 & Stable Risk & .11 & 1 & $<.01$ & 1.12 \\
\hline & & Acute Risk & -.01 & 1 & .80 & .99 \\
\hline & & Protective Factors & -.001 & 1 & .99 & 1.00 \\
\hline \multirow[t]{3}{*}{10} & 973 & Stable Risk & .08 & 1 & .09 & 1.09 \\
\hline & & Acute Risk & .03 & 1 & .58 & 1.03 \\
\hline & & Protective Factors & -.002 & 1 & .97 & 1.00 \\
\hline \multirow[t]{3}{*}{11} & 803 & Stable Risk & .15 & 1 & $<.01$ & 1.16 \\
\hline & & Acute Risk & .06 & 1 & .30 & 1.06 \\
\hline & & Protective Factors & .04 & 1 & .50 & 1.04 \\
\hline \multirow[t]{3}{*}{12} & 730 & Stable Risk & .18 & 1 & $<.01$ & 1.20 \\
\hline & & Acute Risk & .06 & 1 & .46 & 1.05 \\
\hline & & Protective Factors & 03 & 1 & .71 & 1.03 \\
\hline
\end{tabular}




\section{Discussion}

\section{Summary of Findings}

This is the first examination of the psychometric properties of the original DRAOR (i.e., Cronbach's alpha, item-total correlations, correlations with static risk). This study began by examining the construct validity of the DRAOR at the initial, middle, and last assessment periods. The results of an Exploratory Factor Analysis at the initial assessment generated two potential models in addition to the original subscales, depending on the extraction method used. The stability of these models was tested using Confirmatory Factor Analysis and while one model (Model \#3) demonstrated a better fit using the middle DRAOR assessment, the other (Model \#2) best fit the data using the last assessment. However, all three models demonstrated good fit to the observed data and acceptable psychometric properties across time periods. As a comparison, the more theoretically appealing model (Model \#3) was also tested in the between-offender and ROC analyses. The findings did not differ drastically between the original DRAOR Stable Risk subscale and Acute Risk subscale and the model's mixed Stable and Acute factor. In fact, ROC analysis showed that the original Acute Risk subscale had the highest predictive accuracy of recidivism $(\mathrm{AUC}=.72$ for all reconviction, $\mathrm{AUC}=.67$ for criminal reconviction).

In the interest of practical utility and given the acceptable psychometric properties demonstrated by the original DRAOR subscales, it is not recommended that the DRAOR be reduced or subscales revised at this stage. However, further investigation is required at the item-level. In particular, the predictive accuracy and utility of the 'Employment' and 'Interpersonal Relationships' Acute risk items which did not load onto any factor 
require review. At this stage, the operational relevance of these items for probation supervision warrants their inclusion in the scale. Given that the factor structure of the scale was not entirely consistent with the constructs the DRAOR purports to measure (i.e., stable risk, acute risk, and protective factors), construct validity is not fully supported.

Between-offender analyses highlighted the ability of the DRAOR and its subscales to differentiate between recidivists and non-recidivists. As hypothesized, recidivists had higher levels of Stable Risk, Acute Risk, and overall DRAOR scores, and lower levels of Protective Factors than non-recidivists at both the initial and last assessment periods. The highest effect sizes for these analyses were observed for all offenders using the reconviction criterion to distinguish recidivists from non-recidivists. However, significant findings also emerged for all offenders using criminal reconvictions to categorize offenders, as well as for Maori offenders using both the reconviction and criminal reconviction criteria.

The predictive accuracy of the DRAOR was established using ROC analysis. The DRAOR and its subscales consistently predicted recidivism, with higher effect sizes observed for reconvictions over criminal reconvictions. This established the first part of the theoretical litmus test of dynamic variables requiring evidence that DRAOR scores are related to recidivism. Contrary to the hypothesis that recidivism and desistance from crime would be best predicted from Protective Factors, Acute Risk demonstrated the highest predictive accuracy. Higher levels of predictive accuracy were found with all offenders than Maori offenders alone. As hypothesized, the DRAOR predicted all reconvictions and criminal reconvictions above and beyond the RoC*RoI. The combined 
dynamic and static model predicted all reconvictions more accurately than the $\mathrm{RoC}^{*} \mathrm{RoI}$ alone, but not criminal reconvictions. These findings were consistent for Maori offenders.

The second part of the empirical litmus test to support that the DRAOR is in fact dynamic is evidence that scores change over time. Multilevel growth modelling served this purpose with the development of unconditional growth models for Stable Risk, Acute Risk, and Protective Factors, which established that the dynamic risk variables decreased over time while Protective Factors increased. This provided support for the hypothesis that dynamic factors change over a period of systematic assessment and reassessment within an individual offender, and that the DRAOR is sensitive to change. In addition to this evidence of intra-individual change, multilevel growth modelling explored some of the person-level variables that may explain some of this variability in growth. The majority of the variance in dynamic risk and protective factors was observed between offenders, rather than within the offender. For each outcome, the RoC*RoI was found to be a significant person-level predictor of initial scores at first assessment, and rate of change over time for Acute Risk only. Age was found to be a significant control variable for RoC*RoI on Stable Risk and Acute Risk scores at first assessment. Other personlevel variables such as Maori ethnicity, gender, and number of DRAOR assessments did not contribute as much to explaining variance as the models presented.

An additional set of multilevel growth models tested the hypothesis that there would be differences between recidivists and non-recidivists in their initial dynamic risk and protective factor scores and their rate of change over time. Recidivists showed higher levels of Stable Risk and Acute Risk and lower levels of Protective Factors at first 
assessment. Their rates of change did not differ, except in the case of Acute Risk in which recidivists decreased at a slower rate than non-recidivists. This was true for the categorization into recidivists and non-recidivists based on both reconvictions and criminal reconvictions only.

A final set of multilevel models were developed to provide a descriptive explanation of the utility of the DRAOR at different time points. This analysis showed that Stable Risk and Acute Risk scores decreased while Protective Factor scores increased overall in the 12 months leading up to recidivism or censoring. A comparison of recidivists and non-recidivists showed differences in their peaks and plateaus of these dynamic variables. Recidivists and non-recidivists differed in their average Stable Risk and Protective Factor scores in the month prior to recidivism or censoring using both reconvictions and criminal reconvictions to categorize recidivist groups. Although there were no significant differences between groups in the month prior to follow-up end for Acute Risk, higher scores were observed in recidivists in the second month prior to follow-up end.

Lastly, Cox regression survival analysis was used to answer at what point the DRAOR becomes predictive of recidivism. The findings of this analysis indicate that monthly average Stable Risk scores are predictive of reconvictions and criminal reconvictions immediately following parole start and continue to do so for the 12 months following. Monthly average Protective Factor scores predicted both outcomes only for the first 4 months following parole start, while monthly average Acute Risk scores were not predictive in any of the 12 months following parole start. Contrary to the hypothesis that later assessments would demonstrate higher predictive accuracy than earlier 
assessments, the predictive accuracy of the DRAOR declined over the 12 months following parole start.

\section{Implications for Practice}

Empirical litmus test. The empirical litmus test for designation as a truly dynamic factor requires significant group level change over time and a significant contribution to the prediction of recidivism (Brown et al., 2009). The current study provided evidence that the DRAOR meets both of these criteria. Whether examining the number of days since first assessment or monthly scores leading up to follow-up end date, the DRAOR subscales showed significant change across time. Although there were some differences depending on the timing of assessment, the DRAOR predicted recidivism both in the form of reconvictions and criminal reconvictions. As such, the DRAOR, as a dynamic risk scale, can potentially inform case planning and improve casebased decisions.

Jones (1996) argued that inherent fluctuations in dynamic variables make them unstable, which limits their reliability and validity. Differences were observed in dynamic variables between recidivists and non-recidivists in the 12 months prior to recidivism or censoring. Instead of viewing this as a drawback, the foundation of this study is that continually re-assessing dynamic risk using the DRAOR takes into account the offender's current criminogenic state and changes in the environment. The utility of this approach allows for appropriate changes to supervision strategies to be implemented depending on changes in dynamic risk and protective factors. Not only do dynamic risk factors tell us when an offender is at a higher risk to reoffend, but also how to reduce that risk (Hanson, 2006). An unexpected finding of this study was the declining predictive 
accuracy of the DRAOR over time. However, the last assessment better differentiated recidivists from non-recidivists compared to the initial baseline assessment. Previous research indicates that offenders who fail tend to do so in the first three to six months after release (Brown et al., 200; Solomon et al., 2005). A possible explanation for the decrease in predictive validity is that the DRAOR may be more predictive for higher risk offenders who tend to reoffend earlier after release.

While recidivists and non-recidivists did not differ in dynamic risk and protective factors in every month prior to follow-up end, they did exhibit significant differences in Stable Risk and Protective Factors in the month prior to recidivism or censoring. While it is unlikely that a single baseline assessment is adequate to accurately capture requisite risk factors and strengths, reassessment of dynamic factors (both risk and protective) may improve prediction of when an offender has a greater probability of reoffending.

Differences were observed between recidivists and non-recidivists in average Acute Risk scores in the second month prior to follow-up end, but not in the month prior. However, Acute Risk showed the highest levels of predictive accuracy in the last assessment before recidivism or censoring. These seemingly contradictory findings highlight the complexity of these dynamic factors and provide some insight into the distinction between stable and acute dynamic risk factors. Hanson (2006) suggested that the last assessment before recidivism provides the most information when examining characteristics of short duration and when risk is only increased when the factor is present at the time of the offence. However, Hanson and colleagues (2007) found that the average acute risk assessment over a six-month period was a better predictor of recidivism than the most recent assessment. The findings of this study are in line with 
the conceptualization of acute risk as changeable within days, hours, or even minutes (Hanson \& Harris, 2000). The effect of a spike in Acute Risk factors seems to be very narrow in that only one assessment or a few assessments is indicative of imminent recidivism. In this case, parole officers need to be extra vigilant if offenders show higher than normal Acute Risk scores during one contact. Consistent with Hanson (2006), this suggests that knowledge of acute factors is required to determine what areas need to be monitored on supervision.

On the other hand, recidivists and non-recidivists showed significant differences in their Stable Risk factors in the month prior to recidivism or censoring, as well as most other months in the 12 months preceding criminal reconvictions in particular. Knowledge of stable risk factors is required for offenders to know what they need to change and for probation staff to know what should be addressed in treatment (Hanson, 2006), and for a referral to an appropriate program or intervention if necessary. The difference in scores between recidivists and non-recidivists at first assessment but not in their rate of change over time highlights the importance of criminogenic programming targeting these factors, especially earlier in their release or pre-release.

This study provided the refined longitudinal analysis needed to distinguish between these two aspects of dynamic risk. These findings are consistent with the existence of a genuine distinction between stable risk as durable and acute risk as transient. These different aspects of risk are important at various points of contact in the community (Hudson et al., 2002). As such, the relationship of certain dynamic risk factors to outcome may be moderated by the timing of the assessment (Jones et al., 2010). The factor structure of these two factors suggests their distinction needs further clarity. 
Reconceptualization of acute risk into internal and external factors would be in line with current desistance work (Serin, Lloyd et al., 2010). While the definition and clarity of these dynamic factors is problematic and there remains confusion over their predictive validity, they remain relevant at a theoretical and empirical level.

The role of protective factors. Consistent with Farrington and Loeber (2000) and Newcomb and Felix-Ortiz (1992), protective factors are conceptualized in this research as distinct factors from dynamic risk. While the factor structure of the dynamic risk items of the DRAOR was inconsistent with the original Stable Risk and Acute Risk subscales, it consistently confirmed Protective items as a distinct factor. The SAPROF (De Vogel et al., 2007), for example, considers living circumstances, work, and intimate relationships as protective variables. The DRAOR classifies these variables as acute dynamic risk items and the findings of factor analyses did not suggest these variables clustered with protective items. Instead they should remain grouped with other aggravating variables, while variables that mitigate risk should remain distinct but can co-exist with dynamic risk variables.

The findings of this study provide support for protective factors as being contextspecific and dynamic in nature. After parole start, both recidivists and non-recidivists gain more internal assets and social support over time. In the 12 months prior to followup end, recidivists actually have higher Protective Factor scores in some months.

However, in the month prior to recidivism or censoring, we see a sharp drop in the scores of recidivists, becoming significantly lower than other offenders. This pattern is even more striking when criminal reconvictions in particular are examined. Recidivists show greater Protective Factors in the 12 months prior to follow-up end, but their scores begin 
to converge a few months prior with a sudden drop in the month prior to recidivism. The variables that make up the Protective Factor subscale suggest that something is happening in their lives, either internally (e.g., shift in expectations) or externally (e.g., social support breaking down) that removes the protective buffer they previously had. Parole officers may be alerted to imminent recidivism if they notice a loss of insulating strengths over the course of a few weeks, coinciding with a change in lifestyle stressors or disinhibitors (reflected in an increase in Acute Risk scores) during one or possibly a few parole contacts.

One of the conceptual issues raised and tested by Ullrich and Coid (2011) is whether protective factors have stable effects over time or whether they are time dependent. Violent reoffending was the outcome variable in Ullrich and Coid's study, and they found that the effects of some protective factors (i.e., social support, family time with friends/family) were stable across time post-release (mean follow-up time $=5.3$ years). Some protective factors had an influence on reduction of risk shortly after release (i.e., emotional support, place to stay after release) in the community and some were not relevant until the long term (i.e., closeness to others, working/training/education). While the current study did not measure individual protective items, it does suggest that the influence of protective factors is different depending on the timing of assessment. The Protective Factor subscale of the DRAOR represents more internal assets and prosocial identity changes (as well as social support). The protective variables that comprise the DRAOR may be sufficient for explaining why parolees remain crime-free in the first four months after parole start, but insufficient for explaining why parolees continue to refrain from recidivism beyond four months post-release. Given that offenders tend to reoffend 
in the three to six months after release (Brown et al., 2009; Solomon et al., 2005), these findings may suggest that protective factors are more useful for higher risk offenders that reoffend in the first four months after parole start. Since it appears that protective factors play an additive or complementary role to stable risk factors during this time, parole planning needs to enhance protective factors and target stable risk factors as early as possible following release.

Once the relationship between protective factors and recidivism has been further validated and explained, risk management strategies should be developed for individual offenders taking into account their internal and external strengths that may protect them from reoffending. Ullrich and Coid (2011) found that protective factors did not have different effects on different levels of risk. The risk factors weakened protective mechanisms, but the independent effects of protective factors were found whether the offender was low, moderate, high, or very high risk for future violence. Although Fitzpatrick's (1997) mediation and buffering models were not directly tested in the current study, the effects of protective factors on reconvictions were independent of stable and acute dynamic risk only for the first four months following parole start. This is consistent with Ullrich and Coid's findings and Fitzpatrick's mediation model. A lack of independent effects past four months post-release may indicate that offenders are more likely to engage in criminal behaviour when risk factors are high and protective mechanisms are low or absent, consistent with the buffering model. Since these models were not tested directly, further research is needed to determine how individual dynamic risk and protective factors interact over time and should be incorporated into risk assessments. 
Relationship with static risk. As expected, the combined static and dynamic model provided the highest predictive accuracy for all reconvictions using the $\mathrm{RoC} * \mathrm{RoI}$ score at release and the DRAOR assessment prior to recidivism. While the combined static and dynamic model did not demonstrate higher predictive accuracy of criminal convictions than static risk alone, the DRAOR added incremental validity to the RoC*RoI. The DRAOR appears to be more sensitive to technical violations, consistent with its intended goals. The accuracy of this approach is founded in the measurement of the risk status of the offender, or their risk relative to other offenders at release. The DRAOR taps into an offender's propensity to commit an offence at a given time. A more refined differentiation of offenders showing similar levels of static risk may augment case management, determine suitable levels of frequency of contact, and appropriately allocate resources. The findings of this study confirm that risk state changes over time. Such change may be influenced by various factors, including parole/probation officer intervention (Bonta et al., 2008, 2011; Bourgon \& Gutierrez, 2012; Robinson, VanBenschoten, Alexander, \& Lowenkamp, 2011; Robinson et al., 2012).

Static risk factors at release and dynamic factors post-release address different issues instead of competing for outcome variance (Quinsey, Jones et al., 2006). Risk assessment requires an accurate picture of long-term risk and sensitivity to short-term changes in the likelihood of reoffending (Quinsey, Jones et al., 2006). The combined use of the RoC*RoI and the DRAOR fulfills both of these goals. From a theoretical perspective, static factors provide an insufficient scientific explanation in that causal factors should be contiguous with their effects (Hanson, 2006). A static assessment of risk at release ignores everything that occurs in the three months, six months, or one year 
between release and recidivism. The DRAOR provides an incremental advantage over the $\mathrm{RoC}^{*} \mathrm{RoI}$ given that its purpose extends beyond risk assessment to risk management and reduction.

It would be expected that the frequency of contact between parole officer and parolee would be proportional to the risk level of the parolee. Bonta and colleagues (2008) found that the number of contacts was positively related to risk score in adults in the first three months of supervision. This is explained by high-risk offenders being seen more frequently than low or moderate risk offenders. The standard in New Zealand is a minimum requirement for offenders to report at least once every 10 business days or every 40 business days for those offenders on life parole. However, it is up to the parole officer's discretion to increase or decrease the frequency of reporting; changes in the DRAOR could inform such discretion. Overall, there were no differences in the number of DRAOR assessments by static risk group. When breaking this down into 3-month increments following parole start date, it emerged that low-risk offenders had fewer assessments than medium- or high-risk offenders from parole start date to 6 months postrelease.

This is consistent with Andrews and Bonta's (2010a) risk principle in that offenders with higher levels of static and dynamic risk factors require a higher frequency of contact. Static factors may guide the frequency of reporting in the first few months following release, then utilizing the DRAOR can help parole and probation officers guide their frequency of contact as risk level changes. This is important given that oversupervising low-risk offenders can increase their likelihood of recidivism (Lowenkamp \& Latessa, 2004). An offender with increasing dynamic risk factors and decreasing 
protective factors would require a higher level of supervision and intervention. When resources are limited, decisions must be made regarding where they should be targeted. This is best done on the basis of objective information about which offenders are most likely to reoffend, and when they are most likely to offend.

Pathway model of desistance. The purpose of regular contact between the offender and probation officer is to maintain contact with the offender, discuss progress with his or her objectives, and identify any emerging risks. The DRAOR helps provide the structure to carry out these goals and to help focus the probation officers' judgment based on empirically-informed risk and protective factors. Advancing the efficacy of the re-entry movement requires more systematic assessment and less focus on offender deficits (Serin, Lloyd et al., 2010). Few studies have examined rapidly changing risk factors such as mood and situational triggers (Brown, 2002). Although dynamic factors such as the Acute Risk factors that make up the DRAOR are extremely difficult to measure, the systematic reassessment showed evidence of change over time. The current research also highlights the importance of considering more proximal and protective factors in the management of risk of community supervised offenders. A focus solely on factors that increase risk has resulted in a gap in our understanding of factors that may protect against reoffending. While Stable Risk and Acute Risk can be construed as offender deficits, Protective Factors can be considered the strengths of the offender and were found to predict reconvictions and criminal reconvictions. Assessing both dynamic risk factors and protective factors provides a more balanced approach and a more accurate risk assessment. Overall, this approach reflects a move from simply the classification of risk to the management and reduction of risk. 
A crime acquisition model may be insufficient in explaining recidivism. These findings of this study provide support for the insulating effect of protective factors against a re-involvement in criminal behaviour. Assessments that measure only risk factors, whether static or dynamic, may produce biased predictions of reoffending (Rogers, 2000). Human behaviour is multi-determined and thus risk factors may explain why an individual reoffends while protective factors may explain why an individual refrains from reoffending. The DRAOR integrates risks and needs with our current understanding of crime desistance. Desisting from crime is a different process from initially getting involved in crime (Kazemian, 2007), thus it is important to consider not only risk factors for engaging in crime, but also factors that buffer or mediate an offender from reoffending. The findings of this study reinforce a movement in risk assessment to include factors related to desistance. Utilizing the DRAOR in conjunction with the principles of risk, need, and responsivity will enable probation officers to take into consideration the pathways that offenders take in desisting from crime.

The structure of the DRAOR thus encourages probation officers to be effective "agents of change" (Bonta et al., 2008; Bourgon et al., 2011, 2012). This is important given that poor compliance has been observed between assessment and case management or action plans (Bonta et al., 2008; Harris et al., 2004). In light of recent findings regarding the limited impact of community supervision (Green \& Wink, 2010; Solomon et al., 2005), parole officers are encouraged to move beyond case management into the role of agents of change. Change agents are required to translate the results of dynamic risk assessment into a therapeutic plan to facilitate change (Bourgon et al., 2012). This approach may ensure a more consistent follow through between risk assessment and case 
management. The results of this study support the use of stable and acute risk factors as suitable targets for case planning. It also may be possible to motivate offenders by concentrating on modifying their dynamic risk factors (Andrews et al., 2011).

Maori offenders. The last issue that warrants discussion is the validity of the DRAOR in Maori offenders. Similar to Aboriginal people in Canada, Maori are overrepresented in the offender population. At the start of this study, Maori represented 44.1\% of all community sentence types (New Zealand Department of Corrections, 2011). One possible explanation for their disproportionate representation is that Maori people are subject to a range of early-life social and environmental factors resulting in a greater risk of engaging in crime (New Zealand Department of Corrections, 2007). Although the DRAOR captures some of these ongoing dynamic factors (e.g., substance abuse), it may not account for the unique needs of Maori offenders. This could explain why the predictive accuracy of the DRAOR was slightly lower in Maori offenders than with the full offender sample. Effect sizes were smaller when focusing solely on Maori offenders for the RoC*RoI and all DRAOR subscales. However, the measure demonstrated medium effect sizes, which are still within the range of good risk assessment tools. The role of ethnicity was previously explored as a potential predictor in the RoC*RoI, finding that its exclusion did not result in a measurable reduction in predictive accuracy (New Zealand Department of Corrections, 2007). Further research is warranted to determine whether a Maori-specific DRAOR tool can improve its psychometric properties with this population, or whether an ethnicity variable could improve the predictive accuracy of the current DRAOR. Based on the development of the RoC*RoI, it is likely that the 
inclusion of ethnicity as a predictor variable will not drastically increase the predictive power of the DRAOR, but could be worthwhile for public policy reasons.

\section{Limitations of the Research}

The study of risk assessment and risk management as well as dynamic factors in particular has flourished in the past few years. However, a number of methodological, theoretical, and statistical weaknesses of past multi-wave studies of dynamic risk factors have been highlighted by Brown and colleagues (2009). These shortcomings include single- or two-wave designs, small samples, and a failure to include rapidly changing risk factors (e.g., mood and situational triggers). It has been recommended that dynamic risk research should include a breadth of dynamic risk indicators, encompass a minimum of three measurement occasions, and be prospective and multi-wave in nature (Brown et al., 2009; Douglas \& Skeem, 2005; Jones et al., 2010). This longitudinal study of dynamic risk assessment met all of these guidelines, with the exception of a prospective research design. However, an archival design was necessary to allow for a longitudinal study with a sufficient follow-up period. As a positive, many of the limitations that often mark prospective studies such as attrition and missing data were not an issue in the current study.

There were a number of potential confounds in the data, many of which were controlled for in the analyses. As the multilevel growth modelling results indicated, the effects of many of these variables (e.g., length of time in the community, number of assessments per offender, length of time between first and last assessment) did not need to be removed from the analysis. There were some variables that were not captured in the data, and therefore could not be controlled. As with many correctional studies, 
recidivism data is collected in the form of reported crime only, as opposed to other measures such as self-report. As such, the classification of offenders into "recidivists" and "non-recidivists" is based on a conservative measure of recidivism and likely does not capture all offenders who reoffended during the study period. Further, the fixed follow-up period produces the potential for sample censoring. Given the overlapping data collection period and follow-up period, some offenders were followed up for as few as four months. However, sample censoring is not a major concern due to the frequency of DRAOR assessments and the retrospective design of the study. Lastly, a DROAR assessment is completed by the probation officer during each "quality" contact with the offender. The duration of these sessions is unknown. Bonta and colleagues (2008) found that sessions with a sample of probation officers were 22 minutes on average and this length was effective for offender change. Such time may not be required for an accurate risk assessment but if the DRAOR is to be used within case planning and risk management, then resource allocation is an important consideration. The efficacy of the sessions in the current study cannot be evaluated since the duration of the sessions is unknown and other variables relating to its effectiveness were not measured.

The study start date coincided with the implementation of the DRAOR for all offenders released on parole in New Zealand. Although probation officers were supposed to apply the DRAOR with all quality contacts, it is clear from the total number of assessments completed each month that it was used sporadically at first. There was no information in the data that could explain under what circumstances the DRAOR was not used as probation officers adjusted to its use. Also given the novelty of the instrument, it is possible that the accuracy of probation officers in their use of the instrument may have 
been imprecise, particularly in the first few months. While changes in dynamic risk and protective variables were observed over time both within and between offenders, it is also possible that changes in scores could be due to inconsistent application of the tool. There is no information on how probation officers used the tool to structure their contacts with offenders, or whether the DRAOR was being used correctly. Inter-rater reliability is particularly an issue in instruments that include dynamic factors (Latessa \& Lovins, 2010). Inter-rater reliability was not assessed in the current study, so it was not possible to account for the potential influence of parole officer variability.

However, Lowenkamp, Holsinger, Brusman-Lovins, \& Latessa (2004) found high levels of inter-rater reliability between justice personnel immediately following formal training on a risk-needs assessment tool. In addition, the pilot study of the DRAOR found a good overall understanding of most of the DRAOR items by probation officers, with minimal direction required in regards to scoring after the first month of follow-up (Tamatea \& Wilson, 2009). Extra supervision was provided to probation officers after the implementation of the DRAOR, which speaks to the necessity of appropriate training and quality assurance in successfully applying a dynamic risk assessment instrument to offenders (Simourd, 2004). Failing to address these issues may compromise the integrity of the instrument, the accuracy of the assessment for the individual offender, or the resulting management decisions based on the results of the assessment (Bonta, Bogue, Crowley, \& Motiuk, 2001). Of course, inter-rater reliability should be measured in future research given the potential negative consequences if the DRAOR is being applied incorrectly. 


\section{Directions for Future Research}

The DRAOR is a SPJ instrument, but currently there are no guidelines on how to translate scores into decision rules. This study lends support to the need for action when Stable and Acute Risk scores increase and Protective Factor score decrease. Future research will involve developing meaningful cut points with associated decision rules for risk management and frequency of contact. In conjunction with measures of static risk, these decision rules may inform differential supervision for efficient resource allocation. For instance, offenders scoring above a specific score may require a more intensive level of supervision or other interventions. Future research should explore the specificity of decision rules for different subgroups (e.g., sex offenders, domestic violence, women offenders) and different types of violent offenders (e.g., instrumental versus affective).

One aspect of the DRAOR instrument that was left unexamined in the current study was the risk scenario planning. This exercise helps inform the probation officer's structured professional decision making in regards to the level of probation supervision, as well as appropriate interventions and management strategies. Measuring the effects of risk scenario planning was beyond the scope of the current study but remains an important issue for future research. It is important to determine how the scenarios affect decision making, their accuracy in predicting recidivism, and their practical utility.

An additional line of research is the role of the probation officers themselves.

The current study did not address the inter-rater reliability of the DRAOR, but beyond this psychometric issue is how the characteristics of the probation officer influence contacts with offenders. Indeed, the influence of probation officer variability might affect the results of this study in unknown ways. A strong therapeutic alliance may act as 
a protective factor itself, as prior research has found that such a relationship is related to improved offender outcomes, even after controlling for risk (Kennealy, Skeem, Manchak, \& Eno Louden, 2012). The eagerness of probation officers to adopt a new measure of dynamic risk may be related to the perceived utility of the instrument (Bonta \& Wormith, 2007). Based on the pilot study in New Zealand, probation officers were willing to utilize the DRAOR and saw value in its application. The role of the parole or probation officer in assessing and managing risk and acting as effective agents of change are important lines of research to be explored. Inter-rater reliability will need to be examined in the future, as well as the influence of parole officer characteristics (e.g., experience, training) on the administration of the DRAOR.

Replication of the findings of this study are required in other jurisdictions. There are similarities between New Zealand and Canada in terms of the over-representation of Aboriginal peoples, while the United States has a different racial and cultural composition. The validity of the DRAOR with different types of offenders and cultures is an important line of research. The development of web-based training on the DRAOR would facilitate its use by other agencies, and pave the way for extending dynamic risk research.

The current study examined the predictive validity of the DRAOR in comparison to the static risk assessment measure utilized by the New Zealand Department of Corrections (i.e., RoC*RoI). Future research will aim to assess the utility of the DRAOR in relation to other commonly used risk-needs measures, such as the LSI-R (Andrews \& Bonta, 1995). Dynamic risk factors and protective factors were significant predictors of general reconvictions as well as criminal reconvictions in this study. The contribution of 
protective factors to other recidivism outcomes such as sexual offending remains to be tested. In areas such as sexual recidivism where dynamic risk factors are the primary variables in risk assessments (e.g., STABLE-2007, ACUTE-2007), it would be advantageous to examine whether protective factors are incrementally predictive.

Finally, the current study encompassed a measurement-based investigation, and answered valuable questions such as whether the DRAOR is truly dynamic and whether it is equally predictive over time. Ullrich and Coid (2011) also identified a number of conceptual issues regarding the definition and measurement of protective factors, some of which were addressed in this study. Future research should aim to clarify the definition of protective factors at both a theoretical and empirical level to determine how they interact with risk. From a theory of change perspective, a vital question remains whether changes in dynamic risk and protective factors are related to changes in the probability of reconviction or criminal recidivism over time. At a theoretical level, it would be useful to identify distinct trajectories of clusters or groups across time. For instance, "desisters," "persisters," and "no changers" may show different rates of change over time in dynamic risk and protective factors. This may provide insight for parole officers regarding supervision strategies or interventions for offenders showing certain trajectories.

\section{Conclusion}

Consistent with pilot studies of the DRAOR, this study provided support for the DRAOR as an effective measure of risk assessment and risk management. The strengths of this study, namely the large sample and longitudinal design, permitted a multifaceted examination of the relationship of the DRAOR to recidivism. The results of sophisticated analyses suggest this relationship is robust and we can be confident in the DRAOR as a 
decision making tool. In conjunction with its psychometric merit demonstrated in the present research, the findings of this study support the continued clinical use of the DRAOR in New Zealand. 


\section{References}

Andrews, D. A. (1989). Recidivism is predictable and can be influenced: Using risk assessments to reduce recidivism. Forum on Corrections Research, 1(2), 11-18.

Andrews, D. A., \& Bonta, J. (1995). Level of Service Inventory-Revised. Toronto, Canada: Multi-Health Systems.

Andrews, D. A., \& Bonta, J. (2010a). The psychology of criminal conduct (5th ed.). New Providence, NJ: LexisNexis Matthew Bender.

Andrews, D. A., \& Bonta, J. (2010b). Rehabilitating criminal justice policy and practice. Psychology, Public Policy and Law, 16, 39-55. doi: 10.1037/a0018362

Andrews, D. A., Bonta, J., \& Wormith, J. S. (2004). The Level of Service/Case Management Inventory (LS/CMI). Toronto: Multi-Health Systems.

Andrews, D. A., Bonta, J., \& Wormith, J. S. (2006). The recent past and near future of risk and/or needs assessment. Crime \& Delinquency, 52, 7-27. doi: $10.1177 / 0011128705281756$

Andrews, D. A., \& Dowden, C. (2005). Managing correctional treatment for reduced recidivism: A meta-analytic review of program integrity. Legal and Criminological Psychology, 10, 173-187. doi: 10.1348/135532505X36723

Andrews, D. A., \& Wormith, J. S. (1984). Criminal sentiments and criminal behaviour. Programs Branch User Report. Ottawa, Canada: Solicitor General Canada.

Andrews, D. A., Zinger, I., Hoge, R. D., Bonta, J., Gendreau, P., \& Cullen, F. T. (1990). Does correctional treatment work? A clinically-relevant and psychologically informed meta-analysis. Criminology, 28, 369-404. doi: 10.1111/j.17459125.1990.tb01330.x 
Antonowicz, D. H., \& Ross, R. R. (2005). Social problem-solving deficits in offenders. In M. McMurran \& J. McGuire (Eds.), Social problem solving and offending: Evidence, evaluation and evolution (pp. 91-102). New York, NY: John Wiley \& Sons Ltd.

Archer, R. P., Buffington-Vollum, J. K., Stredny, R. V., \& Handel, R. W. (2006). A survey of psychological test use patterns among forensic psychologists. Journal of Personality Assessment, 87, 84-94.

Austin, J., Coleman, D., Peyton, J., \& Johnson, K.D. (2003). Reliability and validity study of the LSI-R risk assessment instrument. Washington, D.C.: Institute on Crime, Justice, and Corrections at The George Washington University.

Ayers, C. D., Williams, J. H., Hawkins, J. D., Peterson, P. L., Catalano, R. F., \& Abbott, R. D. (1999). Assessing correlates of onset, escalation, deescalation, and desistance of delinquent behavior. Journal of Quantitative Criminology, 15(3), 277-306.

Babchishin, K. M., Hanson, R. K., \& Helmus, L. (2012). Even highly correlated measures can add incrementally to predicting recidivism among sex offenders. Assessment, 19, 442-461. doi: 10.1177/1073191112458312

Baird, C. (2009). A question of evidence: A critique of risk assessment models used in the justice system. Madison: WI: National Council on Crime and Delinquency.

Bakker, L., O’Malley, J. \& Riley, D. (1998). Storm warning: Statistical models for predicting violence. Christchurch, New Zealand: Psychological Service, Department of Corrections. 
Bandura, A. (1969). Principles of behaviour modification. Oxford, England: Holt, Rinehart \& Wilson.

Barratt, E. (1994). Impulsiveness and aggression. In J. Monahan \& H. J. Steadman (Eds.), Violence and mental disorder: Developments in risk assessment (pp. 61-79). Chicago: University of Chicago Press.

Beech, A., Friendship, C., Erikson, M., \& Hanson, R. K. (2002). The relationship between static and dynamic risk factors and reconviction in a sample of U.K. child abusers. Sexual Abuse: A Journal of Research and Treatment, 14(2), $155-$ 167. doi: 10.1023/A:1014672231744

Bonta, J. (1996). Risk-needs assessment and treatment. In A. T. Harland (Ed.), Choosing correctional options that work: Defining the demand and evaluating the supply (pp. 18-32). Thousand Oaks, CA: Sage.

Bonta, J., Bogue, B., Crowley, M., \& Motiuk, L. (2001). Implementing offender classification systems: Lessons learned. In G. A. Bernfeld, D. P. Farrington \& A. W. Leschied (Eds.), Offender rehabilitation in practice: Implementing and evaluating effective programs (pp. 227-245). Chichester, England: Wiley.

Bonta, J., Bourgon, G., Rugge, T., Scott, T. L., Yessine, A. K., Gutierrez, L., \& Li, J. (2011). An experimental demonstration of training probation officers in evidencebased community supervision. Criminal Justice and Behavior, 38, 1127-1148. doi: $10.1177 / 0093854811420678$

Bonta, J., Law, M., \& Hanson, R. K. (1998). The prediction of criminal and violent recidivism among mentally disordered offenders: A meta-analysis. Psychological Bulletin, 123, 123-142. doi: 0033-2909/98/S3.00 
Bonta, J., Rugge, T., Scott, T., Bourgon, G., \& Yessine, A. K. (2008). Exploring the black box of community supervision. Journal of Offender Rehabilitation, 47(3), 248-270. doi: 10.1080/10509670802134085

Bonta, J., \& Wormith, S. (2007). Risk and need assessment. In G. McIvor \& P. Raynor (Eds.), Developments in Social Work with Offenders (pp. 131-152). London, England: Jessica Kingsley Publishers.

Boothby, J. L., \& Clements, C. B. (2000). A national survey of correctional psychologists. Criminal Justice and Behaviour, 27(6), 716-732. doi: $10.1177 / 0093854800027006003$

Bourgon, G, and Gutierrez, L. (2012). The general responsivity principle in community supervision: The importance of probation officers using cognitive intervention techniques and its influence on recidivism. Journal of Crime and Justice, 35(2), 149-166. doi: 10.1080/0735648X.2012.674816

Bourgon, G., Gutierrez, L., \& Ashton, J. (2011). From case management to change agent: The evolution of 'What Works' community supervision. Irish Probation Journal, 8, 28-48.

Bourgon, G., Gutierrez, L., \& Ashton, J. (2012). The evolution of community supervision practice: The transformation from case manager to change agent. Federal Probation, 76, 27-35.

Borum, R. (1996). Improving the clinical practice of violence risk assessment: Technology, guidelines, and training. American Psychologist, 51, 945-956.

Borum, R., Bartel, P., \& Forth, A. (2006). Structured Assessment of Violence Risk in Youth (SAVRY). Lutz, FL: Psychological Assessment Resources. 
Borum, R., Lodewijks, H., Bartel, P., \& Forth, A. (2009). Structured Assessment of Violence Risk in Youth (SAVRY). In K. Douglas \& R. Otto (Eds.), Handbook of Violence Risk Assessment (pp. 63-80). New York, NY: Routledge.

Braithwaite, E., Charette, Y., Crocker, A. G., \& Reyes, A. (2010). The predictive validity of clinical ratings of the Short-Term Assessment of Risk and Treatability (START). International Journal of Forensic Mental Health, 9, 271-281. doi: $10.1080 / 14999013.2010 .534378$

Brook, J. S., Whiteman, M., Gordon, A. S., \& Cohen, P. (1989). Changes in drug involvement: A longitudinal study of childhood and adolescent determinants. Psychological Reports, 65(3, Pt 1), 707-726. doi: 10.2466/pr0.1989.65.3.707

Brown, S. L. (2002). The dynamic prediction of criminal recidivism: A three-wave prospective study 1995-2002. Dissertation Abstracts International, 62, $12 \mathrm{~B}$.

Brown, S. L., St. Amand, M. D., \& Zamble, E. (2009). The dynamic prediction of criminal recidivism: A three-year prospective study. Law \& Human Behavior, 33, 25-45. doi: 10.1007/s10979-008-9139-7

Bushway, S. D., Thornberry, T. P., \& Krohn, M. D. (2003). Desistance as a development process: A comparison of static and dynamic approaches. Journal of Quantitative Criminology, 19(2), 129-152. doi: 10.1023/A:1023050103707

Byrne, B. M. (1989). A primer of LISREL: Basic applications and programming for confirmatory factor analytic models. New York: Springer.

Cadsky, O., Hanson, R. K., Crawford, M., \& Lalonde, C. (1996). Attrition from a male batterer treatment program: Client-treatment congruence and lifestyle instability. Violence and Victims, 11, 51-64. 
Campbell, D. T., \& Kenny, D. A. (1999). A primer on regression artifacts. New York: The Guildford Press.

Connors, G. J., Carroll, K. M., DiClemente, C. C., Longabaugh, R., \& Donovan, D. M. (1997). The therapeutic alliance and its relationship to alcoholism treatment participation and outcome. Journal of Consulting and Clinical Psychology, 65(4), 588-598. doi: 10.1037/0022-006X.65.4.588

Costa, F. M., Jessor, R., \& Turbin, M. S. (1999). Transition into adolescent problem drinking: The role of psychosocial risk and protective factors. Journal of Studies on Alcohol, 60, 480-490.

Crits-Christoph, P., Connolly Gibbons, M.B., Hamilton, J., Ring-Kurtz, S., \& Gallop, R. (2011). The dependability of alliance assessments: The alliance-outcome correlation is larger than you might think. Journal of Consulting and Clinical Psychology, 79, 267-278.

Deffenbacher, J. L., Oetting, E. R., \& DiGiuseppe, R. A. (2002). Principles of empirically supported interventions applied to anger management. Counseling Psychologist, 30, 262-280. doi: 10.1177/0011000002302004

Delong, E. R., Delong, D. M., \& Clarke-Pearson, D. L. (1988). Comparing the areas under two or more correlated receiver operating characteristic curves: A nonparametric approach. Biometrics, 44, 837-845.

DeVellis, R. F. (2003). Scale development: Theory and applications (2nd ed.). Thousand Oaks, CA: Sage Publications, Inc. 
de Vogel, V., de Ruiter, C., Bouman, Y., \& de Vries Robbé, M. (2007). Handleiding bij de SAPROF. Structured Assessment of Protective Factors for Violence Risk. Versie 1. [SAPROF Manual. Structured Assessment of Protective Factors for Violence Risk. Version 1]. Utrecht, The Netherlands: Forum Educatief. de Vogel, V., de Ruiter, C., Bouman, Y., \& de Vries Robbé, M. (2009). SAPROF: Guidelines for the assessment of protective factors for violence risk. Utrecht, The Netherlands: Forum Educatief.

de Vries Robbé, M., \& de Vogel, V. (2010). Protective factors for violence risk:

SAPROF prospective results. Paper presented at the tenth Conference of the International Association of Forensic Mental Health Services, Vancouver, Canada.

de Vries Robbé, M., de Vogel, V., Koster, K., \& Bogaerts, S. (in preparation). Protective factors for sexually violent offenders.

de Vries Robbé, M., de Vogel, V., \& de Spa, E. (2011). Protective factors for violence risk in forensic psychiatric patients: A retrospective validation study of the SAPROF. International Journal of Forensic Mental Health, 10, 178-186. doi: $10.1080 / 14999013.2011 .600232$

Douglas, K., \& Skeem, J. (2005). Violence risk assessment: Getting specific about being dynamic. Psychology, Public Policy, \& Law, 11, 347-383. doi: 10.1037/1076-8971.11.3.347

Douglas, K. S., Yeoman, M., \& Boer, D. P. (2005). Comparative validity analysis of multiple measures of violence risk in a sample of criminal offenders. Criminal Justice and Behavior, 32, 479-510. doi: 10.1177/0093854805278411 
Dowden, C., \& Andrews, D. A. (2000). Effective correctional treatment and violent reoffending: A meta-analysis. Canadian Journal of Criminology, 42, 449-467.

Dvoskin, J. A., \& Heilbrun, K. (2001). Risk assessment: Release decision-making toward resolving the great debate. Journal of the American Academy of Psychiatry and the Law, 29(1), 6-10.

Farrington, D. P., \& Loeber, R. (2000). Epidemiology of juvenile violence. Child and Adolescent Psychiatric Clinics of North America, 9, 733-748.

Field, A. (2005). Discovering statistics using SPSS (2nd ed.). London: Sage Publications. Fitzpatrick, K. M. (1997). Fighting among America's youth: A risk and protective factors approach. Journal of Health and Social Behavior, 38, 131-148. doi: $10.2307 / 2955421$

Freese, R., Hiscoke, U., \& Hodgins, S. (2002). The treatment of mentally ill patients with a history of criminality or violence: What works and what doesn't work? Paper presentation at 11th Congress of the Association of European Psychiatrists, Stockholm, Sweden.

Gail, M. H., \& Pfeiffer, R. M. (2005). On criteria for evaluating models of absolute risk. Biostatistics, 6, 227-239. doi: 10.1093/ biostatistics/kxi005

Gendreau, P., Little, T., \& Goggin, C. (1996). A meta-analysis of the predictors of adult offender recidivism: What works! Criminology, 34, 401-433. doi: $10.1111 / \mathrm{j} .1745-9125.1996 . t b 01220 . x$

Gendreau, P., \& Goggin, C. (1996). Principles of effective correctional programming with offenders. Forum on Corrections Research, 8(3), 38-40. 
Griffin, H. L., Beech, A., Print, B., Bradshaw, H., \& Quayle, J. (2008). The development and initial testing of the AIM2 framework to assess risk and strengths in young people who sexually offend. Journal of Sexual Aggression, 14, 211-225. doi: $10.1080 / 13552600802366593$

Green, D., \& Wink, D. (2010). Using random judge assignments to estimate the effects of incarceration and probation on recidivism among drug offenders. Criminology, 48, 357-387. doi: 10.1111/j.1745-9125.2010.00189.x

Grisso, T., \& Appelbaum, P. S. (1992). Is it unethical to offer predictions of future violence? Law and Human Behavior, 16, 621-633. doi: 10.1007/BF01884019.

Grove, W. M., \& Meehl, P. E. (1996). Comparative efficiency of informal (subjective, impressionistic) and formal (mechanical, algorithmic) prediction procedures: The clinical-statistical controversy. Psychology, Public Policy, and Law, 2, 293-323. doi: $10.1037 / 1076-8971.2 .2 .293$

Grubin, D., \& Wingate, S. (1996). Sexual offence recidivism: Prediction versus understanding. Criminal Behavior and Mental Health, 6, 349-359. doi: $10.1002 / \mathrm{cbm} .121$

Hanby, L. J. \& Serin, R. C. (2010) Offender competencies: Understanding change in violent offenders. Poster presentation at the American Psychological Conference. San Diego.

Hanby, L. J., \& Serin, R. C., \& Vuong, B. (2009). Offender competencies and their relationship to correctional program performance. Manuscript submitted for publication. 
Hanson, R. K. (1998). What do we know about sexual offender risk assessment? Psychology, Public Policy and Law, 4, 50-72. doi: 10.1037/1076-8971.4.12.50

Hanson, R. K. (2006). Stability and change: Dynamic risk factors for sexual offenders. In W. L. Marshall, Y. M. Fernandez, L. E. Marshall, \& G. A. Serran (Eds.), Sexual offender treatment: Controversial issues (pp. 17-31). Chichester, UK: John Wiley \& Sons.

Hanson, R. K. (2009). The psychological assessment of risk for crime and violence. Canadian Psychology, 50(3), 172-182. doi : 10.1037/a0015726

Hanson, R. K., \& Harris, A. J. R. (2000). Where should we intervene? Dynamic predictors of sexual offense recidivism. Criminal Justice and Behavior, 27, 6-35. doi: $10.1177 / 0093854800027001002$

Hanson, R. K. \& Harris, A. J. R. (2001). A structured approach to evaluating change among sexual offenders. Sexual Abuse: Journal of Research and Treatment, 13(2), 105-122. doi: 10.1023/A:1026600304489

Hanson, R. K., Harris, A. J. R., Scott, T.-L., \& Helmus, L. (2007). Assessing the risk of sexual offenders on community supervision: The Dynamic Supervision Project. Ottawa, Canada: Public Safety Canada.

Hanson, R. K., \& Morton-Bourgon, K. E. (2009). The accuracy of recidivism risk assessments for sexual offenders: A meta-analysis of 118 prediction studies. Psychological Assessment, 21, 1-21. doi: 10.1037/a0014421 
Harris, P. M., Gingerich, R., \& Whittaker, T. K. (2004). The "effectiveness" of differential supervision. Crime and Delinquency, 50, 235-271. doi: $10.1177 / 0011128703258939$

Hart, S. D., \& Cooke, D. J. (2013). Another look at the (im-)precision of individual risk assessments made using actuarial risk assessment instruments. Behavioral Sciences and the Law, 31, 81-102. doi: 10.1002/bsl.2049

Hawkins, J. D., Catalano, R. F., \& Miller, J. Y. (1992). Risk and protective factors for alcohol and other drug problems in adolescence and early adulthood: Implications for substance abuse prevention. Psychological Bulletin, 112, 64-105. doi: $10.1037 / 0033-2909.112 .1 .64$

Healey, K. M. (1999). Case management in the criminal justice system. Research in Action. Washington, D.C.: National Institute of Justice.

Hosmer, D. W., \& Lemeshow, S. (2000). Applied logistic regression (2nd ed.). New York: John Wiley \& Sons, Inc.

Howells, K. (2004). Anger and its link to violent offending. Psychiatry, Psychology and Law, 11(2), 189-196. doi: 10.1375/1321871042707278

Hudson, S. M., Wales, D. S., Bakker, L., \& Ward, T. (2002). Dynamic risk factors: The Kia Marama evaluation. Sexual Abuse: A Journal of Research and Treatment, 14(2), 103-119. doi: 10.1177/107906320201400203

Hussong, A. M., Curran, P. J., Moffitt, T. E., Caspi, A., \& Carrig, M. M. (2004). Substance abuse hinders desistance in young adults' antisocial behavior. Development and Psychopathology, 16, 1029-1046. 
Johnson, J. L., Lowenkamp, C. T., Vanbenschoten, S. W., \& Robinson, C. R. (2011). The construction and validation of the Federal Post Conviction Risk Assessment (PCRA). Federal Probation, 75(2), 16-29.

Jones, P. R. (1996). Risk prediction in criminal justice. In A. T. Harland (Ed.), Choosing correctional options that work: Defining the demand and evaluating the supply (pp. 33-68). Thousand Oaks, CA: Sage.

Jones, N. J., Brown, S. L., \& Zamble, E. (2010). Predicting criminal recidivism in adult male offenders: Researcher versus parole officer assessment of dynamic risk. Criminal Justice and Behavior, 37(8), 860-882. doi: 10.1177/0093854810368924

Jöreskog, K. G., \& Sörbom, D. (2004). LISREL 8.0 for Windows [Computer Software]. Lincolnwood, IL: Scientific Software International, Inc.

Kazemian, L. (2007). Desistance from crime: Theoretical, empirical, methodological, and policy considerations. Journal of Contemporary Criminal Justice, 23(1), 5-27. doi: $10.1177 / 1043986206298940$

Kennealy, P. J., Skeem, J. L., Manchak, S. M., \& Eno Louden, J. (2012). Firm, fair, and caring officer-offender relationships protect against supervision failure. Law and Human Behavior, 36(6), 496-505. doi: 10.1037/h0093935

Kennedy, S., \& Serin, R. (1999). Examining offender readiness to change and the impact on treatment outcome. In P. M. Harris (Ed.), Research to results: Effective community corrections (pp. 215-232). Arlington, VA: American Correctional Association. 
Kraemer, H. C., Kazdin, A. E., Offord, D. R., Kessler, R. C., Jensen, P. S., \& Kupfer, D. J. (1997). Coming to terms with the terms of risk. Archives of General Psychiatry, $54,337-343$.

Latessa, E. J. (2004). The challenge of change: Correctional programs and evidencebased practices. Criminology and Public Policy, 3, 547-559.

Latessa, E. J., \& Lovins, B. (2010). The role of offender risk assessment: A policy maker guide. Victims and Offenders, 5, 203-219. doi: 10.1080/15564886.2010.485900

Lemieux, C. M. (1998). Determinants of expectation of treatment efficacy among incarcerated substance abusers. International Journal of Offender Therapy and Comparative Criminology, 42(3), 233-245. doi: 10.1177/0306624X9804200305

Lindsay, W. R., Murphy, L., Smith, G., Murphy, D., Edwards, Z., Chittock, C., Grieve, A., \& Young, S. J. (2004). The Dynamic Risk Assessment and Management System: An assessment of immediate risk of violence for individuals with offending and challenging behaviour. Journal of Applied Research in Intellectual Disabilities, 17, 267-274.

Litwack, T. R. (2001). Actuarial versus clinical assessments of dangerousness. Psychology, Public Policy, and Law, 7, 409-443. doi: 10.1037/1076-8971.7.2.409

Lloyd, C. D., \& Serin, R. C. (2012). Agency and outcome expectancies for crime desistance: Measuring offenders' personal beliefs about change. Psychology, Crime \& Law, 18. doi: 10.1080/1068316X.2010.511221 
Lodewijks, H. P. B., Doreleijers, T. A. H., de Ruiter, C., \& Borum, R. (2008). Predictive validity of the Structured Assessment of Violence Risk in Youth (SAVRY) during residential treatment. International Journal of Law and Psychiatry, 31, 263-271. doi: 10.1016/j.ijlp.2008.04.009

Lowenkamp, C. T., Holsinger, A. M., Brusman-Lovins, L., \& Latessa, E. J. (2004). Assessing the inter-rater agreement of the Level of Service Inventory-Revised (Unpublished manuscript). University of Cincinnati, Cincinnati, OH.

Lowenkamp, C. T., \& Latessa, E. J. (2004). Understanding the risk principle: How and why correctional interventions can harm low-risk offenders. In Topics in Community Corrections (pp. 3-8). Washington, DC: National Institute of Corrections.

Lowenkamp, C. T., Pealer, J., Smith, P, \& Latessa, E. J. (2006). Adhering to the risk and need principles: Does it matter for supervision-based programs? Federal Probation, 70(3), 3-8.

Loza, W., \& Loza-Fenous, A. (1999a). Anger and prediction of violent and nonviolent offenders' recidivism. Journal of Interpersonal Violence, 14(10), 1014-1029. doi: $10.1177 / 088626099014010002$

Loza, W., \& Loza-Fenous, A. (1999b). The fallacy of reducing rape and violent recidivism by treating anger. International Journal of Offender Therapy and Comparative Criminology, 43(4), 492-502. doi: 10.1177/0306624X99434007

MacCallum, R. C., Widaman, K. F., Zhang, S., \& Hong S. (1999). Sample size in factor analysis. Psychological Methods, 4, 84-99. 
Mann, R. E., Hanson, R. K., \& Thornton, D. (2010). Assessing risk for sexual recidivism: Some proposals on the nature of psychologically meaningful risk factors. Sexual Abuse: A Journal of Research and Treatment, 22(2), 191-217. doi:10.1177/1079063210366039.

Maruna, S. (2001). Making good: How ex-convicts reform and rebuild their lives. Washington, DC: American Psychological Association Books.

Maume, M. O., Ousey, G. C., \& Beaver, K. (2005). Cutting the grass: A reexamination of the link between marital attachment, delinquent peers and desistance from marijuana use. Journal of Quantitative Criminology, 21, 27-53.

McGuire, J. (2005). Social problem-solving: Basic concepts, research, and applications. In M. McMurran \& J. McGuire (Eds.), Social problem solving and offending: Evidence, evaluation and evolution (pp. 3-30). New York, NY: John Wiley \& Sons Ltd.

McNeil, D. E., Gregory, A. L., Lam, J. N., Binder, R. L., \& Sullivan, G. R., (2003). Utility of decision support tools for assessing acute risk of violence. Journal of Consulting and Clinical Psychology, 71(5), 945-953. doi: 10.1037/0022006X.71.5.945

Mellow, J., \& Christian, J. (2008). Transitioning offenders to the community: A content analysis of reentry guides. Journal of Offender Rehabilitation, 47(4), 339-355. doi: $10.1080 / 10509670801992111$

Miller, H. A. (2006). A dynamic assessment of offender risk, needs, and strengths in a sample of pre-release general offenders. Behavioral Sciences \& the Law, 24, 767782. doi: $10.1002 / b s 1.728$ 
Mills, J. F., \& Kroner, D. G. (2003). Anger as a predictor of institutional misconduct and recidivism in a sample of violent offenders. Journal of Interpersonal Violence, 18(3), 282-294. doi: 10.1177/0886260502250085

Mills, J. F., Kroner, D. G., \& Hemmati, T. (2004). The Measures of Criminal Attitudes and Associates (MCAA): The prediction of general and violent recidivism. Criminal Justice and Behavior, 31(6), 717-733. doi: 10.1177/0093854804268755

Monahan, J., Steadman, H. J., Silver, E., Appelbaum, P. S., Robbins, P. C., Mulvey, E. P., et al. (2001). Rethinking risk assessment: The MacArthur Study of Mental Disorder and Violence. New York: Oxford University Press.

Mossman, D. (1994). Assessing predictions of violence: Being accurate about accuracy. Journal of Consulting and Clinical Psychology, 62, 783-792. doi: 10.1037/0022006X.62.4.783

Motiuk, L. L. (1999). Assessment methods in corrections. In P.M. Harris (Ed.), Research to results: Effective community corrections: Proceedings of the 1995 and 1996 conferences of the International Community Corrections Association (ICCA) (pp. 171-190). Lanham, MD: American Correctional Association.

Nadesu, A. (2009). Reconviction patterns of released prisoners: A 60-months follow-up analysis. New Zealand: Department of Corrections.

Newcomb, M. D., \& Felix-Ortiz, M. (1992). Multiple protective and risk factors for drug use and abuse: Cross-sectional and prospective findings. Journal of Personality and Social Psychology, 63, 280-296. doi: 10.1037/0022-3514.63.2.280 
New Zealand Department of Corrections. (2007). Over-representation of Maori in the criminal justice system: An exploratory report. New Zealand: Department of Corrections.

New Zealand Department of Corrections. (2009). Offender Volumes Report. New Zealand: Department of Corrections.

New Zealand Department of Corrections. (2011). Offender Volumes Report. New Zealand: Department of Corrections.

Nicholls, T. L., Brink, J., Desmarais, S. L., Webster, C. D., \& Martin, M. L. (2006). The Short-Term Assessment of Risk and Treatability (START): A prospective validation study in a forensic psychiatric sample. Assessment, 13, 313-327. doi: $10.1177 / 1073191106290559$

Nonstad, K., Nesset, M. B., Kroppan, E., Pederson, T. W., Nøttestad, J. A., Almvik, R., \& Palmstierna, T. (2010). Predictive validity and other psychometric properties of the Short-Term Assessment of Risk and Treatability (START) in a Norwegian high secure hospital. International Journal of Forensic Mental Health, 9, 294299. doi: $10.1080 / 14999013.2010 .534958$

Nuffield, J. (1989). The SIR scale: Some reflections on its applications. Forum on Corrections Research, 1(2), 19-22.

Nunes, K. L., \& Cortoni, F. (2006). The heterogeneity of treatment non-completers (Research Report No. R-176). Ottawa, Canada: Correctional Service Canada. Parole Act, 2002 No. 10 (2002). 
Pithers, W. D., Kashima, K. M., Cumming, G. F., Beal, L. S., \& Buell, M. M. (1988). Relapse prevention of sexual aggression. Annals of the New York Academy of Sciences, 528, 244-260. doi: 10.1111/j.1749-6632.1988.tb50868.x

Polaschek, D. L. L. (2003). Relapse prevention, offense process models, and the treatment of sexual offenders. Professional Psychology: Research and Practice, 34, 361-367.

Polaschek, D. L. L. \& Collie, R. M. (2004). Rehabilitating serious violent adult offenders: An empirical and theoretical stocktake. Psychology, Crime \& Law, 10(3), 321-334. doi: 10.1080/10683160410001662807

Prentky, R. A., Knight, R. A., Lee., A. F. S., \& Cerce, D. D. (1995). Predictive validity of lifestyle impulsivity for rapists. Criminal Justice and Behavior, 22, 106-128. doi: $10.1177 / 0093854895022002002$

Prochaska, J. O., DiClemente, C. C., \& Norcross, J. (1992). In search of how people change: Applications to addictive behaviors. American Psychologist, 47, 11021114.

Quinsey, V. L., Harris, G. T., Rice, M. E., \& Cormier, C. A. (2006). Violent offenders: Managing and appraising risk (2nd Ed.). Washington, DC: American Psychological Association.

Quinsey, V. L., Jones, G. B., Book, A. S., \& Barr, K. N. (2006). The dynamic prediction of antisocial behavior among forensic psychiatric patients: A prospective field study. Journal of Interpersonal Violence, 21(12), 1539-1565. doi:

$10.1177 / 0886260506294238$ 
Raudenbush, S. W., \& Bryk, A. S. (2011). HLM 7 for Windows [Computer software]. Skokie, IL: Scientific Software International, Inc.

Rice, M. E., \& Harris, G. T. (1995). Violent recidivism: Assessing predictive validity. Journal of Consulting and Clinical Psychology, 63(5), 737-748. doi: 10.1037/0022-006X.63.5.737

Rice, M. E., \& Harris, G. T. (2005). Comparing effect sizes in follow-up studies: ROC Area, Cohen's d, and r. Law and Human Behavior, 29(5), 615-620. doi: $10.1007 / \mathrm{s} 10979-005-6832-7$

Robinson, C. R., VanBenschoten, S., Alexander, M., \& Lowenkamp, C. T. (2011). A random (almost) study of Staff Trained at Reducing Re-arrest (STARR): Reducing recidivism through intentional design. Federal Probation, 75, 57-63.

Robinson, C. R., Lowenkamp, C. T., Holsinger, A. M., VanBenschoten, S., Alexander, M., \& Oleson, J. C. (2012). A random study of Staff Trained at Reducing Rearrest (STARR): Using core correctional in probation interactions. Crime \& Justice, 35, 167-188. doi: 10.1080/0735648X.2012.674823

Rogers, R. (2000). The uncritical acceptance of risk assessment in forensic practice. Law and Human Behavior, 24, 595-605. doi: 10.1023/A: 1005575113507

Rutter, M. (1985). Resilience in the face of adversity: Protective factors and resistance to psychiatric disorder. British Journal of Psychiatry, 147, 598-611. doi:10.1192/bjp.147.6.598

Rutter, M. (1987). Psychosocial resilience and protective mechanisms. American Journal of Orthopsychiatry, 5, 316-331. doi: 10.1111/j.1939-0025.1987.tb03541.x 
Sampson, R. J., \& Laub, J. H. (1990). Crime and deviance over the life course: The salience of adult social bonds. American Sociological Review, 55, 609-627.

Seabloom, W., Seabloom, M. E., Seabloom, E., Barron, R., \& Hendrickson, S. (2003). A 14- to 24-year longitudinal study of a comprehensive sexual health model treatment program for adolescent sex offenders: Predictors of successful completion and subsequent criminal recidivism. International Journal of Offender Therapy and Comparative Criminology, 47, 468-481. doi:

$10.1177 / 0306624 X 03253849$

Serin, R. (2007). The Dynamic Risk Assessment Scale for Offender Re-Entry (DRAOR). Unpublished scale. Carleton University, Ottawa, Ontario.

Serin, R. C., \& Lloyd, C. D. (2009). Examining the process of offender change: The transition to crime desistance. Psychology, Crime, \& Law, 15, 347-364. doi: $10.1080 / 10683160802261078$

Serin, R. C., Lloyd, C., \& Hanby, L. J. (2010). Enhancing offender re-entry: An integrated model for understanding offender re-entry. European Journal of Probation, 2(2), 53-75.

Serin, R. C., Mailloux, D., \& Wilson, N. (2010). Practice manual for use with Dynamic Risk Assessment for Offender Re-entry (DRAOR) scale: Revised version. Unpublished manuscript, Carleton University, Ottawa, Canada.

Serin, R. C. \& Prell, L. (2012). Pathways to crime desistance for probationers. Paper Presented at American Psychology-Law Society Annual Conference, March, San Juan, Puerto Rico. 
Simourd, D. J. (2004). Use of dynamic risk/need assessment instruments among longterm incarcerated offenders. Criminal Justice and Behaviour, 31(3), 306-323. doi: $10.1177 / 0093854803262507$

Singer, J. D. \& Willett, J. B. (2003). Applied longitudinal data analysis: Modelling change and event occurrence. New York: Oxford University Press.

Skeem, J. L., \& Monahan, J. (2011). Current directions in violence risk assessment. Current Directions in Psychological Science, 20, 38-42. doi: $10.1177 / 0963721410397271$

Smith, P., Gendreau, P, \& Swartz, K. (2008). Validating the principles of effective intervention: A systematic review of the contributions of meta-analysis in the field of corrections. Unpublished manuscript, University of Cincinnati, Cincinnati, OH.

Solomon, A., Kachnowski, V., \& Bhati, A. (2005). Does parole work? Analyzing the impact of postprison supervision on rearrest outcomes. Washington, DC: The Urban Institute.

Stübner, S., Groß, G., \& Nedopil, N. (2006). Inpatient risk management with mentally ill offenders: Results of a survey on clinical decision-making about easing restrictions. Criminal Behaviour and Mental Health, 16, 111-123. doi: $10.1002 / \mathrm{cbm} .619$

Swets, J. A. (1986). Indices of discrimination or diagnostic accuracy: Their ROCs and implied models. Psychological Bulletin, 99, 100-117.

Swets, J. A., Dawes, R. M., \& Monahan, J. (2000). Psychological science can improve diagnostic decisions. Psychological Science in the Public Interest: A Journal of the American Psychological Society, 1, 1-26. 
Tabachnick, B. G., \& Fidell, L. S. (2007). Using multivariate statistics (5th ed.). Boston, MA: Pearson Education, Inc.

Tamatea, A., \& Wilson, N. (2009). Dynamic Risk Assessment for Offender Re-entry (DRAOR): A pilot study. New Zealand: Department of Corrections.

Thornton, D. (2002). Constructing and testing a framework for dynamic risk assessment. Sexual Abuse: A Journal of Research and Treatment, 14, 139-153. doi:

$10.1177 / 107906320201400205$

Uggen, C. (1999). Ex-offenders and the conformist alternative: A job quality model of work and crime. Social Problems, 46, 127-151.

Uggen, C. (2000). Work as a turning point in the life course of criminals: A duration model of age, employment, and recidivism. American Sociological Review, 65, $529-546$.

Ullrich, S., \& Coid, J. (2011). Protective factors for violence among released prisoners effects over time and interactions with static risk. Journal of Consulting and Clinical Psychology, 79(3), 381-390. doi: 10.1037/a0023613

Van Voorhis, P. (1997). Correctional classification and the "responsivity principle." Forum on Corrections Research, 9(1), 46-50.

Vincent, G. M., \& Chapman, J., \& Cook, N. E. (2011). Risk-needs assessment in juvenile justice: Predictive validity of the SAVRY, racial differences, and the contribution of needs factors. Criminal Justice and Behavior, 38, 42-62. doi:

$10.1177 / 0093854810386000$ 
Walters, G. D., Trgovac, M., Rychlec, M., DiFazio, R., \& Olson, J. R. (2002). Assessing change with the Psychological Inventory of Criminal Thinking Styles: A controlled analysis and multisite cross-validation. Criminal Justice and Behavior, 29, 308-331. doi: 10.1177/0093854802029003004

Walters, G. D., \& White, T. W. (1989). The thinking criminal: A cognitive model of lifestyle criminality. Criminal Justice Research Bulletin, 4, 1-10.

Ward, T., \& Brown, M. (2004). The good lives model and conceptual issues in offender rehabilitation. Psychology, Crime and Law, 10, 243-257. doi: $10.1080 / 10683160410001662744$

Ward, T., \& Beech, A. R. (2004). The etiology of risk: A preliminary model. Sexual Abuse: A Journal of Research and Treatment, 16(4), 271-284. doi: $10.1177 / 107906320401600402$

Ward, T., Day, A., Howells, K., \& Birgden, A. (2004). The multifactor offender readiness model. Aggression and Violent Behaviour, 9, 645-673. doi: 10.1016/j.avb.2003.08.001

Watson, D., Clark, L. A., \& Tellegen, A. (1988). Development and validation of brief measures of positive and negative affect: The PANAS scales. Journal of Personality and Social Psychology, 54, 1063-1070.

Webster, C. D., Douglas, K. S., Eaves, D., \& Hart, S. D. (1997a). Assessing risk of violence to others. In C. D. Webster \& M. A. Jackson (Eds.), Impulsivity: Theory, assessment, and treatment (pp. 251-277). New York: Guilford. 
Webster, C., Douglas, K., Eaves, D., \& Hart, S. (1997b). HCR-20: Assessing risk for violence (version 2). Burnaby, BC: Mental Health, Law, and Policy Institute, Simon Fraser University.

Webster, C., Martin, M., Brink, J., Nicholls, T., \& Middleton, C. (2004). The Short Term Assessment of Risk and Treatability (START). British Columbia: Forensic Psychiatric Services Commission.

Werner, E. E., \& Smith, R. S. (1992). Overcoming the odds: High-risk children from birth to adulthood. Ithaca, NY: Cornell University Press.

Wong, S. C. P., Olver, M. E., \& Stockdale, K. C. (2009). The utility of dynamic and static factors in risk assessment, prediction, and treatment. In J. T. Andrade (Ed.), Handbook of violence risk assessment and treatment: New approaches for mental health professionals (pp. 83-120). New York, NY: Springer Publishing Co.

Young, S. (2010). Programmed interventions for offenders. In J. M. Brown \& E. A. Campbell (Eds.), The Cambridge Handbook of Forensic Psychology (pp. 384392). New York, NY: Cambridge University Press.

Zamble, E., \& Quinsey, V. L. (1997). The criminal recidivism process. New York: Cambridge University Press.

Zwick, W. R., \& Velicer, W. F. (1986). Comparison of five rules for determining the number of components to retain. Psychological Bulletin, 99(3), 432-442. doi: $10.1037 / 0033-2909.99 .3 .432$ 
Appendices 


\section{Appendix A. Risk of re-Conviction X Risk of re-Imprisonment Model (RoC*RoI)}

Risk of re-Conviction X Risk of re-Imprisonment model. (RoC*RoI; Bakker, O’Malley, \& Riley, 1998).

The $\mathrm{RoC}^{*} \mathrm{RoI}$ measure was developed for the New Zealand Department of Corrections to assist in the accurate prediction of an offender's risk of conviction and likelihood of reimprisonment. The measure is based on static predictors (factors unchangeable by individual effort) from criminal history information. In developing the measure Bakker, O’Malley, and Riley (1999) used the following predictor variables:

\section{Personal characteristics}

- Gender;

- Age (continuous)

- Age at first offence

- Frequency of convictions

- Number of court appearances and convictions (running total)

\section{Jail and time at large}

- Total estimated time (yrs) spent in prison;

- Number of previous imprisonment sentences;

- Indicator that punishment for most recent crime was imprisonment;

- Maximum sentence length handed down to offender in past (yrs);

- Time at large (length of offender's most recent time at large);

\section{Seriousness of offending}

- Sum of seriousness ratings for all crimes (seriousness defined by average length of sentence in days a person receives if convicted of a crime);

- Weighted past seriousness measure (places greater weight on seriousness of most recent offence);

- Maximum serious measures for the past time period;

- Mean seriousness measures for the past time period;

\section{Offence type}

- Offence category (10 possible) (e.g., violent, disorderly conduct, sex);

- Number of convictions in crime category. 


\section{Appendix B. Inter-Correlations between DRAOR Items}

\begin{tabular}{|c|c|c|c|c|c|c|c|c|c|c|c|c|c|c|c|c|c|c|}
\hline & $\mathrm{A} / \mathrm{h}$ & $\mathrm{O} / \mathrm{a}$ & $\mathrm{Nm}$ & Ir & Em & Ls & $\mathrm{Ra}$ & $\mathrm{Pi}$ & $\mathrm{He}$ & $\mathrm{C} / \mathrm{b}$ & Ss & $\mathrm{Sc}$ & $\mathrm{Pa}$ & $\mathrm{Aa}$ & Ic & Ps & $\mathrm{Se}$ & Ao \\
\hline Substance abuse & .31 & .23 & .25 & .22 & .22 & .28 & -.23 & -.28 & -.27 & -.25 & -.25 & -.26 & .34 & .28 & .30 & .26 & .24 & .22 \\
\hline Anger/ hostility $(\mathrm{A} / \mathrm{h})$ & & .22 & .48 & .16 & .23 & .31 & -.26 & -.25 & -.26 & -.25 & -.24 & -.23 & .20 & .34 & .25 & .20 & .29 & .22 \\
\hline $\begin{array}{l}\text { Opportunity/ Access to } \\
\text { victims }(\mathrm{O} / \mathrm{a})\end{array}$ & & & .20 & .16 & .19 & .22 & -.25 & -.28 & -.26 & -.25 & -.26 & -.28 & .28 & .28 & .32 & .29 & .32 & .29 \\
\hline Negative mood (Nm) & & & & .19 & .23 & .29 & -.22 & -.21 & -.23 & -.20 & -.19 & -.19 & .14 & .26 & .22 & .22 & .23 & .22 \\
\hline $\begin{array}{l}\text { Interpersonal } \\
\text { relationships (Ir) }\end{array}$ & & & & & .18 & .21 & -.21 & -.27 & -.25 & -.24 & -.21 & -.23 & .27 & .23 & .24 & .26 & .19 & .17 \\
\hline Employment (Em) & & & & & & .29 & -.25 & -.26 & -.25 & -.23 & -.29 & -.27 & .22 & .23 & .27 & .24 & .22 & .30 \\
\hline Living situation (Ls) & & & & & & & -.24 & -.24 & -.22 & -.23 & -.33 & -.26 & .25 & .26 & .24 & .22 & .20 & .23 \\
\hline $\begin{array}{l}\text { Responsive to advice } \\
\text { (Ra) }\end{array}$ & & & & & & & & .48 & .45 & .49 & .37 & .44 & -.34 & -.46 & -.39 & -.40 & -.42 & -.32 \\
\hline Prosocial identity $(\mathrm{Pi})$ & & & & & & & & & .55 & .49 & .39 & .47 & -.41 & -.41 & -.39 & -.39 & -.39 & -.36 \\
\hline High expectations $(\mathrm{He})$ & & & & & & & & & & .50 & .41 & .44 & -.34 & -.38 & -.36 & -.37 & -.35 & -.36 \\
\hline Costs/benefits $(\mathrm{C} / \mathrm{b})$ & & & & & & & & & & & .36 & .46 & -.34 & -.38 & -.40 & -.43 & -.37 & -.31 \\
\hline Social support (Ss) & & & & & & & & & & & & .49 & -.34 & -.33 & -.31 & -.31 & -.29 & -.36 \\
\hline Social control $(\mathrm{Sc})$ & & & & & & & & & & & & & -.37 & -.36 & -.40 & -.39 & -.36 & -.36 \\
\hline Peer associations $(\mathrm{Pa})$ & & & & & & & & & & & & & & .42 & .39 & .36 & .33 & .30 \\
\hline $\begin{array}{l}\text { Attitudes toward } \\
\text { authority (Aa) }\end{array}$ & & & & & & & & & & & & & & & .43 & .41 & .56 & .42 \\
\hline Impulse control (Ic) & & & & & & & & & & & & & & & & .59 & .43 & .36 \\
\hline Problem solving (Ps) & & & & & & & & & & & & & & & & & .42 & .34 \\
\hline Sense of entitlement (Se) & & & & & & & & & & & & & & & & & & .41 \\
\hline
\end{tabular}

(Se)

Note. Correlations were calculated using Kendall's tau at the last DRAOR assessment $(N=3372)$. All correlations are significant at $p<.001$. 


\section{Appendix C. Exploratory Factor Analysis}

Table 1

Oblique Rotated (Direct Quartimin) Structure Matrix Extracted with Principal Axis

Factoring for DRAOR Items.

\begin{tabular}{lccc}
\hline & \multicolumn{3}{l}{ Factor } \\
\cline { 2 - 4 } & 1 & 2 & 3 \\
\hline Acute - Substance abuse & $\mathbf{. 3 4 5}$ & $\mathbf{. 4 0 7}$ & -.260 \\
Acute - Anger/ hostility & $\mathbf{. 3 6 5}$ & $\mathbf{. 7 8 0}$ & -.285 \\
Acute - Opportunity/ Access to victims & $\mathbf{. 4 3 2}$ & $\mathbf{. 3 1 9}$ & -.329 \\
Acute - Negative mood & .285 & $\mathbf{. 5 9 0}$ & -.247 \\
Acute - Interpersonal relationships & .280 & .149 & -.197 \\
Acute - Employment & .307 & .245 & -.246 \\
Acute - Living situation & .299 & $\mathbf{. 3 5 2}$ & -.299 \\
Protective - Responsive to advice & -.546 & -.345 & $\mathbf{. 6 4 6}$ \\
Protective - Prosocial identity & -.517 & -.317 & $\mathbf{. 7 6 3}$ \\
Protective - High expectations & -.455 & -.326 & $\mathbf{. 7 2 8}$ \\
Protective - Costs/benefits & -.510 & -.285 & $\mathbf{. 7 1 2}$ \\
Protective - Social support & -.410 & -.267 & $\mathbf{. 5 6 8}$ \\
Protective - Social control & -.478 & -.242 & $\mathbf{. 6 8 8}$ \\
Stable - Peer associations & $\mathbf{. 5 2 5}$ & .277 & -.412 \\
Stable - Attitudes toward authority & $\mathbf{. 6 8 1}$ & $\mathbf{. 4 6 8}$ & -.493 \\
Stable - Impulse control & $\mathbf{. 7 1 8}$ & .304 & -.468 \\
Stable - Problem solving & $\mathbf{. 7 0 9}$ & .313 & -.496 \\
Stable - Sense of entitlement & $\mathbf{. 6 8 7}$ & $\mathbf{. 3 7 4}$ & -.464 \\
Stable - Attachment with others & $\mathbf{. 5 6 6}$ & $\mathbf{. 3 5 4}$ & -.441 \\
\hline & & & \\
\hline
\end{tabular}


Table 2

Oblique Rotated (Direct Quartimin) Pattern Matrix Extracted with Principal Axis Factoring for DRAOR Items

\begin{tabular}{lccc} 
& \multicolumn{3}{c}{ Factor } \\
\cline { 2 - 4 } & 1 & 2 & 3 \\
\hline Acute - Substance abuse & .177 & .303 & -.010 \\
Acute - Anger/ hostility & -.015 & .800 & .005 \\
Acute - Opportunity/ Access to victims & .322 & .127 & -.051 \\
Acute - Negative mood & -.031 & .590 & -.036 \\
Acute - Interpersonal relationships & .254 & -.004 & .001 \\
Acute - Employment & .192 & .081 & -.017 \\
Acute - Living situation & .048 & .190 & -.067 \\
Protective - Responsive to advice & -.188 & -.059 & .501 \\
Protective - Prosocial identity & -.017 & -.030 & .760 \\
Protective - High expectations & .075 & -.074 & .754 \\
Protective - Costs/benefits & -.079 & -.004 & .689 \\
Protective - Social support & -.014 & .037 & .426 \\
Protective - Social control & -.050 & .079 & .628 \\
Stable - Peer associations & .434 & -.011 & -.083 \\
Stable - Attitudes toward authority & .556 & .158 & -.042 \\
Stable - Impulse control & .765 & -.033 & -.034 \\
Stable - Problem solving & .711 & -.018 & -.096 \\
Stable - Sense of entitlement & .654 & .039 & -.002 \\
Stable - Attachment with others & .436 & .053 & -.061 \\
\hline
\end{tabular}


Table 3

Oblique Rotated (Direct Quartimin) Structure Matrix Extracted with Generalized Least Squares for DRAOR Items

\begin{tabular}{lccc} 
& \multicolumn{3}{l}{ Factor } \\
\cline { 2 - 4 } & 1 & 2 & 3 \\
\hline Acute - Substance abuse & .312 & -.271 & $\mathbf{. 4 3 4}$ \\
Acute - Anger/ hostility & $\mathbf{. 4 0 9}$ & -.296 & $\mathbf{. 7 3 5}$ \\
Acute - Opportunity/ Access to victims & $\mathbf{. 3 8 7}$ & -.336 & $\mathbf{. 3 3 3}$ \\
Acute - Negative mood & .281 & -.258 & $\mathbf{. 6 3 2}$ \\
Acute - Interpersonal relationships & .249 & -.207 & .161 \\
Acute - Employment & .280 & -.270 & .272 \\
Acute - Living situation & .308 & -.339 & $\mathbf{. 3 8 3}$ \\
Protective - Responsive to advice & -.561 & $\mathbf{. 6 4 3}$ & -.353 \\
Protective - Prosocial identity & -.503 & $\mathbf{. 7 5 5}$ & -.335 \\
Protective - High expectations & -.428 & $\mathbf{. 7 2 9}$ & -.354 \\
Protective - Costs/benefits & -.474 & $\mathbf{. 6 9 5}$ & -.302 \\
Protective - Social support & -.417 & $\mathbf{. 6 0 4}$ & -.322 \\
Protective - Social control & -.455 & $\mathbf{. 7 0 5}$ & -.282 \\
Stable - Peer associations & $\mathbf{. 5 3 4}$ & -.428 & .281 \\
Stable - Attitudes toward authority & $\mathbf{. 7 9 9}$ & -.494 & $\mathbf{. 4 6 4}$ \\
Stable - Impulse control & $\mathbf{. 5 4 6}$ & -.446 & $\mathbf{. 3 2 4}$ \\
Stable - Problem solving & $\mathbf{. 5 3 4}$ & -.474 & $\mathbf{. 3 3 7}$ \\
Stable - Sense of entitlement & $\mathbf{. 7 2 9}$ & -.460 & $\mathbf{. 3 6 6}$ \\
Stable - Attachment with others & $\mathbf{. 5 8 0}$ & -.460 & $\mathbf{. 3 7 1}$ \\
\hline
\end{tabular}


Table 4

Oblique Rotated (Direct Quartimin) Pattern Matrix Extracted with Generalized Least Squares for DRAOR Items

\begin{tabular}{lccc} 
& \multicolumn{3}{c}{ Factor } \\
\cline { 2 - 4 } & 1 & 2 & 3 \\
\hline Acute - Substance abuse & .034 & -.024 & .365 \\
Acute - Anger/ hostility & .100 & .074 & .733 \\
Acute - Opportunity/ Access to victims & .141 & -.079 & .160 \\
Acute - Negative mood & -.090 & .006 & .669 \\
Acute - Interpersonal relationships & .131 & -.051 & .031 \\
Acute - Employment & .090 & -.110 & .153 \\
Acute - Living situation & .083 & -.203 & .276 \\
Protective - Responsive to advice & -.239 & .474 & -.005 \\
Protective - Prosocial identity & -.025 & .731 & .019 \\
Protective - High expectations & .113 & .749 & -.058 \\
Protective - Costs/benefits & -.005 & .646 & .033 \\
Protective - Social support & -.073 & .580 & -.054 \\
Protective - Social control & -.016 & .706 & .053 \\
Stable - Peer associations & .411 & -.134 & -.023 \\
Stable - Attitudes toward authority & .812 & .035 & .068 \\
Stable - Impulse control & .097 & -.012 & .024 \\
Stable - Problem solving & .016 & -.058 & .043 \\
Stable - Sense of entitlement & .686 & .032 & -.022 \\
Stable - Attachment with others & .429 & -.126 & .070 \\
\hline
\end{tabular}




\section{Appendix D. Reliability Analyses of Model \#1 (Original DRAOR)}

Table 1

Item-Total Correlations for Protective Subscale

\begin{tabular}{lcc}
\hline \multicolumn{1}{c}{ Item } & $\begin{array}{c}\text { Corrected Item-Total } \\
\text { Correlation }\end{array}$ & $\begin{array}{c}\text { Cronbach's Alpha if } \\
\text { Item Deleted }\end{array}$ \\
\hline Responsive to advice & .59 & .82 \\
Prosocial identity & .67 & .80 \\
High expectations & .65 & .81 \\
Costs/benefits & .63 & .81 \\
Social support & .52 & .83 \\
Social control & .64 & .81 \\
\hline
\end{tabular}

Note. 6-item scale Cronbach's $\alpha=.84$

Table 2

Item-Total Correlations for Stable Subscale

\begin{tabular}{lcc}
\hline \multicolumn{1}{c}{ Item } & $\begin{array}{c}\text { Corrected Item-Total } \\
\text { Correlation }\end{array}$ & $\begin{array}{c}\text { Cronbach's Alpha if } \\
\text { Item Deleted }\end{array}$ \\
\hline Peer associations & .47 & .80 \\
Attitudes toward authority & .63 & .77 \\
Impulse control & .60 & .78 \\
Problem solving & .60 & .78 \\
Sense of entitlement & .63 & .77 \\
Attachment with others & .51 & .79 \\
\hline
\end{tabular}

Note. 6-item scale Cronbach's $\alpha=.81$

Table 3

Item-Total Correlations for Acute Subscale

\begin{tabular}{lcc}
\hline \multicolumn{1}{c}{ Item } & $\begin{array}{c}\text { Corrected Item-Total } \\
\text { Correlation }\end{array}$ & $\begin{array}{c}\text { Cronbach's Alpha if } \\
\text { Item Deleted }\end{array}$ \\
\hline Substance abuse & .34 & .57 \\
Anger/hostility & .46 & .54 \\
Opportunity/access to victims & .32 & .58 \\
Negative mood & .40 & .56 \\
Interpersonal relationships & .20 & .63 \\
Employment & .27 & .60 \\
Living situation & .36 & .57 \\
\hline
\end{tabular}

Note. 7-item scale Cronbach's $\alpha=.62$ 


\section{Appendix E. Reliability Analyses of Model \#2 of the DRAOR}

Table 1

Item-Total Correlations for Protective Subscale

\begin{tabular}{lcc}
\hline \multicolumn{1}{c}{ Item } & $\begin{array}{c}\text { Corrected Item-Total } \\
\text { Correlation }\end{array}$ & $\begin{array}{c}\text { Cronbach's Alpha if } \\
\text { Item Deleted }\end{array}$ \\
\hline Responsive to advice & .59 & .82 \\
Prosocial identity & .67 & .80 \\
High expectations & .65 & .81 \\
Costs/benefits & .63 & .81 \\
Social support & .52 & .83 \\
Social control & .64 & .81 \\
\hline
\end{tabular}

Note. 6-item scale Cronbach's $\alpha=.84$

Table 2

Item-Total Correlations for Mostly Stable Subscale

\begin{tabular}{lcc}
\hline \multicolumn{1}{c}{ Item } & $\begin{array}{c}\text { Corrected Item-Total } \\
\text { Correlation }\end{array}$ & $\begin{array}{c}\text { Cronbach's Alpha if } \\
\text { Item Deleted }\end{array}$ \\
\hline Peer associations & .48 & .80 \\
Attitudes toward authority & .63 & .78 \\
Impulse control & .59 & .79 \\
Problem solving & .58 & .79 \\
Sense of entitlement & .61 & .78 \\
Attachment with others & .51 & .79 \\
Substance abuse & .37 & .81 \\
Anger/hostility & .41 & .81 \\
Opportunity/access to victims & .41 & .81 \\
\hline
\end{tabular}

Note. 9-item scale Cronbach's $\alpha=81$ 
Table 3

Item-Total Correlations for Mostly Acute Subscale

\begin{tabular}{lcc}
\hline \multicolumn{1}{c}{ Item } & $\begin{array}{c}\text { Corrected Item-Total } \\
\text { Correlation }\end{array}$ & $\begin{array}{c}\text { Cronbach's Alpha if } \\
\text { Item Deleted }\end{array}$ \\
\hline Substance abuse & .36 & .75 \\
Anger/hostility & .51 & .72 \\
Opportunity/access to victims & .39 & .74 \\
Negative mood & .40 & .74 \\
Living situation & .35 & .75 \\
Attitudes toward authority & .57 & .70 \\
Sense of entitlement & .53 & .71 \\
Attachment with others & .49 & .72 \\
\hline
\end{tabular}

Note. 8-item scale Cronbach's $\alpha=.75$ 


\section{Appendix F. Reliability Analyses of Model \#3 of the DRAOR}

Table 1

Item-Total Correlations for Protective Subscale

\begin{tabular}{lcc}
\hline \multicolumn{1}{c}{ Item } & $\begin{array}{c}\text { Corrected Item-Total } \\
\text { Correlation }\end{array}$ & $\begin{array}{c}\text { Cronbach's Alpha if } \\
\text { Item Deleted }\end{array}$ \\
\hline Responsive to advice & .59 & .82 \\
Prosocial identity & .67 & .80 \\
High expectations & .65 & .81 \\
Costs/benefits & .63 & .81 \\
Social support & .52 & .83 \\
Social control & .64 & .81 \\
\hline
\end{tabular}

Note. 6-item scale Cronbach's $\alpha=.84$

Table 2

Item-Total Correlations for Stable Subscale

\begin{tabular}{lcc}
\hline \multicolumn{1}{c}{ Item } & $\begin{array}{c}\text { Corrected Item-Total } \\
\text { Correlation }\end{array}$ & $\begin{array}{c}\text { Cronbach's Alpha if } \\
\text { Item Deleted }\end{array}$ \\
\hline Peer associations & .46 & .80 \\
Attitudes toward authority & .65 & .77 \\
Impulse control & .59 & .78 \\
Problem solving & .59 & .78 \\
Sense of entitlement & .63 & .78 \\
Attachment with others & .52 & .79 \\
Anger/hostility & .38 & .81 \\
Opportunity/access to victims & .41 & .81 \\
\hline
\end{tabular}

Note. 8-item scale Cronbach's $\alpha=.81$ 
Table 3

Item-Total Correlations for Mixed Stable/Acute Subscale

\begin{tabular}{lcc}
\hline \multicolumn{1}{c}{ Item } & $\begin{array}{c}\text { Corrected Item-Total } \\
\text { Correlation }\end{array}$ & $\begin{array}{c}\text { Cronbach's Alpha if } \\
\text { Item Deleted }\end{array}$ \\
\hline Attitudes toward authority & .60 & .77 \\
Impulse control & .55 & .78 \\
Problem solving & .56 & .78 \\
Sense of entitlement & .59 & .77 \\
Attachment with others & .51 & .78 \\
Substance abuse & .37 & .80 \\
Anger/hostility & .48 & .79 \\
Opportunity/access to victims & .42 & .79 \\
Negative mood & .39 & .80 \\
Living situation & .34 & .80 \\
\hline
\end{tabular}

Note. 10-item scale Cronbach's $\alpha=.80$ 


\section{Appendix G. Base Rates of Reconvictions by DRAOR Total Score}

\begin{tabular}{|c|c|c|c|c|c|}
\hline \multirow{2}{*}{$\begin{array}{c}\text { DRAOR } \\
\text { Total Score }\end{array}$} & \multirow[t]{2}{*}{$N$} & \multicolumn{2}{|c|}{ All Reconvictions } & \multicolumn{2}{|c|}{ Criminal Reconvictions } \\
\hline & & $n$ & $\%$ & $n$ & $\%$ \\
\hline-12 & 27 & 5 & 18.5 & 4 & 14.8 \\
\hline-11 & 35 & 4 & 11.4 & 3 & 8.6 \\
\hline-10 & 62 & 7 & 11.3 & 7 & 11.3 \\
\hline-9 & 74 & 12 & 16.2 & 9 & 12.2 \\
\hline-8 & 108 & 27 & 25.0 & 21 & 19.4 \\
\hline-7 & 90 & 21 & 23.3 & 13 & 14.4 \\
\hline-6 & 117 & 22 & 18.8 & 17 & 14.5 \\
\hline-5 & 143 & 48 & 33.6 & 33 & 23.1 \\
\hline-4 & 130 & 45 & 34.6 & 36 & 27.7 \\
\hline-3 & 155 & 30 & 19.4 & 23 & 14.8 \\
\hline-2 & 156 & 46 & 29.5 & 35 & 22.4 \\
\hline-1 & 181 & 63 & 34.8 & 42 & 23.2 \\
\hline 0 & 166 & 51 & 30.7 & 29 & 17.5 \\
\hline 1 & 162 & 61 & 37.7 & 38 & 23.5 \\
\hline 2 & 172 & 74 & 43.0 & 47 & 27.3 \\
\hline 3 & 179 & 77 & 43.0 & 52 & 29.1 \\
\hline 4 & 153 & 78 & 51.0 & 51 & 33.3 \\
\hline 5 & 162 & 82 & 50.6 & 58 & 35.8 \\
\hline 6 & 136 & 69 & 50.7 & 44 & 32.4 \\
\hline 7 & 135 & 68 & 50.4 & 42 & 31.1 \\
\hline 8 & 123 & 76 & 61.8 & 50 & 40.7 \\
\hline 9 & 103 & 70 & 68.0 & 56 & 54.4 \\
\hline 10 & 82 & 58 & 70.7 & 39 & 47.6 \\
\hline 11 & 86 & 63 & 73.3 & 43 & 50.0 \\
\hline 12 & 94 & 74 & 78.7 & 42 & 44.7 \\
\hline 13 & 67 & 41 & 61.2 & 29 & 43.3 \\
\hline 14 & 47 & 34 & 72.3 & 20 & 42.6 \\
\hline 15 & 40 & 30 & 75.0 & 20 & 50.0 \\
\hline 16 & 46 & 32 & 69.6 & 22 & 47.8 \\
\hline 17 & 43 & 35 & 81.4 & 22 & 51.2 \\
\hline 18 & 27 & 21 & 77.8 & 13 & 48.1 \\
\hline 19 & 19 & 16 & 84.2 & 11 & 57.9 \\
\hline 20 & 15 & 14 & 93.3 & 8 & 53.3 \\
\hline 21 & 16 & 12 & 75.0 & 8 & 50.0 \\
\hline 22 & 9 & 7 & 77.8 & 6 & 66.7 \\
\hline 23 & 6 & 6 & 100.00 & 3 & 50.0 \\
\hline 24 & 1 & 1 & 100.00 & 1 & 100.00 \\
\hline 25 & 3 & 2 & 66.7 & 2 & 66.7 \\
\hline 26 & 2 & 2 & 100.00 & 1 & 50.0 \\
\hline
\end{tabular}




\section{Appendix H. Multilevel Models for Change for Reconviction and Criminal Reconviction Predictors}

Table 1

Multilevel Models for Change for Reconviction Predictor Variable

$$
\text { Level-1/Level-2 Specification }
$$

\begin{tabular}{lccc} 
Outcome & Level -1 Model & Composite Model \\
\cline { 2 - 4 } & & & \\
\hline Stable & STABLE $_{\mathrm{ti}}=\pi_{0 \mathrm{i}}+\pi_{\mathrm{ii}} *\left(\mathrm{DAYS}_{\mathrm{ti}}\right)+\mathrm{e}_{\mathrm{ti}}$ & $\pi_{0 \mathrm{i}}=\beta_{00}+\beta_{01} *\left(\mathrm{FAIL}_{\mathrm{i}}\right)+\mathrm{r}_{0 \mathrm{i}}$ & STABLE $_{\mathrm{ti}}=\beta_{00}+\beta_{01} * \mathrm{FAIL}_{\mathrm{i}}+\beta_{10} * \mathrm{DAYS}_{\mathrm{ti}}+\beta_{11} * \mathrm{FAIL}_{\mathrm{i}} * \mathrm{DAYS}_{\mathrm{ti}}$ \\
Risk & & $\pi_{1 \mathrm{i}}=\beta_{10}+\beta_{11} *\left(\mathrm{FAIL}_{\mathrm{i}}\right)+\mathrm{r}_{1 \mathrm{i}}$ & $+\mathrm{r}_{0 \mathrm{i}}+\mathrm{r}_{1 \mathrm{i}} * \mathrm{DAYS}_{\mathrm{ti}}+\mathrm{e}_{\mathrm{ti}}$ \\
Acute & ACUTE $_{\mathrm{ti}}=\pi_{0 \mathrm{i}}+\pi_{\mathrm{li}} *\left(\mathrm{DAYS}_{\mathrm{ti}}\right)+\mathrm{e}_{\mathrm{ti}}$ & $\pi_{0 \mathrm{i}}=\beta_{00}+\beta_{01} *\left(\mathrm{FAIL}_{\mathrm{i}}\right)+\mathrm{r}_{0 \mathrm{i}}$ & ACUTE $_{\mathrm{ti}}=\beta_{00}+\beta_{01} * \mathrm{FAIL}_{\mathrm{i}}+\beta_{10} * \mathrm{DAYS}_{\mathrm{ti}}+\beta_{11} * \mathrm{FAIL}_{\mathrm{i}} * \mathrm{DAYS}_{\mathrm{ti}}$ \\
Risk & & $\pi_{1 \mathrm{i}}=\beta_{10}+\beta_{11} *\left(\mathrm{FAIL}_{\mathrm{i}}\right)+\mathrm{r}_{1 \mathrm{i}}$ & $+\mathrm{r}_{0 \mathrm{i}}+\mathrm{r}_{1 \mathrm{i}} * \mathrm{DAYS}_{\mathrm{ti}}+\mathrm{e}_{\mathrm{ti}}$ \\
Protective & PROTECT $_{\mathrm{ti}}=\pi_{0 \mathrm{i}}+\pi_{\mathrm{ii}} *\left(\mathrm{DAYS}_{\mathrm{ti}}\right)+\mathrm{e}_{\mathrm{ti}}$ & $\pi_{0 \mathrm{i}}=\beta_{00}+\beta_{01} *\left(\mathrm{FAIL}_{\mathrm{i}}\right)+\mathrm{r}_{0 \mathrm{i}}$ & PROTECT $_{\mathrm{ti}}=\beta_{00}+\beta_{01} * \mathrm{FAIL}_{\mathrm{i}}+\beta_{10} * \mathrm{DAYS}_{\mathrm{ti}}+\beta_{11} * \mathrm{FAIL}_{\mathrm{i}} * \mathrm{DAYS}_{\mathrm{ti}}$ \\
Factors & & $\pi_{1 \mathrm{i}}=\beta_{10}+\beta_{11} *\left(\mathrm{FAIL}_{\mathrm{i}}\right)+\mathrm{r}_{1 \mathrm{i}}$ & $+\mathrm{r}_{0 \mathrm{i}}+\mathrm{r}_{1 \mathrm{i}} * \mathrm{DAYS}_{\mathrm{ti}}+\mathrm{e}_{\mathrm{ti}}$
\end{tabular}

Note. These models predict Stable Risk, Acute Risk and Protective Factor in all New Zealand parolees as a function of days since first assessment (at level-1) and all reconvictions categorized into recidivists and non-recidivists (at level-2). The level-2 reconviction predictor is entered in uncentered form. 
Table 2

Multilevel Models for Change for Criminal Reconviction Predictor Variable

\section{Level-1/Level-2 Specification}

\begin{tabular}{|c|c|c|c|}
\hline Outcome & Level -1 Model & Level-2 Model & Composite Model \\
\hline Stable & STABLE $_{\mathrm{ti}}=\pi_{0 \mathrm{i}}+\pi_{\mathrm{li}} *\left(\mathrm{DAYS}_{\mathrm{ti}}\right)+\mathrm{e}_{\mathrm{ti}}$ & $\pi_{0 \mathrm{i}}=\beta_{00}+\beta_{01} *\left(\mathrm{CFAIL}_{\mathrm{i}}\right)+\mathrm{r}_{0 \mathrm{i}}$ & STABLE $_{\mathrm{ti}}=\beta_{00}+\beta_{01} * \mathrm{CFAIL}_{\mathrm{i}}+\beta_{10} * \mathrm{DAYS}_{\mathrm{ti}}+\beta_{11} * \mathrm{CFAIL}_{\mathrm{i}}^{*}$ DAYS $_{\mathrm{ti}}$ \\
\hline Risk & & $\pi_{1 \mathrm{i}}=\beta_{10}+\beta_{11} *\left(\mathrm{CFAIL}_{\mathrm{i}}\right)+\mathrm{r}_{1 \mathrm{i}}$ & $+\mathrm{r}_{0 \mathrm{i}}+\mathrm{r}_{1 \mathrm{i}} * \mathrm{DAYS}_{\mathrm{ti}}+\mathrm{e}_{\mathrm{ti}}$ \\
\hline Acute & $\operatorname{ACUTE}_{\mathrm{ti}}=\pi_{0 \mathrm{i}}+\pi_{1 \mathrm{i}}^{*}\left(\mathrm{DAYS}_{\mathrm{ti}}\right)+\mathrm{e}_{\mathrm{ti}}$ & $\pi_{0 \mathrm{i}}=\beta_{00}+\beta_{01} *\left(\mathrm{CFAIL}_{\mathrm{i}}\right)+\mathrm{r}_{0 \mathrm{i}}$ & ACUTE $_{\mathrm{ti}}=\beta_{00}+\beta_{01} *$ CFAIL $_{\mathrm{i}}+\beta_{10} * \mathrm{DAYS}_{\mathrm{ti}}+\beta_{11} * \mathrm{CFAIL}_{\mathrm{i}} * \mathrm{DAYS}_{\mathrm{ti}}$ \\
\hline Risk & & $\pi_{1 \mathrm{i}}=\beta_{10}+\beta_{11} *\left(\mathrm{CFAIL}_{\mathrm{i}}\right)+\mathrm{r}_{1 \mathrm{i}}$ & $+\mathrm{r}_{0 \mathrm{i}}+\mathrm{r}_{1 \mathrm{i}} * \mathrm{DAYS} \mathrm{S}_{\mathrm{ti}}+\mathrm{e}_{\mathrm{ti}}$ \\
\hline Protective & PROTECT $_{\mathrm{ti}}=\pi_{0 \mathrm{i}}+\pi_{1 \mathrm{i}} *\left(\mathrm{DAYS}_{\mathrm{ti}}\right)$ & $\pi_{0 \mathrm{i}}=\beta_{00}+\beta_{01} *\left(\mathrm{CFAIL}_{\mathrm{i}}\right)+\mathrm{r}_{0 \mathrm{i}}$ & PROTECT $_{\mathrm{ti}}=\beta_{00}+\beta_{01} * \mathrm{CFAIL}_{\mathrm{i}}+\beta_{10} *$ DAYS $_{\mathrm{ti}}+\beta_{11} *$ CFAIL $_{\mathrm{i}} *$ DAYS $_{\mathrm{ti}}$ \\
\hline Factors & $+e_{t i}$ & $\pi_{1 \mathrm{i}}=\beta_{10}+\beta_{11} *\left(\right.$ CFAIL $\left._{\mathrm{i}}\right)+\mathrm{r}_{1 \mathrm{i}}$ & $+r_{0 i}+r_{1 i} * D_{A Y S}+e_{t i}$ \\
\hline
\end{tabular}

Note. These models predict Stable Risk, Acute Risk and Protective Factor in all New Zealand parolees as a function of days since first assessment (at level-1) and all criminal reconvictions categorized into recidivists and non-recidivists (at level-2). The level-2 criminal reconviction predictor is entered in uncentered form. 


\section{Appendix I. Multilevel Models for Change for Timing of DRAOR Assessments}

Table 1

Multilevel Models for Change Fitted to 12-Month Stable Risk Data

\begin{tabular}{|c|c|c|c|}
\hline \multirow[b]{2}{*}{ Outcome } & \multicolumn{2}{|c|}{ Level-1/Level-2 Specification } & \multirow[b]{2}{*}{ Composite Model } \\
\hline & Level -1 Model & Level-2 Model & \\
\hline Unconditional & $\begin{array}{l}\operatorname{STABLE}_{\mathrm{ti}}=\pi_{0 \mathrm{i}}+\pi_{1 \mathrm{i}} *\left(\mathrm{MONTH}_{\mathrm{ti}}\right) \\
+\pi_{2 \mathrm{i}} *\left(\mathrm{MONTH}_{\mathrm{ti}}\right)\end{array}$ & $\begin{aligned} \pi_{0 \mathrm{i}} & =\beta_{00}+\mathrm{r}_{0 \mathrm{i}} \\
\pi_{1 \mathrm{i}} & =\beta_{10}\end{aligned}$ & $\begin{array}{l}\text { STABLE }_{\mathrm{ti}}=\beta_{00}+\beta_{10} * \mathrm{MONTH}_{\mathrm{ti}}+\beta_{20} * \mathrm{MONTH}_{\mathrm{ti}}+\beta_{30} * \mathrm{MONTH}_{\mathrm{ti}} \\
\quad+\beta_{40} * \mathrm{MONTH}_{\mathrm{ti}}+\beta_{50} * \mathrm{MONTH}_{\mathrm{ti}}+\beta_{60} * \mathrm{MONTH}_{\mathrm{ti}}\end{array}$ \\
\hline \multirow[t]{10}{*}{ Growth } & $+\pi_{3 \mathrm{i}} *\left(\mathrm{MONTH}_{\mathrm{ti}}\right)$ & $\pi_{2 \mathrm{i}}=\beta_{20}$ & $+\beta_{70} * \mathrm{MONTH}_{\mathrm{ti}}+\beta_{80} * \mathrm{MONTH} 9_{\mathrm{ti}}+\beta_{90} * \mathrm{MONTH} 10_{\mathrm{ti}}$ \\
\hline & $+\pi_{4 \mathrm{i}} *\left(\mathrm{MONTH}_{\mathrm{ti}}\right)$ & $\pi_{3 \mathrm{i}}=\beta_{30}$ & $+\beta_{100} *$ MONTH1 $11_{\mathrm{ti}}+\beta_{110} *$ MONTH12 ${ }_{\mathrm{ti}}+\mathrm{r}_{0 \mathrm{i}}+\mathrm{e}_{\mathrm{ti}}$ \\
\hline & $+\pi_{5 \mathrm{i}}{ }^{*}\left(\mathrm{MONTH}_{\mathrm{ti}}\right)$ & $\pi_{4 \mathrm{i}}=\beta_{40}$ & \\
\hline & $+\pi_{6 \mathrm{i}} *\left(\mathrm{MONTH} 7_{\mathrm{ti}}\right)$ & $\pi_{5 \mathrm{i}}=\beta_{50}$ & \\
\hline & $+\pi_{7 \mathrm{i}} *\left(\mathrm{MONTH} 8_{\mathrm{ti}}\right)$ & $\pi_{6 \mathrm{i}}=\beta_{60}$ & \\
\hline & $+\pi_{8 \mathrm{i}} *\left(\mathrm{MONTH} 9_{\mathrm{ti}}\right)$ & $\pi_{7 \mathrm{i}}=\beta_{70}$ & \\
\hline & $+\pi_{9 \mathrm{i}} *\left(\mathrm{MONTH} 10_{\mathrm{ti}}\right)$ & $\pi_{8 \mathrm{i}}=\beta_{80}$ & \\
\hline & $+\pi_{10 \mathrm{i}} *\left(\mathrm{MONTH} 11_{\mathrm{ti}}\right)$ & $\pi_{9 \mathrm{i}}=\beta_{90}$ & \\
\hline & $+\pi_{11 \mathrm{i}} *\left(\mathrm{MONTH} 12_{\mathrm{ti}}\right)+\mathrm{e}_{\mathrm{ti}}$ & $\pi_{10 \mathrm{i}}=\beta_{100}$ & \\
\hline & & $\pi_{11 \mathrm{i}}=\beta_{110}$ & \\
\hline \multirow[t]{12}{*}{ Reconvictions } & $\mathrm{STABLE}_{\mathrm{ti}}=\pi_{0 \mathrm{i}}+\pi_{1 \mathrm{i}} *\left(\mathrm{MONTH}_{\mathrm{ti}}\right)$ & $\pi_{0 \mathrm{i}}=\beta_{00}+\beta_{01} *\left(\mathrm{FAIL}_{\mathrm{i}}\right)+\mathrm{r}_{0 \mathrm{i}}$ & $\mathrm{STABLE}_{\mathrm{ti}}=\beta_{00}+\beta_{01} * \mathrm{FAIL}_{\mathrm{i}}$ \\
\hline & $+\pi_{2 \mathrm{i}} *\left(\mathrm{MONTH} 3_{\mathrm{ti}}\right)$ & $\pi_{1 \mathrm{i}}=\beta_{10}+\beta_{11} *\left(\mathrm{FAIL}_{\mathrm{i}}\right)$ & $+\beta_{10} * \mathrm{MONTH} 2_{\mathrm{ti}}+\beta_{11} * \mathrm{FAIL}_{\mathrm{i}} * \mathrm{MONTH}_{\mathrm{ti}}$ \\
\hline & $+\pi_{3 \mathrm{i}} *\left(\mathrm{MONTH}_{\mathrm{ti}}\right)$ & $\pi_{2 \mathrm{i}}=\beta_{20}+\beta_{21} *\left(\mathrm{FAIL}_{\mathrm{i}}\right)$ & $+\beta_{20} * \mathrm{MONTH}_{\mathrm{ti}}+\beta_{21} * \mathrm{FAIL}_{\mathrm{i}} * \mathrm{MONTH}_{\mathrm{ti}}$ \\
\hline & $+\pi_{4 \mathrm{i}} *\left(\mathrm{MONTH}_{\mathrm{ti}}\right)$ & $\pi_{3 \mathrm{i}}=\beta_{30}+\beta_{31} *\left(\mathrm{FAIL}_{\mathrm{i}}\right)$ & $+\beta_{30} * \mathrm{MONTH}_{\mathrm{ti}}+\beta_{31} * \mathrm{FAIL}_{\mathrm{i}} * \mathrm{MONTH}_{\mathrm{ti}}$ \\
\hline & $+\pi_{5 \mathrm{i}} *\left(\mathrm{MONTH6}_{\mathrm{ti}}\right)$ & $\pi_{4 \mathrm{i}}=\beta_{40}+\beta_{41} *\left(\mathrm{FAIL}_{\mathrm{i}}\right)$ & $+\beta_{40} *$ MONTH $_{\mathrm{ti}}+\beta_{41} *$ FAIL $_{\mathrm{i}} *$ MONTH $_{\mathrm{ti}}$ \\
\hline & $+\pi_{6 \mathrm{i}} *\left(\mathrm{MONTH} 7_{\mathrm{ti}}\right)$ & $\pi_{5 \mathrm{i}}=\beta_{50}+\beta_{51} *\left(\mathrm{FAIL}_{\mathrm{i}}\right)$ & $+\beta_{50} *$ MONTH6 $_{\mathrm{ti}}+\beta_{51} *$ FAIL $_{\mathrm{i}} *$ MONTH6 $_{\mathrm{ti}}$ \\
\hline & $+\pi_{7 \mathrm{i}} *\left(\mathrm{MONTH} 8_{\mathrm{ti}}\right)$ & $\pi_{6 \mathrm{i}}=\beta_{60}+\beta_{61} *\left(\mathrm{FAIL}_{\mathrm{i}}\right)$ & $+\beta_{60} * \mathrm{MONTH}_{\mathrm{ti}}+\beta_{61} * \mathrm{FAIL}_{\mathrm{i}} * \mathrm{MONTH}_{\mathrm{ti}}$ \\
\hline & $+\pi_{8 \mathrm{i}} *\left(\mathrm{MONTH} 9_{\mathrm{ti}}\right)$ & $\pi_{7 \mathrm{i}}=\beta_{70}+\beta_{71} *\left(\mathrm{FAIL}_{\mathrm{i}}\right)$ & $+\beta_{70} * \mathrm{MONTH}_{\mathrm{ti}}+\beta_{71} * \mathrm{FAIL}_{\mathrm{i}} * \mathrm{MONTH}_{\mathrm{ti}}$ \\
\hline & $+\pi_{9 \mathrm{i}} *\left(\mathrm{MONTH} 10_{\mathrm{ti}}\right)$ & $\pi_{8 \mathrm{i}}=\beta_{80}+\beta_{81} *\left(\mathrm{FAIL}_{\mathrm{i}}\right)$ & $+\beta_{80} * \mathrm{MONTH}_{\mathrm{ti}}+\beta_{81} * \mathrm{FAIL}_{\mathrm{i}} * \mathrm{MONTH} 9_{\mathrm{ti}}$ \\
\hline & $+\pi_{10 \mathrm{i}} *\left(\mathrm{MONTH} 11_{\mathrm{ti}}\right)$ & $\pi_{9 \mathrm{i}}=\beta_{90}+\beta_{91} *\left(\mathrm{FAIL}_{\mathrm{i}}\right)$ & $+\beta_{90} *$ MONTH10 $0_{\mathrm{ti}}+\beta_{91} * \mathrm{FAIL}_{\mathrm{i}} * \mathrm{MONTH}_{10} 0_{\mathrm{ti}}$ \\
\hline & $+\pi_{11 \mathrm{i}} *\left(\mathrm{MONTH} 12_{\mathrm{ti}}\right)+\mathrm{e}_{\mathrm{ti}}$ & $\pi_{10 \mathrm{i}}=\beta_{100}+\beta_{101} *\left(\mathrm{FAIL}_{\mathrm{i}}\right)$ & $+\beta_{100} *$ MONTH11 $1_{\mathrm{ti}}+\beta_{101} *$ FAIL $_{\mathrm{i}} *$ MONTH11 $1_{\mathrm{ti}}$ \\
\hline & & $\pi_{11 \mathrm{i}}=\beta_{110}+\beta_{111} *\left(\mathrm{FAIL}_{\mathrm{i}}\right)$ & $\begin{array}{l}+\beta_{110} * \text { MONTH12 }{ }_{\mathrm{ti}}+\beta_{111} * \mathrm{FAIL}_{\mathrm{i}} * \mathrm{MONTH} 12_{\mathrm{ti}} \\
+\mathrm{r}_{0 \mathrm{i}}+\mathrm{e}_{\mathrm{ti}}\end{array}$ \\
\hline
\end{tabular}




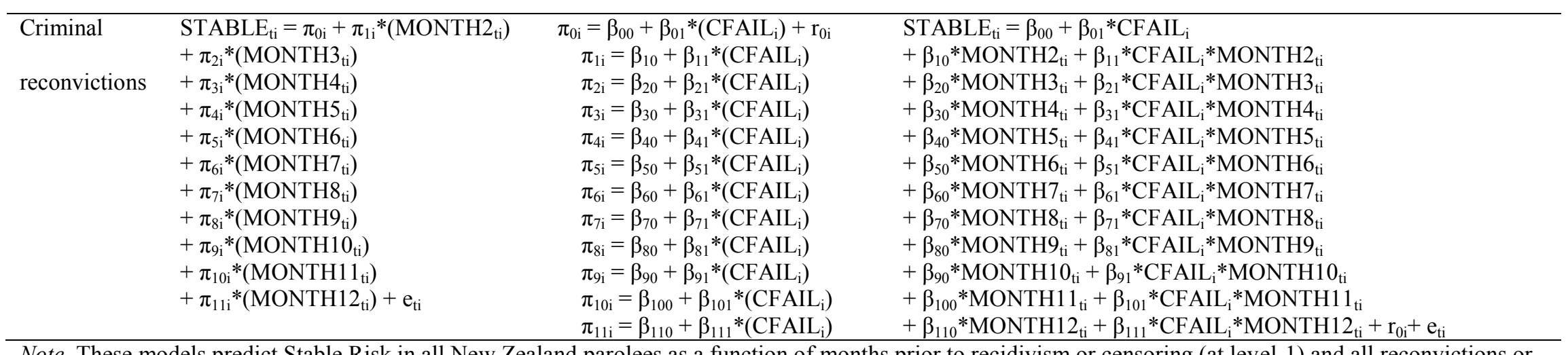

criming models predict Stable Risk in all New Zealand parolees as a function of months prior to recidivism or censoring (at level-1) and all reconvi 
Table 2

Multilevel Models for Change Fitted to 12-Month Acute Risk Data

$$
\text { Level-1/Level-2 Specification }
$$

\begin{tabular}{|c|c|c|c|}
\hline Outcome & Level -1 Model & Level-2 Model & Composite Model \\
\hline Unconditional & 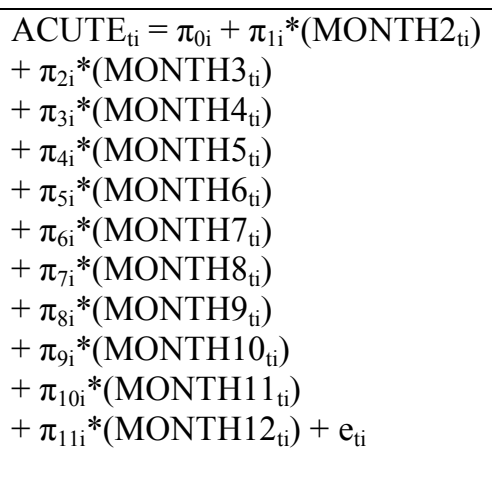 & $\begin{array}{l}\pi_{0 \mathrm{i}}=\beta_{00}+\mathrm{r}_{0 \mathrm{i}} \\
\pi_{1 \mathrm{i}}=\beta_{10} \\
\pi_{2 \mathrm{i}}=\beta_{20} \\
\pi_{3 \mathrm{i}}=\beta_{30} \\
\pi_{4 \mathrm{i}}=\beta_{40} \\
\pi_{5 \mathrm{i}}=\beta_{50} \\
\pi_{6 \mathrm{i}}=\beta_{60} \\
\pi_{7 \mathrm{i}}=\beta_{70} \\
\pi_{8 \mathrm{i}}=\beta_{80} \\
\pi_{9 \mathrm{i}}=\beta_{90} \\
\pi_{10 \mathrm{i}}=\beta_{100} \\
\pi_{11 \mathrm{i}}=\beta_{110}\end{array}$ & $\begin{array}{l}\text { ACUTE }_{\mathrm{ti}}=\beta_{00}+\beta_{10} * \mathrm{MONTH}_{\mathrm{ti}}+\beta_{20} * \mathrm{MONTH}_{\mathrm{ti}}+\beta_{30} * \mathrm{MONTH}_{\mathrm{ti}} \\
\quad+\beta_{40} * \text { MONTH } 5_{\mathrm{ti}}+\beta_{50} * \mathrm{MONTH}_{\mathrm{ti}}+\beta_{60} * \mathrm{MONTH} 7_{\mathrm{ti}} \\
\quad+\beta_{70} * \text { MONTH } 8_{\mathrm{ti}}+\beta_{80} * \text { MONTH } 9_{\mathrm{ti}}+\beta_{90} * \mathrm{MONTH} 10_{\mathrm{ti}} \\
\quad+\beta_{100} * \text { MONTH } 11_{\mathrm{ti}}+\beta_{110} * \mathrm{MONTH} 12_{\mathrm{ti}}+\mathrm{r}_{0 \mathrm{i}}+\mathrm{e}_{\mathrm{ti}}\end{array}$ \\
\hline Reconvictions & 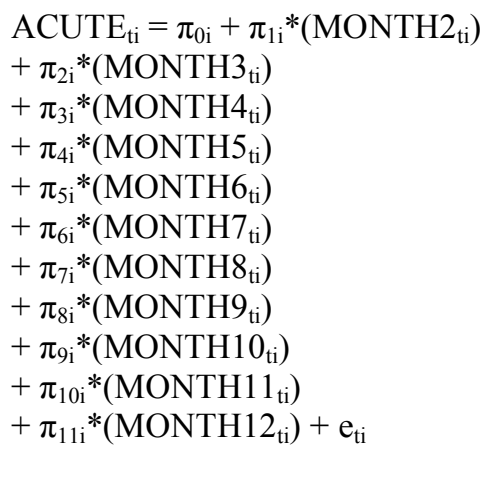 & $\begin{aligned} \pi_{0 \mathrm{i}}=\beta_{00}+\beta_{01} *\left(\mathrm{FAIL}_{\mathrm{i}}\right)+\mathrm{r}_{0 \mathrm{i}} \\
\pi_{1 \mathrm{i}}=\beta_{10}+\beta_{11} *\left(\mathrm{FAIL}_{\mathrm{i}}\right) \\
\pi_{2 \mathrm{i}}=\beta_{20}+\beta_{21} *\left(\mathrm{FAIL}_{\mathrm{i}}\right) \\
\pi_{3 \mathrm{i}}=\beta_{30}+\beta_{31} *\left(\mathrm{FAIL}_{\mathrm{i}}\right) \\
\pi_{4 \mathrm{i}}=\beta_{40}+\beta_{41} *\left(\mathrm{FAIL}_{\mathrm{i}}\right) \\
\pi_{5 \mathrm{i}}=\beta_{50}+\beta_{51} *\left(\mathrm{FAIL}_{\mathrm{i}}\right) \\
\pi_{6 \mathrm{i}}=\beta_{60}+\beta_{61} *\left(\mathrm{FAIL}_{\mathrm{i}}\right) \\
\pi_{7 \mathrm{i}}=\beta_{70}+\beta_{71} *\left(\mathrm{FAIL}_{\mathrm{i}}\right) \\
\pi_{8 \mathrm{i}}=\beta_{80}+\beta_{81} *\left(\mathrm{FAIL}_{\mathrm{i}}\right) \\
\pi_{9 \mathrm{i}}=\beta_{90}+\beta_{91} *\left(\mathrm{FAIL}_{\mathrm{i}}\right) \\
\pi_{10 \mathrm{i}}=\beta_{100}+\beta_{101} *\left(\mathrm{FAIL}_{\mathrm{i}}\right) \\
\pi_{11 \mathrm{i}}=\beta_{110}+\beta_{111} *\left(\mathrm{FAIL}_{\mathrm{i}}\right)\end{aligned}$ & 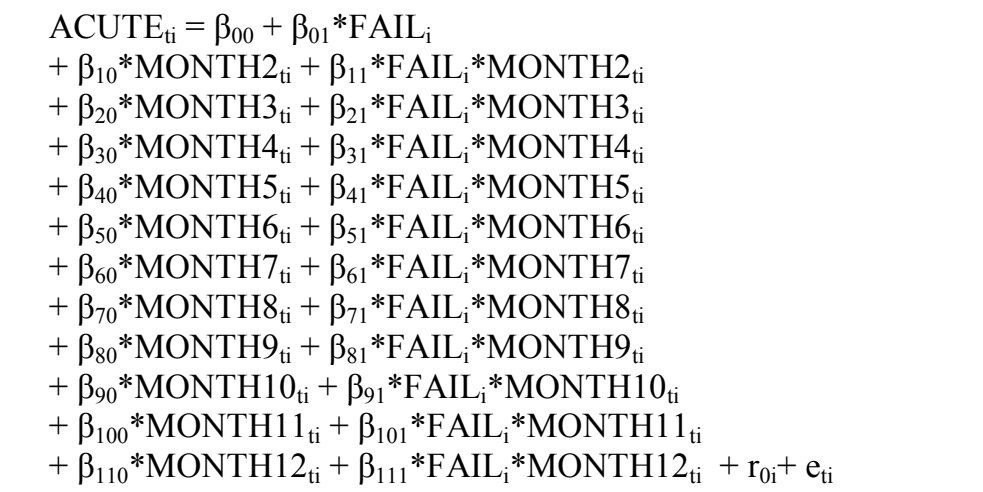 \\
\hline
\end{tabular}




\begin{tabular}{|c|c|c|c|}
\hline Criminal & $\begin{array}{l}\text { ACUTE }_{\mathrm{ti}}=\pi_{0 \mathrm{i}}+\pi_{1 \mathrm{i}} *\left(\mathrm{MONTH}_{\mathrm{ti}}\right) \\
+\pi_{2 \mathrm{i}} *\left(\mathrm{MONTH}_{\mathrm{ti}}\right)\end{array}$ & $\begin{array}{c}\pi_{0 \mathrm{i}}=\beta_{00}+\beta_{01} *\left(\mathrm{CFAIL}_{\mathrm{i}}\right)+\mathrm{r}_{0 \mathrm{i}} \\
\pi_{1 \mathrm{i}}=\beta_{10}+\beta_{11} *\left(\mathrm{CFAIL}_{\mathrm{i}}\right)\end{array}$ & $\begin{array}{l}\text { ACUTE }_{\mathrm{ti}}=\beta_{00}+\beta_{01} * \mathrm{CFAIL}_{\mathrm{i}} \\
+\beta_{10} * \text { MONTH } 2_{\mathrm{ti}}+\beta_{11} * \text { CFAIL }_{\mathrm{i}} * \text { MONTH } 2_{\mathrm{ti}}\end{array}$ \\
\hline \multirow[t]{10}{*}{ reconvictions } & $+\pi_{3 \mathrm{i}} *\left(\mathrm{MONTH}_{\mathrm{ti}}\right)$ & $\pi_{2 \mathrm{i}}=\beta_{20}+\beta_{21} *\left(\mathrm{CFAIL}_{\mathrm{i}}\right)$ & $+\beta_{20} * \mathrm{MONTH}_{\mathrm{ti}}+\beta_{21} * \mathrm{CFAIL}_{\mathrm{i}} * \mathrm{MONTH}_{\mathrm{ti}}$ \\
\hline & $+\pi_{4 \mathrm{i}} *\left(\mathrm{MONTH}_{\mathrm{ti}}\right)$ & $\pi_{3 \mathrm{i}}=\beta_{30}+\beta_{31} *\left(\mathrm{CFAIL}_{\mathrm{i}}\right)$ & $+\beta_{30} * \mathrm{MONTH}_{\mathrm{ti}}+\beta_{31} * \mathrm{CFAIL}_{\mathrm{i}} * \mathrm{MONTH}_{\mathrm{ti}}$ \\
\hline & $+\pi_{5 \mathrm{i}} *\left(\mathrm{MONTH}_{\mathrm{ti}}\right)$ & $\pi_{4 \mathrm{i}}=\beta_{40}+\beta_{41} *\left(\mathrm{CFAIL}_{\mathrm{i}}\right)$ & $+\beta_{40} * \mathrm{MONTH}_{\mathrm{ti}}+\beta_{41} * \mathrm{CFAIL}_{\mathrm{i}} * \mathrm{MONTH}_{\mathrm{ti}}$ \\
\hline & $+\pi_{6 \mathrm{i}} *\left(\mathrm{MONTH} 7_{\mathrm{ti}}\right)$ & $\pi_{5 \mathrm{i}}=\beta_{50}+\beta_{51} *\left(\mathrm{CFAIL}_{\mathrm{i}}\right)$ & $+\beta_{50} * \mathrm{MONTH}_{\mathrm{ti}}+\beta_{51} * \mathrm{CFAIL}_{\mathrm{i}} * \mathrm{MONTH}_{\mathrm{ti}}$ \\
\hline & $+\pi_{7 \mathrm{i}} *\left(\mathrm{MONTH} 8_{\mathrm{ti}}\right)$ & $\pi_{6 \mathrm{i}}=\beta_{60}+\beta_{61} *\left(\mathrm{CFAIL}_{\mathrm{i}}\right)$ & $+\beta_{60} * \mathrm{MONTH}_{\mathrm{ti}}+\beta_{61} * \mathrm{CFAIL}_{\mathrm{i}} * \mathrm{MONTH}_{\mathrm{ti}}$ \\
\hline & $+\pi_{8 \mathrm{i}} *\left(\mathrm{MONTH} 9_{\mathrm{ti}}\right)$ & $\pi_{7 \mathrm{i}}=\beta_{70}+\beta_{71} *\left(\mathrm{CFAIL}_{\mathrm{i}}\right)$ & $+\beta_{70} * \mathrm{MONTH}_{\mathrm{ti}}+\beta_{71} * \mathrm{CFAIL}_{\mathrm{i}} * \mathrm{MONTH} 8_{\mathrm{ti}}$ \\
\hline & $+\pi_{9 \mathrm{i}} *\left(\mathrm{MONTH} 10_{\mathrm{ti}}\right)$ & $\pi_{8 \mathrm{i}}=\beta_{80}+\beta_{81} *\left(\mathrm{CFAIL}_{\mathrm{i}}\right)$ & $+\beta_{80} *$ MONTH $9_{\mathrm{ti}}+\beta_{81} * \mathrm{CFAIL}_{\mathrm{i}} * \mathrm{MONTH}_{\mathrm{ti}}$ \\
\hline & $+\pi_{10 \mathrm{i}} *\left(\mathrm{MONTH} 11_{\mathrm{ti}}\right)$ & $\pi_{9 \mathrm{i}}=\beta_{90}+\beta_{91} *\left(\mathrm{CFAIL}_{\mathrm{i}}\right)$ & $+\beta_{90} *$ MONTH $10_{\mathrm{ti}}+\beta_{91} * \mathrm{CFAIL}_{\mathrm{i}}^{*} \mathrm{MONTH}_{10} 0_{\mathrm{ti}}$ \\
\hline & $+\pi_{11 \mathrm{i}} *\left(\mathrm{MONTH} 12_{\mathrm{ti}}\right)+\mathrm{e}_{\mathrm{ti}}$ & $\pi_{10 \mathrm{i}}=\beta_{100}+\beta_{101} *\left(\mathrm{CFAIL}_{\mathrm{i}}\right)$ & $+\beta_{100} *$ MONTH1 $11_{\mathrm{ti}}+\beta_{101} * \mathrm{CFAIL}_{\mathrm{i}}^{*} \mathrm{MONTH}_{1} 1_{\mathrm{ti}}$ \\
\hline & & $\pi_{11 \mathrm{i}}=\beta_{110}+\beta_{111} *\left(\mathrm{CFAIL}_{\mathrm{i}}\right)$ & $+\beta_{110} *$ MONTH12 $2_{\mathrm{ti}}+\beta_{111} * \mathrm{CFAIL}_{\mathrm{i}}^{*} \mathrm{MONTH}_{12} 2_{\mathrm{ti}}+\mathrm{r}_{0 \mathrm{i}}+\mathrm{e}_{\mathrm{ti}}$ \\
\hline
\end{tabular}

Note. These models predict Acute Risk in all New Zealand parolees as a function of months prior to recidivism or censoring (at level-1) and all reconvictions or criminal reconvictions categorized into recidivists and non-recidivists (at level-2). The level-2 reconviction predictor is entered in uncentered form. 
Table 3

Multilevel Models for Change Fitted to 12-Month Protective Factors Data

Level-1/Level-2 Specification

\begin{tabular}{|c|c|c|c|}
\hline Outcome & Level -1 Model & Level-2 Model & Composite Model \\
\hline $\begin{array}{l}\text { Unconditional } \\
\text { Growth }\end{array}$ & 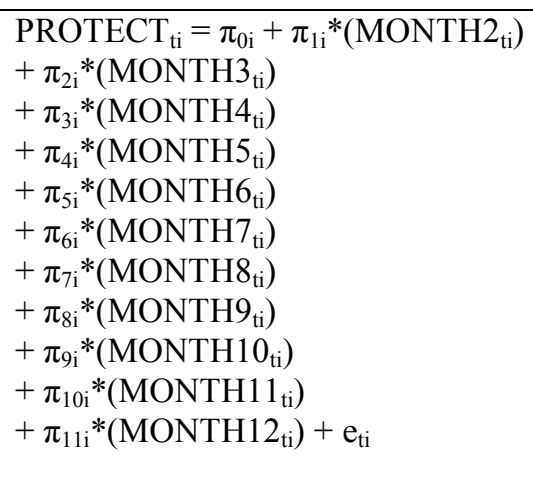 & $\begin{array}{l}\pi_{0 \mathrm{i}}=\beta_{00}+\mathrm{r}_{0 \mathrm{i}} \\
\pi_{1 \mathrm{i}}=\beta_{10} \\
\pi_{2 \mathrm{i}}=\beta_{20} \\
\pi_{3 \mathrm{i}}=\beta_{30} \\
\pi_{4 \mathrm{i}}=\beta_{40} \\
\pi_{5 \mathrm{i}}=\beta_{50} \\
\pi_{6 \mathrm{i}}=\beta_{60} \\
\pi_{7 \mathrm{i}}=\beta_{70} \\
\pi_{8 \mathrm{i}}=\beta_{80} \\
\pi_{9 \mathrm{i}}=\beta_{90} \\
\pi_{10 \mathrm{i}}=\beta_{100} \\
\pi_{11 \mathrm{i}}=\beta_{110}\end{array}$ & $\begin{array}{l}\text { PROTECT }_{\mathrm{ti}}=\beta_{00}+\beta_{10} * \mathrm{MONTH}_{\mathrm{ti}}+\beta_{20} * \mathrm{MONTH}_{\mathrm{ti}}+\beta_{30} * \mathrm{MONTH}_{\mathrm{ti}} \\
\quad+\beta_{40} * \mathrm{MONTH}_{\mathrm{ti}}+\beta_{50} * \mathrm{MONTH}_{\mathrm{ti}}+\beta_{60} * \mathrm{MONTH}_{\mathrm{ti}} \\
\quad+\beta_{70} * \mathrm{MONTH}_{\mathrm{ti}}+\beta_{80} * \mathrm{MONTH} 9_{\mathrm{ti}}+\beta_{90} * \mathrm{MONTH}_{\mathrm{ti}} \\
\quad+\beta_{100} * \mathrm{MONTH}_{1} 1_{\mathrm{ti}}+\beta_{110} * \mathrm{MONTH} 12_{\mathrm{ti}}+\mathrm{r}_{0 \mathrm{i}}+\mathrm{e}_{\mathrm{ti}}\end{array}$ \\
\hline Reconvictions & 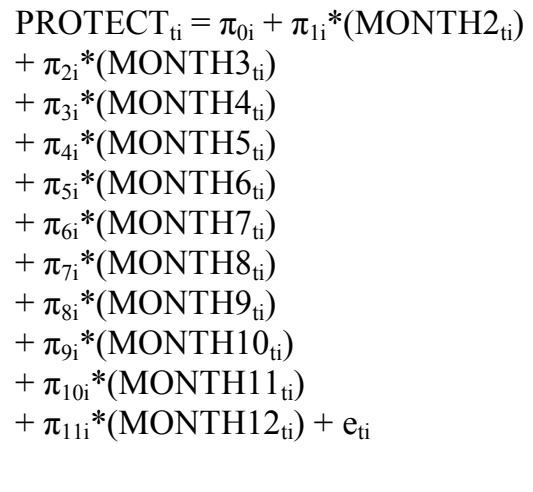 & $\begin{array}{c}\pi_{0 \mathrm{i}}=\beta_{00}+\beta_{01} *\left(\mathrm{FAIL}_{\mathrm{i}}\right)+\mathrm{r}_{0 \mathrm{i}} \\
\pi_{1 \mathrm{i}}=\beta_{10}+\beta_{11} *\left(\mathrm{FAIL}_{\mathrm{i}}\right) \\
\pi_{2 \mathrm{i}}=\beta_{20}+\beta_{21} *\left(\mathrm{FAIL}_{\mathrm{i}}\right) \\
\pi_{3 \mathrm{i}}=\beta_{30}+\beta_{31} *\left(\mathrm{FAIL}_{\mathrm{i}}\right) \\
\pi_{4 \mathrm{i}}=\beta_{40}+\beta_{41} *\left(\mathrm{FAIL}_{\mathrm{i}}\right) \\
\pi_{5 \mathrm{i}}=\beta_{50}+\beta_{51} *\left(\mathrm{FAIL}_{\mathrm{i}}\right) \\
\pi_{6 \mathrm{i}}=\beta_{60}+\beta_{61} *\left(\mathrm{FAIL}_{\mathrm{i}}\right) \\
\pi_{7 \mathrm{i}}=\beta_{70}+\beta_{71} *\left(\mathrm{FAIL}_{\mathrm{i}}\right) \\
\pi_{8 \mathrm{i}}=\beta_{80}+\beta_{81} *\left(\mathrm{FAIL}_{\mathrm{i}}\right) \\
\pi_{9 \mathrm{i}}=\beta_{90}+\beta_{91} *\left(\mathrm{FAIL}_{\mathrm{i}}\right) \\
\pi_{10 \mathrm{i}}=\beta_{100}+\beta_{101} *\left(\mathrm{FAIL}_{\mathrm{i}}\right) \\
\pi_{11 \mathrm{i}}=\beta_{110}+\beta_{111} *\left(\mathrm{FAIL}_{\mathrm{i}}\right)\end{array}$ & 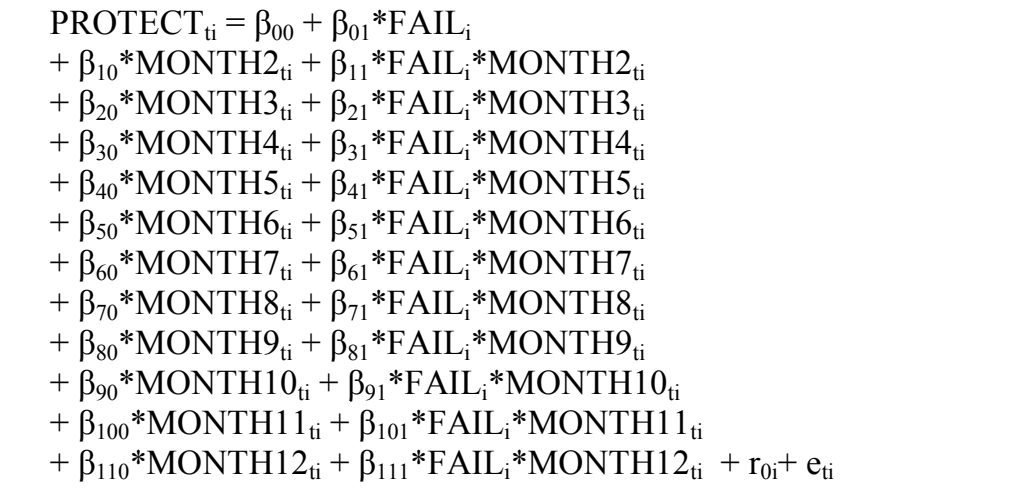 \\
\hline
\end{tabular}




\section{Criminal}

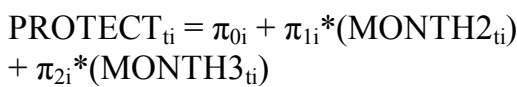

reconvictions

$+\pi_{3 \mathrm{i}} *\left(\mathrm{MONTH}_{\mathrm{ti}}\right)$
$+\pi_{4 \mathrm{i}} *\left(\mathrm{MONTH}_{\mathrm{ti}}\right)$
$+\pi_{5 \mathrm{i}} *\left(\right.$ MONTH6 $\left._{\mathrm{ti}}\right)$

$+\pi_{6 \mathrm{i}} *\left(\mathrm{MONTH} 7_{\mathrm{ti}}\right)$

$+\pi_{7 \mathrm{i}} *\left(\mathrm{MONTH} 8_{\mathrm{ti}}\right)$

$+\pi_{8 \mathrm{i}} *\left(\mathrm{MONTH} 9_{\mathrm{ti}}\right)$

$+\pi_{9 \mathrm{i}} *\left(\mathrm{MONTH} 10_{\mathrm{ti}}\right)$

$+\pi_{10 \mathrm{i}} *\left(\mathrm{MONTH} 11_{\mathrm{ti}}\right)$

$+\pi_{11 \mathrm{i}} *\left(\right.$ MONTH12 $\left.12_{\mathrm{ti}}\right)+\mathrm{e}_{\mathrm{ti}}$ $\pi_{0 \mathrm{i}}=\beta_{00}+\beta_{01} *\left(\mathrm{CFAIL}_{\mathrm{i}}\right)+\mathrm{r}_{0 \mathrm{i}}$ $\pi_{1 \mathrm{i}}=\beta_{10}+\beta_{11} *\left(\right.$ CFAIL $\left._{\mathrm{i}}\right)$

$\pi_{2 \mathrm{i}}=\beta_{20}+\beta_{21} *\left(\right.$ CFAIL $\left._{\mathrm{i}}\right)$

$\pi_{3 \mathrm{i}}=\beta_{30}+\beta_{31} *\left(\right.$ CFAIL $\left._{\mathrm{i}}\right)$

$\pi_{4 \mathrm{i}}=\beta_{40}+\beta_{41} *\left(\mathrm{CFAIL}_{\mathrm{i}}\right)$

$\pi_{5 \mathrm{i}}=\beta_{50}+\beta_{51} *\left(\right.$ CFAIL $\left._{\mathrm{i}}\right)$

$\pi_{6 \mathrm{i}}=\beta_{60}+\beta_{61} *\left(\mathrm{CFAIL}_{\mathrm{i}}\right)$

$\pi_{7 \mathrm{i}}=\beta_{70}+\beta_{71} *$ (CFAIL $\left._{\mathrm{i}}\right)$

$\pi_{8 \mathrm{i}}=\beta_{80}+\beta_{81} *\left(\mathrm{CFAIL}_{\mathrm{i}}\right)$

$\pi_{9 \mathrm{i}}=\beta_{90}+\beta_{91} *\left(\mathrm{CFAIL}_{\mathrm{i}}\right)$

$\pi_{10 \mathrm{i}}=\beta_{100}+\beta_{101} *\left(\mathrm{CFAIL}_{\mathrm{i}}\right)$
PROTECT $_{\mathrm{ti}}=\beta_{00}+\beta_{01} * \mathrm{CFAIL}_{\mathrm{i}}$

$+\beta_{10} * \mathrm{MONTH}_{\mathrm{ti}}+\beta_{11} * \mathrm{CFAIL}_{\mathrm{i}} * \mathrm{MONTH}_{\mathrm{ti}}$

$+\beta_{20} *$ MONTH $_{\mathrm{ti}}+\beta_{21} * \mathrm{CFAIL}_{\mathrm{i}} * \mathrm{MONTH}_{\mathrm{ti}}$

$+\beta_{30} * \mathrm{MONTH}_{\mathrm{ti}}+\beta_{31} * \mathrm{CFAIL}_{\mathrm{i}} * \mathrm{MONTH}_{\mathrm{ti}}$

$+\beta_{40} *$ MONTH $_{\mathrm{ti}}+\beta_{41} * \mathrm{CFAIL}_{\mathrm{i}} * \mathrm{MONTH}_{\mathrm{ti}}$

$+\beta_{50} *$ MONTH6 $_{\mathrm{ti}}+\beta_{51} *$ CFAIL $_{\mathrm{i}} *$ MONTH6 $_{\mathrm{ti}}$

$+\beta_{60} * \mathrm{MONTH}_{\mathrm{ti}}+\beta_{61} * \mathrm{CFAIL}_{\mathrm{i}} * \mathrm{MONTH}_{\mathrm{ti}}$

$+\beta_{70} *$ MONTH $_{\mathrm{ti}}+\beta_{71} * \mathrm{CFAIL}_{\mathrm{i}}^{*}$ MONTH $_{\mathrm{ti}}$

$+\beta_{80} *$ MONTH $_{\mathrm{ti}}+\beta_{81} * \mathrm{CFAIL}_{\mathrm{i}} * \mathrm{MONTH}_{\mathrm{ti}}$

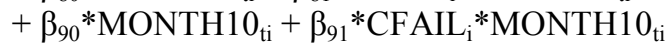

$+\beta_{100} *$ MONTH1 $11_{\mathrm{ti}}+\beta_{101} *$ CFAIL $_{\mathrm{i}} *$ MONTH $11_{\mathrm{ti}}$

Note. These models predict Protective Factors in all New Zealand parolees as a function of months prior to recidivism or censoring (at level-1) and all reconvictions or criminal reconvictions categorized into recidivists and non-recidivists (at level-2). The level-2 reconviction predictor is entered in uncentered form. 